\title{
Brain repair and general health improvement through human neurophysiology and repair physiology
}

\author{
Giselher Schalow* \\ Institute of Exercise Biology and Physiotherapy, Centre of Behavioural and Health Sciences, University of Tartu, 5 Jakobi Street, Tartu 51014, Estonia
}

\begin{abstract}
In this review of Coordination Dynamics Therapy (CDT) it will be shown that, using human neurophysiology, pathophysiology and repair physiology, the human Central Nervous System (CNS) can partly be repaired by movement-based learning. Since most body functions are controlled by the nervous system, these body functions can be improved through CDT. Since physical exercise also improves the immune system and the cardio-vascular performance, cancer growth can be inhibited. CDT therefore helps to reduce the occurrence of cancer, heart attack, stroke, hypertension and bladder incontinence mainly by improving the cardiovascular performance and CNS functioning. This general health improvement became possible by the development of human neurophysiology and basic medical research.
\end{abstract}

\section{Introduction}

Hodgkin and Huxley were able to clarify the excitation of animal nerve fiber membranes and the conduction of action potentials by using the squid giant axon which can have a diameter of up to $1 \mathrm{~mm}$ [1]. The Author used the special anatomy of the human caudal sacral nerve roots to build up human neurophysiology from the scratch to understand better the functioning of the human CNS and to repair it. When developing a new basic technique to record from single membrane channels in animals, one gets the Nobel prize in medicine even though this elegant technique has no direct consequences for medicine [2]. But when the Author developed a basic method to record from single human neurons under physiologic and pathologic conditions to develop repair treatment of the human brain and spinal cord, nearly nobody was even interested to look at the method. Qualified funding institutions of Germany and Switzerland did not want to support financially this medical research project and behaved as if the Author would be a little bit stupid even though having a very good education. The support of the former president of Germany, Richard von Weizsäcker, did not help to get funding. The Author was thrown out of the research society. The only country which supported this research project was the former East-Germany (GDR), even though the Author was at that time from West-Berlin. With the fall of the Berlin wall, the Author lost the infrastructure and became mainly a private researcher. Following 33 years of human research, the Author became able to partly repair the brain and spinal cord of children and adults, but the neurorehabilitation was and is still ignoring the repair progress. At the international conference in Belfast 2018 (IPBIS2018), the physicians ignored the poster of the Author. A rehabilitation physician from Switzerland even did not want to see the CNS repair of his own former patients Nefeli and Sophie. When it became possible to inhibit cancer growth and partly repair the brain of a patient being in the permanent coma through 6 years of CDT, a further important step was achieved towards general health improvement. This research project touches now forensic medicine. Is it allowed to let patients die or leaving them in the coma forever, if there is a chance to bring them back to life?
The important of this medical research is that it is not only possible to partly repair the human brain and spinal cord, but this treatment is based on human neurophysiology and can therefore developed further. It can be learned from CNS repair of patients to improve the health of mainly healthy humans. This review can be seen as a preliminary report of a nearly finished book: 'Neural Repair in Children, Parkinson, Aging, Hypertension, Coma and Cancer via Coordination Dynamics Therapy'. The Figures can be used in older books and the newer book to go deeper into the details of CNS functioning and repair [3-5]. The review is split into theory and praxis, even though the improvement of patients influenced the theory and the theory was used to adapt the therapy to the individual patient, because every injury is different. The Author thus believes in the unit of theory and praxis.

\section{Method}

The movement-based learning treatment 'Coordination Dynamics Therapy (CDT)' includes exercising on the special CDT device (Figure 1) to repair the impaired phase and frequency coordination of nervous system organization by means of imposing very coordinated arm, leg, and trunk movements in the lying, sitting and standing position and the training of the innate automatisms creeping (Figure 2), crawling (Figure 3A), up-righting, walking (Figure 3C,D) and running, so that other parts of the brain can take function over by plasticity. Further, rhythmic dynamic stereotyped movements, like jumping (Figure 34)

${ }^{*}$ Correspondence to: Giselher Schalow, Institute of Exercise Biology and Physiotherapy, Centre of Behavioural and Health Sciences, University of Tartu, 5 Jakobi Street, Tartu 51014, Estonia, E-mail: g_schalow@hotmail.com

Key words: human, morphometry, single-nerve fiber action potentials, surface emg, repair, coordination dynamics therapy, spinal cord injury, brain stem injury, brain injury, cerebral palsy, parkinson, tremor, ageing, general health improvement, urinary bladder continence

Received: March 02, 2019; Accepted: March 15, 2019; Published: March 18 2019 


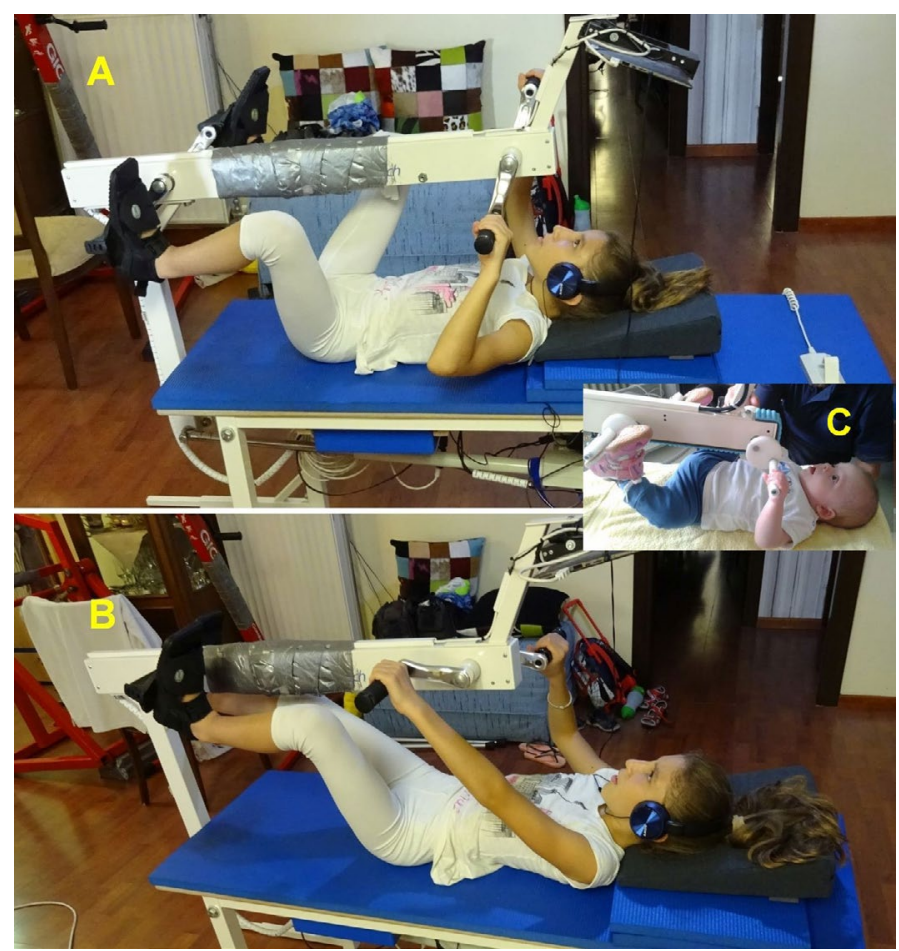

Figure 1. A, B. The 11-year-old Nefeli with an incomplete spinal cord injury (SCI) during exercising coordinated arm, leg and trunk movements in a more flexed (A) or extended (B) position to improve the coordinated firing of neurons and sub-neural networks. C. Device for babies. This special CDT device for measuring and therapy (int.pat.) is produced by the firm: Giger Engineering, Martin Giger dipl.Ing.ETH/SIA, Herrenweg 1, 4500 Solothurn, Switzerland, www.g-medicals.ch
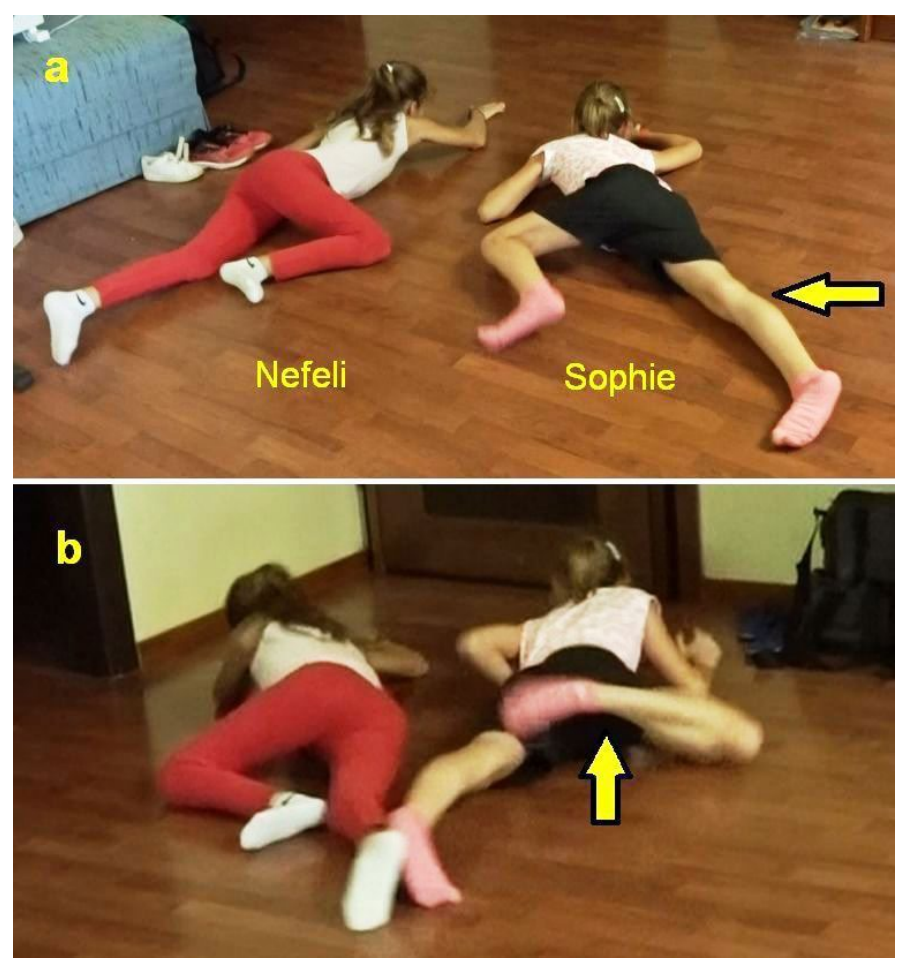

Figure 2. Sophie (cerebellum atrophy) during creeping in interpersonal coordination (antiphase) with Nefeli (SCI). Sophie is overstretching (a) and overswinging the legs (b) in comparison to Nefeli. She had not fully learned to control the inertia and centrifugal forces of leg movement. She cannot stop leg movement on time. The spinocerebellum (vermis) had not been repaired sufficiently so far and swinging on the springboard were exercised to entrain premotor spinal oscillators as an entrainment of CNS neuronal networks on the ensemble level. Old-learned integrative movements (learned automatisms), like climbing staircases or other movements like walking on knees (Figure 3B) or ride a bicycle (Figure 50), were also exercised. All movements were performed with an awareness of the importance of symmetry (Figure 3).

It is of importance to improve CNS functioning integrative (systemically), because the human CNS is an open system and if only a small sub-network is being entrained, the pathologic organization may escape the entrainment by shifting to another CNS neural network part.

\section{Results}

\section{Human neurophysiology}

Anatomy-Morphometry of nerves and nerve roots: The research to repair the human CNS starts from the scratch by analyzing the special micro-anatomy of the human lower sacral nerve roots to find a way to record at the single-neuron level action potentials [6,7]. Figure 4 shows the cross-section of a sacral S5 root and a ventral S4 root (Figure 4). It can be seen that the roots have no epineurium, which would shunt electrical activity as in peripheral nerves. Because of the Ascensus of the human spinal cord, the lower sacral nerve roots are long and thin (Figure 6A) and are suitable to record extracellular single-nerve fiber action potentials.

Even though it seems that the diameters of myelinated nerve fibers vary continuously in cross sections of nerves/roots, groups of nerve fibers can be recognized when classifying the mean nerve fiber diameters according to different myelin sheath thicknesses (Figure

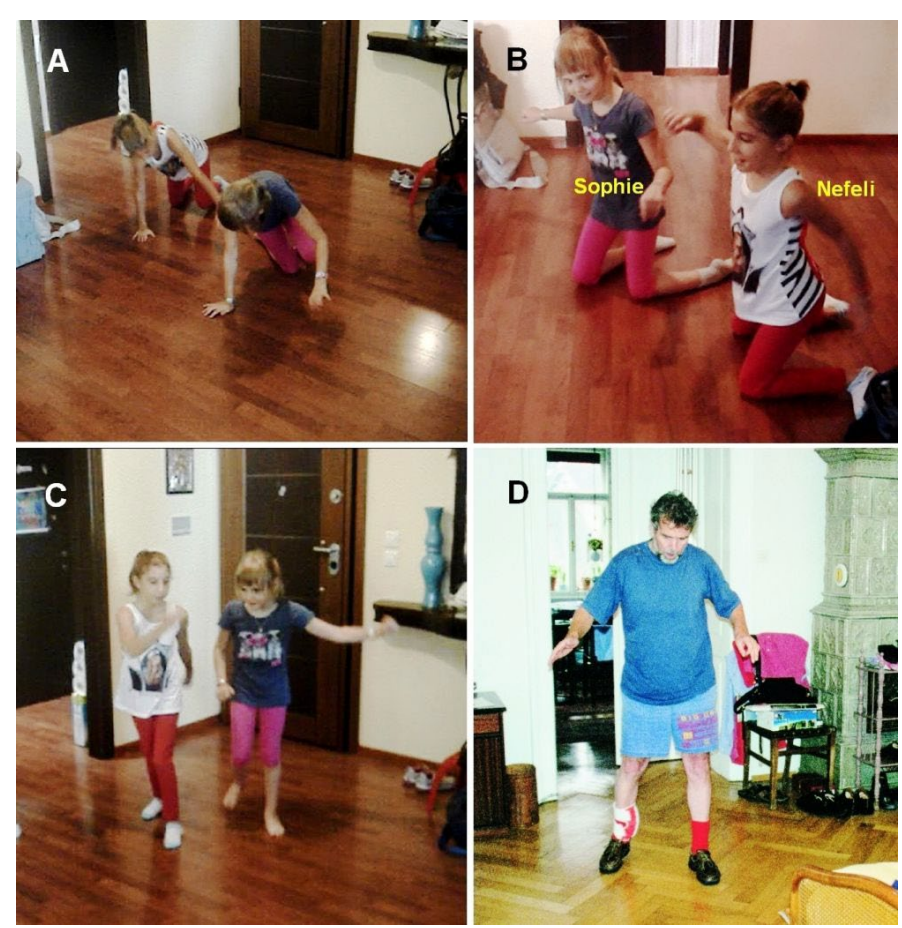

Figure 3. The SCI patient Nefeli and the cerebral palsy girl Sophie (cerebellum atrophy) are crawling (A), walking on knees (B) and walking (C) in interpersonal coordination. The walking in interpersonal coordination with Nefeli helped Sophie to walk longer distances before losing balance. Note that the walking pattern of Sophie with an atrophied cerebellum and ponds is completely different to the walking of an adult patient with a severe traumatic injury of cerebellum, pons and cerebrum (D) 

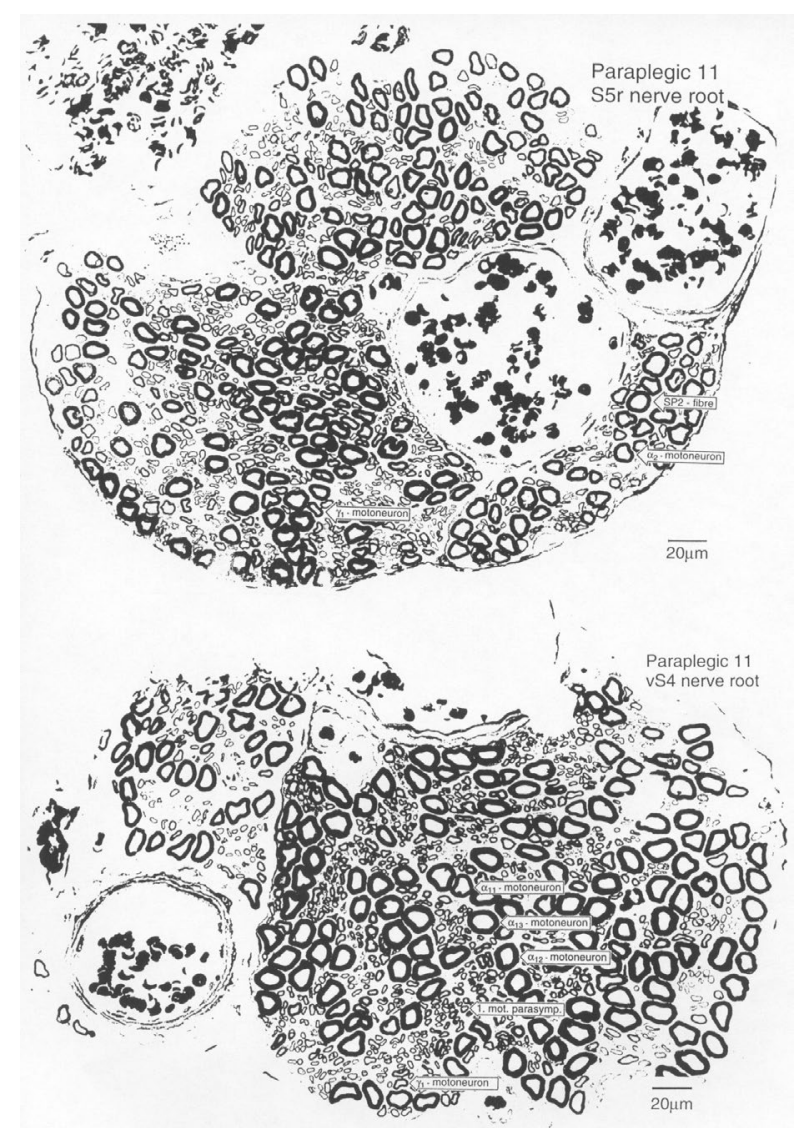

Figure 4. Light microscopic pictures of a cross section of the right nerve root S5 (A) and vS4 nerve root. Thionic acridine-orange staining of the myelin sheath. Paraplegic 11

5). In Figure 5Ba a large group of nerve fibers can be seen, which is identified with the classification scheme of below as the group of thick motoneuron axons which innervated fast fatigue muscle fibers. It can be seen further from the diameter spectra of Figure 5 that nerves or nerve roots have quite different nerve fiber compositions. For further morphometry see [3].

\section{Electrophysiology}

History: The question was now, is it really possible to record extracellular single-nerve fiber action potentials from human nerve roots? Since frog nerves have no epineurium, the Author tried to record from thin frog nerves whether action potentials can be recorded. It was possible, even with good quality (see below teaching electrophysiology, (Figure 71) [3, 4]. But still it was unclear whether it was possible to record single-nerve fiber action potentials extracellularly in humans, because the membrane resistances are different to those of frogs and rats. In rats the nerve fiber conduction velocities are up to $120 \mathrm{~m} / \mathrm{s}$. In humans the highest observed conduction velocities are approximately up to $70 \mathrm{~m} / \mathrm{s}$ (Figure 8 ). Further, the conduction velocity depends on the temperature. When the Author got the opportunity from a neurosurgeon to record in an operation, it turned out that it is possible to record from human nerve roots single-nerve fiber action potentials [8-10]. It was obvious for the Author that this will have consequences in medicine, especially for the repair of the human nervous system. It took him 20 to 30 years to prove it.

But to get specific functional information from the recordings of thin and long nerve roots, there should also not transported too many functions through the roots to identify functions and the activity should be low to be able to split the summed impulse traffic into patterns of single action potentials. In the dog, for example, the impulse patterns from the tale are mainly covering the urinary bladder functions [11-13]. But humans have no tail and through the sacral S4 and S5 roots mainly only urinary bladder, pelvic floor and sexual functions are leading. The coccygeal roots are mainly skin nerves.

Single-nerve fiber action potential recording method and a classification scheme for human peripheral nerve fibers: With the single-nerve fiber action potential recording method one can record at the neuron level simultaneously natural impulse patterns (Figure 6) running in and out of the spinal cord [8-13].

The impulse patterns of single-nerve fibers can be separated. But to identify from what nerve fibers the action potentials originate, a classification scheme for human nerve fibers is needed.

By measuring the conduction times and with the known electrode pair distance of $10 \mathrm{~mm}$, conduction velocity distribution histograms were constructed in which the myelinated nerve fiber groups larger than $4 \mu \mathrm{m}$ could be characterized by group conduction velocity values. After the recording, the roots were removed, fixated, stained and morphometry (Figure 5) was performed. Distributions of nerve fiber diameters for different myelin sheet thicknesses were constructed and nerve fiber diameter groups were characterized by the peak values of asymmetrical distributions. By correlating the peak values of the velocity distributions with those of the diameter distributions, obtained for the same root, a classification scheme was constructed for the human peripheral nervous system (Figure 7) [14]. A group of nerve fibers is thus classified by a group conduction velocity and a group nerve fiber diameter. This classification scheme (Figure 8) is the only existing one for the human nervous system. With this classification scheme it became possible to record natural impulse patterns simultaneously from identified single afferent and efferent nerve fibers and analyze self-organizing mechanisms of the human CNS under physiologic and pathologic conditions [3-5]. Since conduction velocities depend on the temperature, a calibration relation is needed. Such a calibration relation is for the lower sacral roots that the secondary muscle spindles afferent fibers conduct with the same velocity as the $\alpha_{2}$-motoneuron axons for different temperatures, marked in Figure 8 with red and in Figure 10 with a black double arrow. For the classification of human nerve fibers, it is further important that the nerve fibers taper only very little. As measured, if there is no splitting or branching of fibers, the tapering is $0.2 \%$ per $13 \mathrm{~cm}$ [15]. A $100 \mathrm{~cm}$ long fiber, for example of the pyramidal tract, would only reduce the diameter from $10 \mu \mathrm{m}$ to $9.8 \mu \mathrm{m}$.

Extension of the classification scheme: This human classification scheme only holds for myelinated nerve fiber groups larger than $4 \mu \mathrm{m}$, because for thinner fibers the action potential amplitude becomes too small and cannot be identified any more (Figure 71). In principle it is possible to extent the classification scheme to all nerve fibers. Morphometry can be performed for all fiber sizes, including the unmyelinated ones (Figure 8A). To get the group conduction velocities one would have to electrically stimulate the nerve roots or nerves and record compound action potentials [15-72]. The Author used the compound action potentials (comp) in Figure 10 (black bars) to have more safety concerning the velocity values of the nerve fiber groups.

A translation of the animal classification scheme to human is not possible, because the animal one is to inaccurate.

Skin, urinary bladder and muscle spindle afferent activity: When touching or pin-pricking sacral dermatomes, touch and pain afferent 

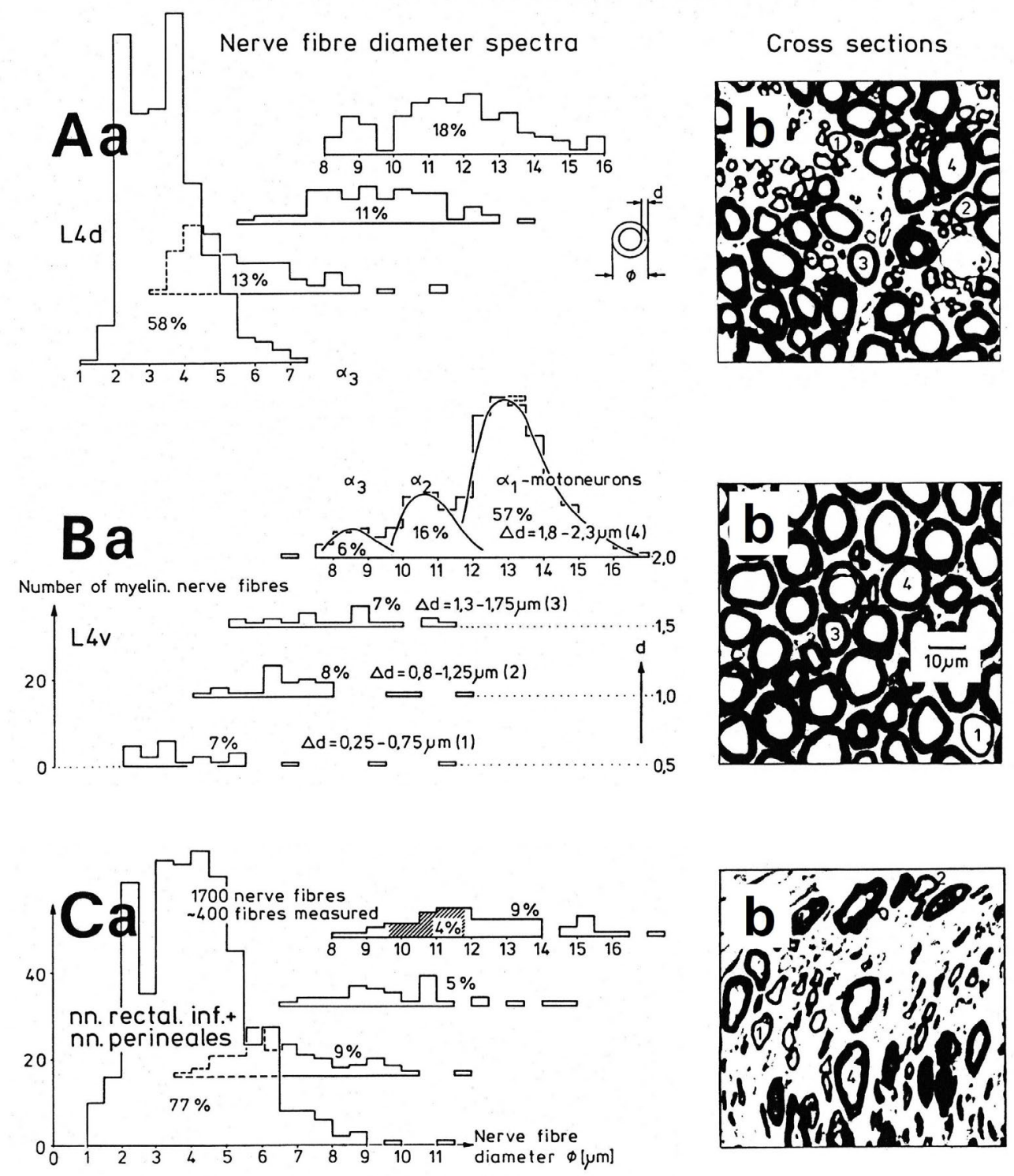

Figure 5. a. Nerve fibre diameter distribution histograms classified by 4 classes of myelin sheath thicknesses as indicated in Ba. \% indicates percentage of fibres in classes or subgroups. $\mathrm{b}$. Corresponding characteristic cross sections. A few fibres are numbered by their myelin sheath thickness range they belong to. Dimension scale for $\mathrm{A}, \mathrm{B}$, and $\mathrm{C}$ is drawn in $\mathrm{Bb}$. $8 \%$ shrinkage correction. For the definition of fibre diameter $\varnothing$ and myelin sheath thickness d see inset in Aa. - A. Nerve fibre diameter spectrum of a $4^{\text {th }}$ dorsal lumbar root of a 47 -year-old female human cadaver removed 2 to 5 hours after death, 660 fibres were measured. B. Spectra of a $4^{\text {th }}$ ventral lumbar root (same case as in A). 320 fibres were measured. In the myelin sheath thickness range $1.8 \leq \mathrm{d}<2.3 \mu \mathrm{m}$ the distribution curves of the $3 \alpha$-motoneuron classes are drawn into the histogram. C. Spectra of the nervi rectales inferiors and perineales. Note, the majority of fibres $(77 \%)$ has a very thin myelin sheath $(0.3 \leq \mathrm{d}<0.8 \mu \mathrm{m})$ with a relatively large amount of thick fibres. In the histogram of very thick myelin sheaths $(1.8 \leq \mathrm{d}<2.3 \mu \mathrm{m})$ the diameter range of $\alpha_{2}$-motoneurons, to which sphincter motoneurons belong, is crosshatched ( $4 \%$ of the fibre)

activity is running in sets of skin fibers into the spinal cord (Figure 9) $[16,17]$. The activity from the root recording (Figure 9A) can be split into the activity patterns of single touch (Figure $9 \mathrm{~Eb}$ ) and pain fibers (Figure 9F). These patterns are informing the neural networks of the CNS about the changes in the outside world. It can be seen from Figure 9D that the pain fibers have a small action potential amplitude.

When stimulating the urinary bladder or anal canal (rectum) by catheter pulling or retrograde bladder filling, bladder stretch, tension, fluid movement and mucosal afferent activity can be recorded [16,17]. Figure 10A shows single-fiber action potentials of a bladder afferent, a secondary muscle spindle afferent fiber and a $\gamma$-motoneuron. The corresponding frequency distribution histograms of afferent and efferent activity with the calibration relation (red) is shown in Figure 10B. Because in the lower sacral roots afferent and efferent fibers are mixed in dorsal and ventral roots, simultaneously the efferent activity leaving the sacral micturition center is simultaneously recorded (Figure 10C). The conduction velocity distribution histograms can be calibrated, and the different fibers identified (Figure 10).

Efferent (motor neuron) activity: When filling the urinary bladder during an operation retrogradely, afferent activity from the bladder receptors and sphincteric $\alpha_{2}$-motoneurons can be recorded (Figure 11). With little bladder filling, the internal bladder sphincter will secure continence. But with further filling the $\alpha_{2}$-motoneurons of the external bladder sphincter will be activated to further secure continence. 


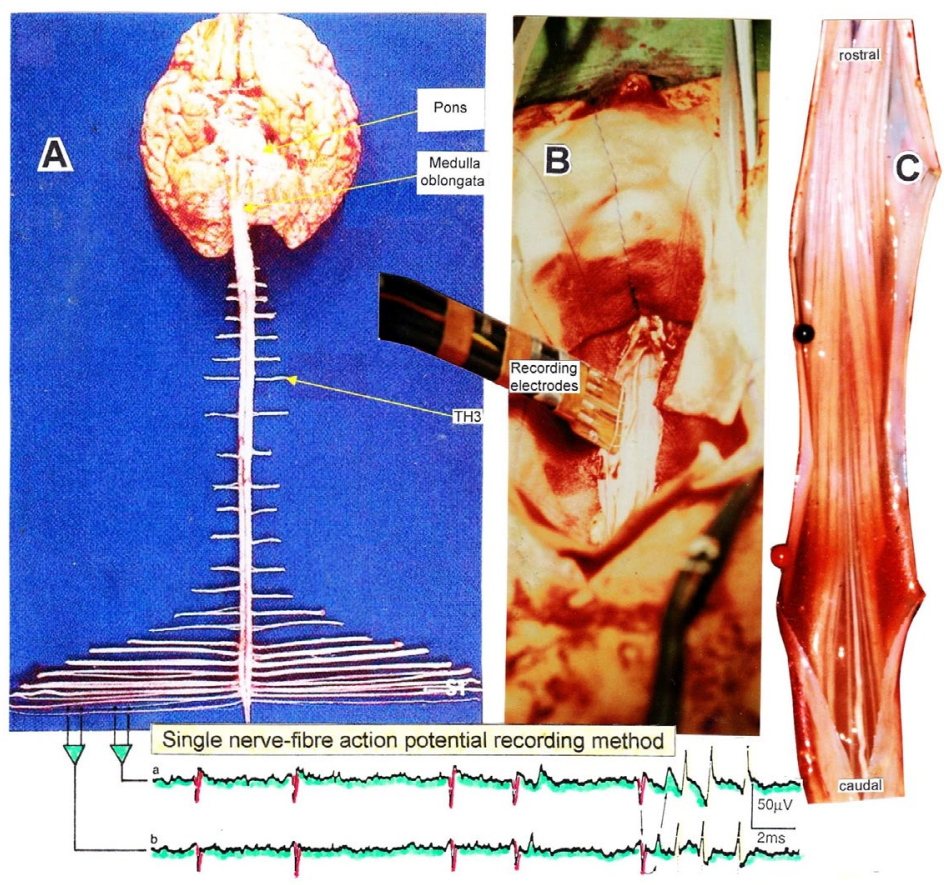

Figure 6. Layout of the recording of single-nerve fiber action potentials to measure the self-organization of neuronal networks of the human CNS under physiologic and pathophysiologic conditions. By recording with two pairs of wire electrodes (B) from sacral nerve roots (cauda equina, C), containing between 200 and 500 myelinated nerve fibers, records were obtained in which single-nerve fiber action potentials (APs) were identified from motoneuron axons (main action potential (AP) phase downwards) and afferents (main AP phase upwards)

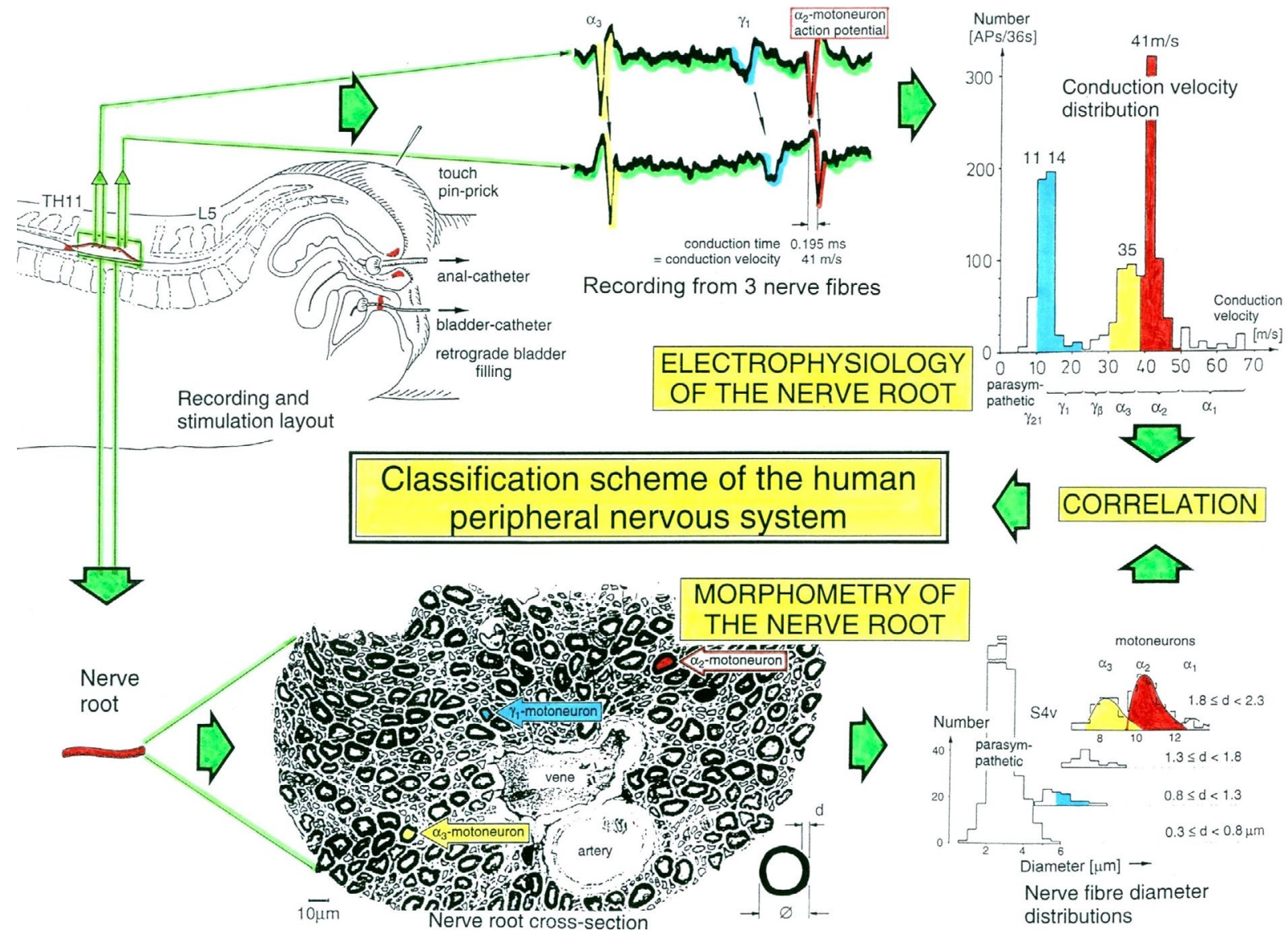

Figure 7. Development of a classification scheme for human peripheral nerve fibers. Conduction velocities (V) and nerve fiber diameters $(\varnothing)$ of afferent and efferent nerve fiber groups in normal humans and in patients with a traumatic spinal cord injury for 0.5 to 6 years 

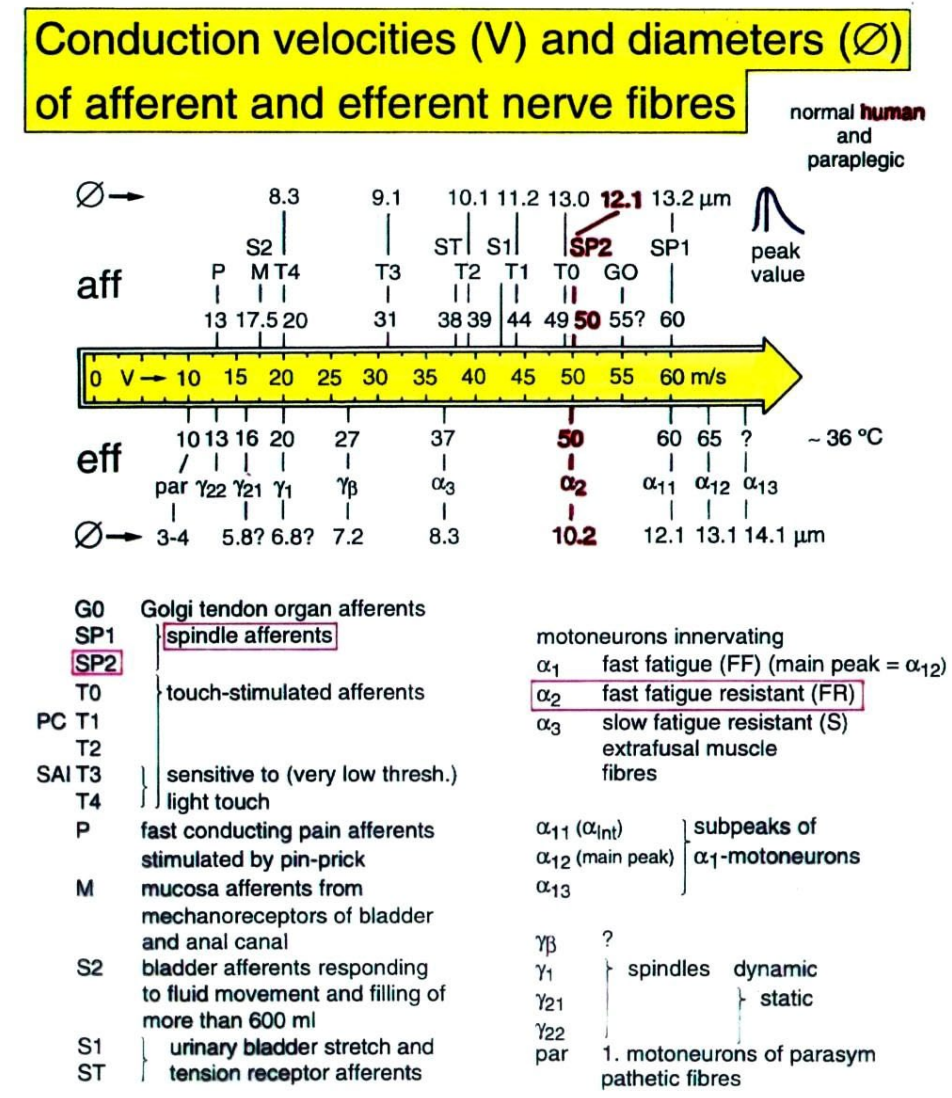

Figure 8. Classification scheme for human peripheral nerve fibers. Conduction velocities (V) and nerve fiber diameters $(\varnothing)$ of afferent and efferent nerve fiber groups in normal humans and in patients with a traumatic spinal cord lesion for 0.5 to 6 years. The splitting of the $\alpha_{1}$-motoneurons into the 3 subgroups, $\alpha_{11}, \alpha_{12}, \alpha_{13}$, has not yet been confirmed. This is the only existing classification scheme for human nerve fibers!
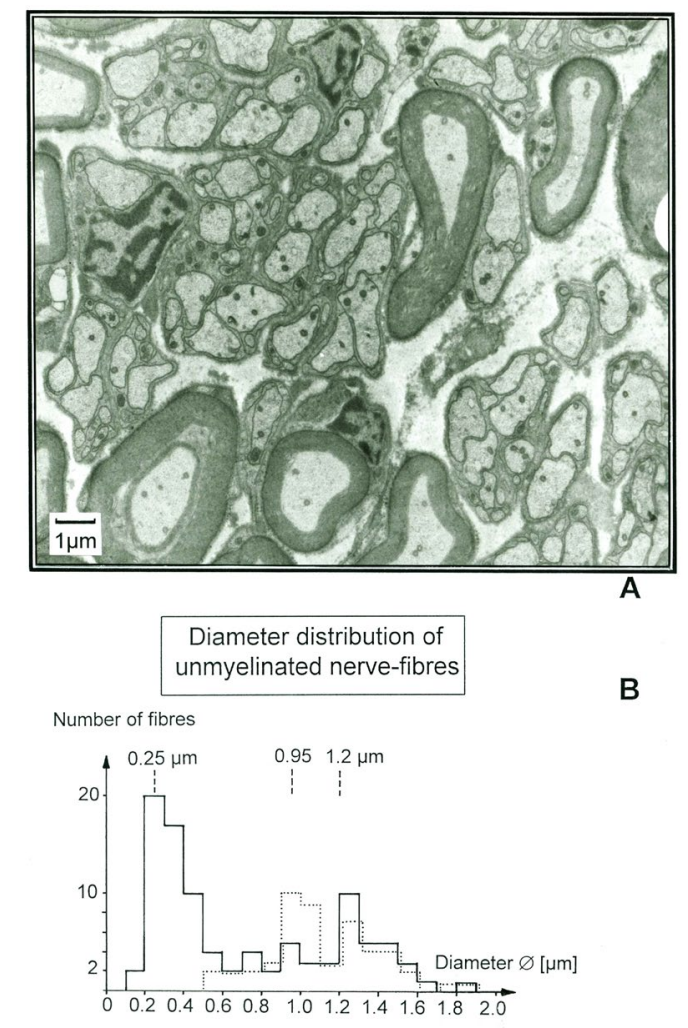

Figure 8A. A. Part of a cross-section of a ventral sacral S5 root of a 24 years old female paraplegic patient (para 5). Thin and thick unmyelinated nerve fibres can be seen besides myelinated nerve fibres. B. Nerve fiber diameter distributions of unmyelinated nerve fibres. For further see [3] 


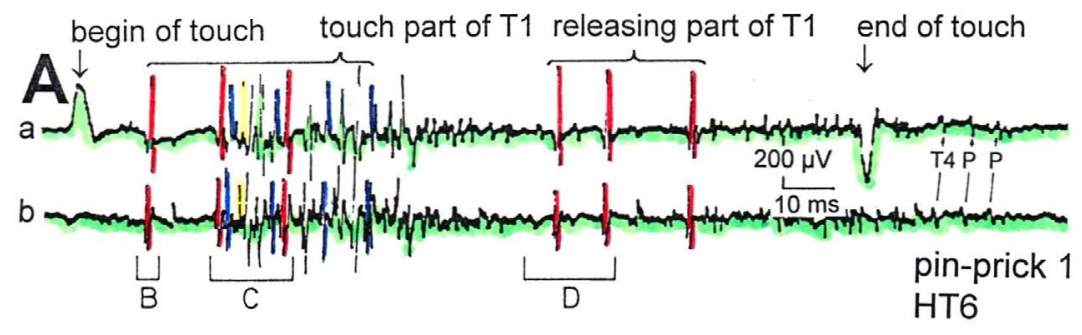

Touch (and pain) - stimulated afferent activity
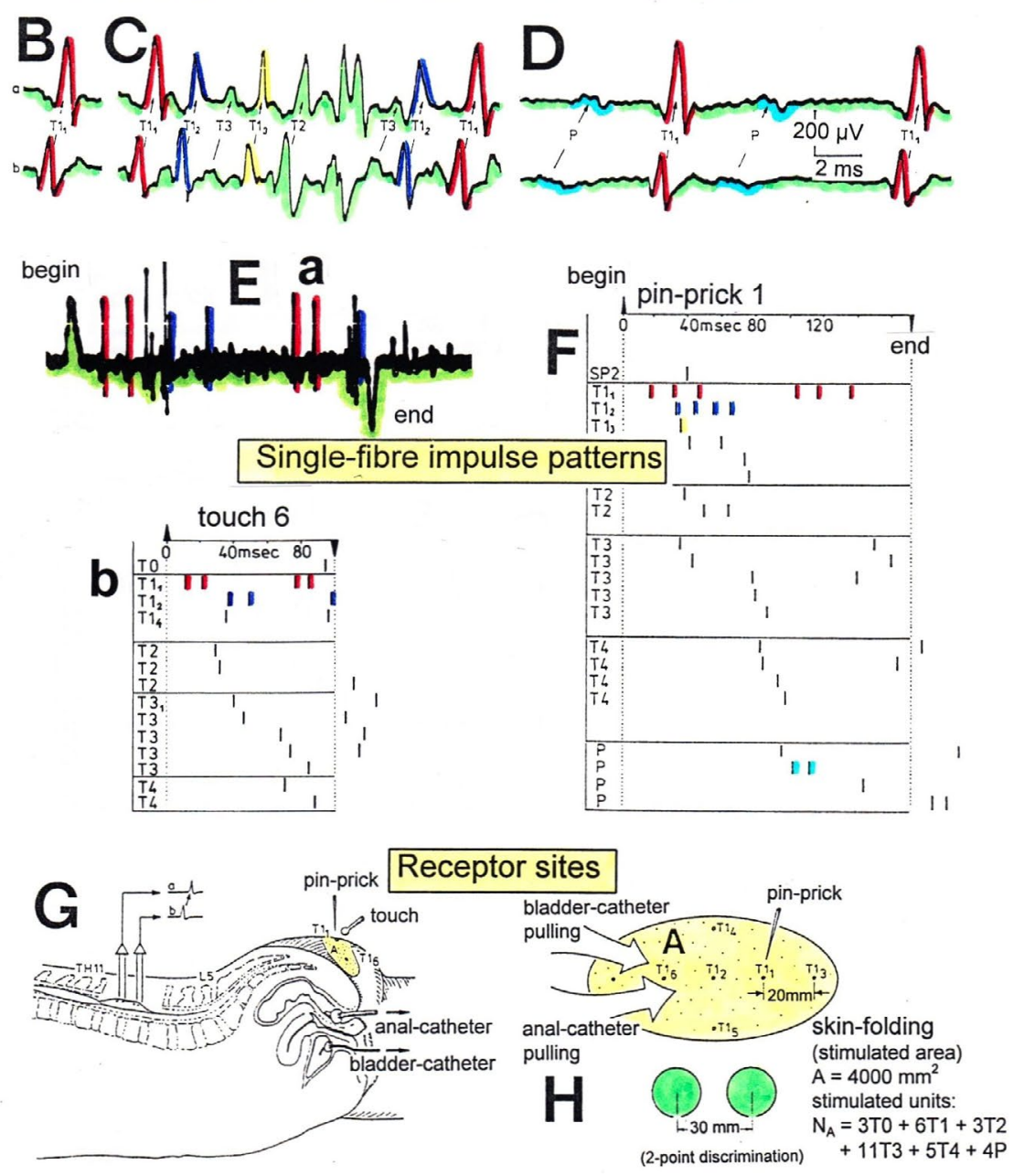

Figure 9. Touch (and pain)-stimulated afferent activity. Touch and pain activity stimulated by pin-prick (A) and touch (Ea) of S5 or Co dermatomes and recorded extracellularly from a dorsal coccygeal root (brain-dead human HT6). T1, T2, T3, T4, P = mark action potentials (APs) from single touch and pain fibers. Subscripts 1, 2, 3 mark single fibers

A. Whole sweep following pin-prick 1 shown at a slow time base. The large upward artifact on trace 'a' marks electronically the beginning of the pin-prick. The large downward artifact on trace 'a' marks the end of the pin-prick. Note that 2 intervals of high activity of large APs occur, one after the beginning of the pin-prick with 1 AP in front, and a second before the end of the pin-prick; potentials with large amplitude are followed by potentials of small amplitude. Time intervals B, C and D are shown in a time-expanded form in B, C and D.

B, C, D. Time expanded sweep pieces of A. Identified APs are indicated. Note that the APs from the T1, touch unit can be safely identified by the waveforms in B, C, D.

$\mathrm{Eb}, \mathrm{F}$. AP occurrence patterns of single touch and pain fibers following short touch 6 and pin-prick 1. No pain afferents are stimulated upon touch 6 . Upon pin-prick 1, the single-fiber AP activity of the different touch and pain groups is identified by the AP waveforms on traces ' $a$ ' and ' $b$ ', and by the conduction times. The single touch afferents of the T1 group are marked with subscripts. One active secondary muscle spindle afferent fiber (SP2) could always be identified in F. Note that for pin-prick 1, touch and pain afferents are stimulated whereas for touch 6 only touch afferents.

G. Recording and stimulation arrangement for simultaneous recording of several single touch and pain units. $\mathrm{A}=$ area stimulated by skin folding, drawn in $\mathrm{H}$ in more detail. $\mathrm{T} 1_{1}$, $\mathrm{T} 1_{6}=$ suggested touch points of the $\mathrm{T} 1_{1}$ and $\mathrm{T} 1_{6}$-units.

$\mathrm{H}$. Drawing of the very approximate skin area stimulated by skin folding. $\mathrm{T} 1_{1-6}=$ suggested focal $\mathrm{T} 1$ touch points. Two-point discrimination indicated for the sake of comparison. $\mathrm{N}_{\mathrm{A}}=$ number of stimulated units in the dorsal coccygeal root. Skin tractions evoked by anal and bladder-catheter pulling are indicated by the large open arrows. 
Because the sphincteric $\alpha_{2}$-motoneurons are continuously more and more activated, the firing modes of $\alpha_{2}$-motoneurons can be studied.

Figure $12 \mathrm{D}$ shows the firing modes for increasing activation due to retrograde bladder filling. For low activation (little bladder filling) the $\alpha_{2}$-motoneuron $\mathrm{O} 1$ of the external bladder sphincter (striated muscle) fires occasionally. With further activation the motoneuron fires transient oscillatory and then continuously oscillatory $[18,19]$. These are the firing modes of human $\alpha_{2}$-motoneurons. The oscillation frequency varied between 7 and $9 \mathrm{~Hz}$. Before continuing with the firing modes of the different kinds of human motoneurons, the recording situation is given to have further insight into the organization of the human CNS.
In the measuring situation of Figure 12 it is recorded from the $\alpha_{2}$-motoneurons $\mathrm{O} 1$ and $\mathrm{O} 2$. $\mathrm{O} 1$ innervates the external urinary sphincter and $\mathrm{O} 2$ the external anal sphincter or the pelvic floor and is not involved in the continence function of the urinary bladder. The oscillatory firing patterns are given in Figure 12A. Simultaneously the activity is recorded from the bladder stretch (S1), tension (ST) and fluid movement (S2) receptors and 3 secondary muscle spindle afferents (SP2(1-3)). With retrograde bladder filling, the firing of the stretch and tension receptors is increasing (Figure 12E). The receptors for fluid movement (S2) fire only little. The S2 receptors increase their firing strongly for very high bladder filling. Probably fluid is leaking from the bladder. For low bladder filling the sphincteric $a_{2}$-motoneuron of the bladder (striated muscle, somatic nervous system) is only little

\section{Conduction velocities and nerve fibre groups
(no stimulation)}

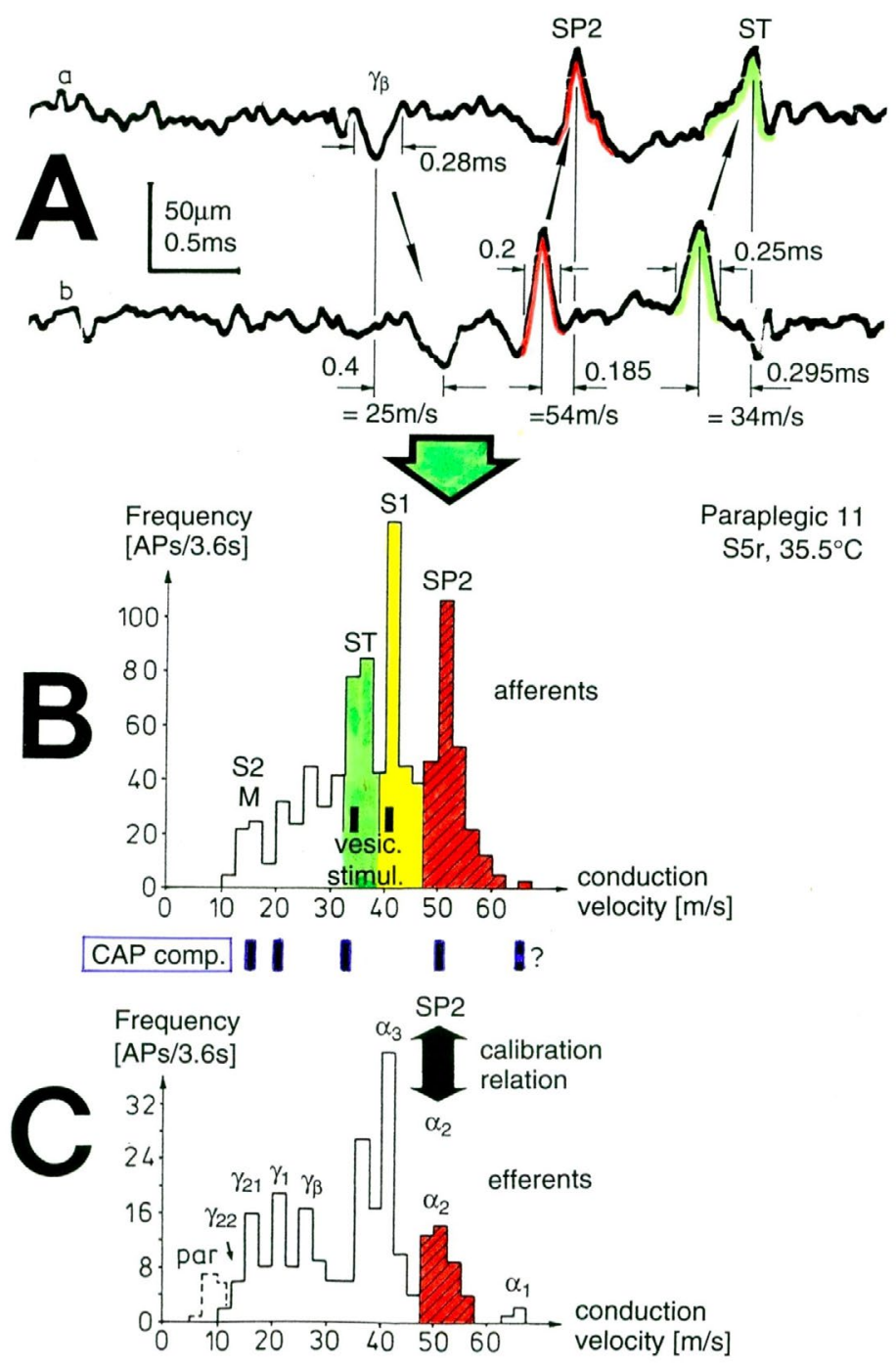

Figure 10. A. Sweep piece of recording; conduction times and corresponding conduction velocities are indicated. Root temperature at recording electrodes, $35.5^{\circ} \mathrm{C} . \mathrm{B}, \mathrm{C} . \mathrm{Conduction}$ velocity distributions of afferents (B) and efferents (C) obtained for a time interval of $3.6 \mathrm{~s}$ with no additional stimulation. SP2 $=$ secondary muscle spindle afferents, S1 $=$ stretch receptor afferents of bladder, $\mathrm{ST}=$ tension receptor afferents, $\mathrm{M}=$ mucosal afferents, $\mathrm{S} 2=$ afferents responding to fluid movement; $\alpha_{1}=\alpha_{1}$-motoneurons $(\mathrm{FF}), \alpha_{2}=\alpha_{2}$-motoneurons $(\mathrm{FR}), \alpha_{3}=\alpha_{3}$ motoneurons (S), $\gamma_{B}=\gamma_{B}$-motoneurons, $\gamma_{1}=\gamma_{1}$-fusimotors (dynamic), $\gamma_{21}=\gamma_{21}$-fusimotors (static), $\gamma_{22}=\gamma_{22}$-fusimotors (static), par $=$ preganglionic parasympathetic motoneurons. CAP comp. $=$ group conduction velocities obtained from the components of compound action potentials (CAPs). Vesic. stimul. = group conduction velocities of bladder afferents obtained upon electrical intravesical stimulation. Calibration relation indicates the same peak group conduction velocity of secondary spindle afferents and $\alpha_{2}$-motoneurons 


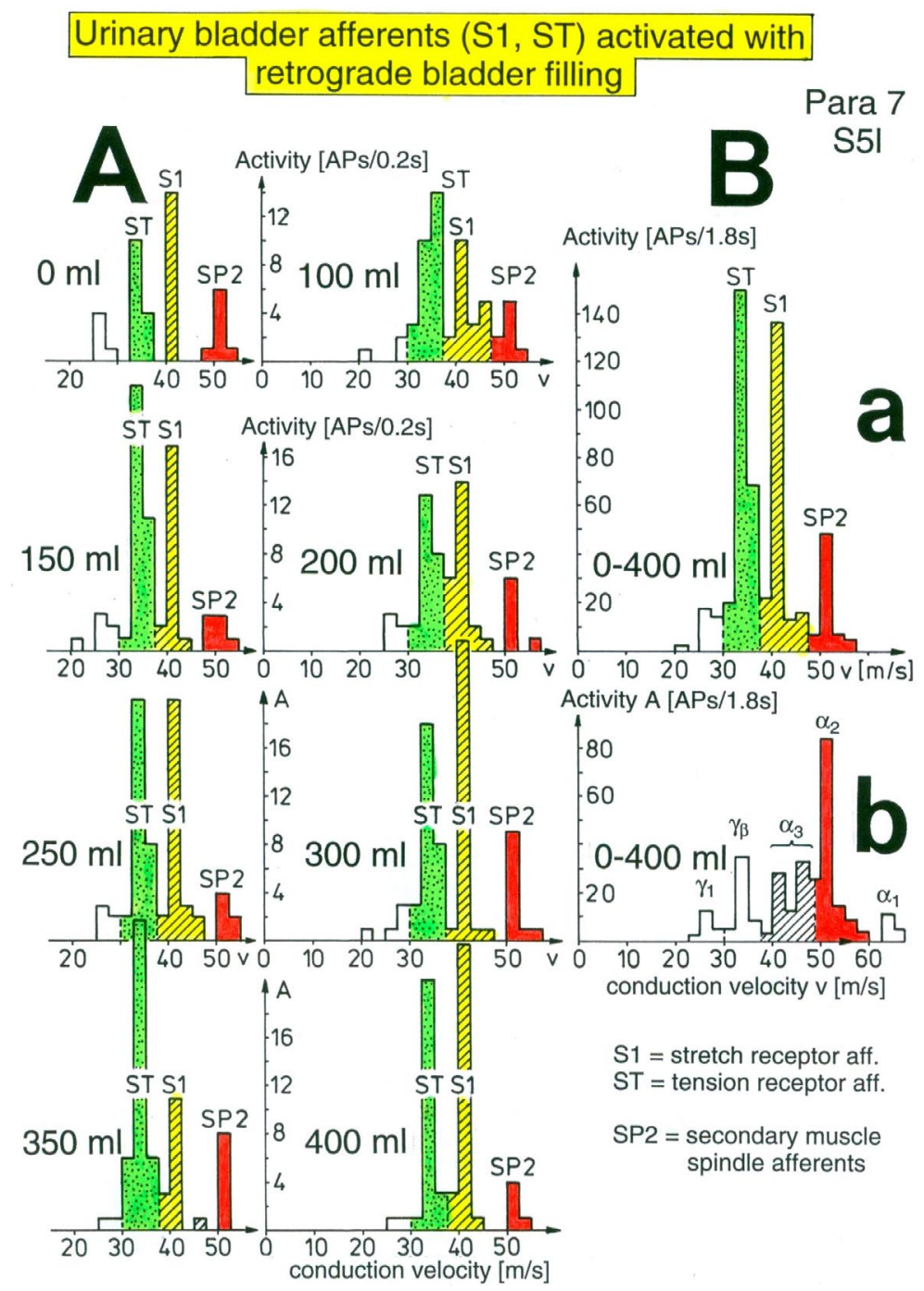

Figure 11. A. Conduction velocity distribution histograms for stretch (S1) and tension receptor afferents (ST) and secondary spindle afferents (SP2) for different retrograde filling stages of the urinary bladder. B. Summed histograms for afferents (a) and efferents (b) are plotted. $\alpha_{1}, \alpha_{2}, \gamma_{\beta}, \gamma_{1}$ represent velocity distributions of $\alpha_{1}, \alpha_{2}, \gamma_{\beta}$ and $\gamma_{1}$-motoneurons. Para 7, left nerve root S5

activated (Figure 12). The continence is secured by the inner bladder sphincter (smooth muscle, sympathetic division). This is the storage phase of the bladder. When the bladder is very full, the external bladder sphincter is securing additionally the continence. When the bladder is nearly over-filled, the external bladder sphincter becomes inhibited not to damage the bladder. The overflow mechanism takes place and the receptors for fluid movement (S2) are strongly activated. Also, for low bladder filling the external bladder sphincter is activated to secure continence when the patient is cuffing, because the inner sphincter is too slow (smooth muscle).

As demonstrated, the functioning of the urinary bladder can be understood at the single-neuron level. Only the parasympathetic nervous system seems to be missing. The autonomic nervous system innervates the sacral micturition center and the urinary bladder directly. The sympathetic division innervated the detrusor and the parasympathetic division the muscles spindles of the striated muscles. It is discussed whether the sacral parasympathetic division is really sympathetic [20]. Since morphometry and electrophysiology are missing in this report, the conclusion is not safe. Below the autonomic innervation of the muscle spindles of the external bladder sphincter is analyzed.
The most important finding with this human neurophysiology is that the neural networks of the human brain (generally CNS) are organizing themselves by phase and frequency coordination which is analyzed below [21-23]. In Figure $12 \mathrm{~B}$ it can be seen that the motoneurons, the secondary muscle spindle afferents and a bladder afferent fiber fired with respect to phase and frequency coordination, indicated by arrows and dotted lines.

Now we are coming to the firing patterns of motoneurons. In Figure 12D it is shown that an $\alpha_{2}$-motoneuron fired occasional, transient oscillatory and continuously oscillatory. As shown in Figure 13, the three kinds motoneurons are integrated in their own neural networks, have their own oscillation frequency and innervate their own muscle fiber type (Figure 13) $[18,19]$. The $\alpha_{1}$-motoneurons fire with $10 \mathrm{~Hz}$ or more with one action potential per impulse train and innervate fast fatigable muscle fibers (white). The oscillatory property is low. The $\alpha_{2}$-motoneurons fire with 6 to $9 \mathrm{~Hz}$ with 2 to 4 action potentials per impulse train and innervate fast fatigable resistant muscle fibers. The oscillatory property is high. As will be shown below, their oscillatory firing will give rise to tremor in patients with Parkinson's disease. The $\alpha_{3}$-motoneurons fire with approximately $1 \mathrm{~Hz}$, have up to 


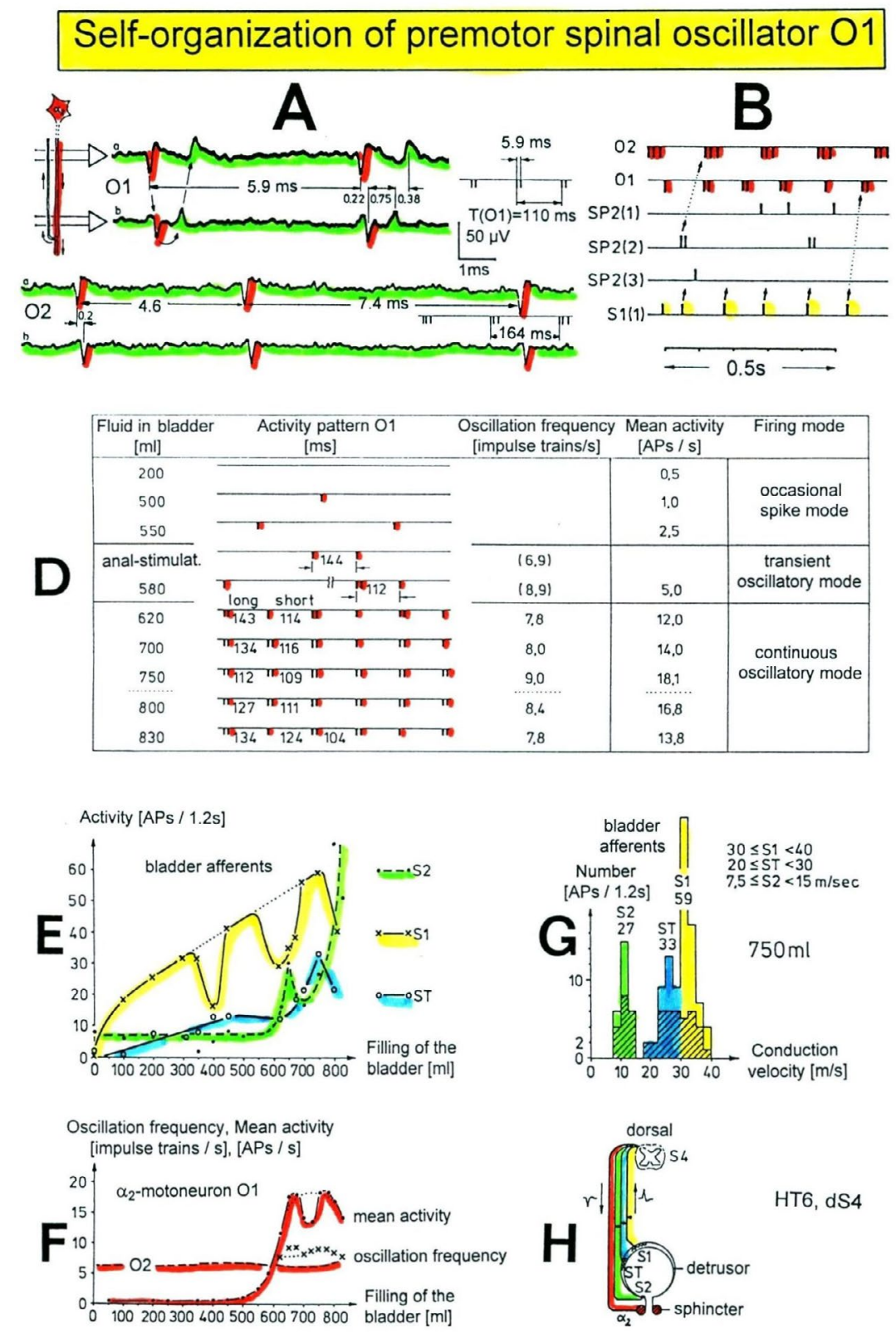

Figure 12. Self-organization of premotor spinal aa 2 -oscillator $\mathrm{O}_{1}$, which innervates the external urinary bladder sphincter (skeletal muscle). Brain-dead human HT6; recording from a dorsal S4 nerve root

A. Recordings from $\alpha_{2}$-motoneurons $\mathrm{O}_{1}$ and $\mathrm{O}_{2}$, firing in the oscillatory mode with impulse trains of 2 (upper recording) and 3 (lower recording) action potentials (APs). The durations of the oscillation periods were $110\left(\mathrm{O}_{1}\right)$ and $164 \mathrm{~ms}\left(\mathrm{O}_{2}\right)$. The interspike intervals of the impulse trains were $5.9 \mathrm{~ms}\left(\mathrm{O}_{1}\right)$ and 4.6 and $7.4 \mathrm{~ms}\left(\mathrm{O}_{2}\right)$. Motoneuron $\mathrm{O}_{1}$ conducted at $36 \mathrm{~m} / \mathrm{s}$; its recurrent fiber conducted at $21 \mathrm{~m} / \mathrm{s}$. The measurement layout is shown schematically. The inserts show the oscillatory firing modes; they have not been drawn to scale.

B. Impulse patterns of oscillatory firing $\alpha_{2}$-motoneuron $\mathrm{O}_{2}$ innervating the external anal sphincter, in relation to the muscle spindle afferent activity SP2(1 to 3 ), activated by the stretch of the anal sphincter by the anal catheter, and impulse patterns of oscillatory firing $\alpha \mathrm{a}_{2}$-motoneuron $\mathrm{O} 1$ innervating the external urethral sphincter, in relation to the stretch receptor afferent activity (S1(1)) of the urinary bladder, activated by $750 \mathrm{ml}$ bladder filling. Phase relations between APs of SP2(2) and $\mathrm{O}_{2}$ and between APs of S1(1) and $\mathrm{O}_{1}$ are indicated by the small arrows. D. The firing in the occasional spike mode, the transient and the constant oscillatory firing mode of $\alpha a_{2}$-motoneuron $\mathrm{O}_{1}$ in response to filling of the bladder. In the 'activity pattern' column changing durations of oscillation periods are given. The oscillation frequencies in the brackets give the frequencies at the moment of oscillation for the transient oscillatory mode. Downward deflections are schematized APs. Interspike intervals of the close APs $\approx$ » $6.0 \mathrm{~ms}(\mathrm{~A})$.

E. Activity levels of stretch (S1) and tension (ST) and flow receptor afferents (S2) (E) and of sphincter $\alpha_{2}{ }_{2}$-motoneuron $\mathrm{O}_{1}(\mathrm{~F})$ in response to retrograde filling of the bladder. The activity values of the S1, ST and S2 afferents are taken from histograms like the one in G. Filling of the bladder was stopped once between 600 and $650 \mathrm{ml}$.

F. The small dotted lines represent mean activity (APs/s) and oscillation frequency (impulse trains/s) of $\alpha a_{2}$-motoneuron $\mathrm{O}_{1}$ if bladder filling were not stopped in between. Note that the mean activity increases continuously with the filling of the bladder from 550 to $650 \mathrm{ml}$, even though motoneuron $\mathrm{O}_{1}$ started to fire in the oscillatory mode from $620 \mathrm{ml}$ on (D). Note further that the oscillatory firing motoneuron $\mathrm{O}_{2}$ (frequency of firing with impulse trains is shown) is nearly not affected by the filling of the bladder and by the start of the oscillatory firing of motoneuron $\mathrm{O}_{1}$. G. Conduction velocity frequency distribution histogram of stretch, tension and flow receptor afferent activity at $750 \mathrm{ml}$. The activities of afferents S1, ST and S2 are quantified by counting the afferent conduction velocities under the peaks (open plus hatched part), with the conduction velocity limits given in the insert. The counts (27, 33, 59) are given below the peak labeled $\mathrm{S} 1, \mathrm{ST}$ and $\mathrm{S} 2$ and plotted into E for the afferent activity at $750 \mathrm{ml}$.

$\mathrm{H}$. Schematic drawing of the anatomical arrangement of the afferents and the motoneuron $\mathrm{O}_{1}$ 


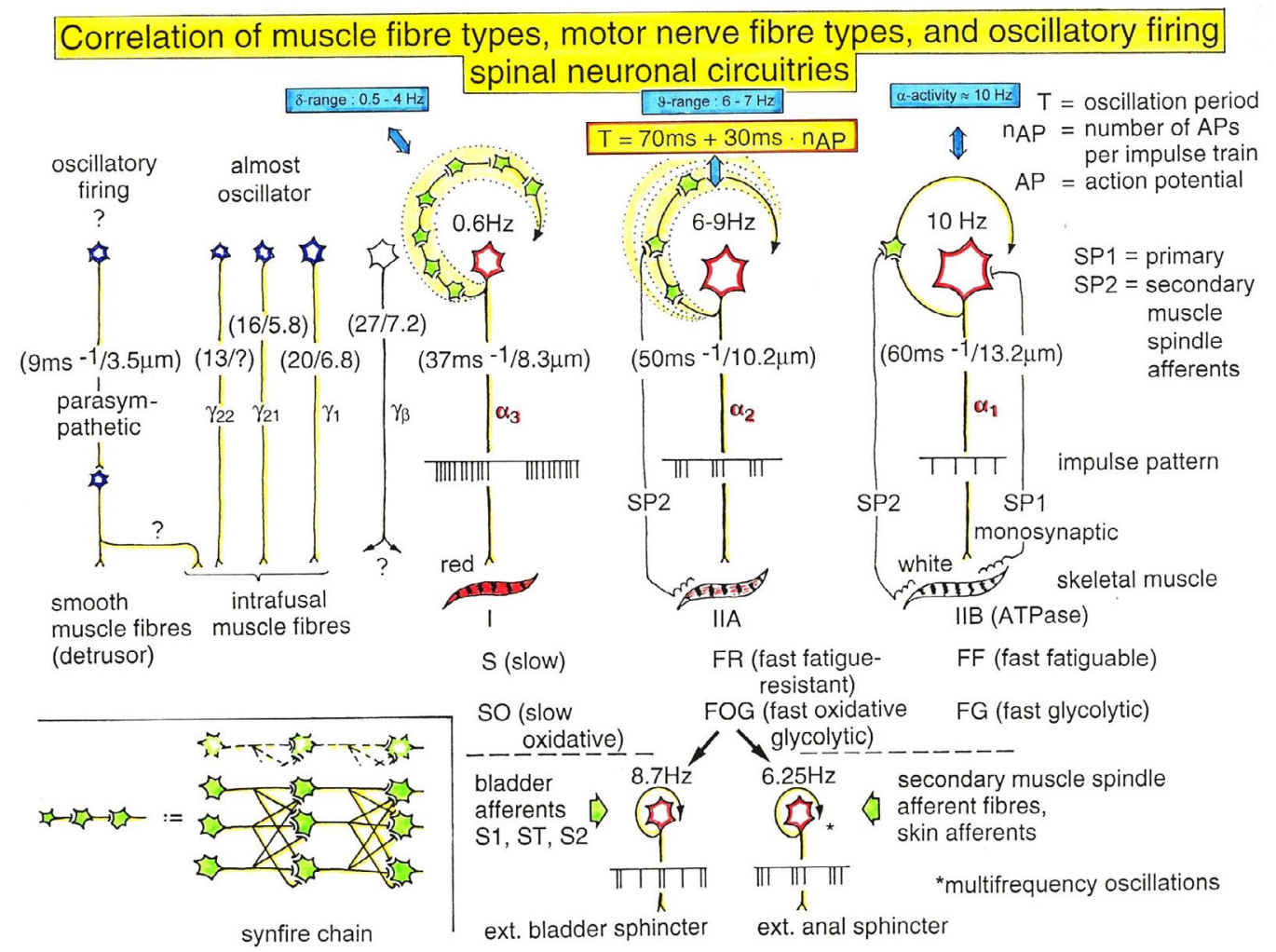

Figure 13. Correlation of muscle fiber types, motor nerve fiber types, and oscillatory firing spinal neuronal networks, based on histochemical, morphological and physiological properties. This figure provides a simplified correlation between muscle fiber, motoneuron and sacral oscillator types. No additional subtypes have been included. $\alpha=$ motoneuron, $\gamma_{1}, \gamma_{2}=$ dynamic and static fusimotors, parasympathetic $=$ parasympathetic preganglionic motoneuron. S1, ST, S2 $=$ stretch, tension and flow receptor afferents

50 action potentials per impulse train and innervate slow (red) muscle fibers. Their oscillatory property is probably high.

Phase and frequency coordination-an organization principle of the human brain: The most important finding in this research project is the 'phase and frequency coordination' among neuron firings [21-24]. If one really wants to understand the functioning of the human brain, then one has to use the knowledge of the coordinated firing among neurons and may be find other organization principles. Clinically this 'phase and frequency coordination' is of very high importance, because with every injury, malformation or degeneration, this coordination among neuron firing becomes impaired with the consequence of loss of CNS functions and has to be repaired to regain important functions back by therapy like urinary bladder functions and consciousness in coma patients (see below). When exercising on a special CDT device (Figure 1), this coordinated firing can be improved in the deepness of complexity of CNS organization. The difficult coordination's between arm, leg and trunk movements between pace and trot gait, imposed by the device, seem to re-unite the firing of the many neurons in extreme severe injuries so that even patients in the permanent coma have a chance to come back to life again (see below).

In Figure 12B the 'phase and frequency coordination' was shown schematically among neurons responsible for physiologic urinary bladder functioning. But this coordinated firing of neurons is the basis for all neural network functions of the brain. The frequency coordination will be shown among secondary muscle spindle afferent fibers and an $\alpha$ and $\gamma$-motoneuron and in Figure 14A, in another recording, bladder afferents will be included.
In Figure 14, considerations concerning the relative frequency coordination is shown for different secondary spindle afferent fibers and $\gamma g$-motoneurons of the same root. Sweep pieces of the original recordings are shown in ' $G$ '. A through $\mathrm{F}$ shows the interspike interval distributions of spindle afferents and $\gamma$ g-motoneurons. It can be seen from the overlapping of the oscillator frequency $\mathrm{T}(\mathrm{O} 2)$ and $\mathrm{T}(\mathrm{O} 2) / 2$ distribution ranges and the interspike interval distributions of the afferents that, from the viewpoint of frequency coordination, fiber SP2(1) contributed strongly to the drive of oscillator O2, whereas there was a weaker contribution from other afferents, i.e. less overlapping between the distributions of the afferents and the range of the basic frequency or the first harmonic of the oscillator. Also, $\gamma g$-motoneurons showed only little frequency correlation at that time period (Figure 14).

Figure 14A shows the interspike interval distributions of more afferents (including the afferents for bladder filling; stretch receptor afferents S1(1), S1(2)) of another root, together with the oscillation period range (and the half of it) of a second $\alpha \alpha_{2}$-oscillator (O1). By comparing the oscillation periods (and their halves) and their ranges with the interspike interval distributions of the afferents, it can be suggested which afferents made a contribution to the drive of what oscillator at that time interval. For example, the S1(1) urinary bladder stretch afferent fibre activity contributed to the drive of oscillator O1 (activating the external bladder sphincter) because its interspike interval distribution overlaps strongly with the range of the oscillation periods of O1. But the S1(1) distribution does not overlap with the range of the oscillation periods of oscillator $\mathrm{O} 2$, or with their halves or quarters. The S1(1) afferent fibre will therefore not have made a substantial contribution to the drive of oscillator $\mathrm{O} 2$. On the other hand, 
the secondary muscle spindle afferent fibre SP2(12) activated oscillator $\mathrm{O} 2$, innervating the external anal sphincter, since its interspike interval distribution overlaps with the range of $\mathrm{O} 2$ oscillation periods. But the secondary muscle spindle afferent fibre SP2(12) did not activate oscillator $\mathrm{O} 1$, as its interspike interval distribution does not overlap with its oscillation period range or the half of it.

The fact that neurons fire in a relatively coordinated way of up to a few milliseconds is used for re-organizing the injured CNS by relearning phase and frequency coordination between neuron firings when exercising movements coordinated with an exactness of up to a few milliseconds, using a special coordination dynamic therapy device, i.e. by instrumented supervised phase and frequency re-learning (Figure 1)

As will be shown below, the phase and frequency coordination among $\alpha_{1}$-motoneuron firings can also directly be seen in recordings of single-motor unit surface electromyography.

The phase and frequency coordination are shown in Figure 15 schematically in a recording from a dorsal S4 root of a brain-dead human. Of the summed afferent and efferent impulse traffic, the natural impulse patterns of one $\alpha_{2}$-motoneuron, a dynamic $\left(\gamma_{1}\right)$ and a static $\gamma$-motoneuron $\left(\gamma_{21}\right)$ and two to three secondary muscle spindle afferent fibers could be extracted. The natural stimulations performed were pin-

Relative frequency coordination of the oscillatory firing $\alpha_{2}$-motoneuron $\mathrm{O} 2$ with the firings of secondary muscle spindle afferents and dynamic and static $\gamma$-motoneurons
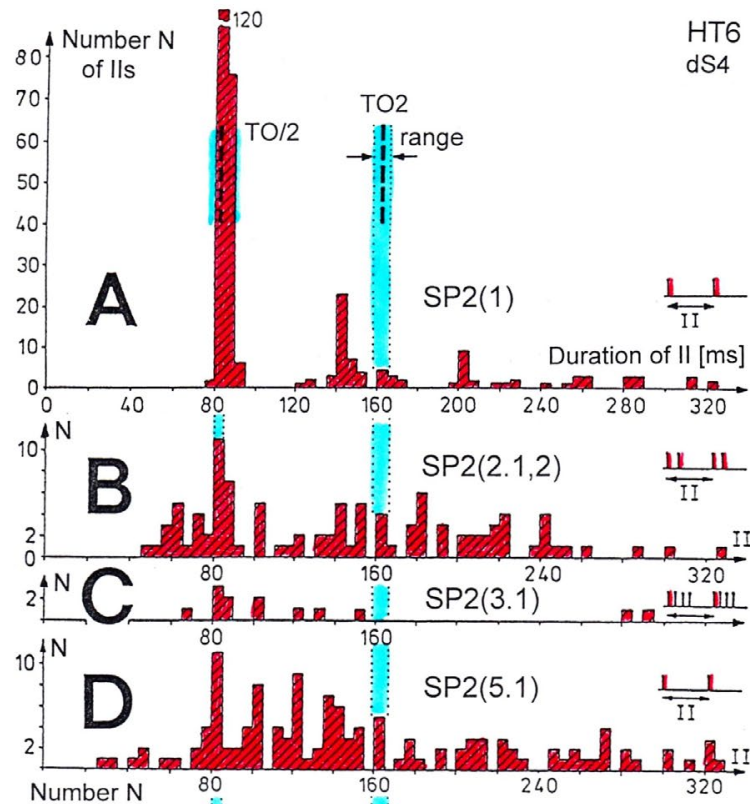

Number $\mathrm{N}$
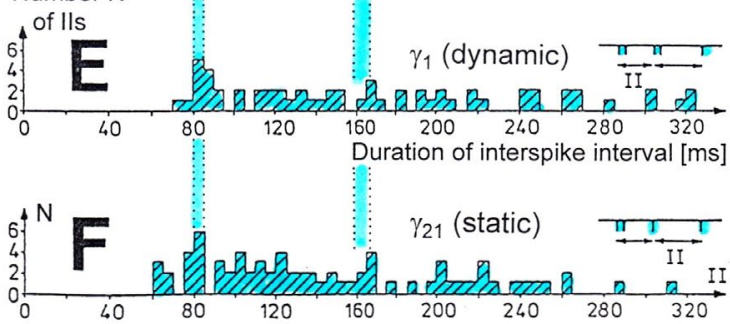

Rhythmic motoneuron and spindle afferent activity

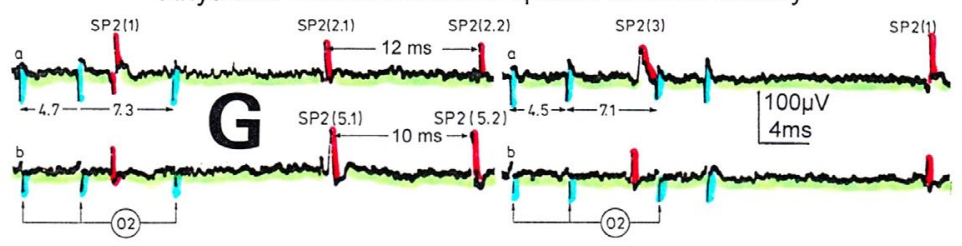

Figure 14. Interspike interval distributions of single endings of four secondary muscle spindle afferents (SP2) and two $\gamma$-motoneurons, recorded simultaneously. In A, the oscillation period TO2 (impulse train length $=3 \mathrm{APs}$ ) with its range of simultaneously recorded oscillatory firing $\alpha 2$-motoneuron $\mathrm{O} 2$ (see $\mathrm{G}$ ) is drawn for comparison; also, the halves of the oscillation period $\mathrm{TO} 2 / 2$ are indicated. Note that the interspike interval distributions of spindle afferents and $\gamma$-motoneurons have shortest interspike interval, nearly identical to the half of the oscillation period (relative frequency coordination). The schematic impulse pattern in A to F shows the procedure for measuring the interspike intervals. Original records of the firing patterns of $\alpha 2-$ motoneuron $\mathrm{O} 2$ and the secondary muscle spindle afferents SP2(1), SP2(2), SP2(3) and SP2(5) are shown in G. Brain-dead human HT6, dS4 root 


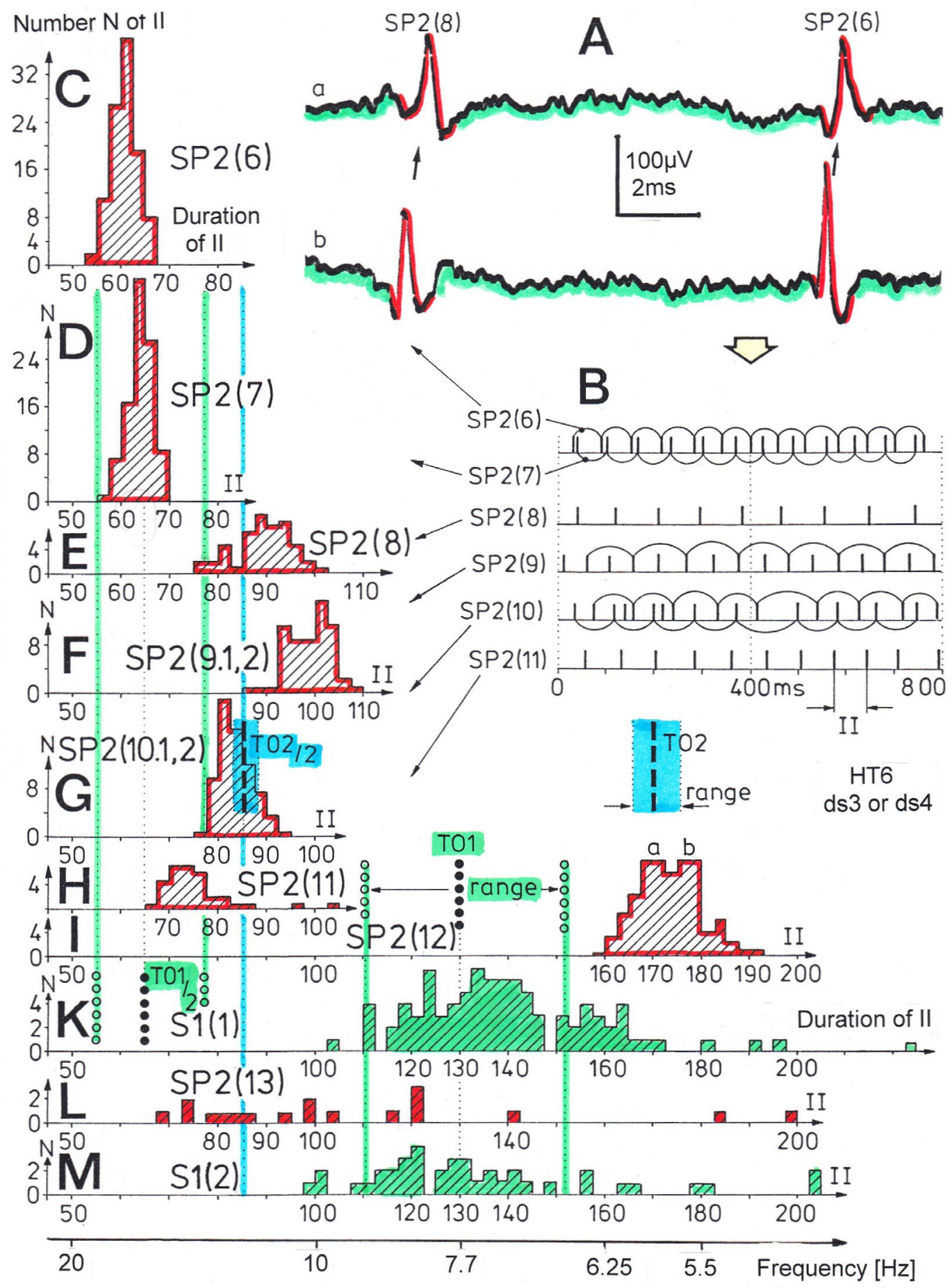

Figure 14A. Measurements from brain-dead human HT6 from different spinal cord segments after retrograde bladder filling (700 to $800 \mathrm{ml}$ ), with the exception of "I", which was obtained before filling. A. Sweep piece of a recording from a dorsal S3 or S2 root filament. It can be seen that the secondary muscle spindle afferent SP2(6) AP can be distinguished by the waveform on the two traces from the secondary spindle afferent fibre SP2(8) AP (different amplitude of the three phases of the triphasic APs). B. Simultaneously recorded impulse patterns of the six parent secondary spindle afferents SP2(6) through SP2(11) obtained from dS3 or dS2 root recordings. The impulse patterns of SP2(6) and SP2(7) fibres are not separated to show the similarity of the patterns. The impulse patterns of the parent spindle afferents SP2(9) and SP2(10) are split into patterns of the single endings (single ending activity partly connected by circle lines) with the assumption that single endings of parent secondary muscle spindle afferents should have interspike intervals of duration longer than $50 \mathrm{~ms}$. $\mathrm{C}$ to $\mathrm{H}$. Interspike interval distributions of six simultaneously recorded single secondary spindle afferent endings. F, G. Interspike interval distributions of parent fibres, which are the sums of the distributions from the two activated endings. I. Interspike interval distributions of a secondary spindle afferent fibre (SP2(12)) of a coccygeal root. K, L, M. Interspike interval distributions of single-fibre afferent activity from a lower sacral dorsal root. In L, most likely the activity from a secondary spindle afferent fibre is shown. In $\mathrm{K}$ and $\mathrm{M}$ most likely the interspike intervals from afferents (S1(1) and S1(2)), innervating stretch receptors of the urinary bladder wall, are shown. In G, H and K, the durations of the oscillation periods (mean and range) of the oscillatory firing a2motoneurons are indicated by thick dashed and dotted lines; the motoneurons innervate the external anal sphincter (TO2) and the external bladder sphincter (TO1). The sites of innervation of the oscillatory firing motoneurons are identified (and distinguished from each other) by anal reflex stimulation, bladder filling and catheter pulling. Note that the TO1 and TO2 ranges and their halves overlap with the interspike interval distributions of the secondary spindle and stretch receptor afferents (relative frequency coordination). 
pricking (pain) of sacral dermatomes inside the continence automatism zone and urinary bladder catheter pulling [3-5]. It can be seen from Figure 15 that the $\alpha_{2}$-motoneuron $\left(\alpha_{2}(\mathrm{O} 2)\right)$ fired in an oscillatory manner with 2 to 3 impulses per impulse train and sometimes there was a break in the oscillatory firing. Phase coordination's between the $\alpha_{2}$-motoneuron, the $\gamma$-motoneurons and the secondary muscle spindle afferent fibers are indicated by different arrows and the dotted and dashed lines. It can be seen from Figure 15 that there were many coordination's between the different neurons. The relative phase and frequency coordination's seem to hold for all neurons and is an integrative mechanism for the self-organization of the neuronal networks of the human CNS (Figure 15).

Because of importance, before going on to the impairment of phase and frequency coordination following CNS injury, malformation or degeneration, phase and frequency coordination among motoneurons will be demonstrated by surface electromyography (sEMG). It will also be shown below that with sEMG the same impulse patterns can be recorded for motor units as was obtained with the single-nerve fiber action potential recording method for the motoneuron axons (Figure 17).

Surface Electromyography to record motor programs, oscillatory firing, and phase and frequency coordination among single-motor units: Another human electro-physiologic tool to measure natural impulse patterns of neurons is the surface electromyography (sEMG). With the same recording system used to record singe-nerve fiber APs, just replacing the wire electrodes with EMG surface electrodes, singlemotor unit firing, and motor programs can be recorded non-invasively. The sEMG recording arrangement is shown in Figure $16 \mathrm{~cd}$ for recording motor programs from an infant.

When surface EMG is performed from a healthy person or child, coordinated motor programs can be recorded from the different muscles (Figure 16). The patterns of recruitment of motor units cannot be seen in such a motor program. Because the number of activated motor units is so high that single motor units cannot be followed up. However, when only a few motor units can be activated in a certain muscle (as for example in spinal cord injury), then the pattern of activation of the motor units and the coordination between them can be seen (Figure 18). If the CNS of a patient is functioning rather physiologically as a result of a long-lasting intensive coordination dynamics therapy, then an analysis of the generation of the motor program becomes possible based on single-motor unit firing.

If we have two electrophysiologic methods to analyze CNS organization, then both methods should give same or similar results. In Figure 17 it is shown that the oscillatory firing of the three motoneuron types are the same. With the single-nerve fiber action potential recording method it can be recorded from single motoneuron axons and afferent fibers. But the method is invasive. With the single-motor unit surface electromyography it can be recorded only from singlemotor units, but the method is non-invasive. Figure 17 compares the oscillatory firing of motoneurons and motor units.

\section{Relative coordination of natural firing patterns of oscillatory firing $\alpha_{2}$-motoneuron, secondary spindle afferents and static and dynamic $\gamma$-motoneurons}

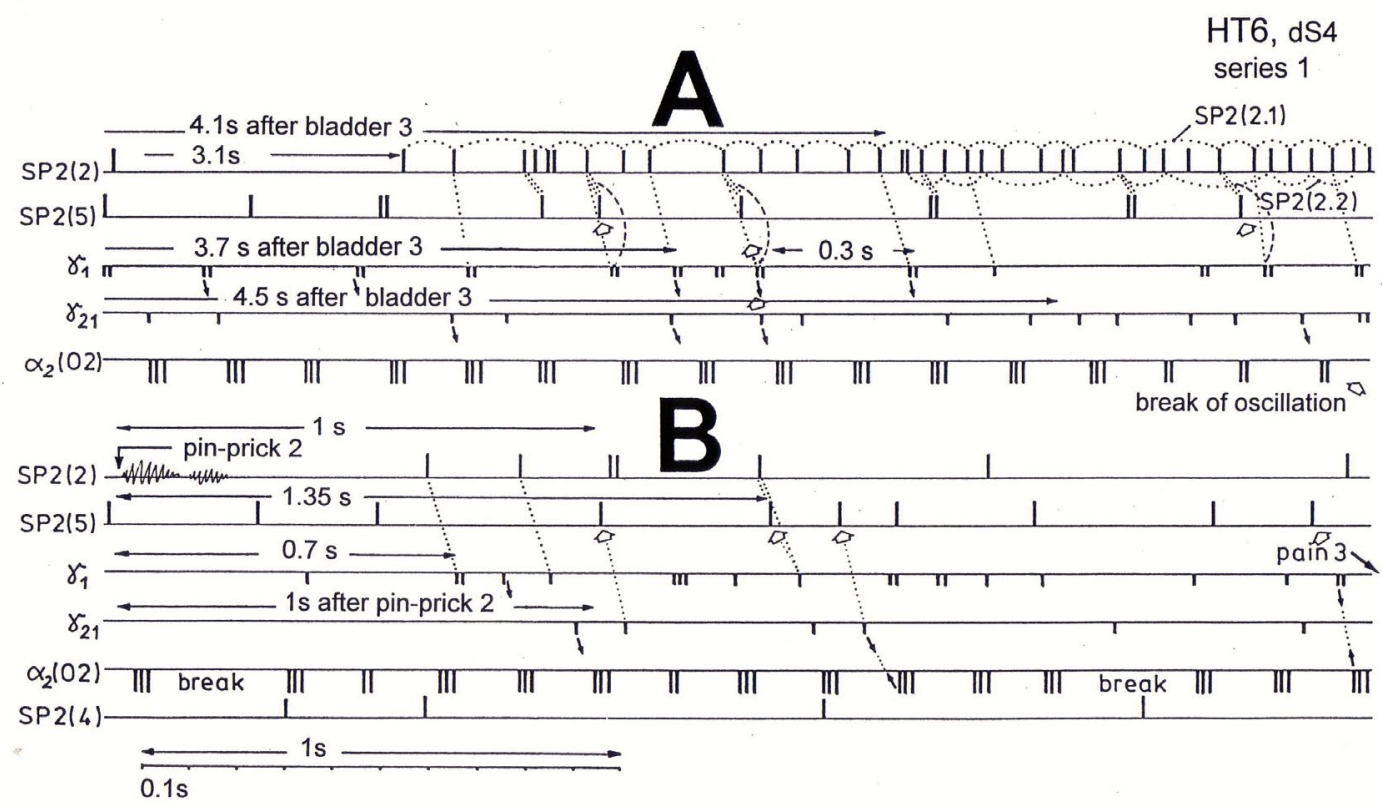

Figure 15. Phase and frequency coordination between the extracellular recorded action potentials of simultaneously recorded $\gamma$-motoneurons $\left(\gamma 1\right.$ and $\left.\gamma_{21}\right)$, secondary spindle afferent fibers (SP2(2), SP2(4), SP2(5)) and oscillatory firing aa ${ }_{2}$-motoneuron $\mathrm{O} 2$ following bladder catheter pulling (bladder 3) (A) and pin-prick 2 (B). B was recorded before A. In A the impulse patterns of the 2 encoding sites SP2(2.1) and SP2(2.2) of the single parent fiber SP2(2) are indicated by the dotted curves. Times to the activity increases of $\gamma$-motoneurons and secondary spindle afferents following stimulation are indicated. Similar time intervals of the occurrence of $\gamma$-motoneuron APs and SP2(5) fiber APs (phase coordination) are indicated by the open arrows, and the similar time intervals of $\gamma$-motoneuron APs and aa-motoneuron APs are indicated by small arrows. Similar time intervals of the APs of fibers SP2(2) and SP2(5) are indicated by the double dotted lines, those of $\gamma 1$-APs and the SP2(2) fiber APs by a dotted line, and those of $\gamma 1$-APs and the SP2(2)-SP2(5) correlation by a curved dashed line. HT6; dS4-root 


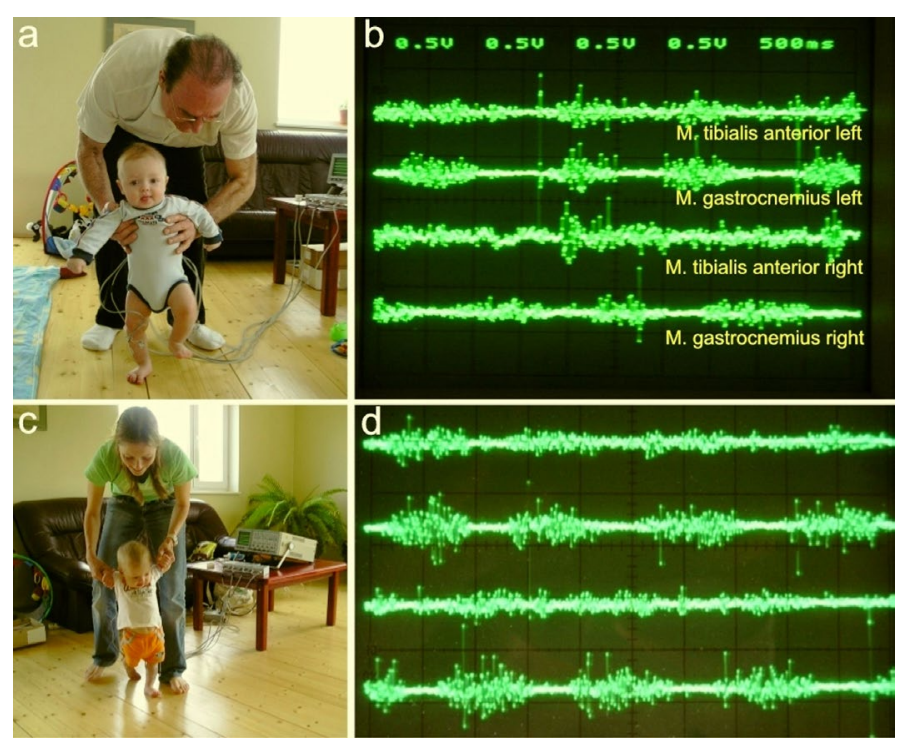

Figure 16. Surface EMG obtained from the healthy 5-months-old (a,b) and 8-months-old old "Jürgen" (c,d) during supported walking by the Author and the mother. a. Walking resembles automatic stepping, because of the strong lifting of the left knee. The toes of the right foot are plantar flexed, which is not physiologic. b. Surface EMG motor programs of left and right tibialis anterior and gastrocnemius muscles. Note that there is no antagonistic action between the tibialis anterior and gastrocnemius muscles. The right tibialis anterior muscle shows no motor program. c. The walking is more walking like and not so much anymore automatic stepping like. d. Better motor programs then 3 months earlier (b). Still there exists no antagonistic action between the tibialis anterior and gastrocnemius muscles. The activation of the right tibialis anterior muscle is a bit better than 3 months ago (b)

The different frequency patterns of oscillatory firing of the three kinds of motoneurons are shown in (Figure 17). Original records were taken with the single-nerve fiber action potential recording method from motoneuron axons and surface electromyography (sEMG) from single motor units. $\alpha_{1}$-Motoneurons innervate FF-type muscle fibers and fire rhythmically with impulse trains consisting of 1 action potential in the order of $10 \mathrm{~Hz}$ (Figures 13). $\alpha_{2}$-Motoneurons innervate FR-type muscle fibers and fire rhythmically with impulse trains consisting of 2 to 5 action potentials in the range of 4 to $7 \mathrm{~Hz}$. The amplitude of the extracellular action potential of the $\alpha_{2}$-motoneurons (axon group diameter $=10.2 \mu \mathrm{m}$, axon group conduction velocity $=50 \mathrm{~m} / \mathrm{s}$ ) is on average smaller than that of the $\alpha_{1}$-motoneurons (axon group diameter $=13.1 \mu \mathrm{m}$, axon group conduction velocity $=65 \mathrm{~m} / \mathrm{s}$ ) (Figure 8 ), depending on the position of the axon in the nerve root with respect to the recording electrodes. FR-type motor unit potentials have much smaller amplitudes than the motor unit potentials of FF-type muscle fibers. The $\alpha_{3}$-motoneurons (axon group diameter $=8.3 \mu \mathrm{m}$, axon group conduction velocity $=37 \mathrm{~m} / \mathrm{s}$ ) innervate S-type muscle fibers and fire oscillatory at a frequency of around $1 \mathrm{~Hz}$ with long impulse trains (up to 50 action potentials per impulse train). The motor unit firing of single S-type muscle fiber motor units could not be safely identified by sEMG because their amplitudes are still smaller than those of FRtype motor units and are thus difficult to identify. The impulse patterns of oscillatory firing motoneurons obtained with sEMG are similar or the same as those obtained with the single-nerve fiber action potential recording method. This confirms the accuracy of the single-nerve fiber action potential recording method. Since sEMG is a non-invasive recording method, oscillatory firing can be recorded easily when using appropriate patients.

With the single-nerve fiber action potential recording method urinary bladder functions were successfully analyzed, whereas surface EMG was very helpful to analyze locomotion and movements. With the single-motor unit sEMG the coordinated firing between motor units will now be shown (Figure 18).

\section{Phase and frequency coordination's between $\alpha_{1}$-motor unit firings of different muscles and different arms:}

Relative phase and frequency coordination for motor unit firings can be shown in the same muscle. Figure 18 shows phase and frequency coordination among motor units between different muscles and arms in a spinal cord injury patient (sub C4/5). A single FF-type motor unit of the right flexor carpi radialis muscle fired at approximately $8 \mathrm{~Hz}$ when activated on volition (Figure 18A). In Figure 18B, the same motor unit is seen firing at $12.5 \mathrm{~Hz}$. This motor unit fired in relative coordination (marked with the arrows) with another single FF-type motor unit in the left flexor carpi radialis muscle. The phase relations have different values in 'A' and ' $\mathrm{B}$.' However, also note that the motor unit in the flexor changed its oscillatory firing from $6.7 \mathrm{~Hz}$ to $10 \mathrm{~Hz}$, probably to develop more motor unit force. Not every motor unit potential is correlated. There may be correlations to motor units of other muscles which were not recorded from. In Figure 18C, phase relations are indicated with the arrows from the right extensor carpi radialis to the left extensor carpi radialis. In Figure 18D, phase relations are indicated by the arrows from the left extensor carpi radialis to the left flexor carpi radialis. Thus, there is coordinated firing between all motor units activated on volition, when the patient is not exercising on the special coordination dynamics therapy device but is in position to do so.

During exercise on the special coordination dynamics therapy device at $5 \mathrm{~N}$, a motor program was partly generated in the right and left extensor carpi radialis muscles (Figure 18E, F). In the right flexor carpi radialis muscle, the CNS tried to build up a motor program with 2 or 3 innervated motor units. In the left flexor carpi radialis muscle no motor program was generated, even though small motor unit potentials can be seen (marked by the arrows in Figure 18E) (Figure 18).

\section{Human pathophysiology}

Following CNS injury, malformation or degeneration, often the rhythmic firing of motoneurons becomes obvious, especially in patients with Parkinson's disease. The impaired phase and frequency coordination results in pathologic neural network organization and pathologic patterns.

Impaired phase and frequency coordination among $\alpha$ and $\gamma$-motoneurons and secondary muscle spindle afferents, measured with the single-nerve fiber action potential recording method: Following brain or spinal cord injury, the phase and frequency coordination between neuron firing becomes impaired [25]. Also, in degeneration, malformation or ageing this coordinated firing of neurons is or becomes impaired and has to be repaired.

In the somewhat healthy case (with respect to the spinal cord) of a brain-dead human, the variability of phase coordination is small (Figure 19). Following spinal cord injury, the variation of phase coordination increases (Figure 20). For further details see [3,4]. Following injury, the variability of phase coordination increases therefore strongly.

A tremendous increase of the Eigenfrequency band of $\alpha_{2}$ oscillators following injury is shown in Figure 21. In the case of a brain-dead human the Eigenfrequency band is small and in the healthy case the variation of the Eigenfrequency is very small as is suggested in Figure 21. But following spinal cord injury the Eigenfrequencies of the $\alpha_{2}$-premotor spinal oscillators vary strongly (Figure 21). With such an Eigenfrequency variation, frequency 


\section{Oscillatory firing of motoneurons of the human spinal cord}

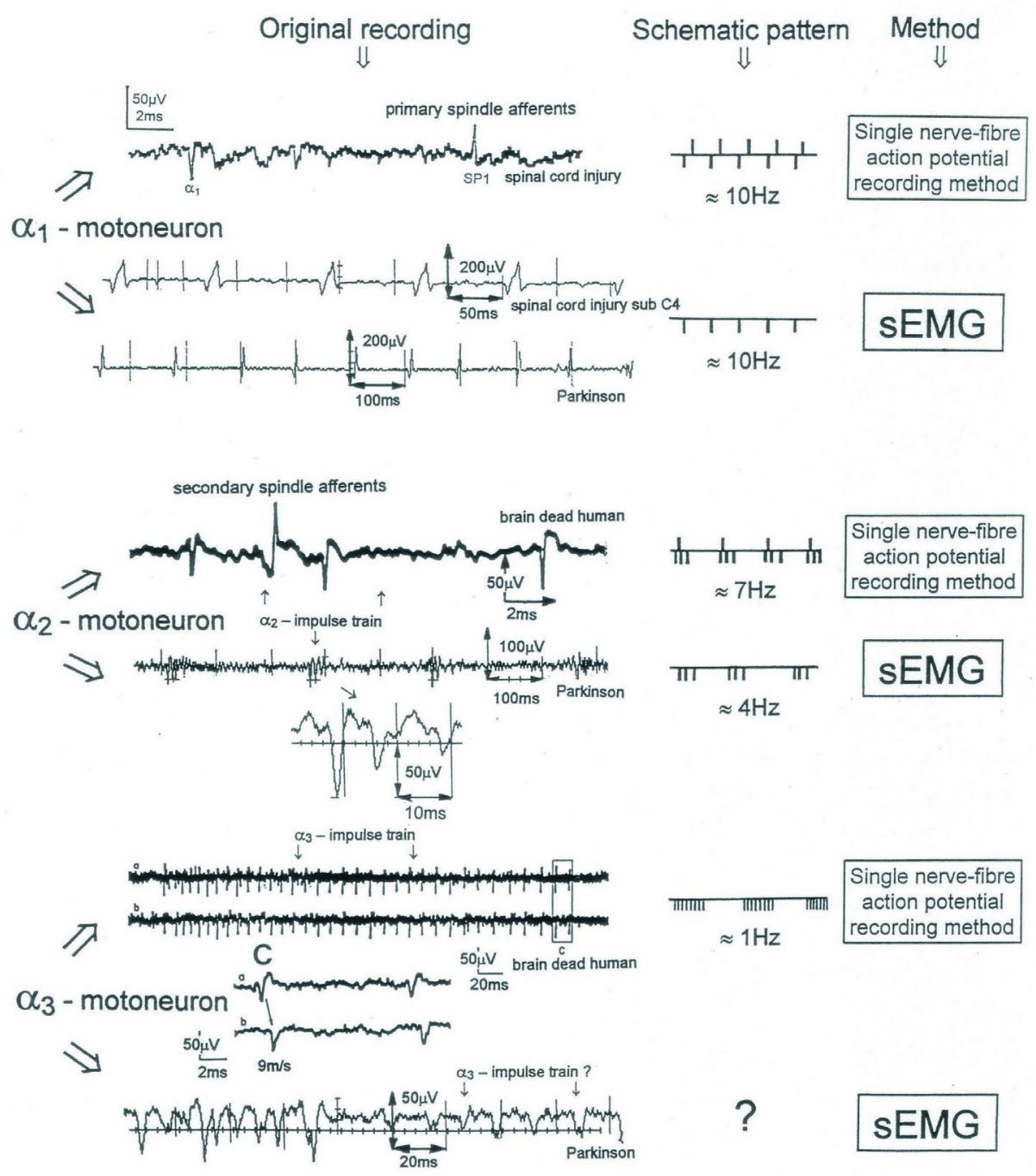

Figure 17. Oscillatory firing patterns of $\alpha_{1}, \alpha_{2}$, and $\alpha_{3}$-motoneurons recorded from motoneuron axons with the single-nerve fiber action potential recording method and the single-motor unit surface electromyography (sEMG) from FF, FR, and S-type motor units. The left panel shows original recordings, the middle panel the schematic patterns; the recording methods are indicated on the right side. The recordings were taken from patients with spinal cord injury and Parkinson's disease and from brain-dead humans

coordination becomes strongly impaired and continuous pathologic synchronization easily occurs as for example in Parkinson's disease. The specificity of network organization becomes strongly impaired.

Motor program bursts in patients with Parkinson's disease structured with tremor activity and motor unit oscillatory activity, recorded by sEMG: With the impaired phase and frequency coordination, many kinds of pathologic coordination's take place in the neural networks, as spontaneous oscillatory firing and uncontrolled synchronized firing of subnetworks.

The impaired coordination leading to spontaneous oscillatory firing and synchronized oscillatory firing of FR and FF-type motor units and tremor can be seen during movements in patients with Parkinson's disease [26].

In the motor program bursts shown in Figure 22A, rhythmic activity can be identified, which cannot be seen in the rather physiologic motor pattern (Figure 16). The highlighted rhythmic firing at 11.4 and $11.8 \mathrm{~Hz}$ may indicate oscillatory firing of FF-type motor units innervated by $\alpha_{1}$-motoneurons.

In another, more time-stretched motor program recording, such rhythmic firing can be seen more clearly (Figure 22B). Rhythms of 5 and $4.2 \mathrm{~Hz}$ were most likely induced by oscillatory firings of an $\mathrm{\alpha}_{2}$ motoneuron, and those of 9.5 and $11 \mathrm{~Hz}$ were most likely induced by oscillatory firings of an $\alpha_{1}$-motoneuron.

Three important conclusions can be drawn from Figure 22. Tremor not only occurs during rest, but also during movement. The reduced intensity of tremor during movements may indicate entraining of neuronal networks resulting in a reduction of tremor in the short-term memory (see below). Second, FF and FR-type motor units, innervated by $\alpha_{1}$ and $\alpha_{2}$-motoneurons respectively, oscillate with their 'Eigen-frequencies' for high activation during 


\section{Phase and frequency coordination between motor unit firing and development of a motor program}

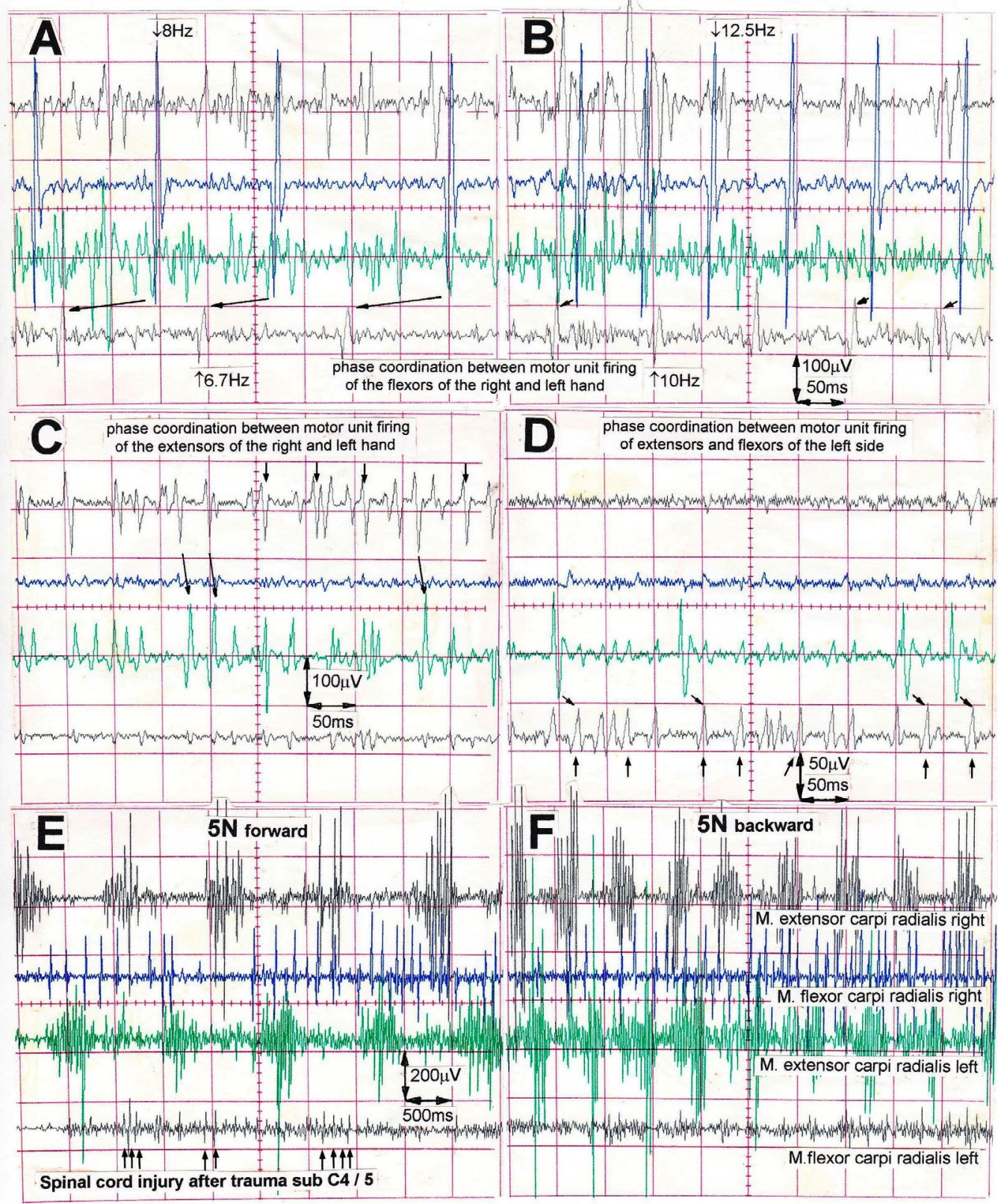

Figure 18. Phase and frequency coordination of single- $\alpha$-motor unit firing between different muscles and between different arms. Phase coordination's are indicated by the arrows between motor unit potentials. A-D. The patient with a spinal cord injury was in position at the special coordination dynamics therapy device but activated muscles on volition upon looking onto the oscilloscope screen. E,F. Patient exercising on the special device. Note the beginning of the appearance of a motor program in the right flexor carpi radialis muscle for $5 \mathrm{~N}$ forward turning. Recordings for a patient with a complete spinal cord injury sub $\mathrm{C} 4 / 5$

motor program burst. Third, since synchronized oscillatory firing in tremor can also be seen in motor bursts during movements, two organizations, namely tremor and movement were organized in the neuronal networks at the same time.

When the patients exercised coordinated arm and leg movements on a special coordination dynamics therapy device (Figure 1), rhythmic activity can be observed in the motor bursts for Parkinson's disease patients (Figure 22) and for patients who suffered a spinal cord injury. First, this rhythmic activity during motor bursts supports the conclusion that during the motor burst, highly activated motor units fire in an oscillatory manner. Second, this partly synchronized and therefore uncoordinated uncontrolled rhythmic activity was still partly controlled by coordinated afferent input, as rhythmic firing was only present during the motor burst and did not occur continuously. The partial control of tremor activity was further indicated by the fact that in different muscles, the tremor was mostly coordinated with respect to agonist and antagonistic muscles, depending on the rhythmic movements of arms, legs and fingers.

The triggering mechanisms of Parkinsonian tremor and largescale coordination: In Figure 23A, B two tremor starting mechanisms 


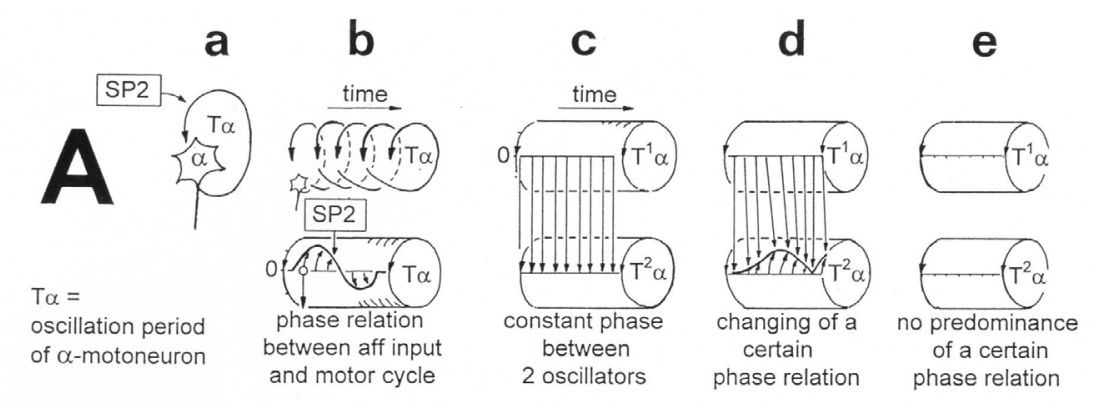

Phase relations between the $\alpha_{2}$-motoneuron oscillation cycle $\left(T \alpha_{2}\right)$ and
muscle spindle afferent and efferent fibres

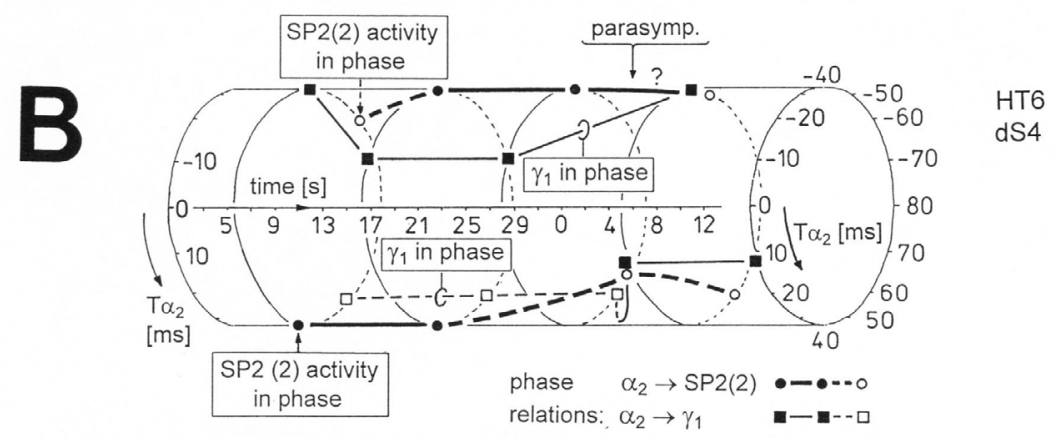

Figure 19. A. Derivation of the simultaneous description of interspike intervals and phase relations. a,b. The oscillation period of an oscillatory firing $\alpha$-motoneuron is schematically characterized by the length of the loop (perimeter). Successive oscillation periods with ongoing time yield a cylinder. Flashing with a stroboscope on such a cylinder with the same frequency as that of the rotation of the cylinder would make a black spot on the turning cylinder not move up or down. If the frequency of the cylinder or the stroboscope changes slowly, the black spot would move up or down. If the black spot moves from left to right with ongoing time, a curve is obtained. By replacing the flashing of the stroboscope by the occurrence of the APs of the spindle afferent fiber (or another oscillatory firing motoneuron) with respect to the APs of the oscillatory firing motoneuron, phase relation changes are made visible in the lower part of 'b' for a constant oscillation period (cylinder with no diameter changes). (c) A constant phase between two oscillatory firing motoneurons results to a constant line on the cylinder with ongoing time. (d) A changing phase gives a curve on the cylinder circumference. (e) If there is a loss of predominance of a certain phase between two motoneurons (the black spot gets diffused with ongoing time and is then lost) there is no line or curve.

B. Interspike interval and phase data from the brain-dead human HT6 (root dS4) are plotted in the representation of A. Filled dots and squares represent average phases (phase relations); thick and thin lines connect the dots to show trends. Note that the phase relations change only little; the frequency of the sphincter $\alpha_{2}$-motoneuron $\left(1 / \alpha_{2}\right)$ changes only little - the cylinder does not change its diameter

Phase relation changes between the cycles of oscillatory firing motoneurons and the spindle afferent fibre SP2(1)
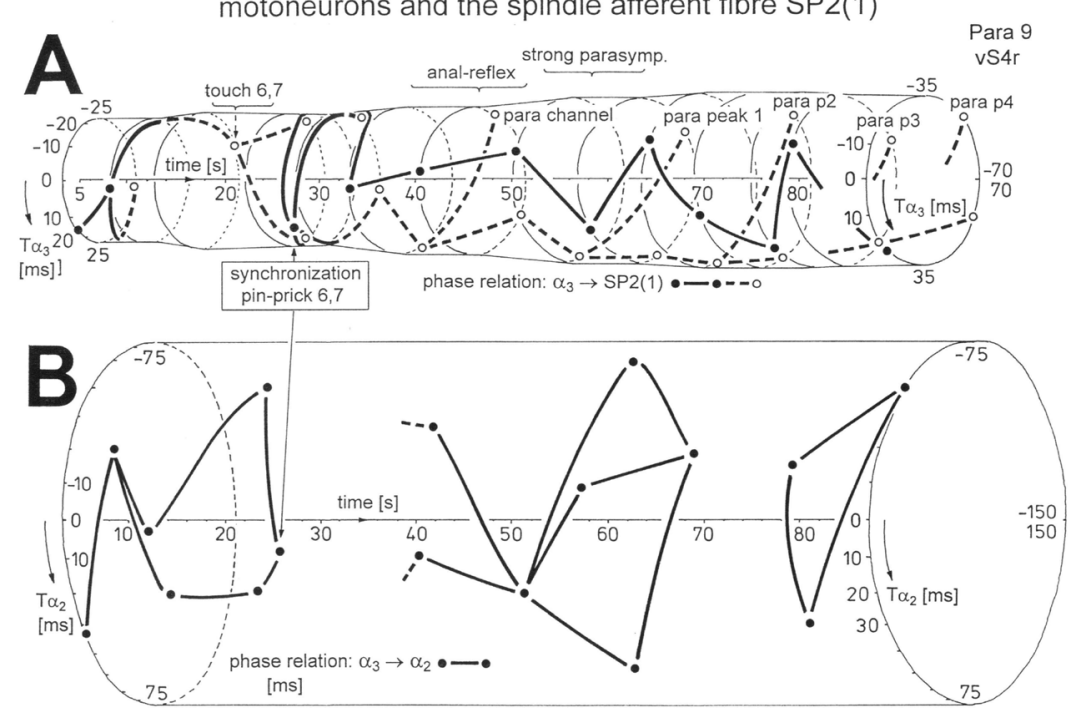

Figure 20. A. Phase relations between the secondary muscle spindle afferent fiber SP2(1) and the oscillatory firing $\alpha_{3}$-motoneuron, are plotted on the oscillation period cylinder T $\alpha_{3}$. The cylinder is changing its diameter (perimeter) because the oscillation period changes. Phase changes in ms are scaled on the cylinder circumference. The ongoing time (to the right) is scaled on the axis of the cylinder. Existing phase relations are represented by dots (filled and open (back-side)); lines (filled and dashed (back-side)) only connect the phase relations to show trends. para peak 1, para $\mathrm{p} 2$, para $\mathrm{p} 3$, para $\mathrm{p} 4=$ activity peaks of the SP2(1) fiber due to parasympathetic activation. B. Phase relations between the $\alpha$ and $\alpha$-motoneurons plotted onto the oscillation period cylinder of the $\alpha_{2}$-motoneuron. Dots represent phase relations. Note that the phase relations of the paraplegic 9 are much more variable than those of the brain-dead human HT6 (Figure 19); also, the number of phase relations changes. For further details see [3,4] 


\section{Distribution of oscillation frequencies in normal, brain-dead and paraplegic humans}

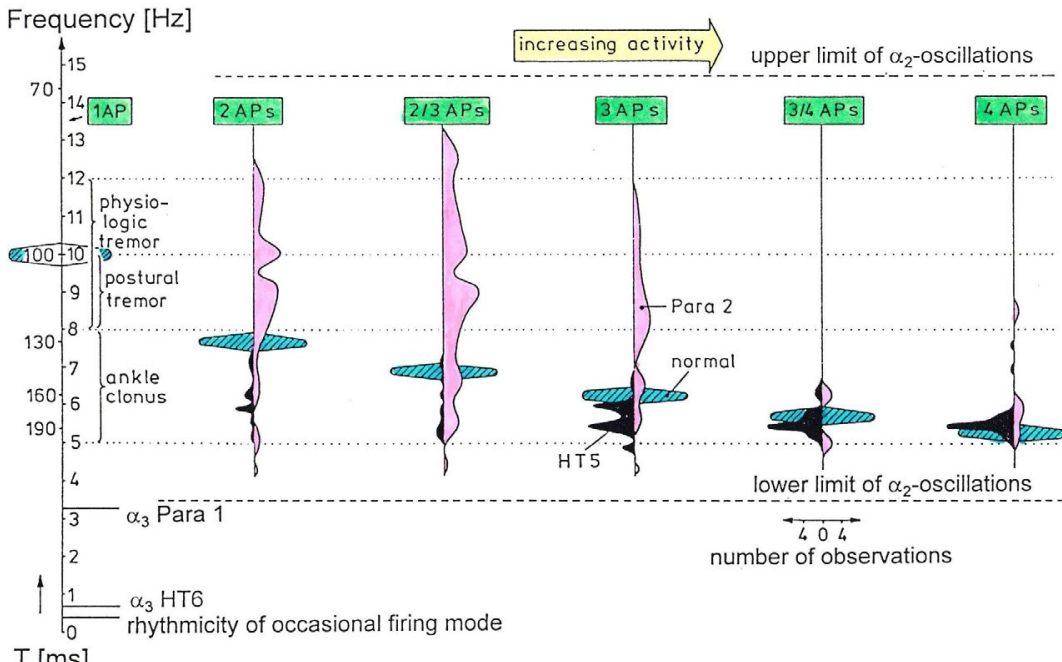

Figure 21. Frequency distributions of oscillation frequencies of continuously oscillatory firing $\alpha_{2}$-motoneurons with increasing number of APs per impulse train (increased activity) in paraplegic 2 (open), in brain-dead HT5 (filled), and probably normal human (cross-hatched). Frequencies and rhythmic activity changes in the occasional and oscillatory firing mode are indicated. Ranges of physiologic tremor, postural tremor and ankle clonus are also drawn. Note that the frequencies for the brain-dead HT5 are too low, and the oscillation frequencies of the spinal cord isolated for a long time (Para 2) are too high and too spread as compared to the theoretically predicted frequency ranges (cross-hatched). $\mathrm{T}=$ oscillation frequency period. $\alpha_{3}$ Para 1 is probably an injured $\alpha_{2}$-motoneuron oscillator

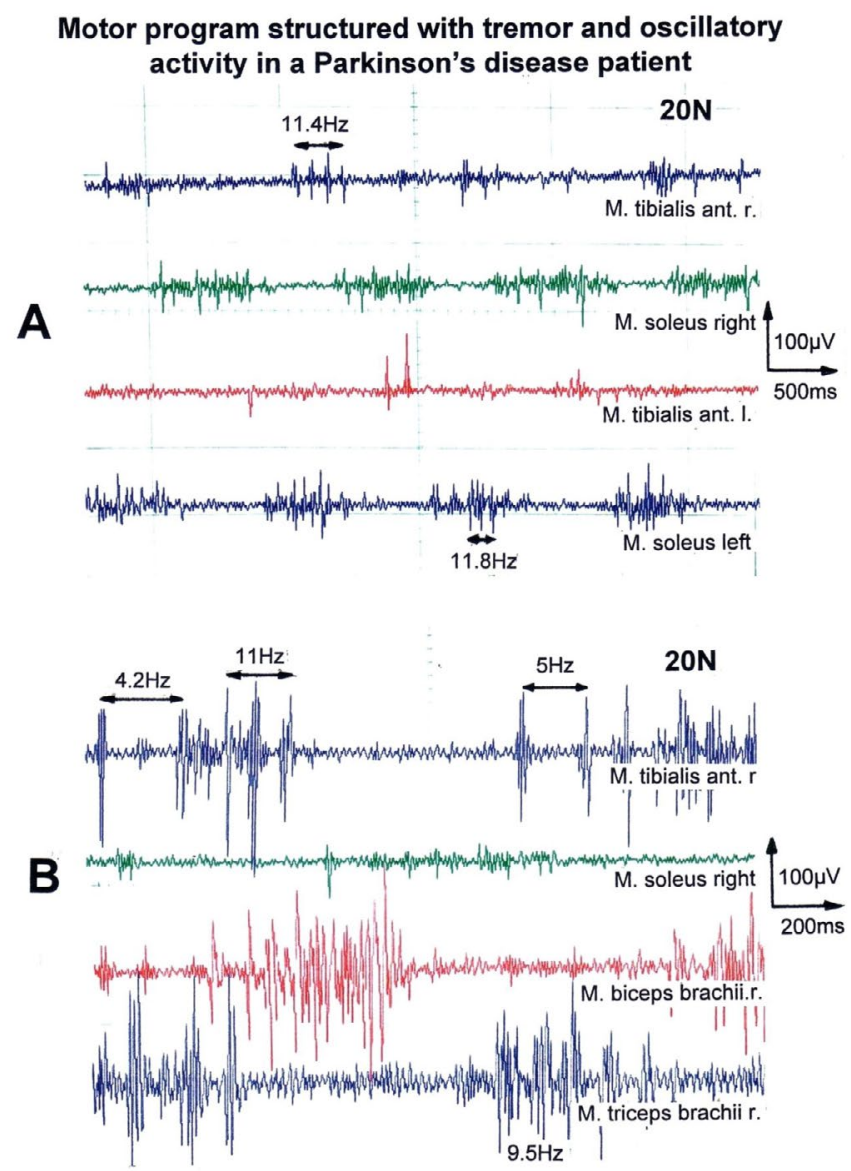

Figure 22. sEMG motor programs of a patient with Parkinson's disease during the exercise on the special coordination dynamics therapy device at a low load of 20N. The motor program muscle bursts are more or less structured by rhythmicity (no rhythmic structure occurs in normal motor bursts). In A, rhythmic activity at a frequency of 11.4 and $11.8 \mathrm{~Hz}$ is suggested. In $\mathrm{B}$, (faster sweep and partly different muscles) low-frequency rhythmicity of 4.2 and $5 \mathrm{~Hz}$ (frequency range of $\alpha_{2}$-motoneuron oscillatory firing) and higher frequency rhythmicity of 11 and $9.5 \mathrm{~Hz}$ (frequency range of $\alpha_{1}$-motoneuron oscillatory firing) are suggested to be seen 
are shown, namely that FR-type motor units started to oscillate with little or no activation (first triggering mechanism) and that oscillatory firing FR-type motor units synchronized their firing (second triggering mechanism). The patient was positioned on a chair at the special coordination dynamics therapy device in the sitting position but was not exercising. In Figure 23A one FR-type motor unit in the gastrocnemius muscle (innervated by an $\alpha_{2}$-motoneuron) started to oscillate (first triggering mechanism), i.e. fired rhythmically with an impulse train consisting of 3 motor unit potentials. Most likely, the gastrocnemius muscle was not moving. Then more FR-type motor units and maybe some S-type motor units started to fire synchronously (Figure 23C). The gastrocnemius muscle may have moved a bit with a frequency of $4.1 \mathrm{~Hz}$. The triceps brachii muscle moved, because a movement artifact was recorded from it (Figure 23C). With the start of FF-type motor unit firing, synchronized with the FR-type motor unit firing (Figure 24A, B and Figure 23E, F, tibialis anterior $\mathrm{r}$.), the muscle moved rhythmically.

A second tremor or muscle movement triggering mechanism concerned FR-type (and may be S-type) motor units synchronizing their oscillatory firing. In the left part of Figure 23B the FR-type motor units oscillated but not in synchronization; no movement artifact at the electrodes of the triceps brachii muscle was recorded. When the FR-type motor units started to oscillate in a synchronized manner at a frequency of $4.2 \mathrm{~Hz}$ (right part of Figure 23B), then a rhythmic movement artifact $(\mathrm{f}=4.8 \mathrm{~Hz})$ was generated at the surface electrodes of the triceps brachii muscle. Since the triceps brachii muscle is sited in the arm and the gastrocnemius muscle in the leg, the oscillatory firing networks of the arm (network assemblies in the intumescentia cervicalis) and the leg (assemblies in the intumescentia lumbosacralis) did communicate with each other. There were therefore large-scale synchronization and coordination at least between the intumescentia cervicalis and lumbosacralis, which is a distance of approximately $300 \mathrm{~mm}$.

The full muscle movement or tremor was generated when the FF-type motor units (innervated by $\alpha_{1}$-motoneurons) synchronized their firing with the firing of the FR-type motor units (Figure 23E, F) (third triggering mechanism). With the synchronization of the FF-type motor units, the muscle movement or tremor frequency changed to match frequency coordination between the driving oscillatory firing of $\alpha_{1}$ and $\alpha_{2}$-motoneurons. In Figure 23D, the common frequency of the synchronized oscillatory firing $\alpha_{2}$-motoneurons (and maybe $\alpha_{3}$-motoneurons) was $3.3 \mathrm{~Hz}$. With the synchronization of the $\alpha_{1}$ motoneurons, the common synchronized frequency increased to $3.6 \mathrm{~Hz}$ (Figure 23F). The $\alpha_{1}$-motoneurons could have fired in an oscillatory manner at a frequency of $7.2 \mathrm{~Hz}$, so that a single FF-type motor unit potential could have fired in coordination with every second muscle activation burst of the tremor. If another $\alpha_{1}$-motoneuron oscillated at $10.8 \mathrm{~Hz}$, the innervated FF-type motor unit potential could have fired in coordination with every third activity burst (Figure 23).

In conclusion, in this patient the Parkinsonian tremor started with the synchronized oscillatory firing of FR-type motor units (innervated by $\alpha_{2}$-motoneurons). With the synchronized firing of FF-type motor units (innervated by $\alpha_{1}$-motoneurons) the full muscle movement or tremor was generated.

The reason that the FF-type motor units synchronized their rhythmic firing and that the FF-type motor units synchronized their firing with those of the FR-type motor units and not vice versa is that $\alpha_{2}$-oscillators have a high and $\alpha_{1}$-oscillators a low oscillator stability.
Synchronization and de-synchronization of FF-type motor unit firing with oscillatory firing FR-type motor units: In Figure 24, the synchronization and de-synchronization of FF-type motor units (innervated by $\alpha_{1}$-motoneurons) with FR-type motor units (innervated by $\alpha_{2}$-motoneurons) is shown. In Figure 24A, FR and S-type motor units (small motor unit amplitude) fired rhythmically at a common frequency of $3.9 \mathrm{~Hz}$ in the right tibialis anterior muscle. In Figure 24B, C, FF-type motor units (large unit amplitude) fired synchronized with the FR-type motor units; large FR-type motor unit potentials appeared on top of the small FR-type motor unit potentials. The common rhythmic frequency is now 3.4 and $3.3 \mathrm{~Hz}$. It can be seen that the large amplitude FF-type motor unit potentials slightly changed their synchronization phase with respect to the low amplitude FR-type motor unit potentials; sometimes the low amplitude FR-type motor unit potentials started earlier in the activity burst and sometimes they lasted longer than the large FF-type potentials in the burst (Figure 24B,C). In Figure 24D additionally FRtype motor units started to fire rhythmically in the biceps brachii muscle at a frequency of $4.5 \mathrm{~Hz}$. In Figure 24F FF-type motor unit potentials no longer fired in the tibialis anterior muscle, only FR-type potentials were recorded. The rhythmically firing FR-type motor unit potentials in the biceps brachii muscle increased their firing frequency to $5.5 \mathrm{~Hz}$. In this patient the tibialis anterior muscle was only moving up and down when the FF-type motor units fired in addition (Figure 24).

In Figure 24E, the rhythmically firing motor units are displayed on a time-stretched scale. It can be seen that the FF-type motor units had a motor unit action potential duration of approximately $8 \mathrm{~ms}$ (amplitude $\approx 150 \mu \mathrm{V}$ ) and the FR-type motor unit potentials had a duration of $17 \mathrm{~ms}$ (amplitude $\approx 15 \mu \mathrm{V}$ ). The single-nerve fiber action potentials have a much shorter duration (approximately $0.3 \mathrm{~ms}$ ) (Figure 10A).

Contribution of FF and FR-type motor unit firing to the generation of tremor: There is evidence to suggest that the tremor in Parkinson's disease patients starts with synchronized oscillatory firing of FR-type motor units, innervated by $\alpha_{2}$-motoneurons (Figure 23,24), to which then FF-type motor units (innervated by $\alpha_{1}$-motoneurons) synchronize, since the tremor frequencies are in the range of the Eigen-frequencies of oscillatory firing $\alpha_{2}$-motoneurons $(\approx 5 \mathrm{~Hz})$ and the $\alpha_{2}$-premoter spinal oscillators have a higher stability. Premotor spinal oscillators probably consist of a neuronal network, with the motoneuron being a part of it. $\alpha_{2}$-Motoneuron oscillators are less dependent on afferent input and are therefore more stable. Moreover, the Eigen-frequencies of the muscle-limb mechanics, for example of the arm and hand are also within the range of $5 \mathrm{~Hz}$. It then depends on quantitative effects, i.e. which oscillator type contributes to what extent to the rhythmic movement, at which frequencies the arms, hands and fingers shake. Since the contributions of rhythmically firing FF and FRtype motor units changed as did the strength of activation, the tremor frequency varied during the measurements. Since FF-type motor units develop more power, substantial tremor movement was only observed if substantial numbers of rhythmically firing FF-type motor units contributed. After the start of tremor with the synchronization of FRtype motor units, first the small FF-type (and further FR-type) motor units started to fire synchronously, followed by larger FF-type motor units with, on average, larger potential amplitudes. This may indicate recruitment according to the size principle among FF-type motor units. When the tremor ceased the large FF-type motor units stopped firing first followed by the smaller FF-type ones and then also the smallamplitude FR-type motor units. 
Oscillatory firing of FR and S-type motor units as a start of tremor

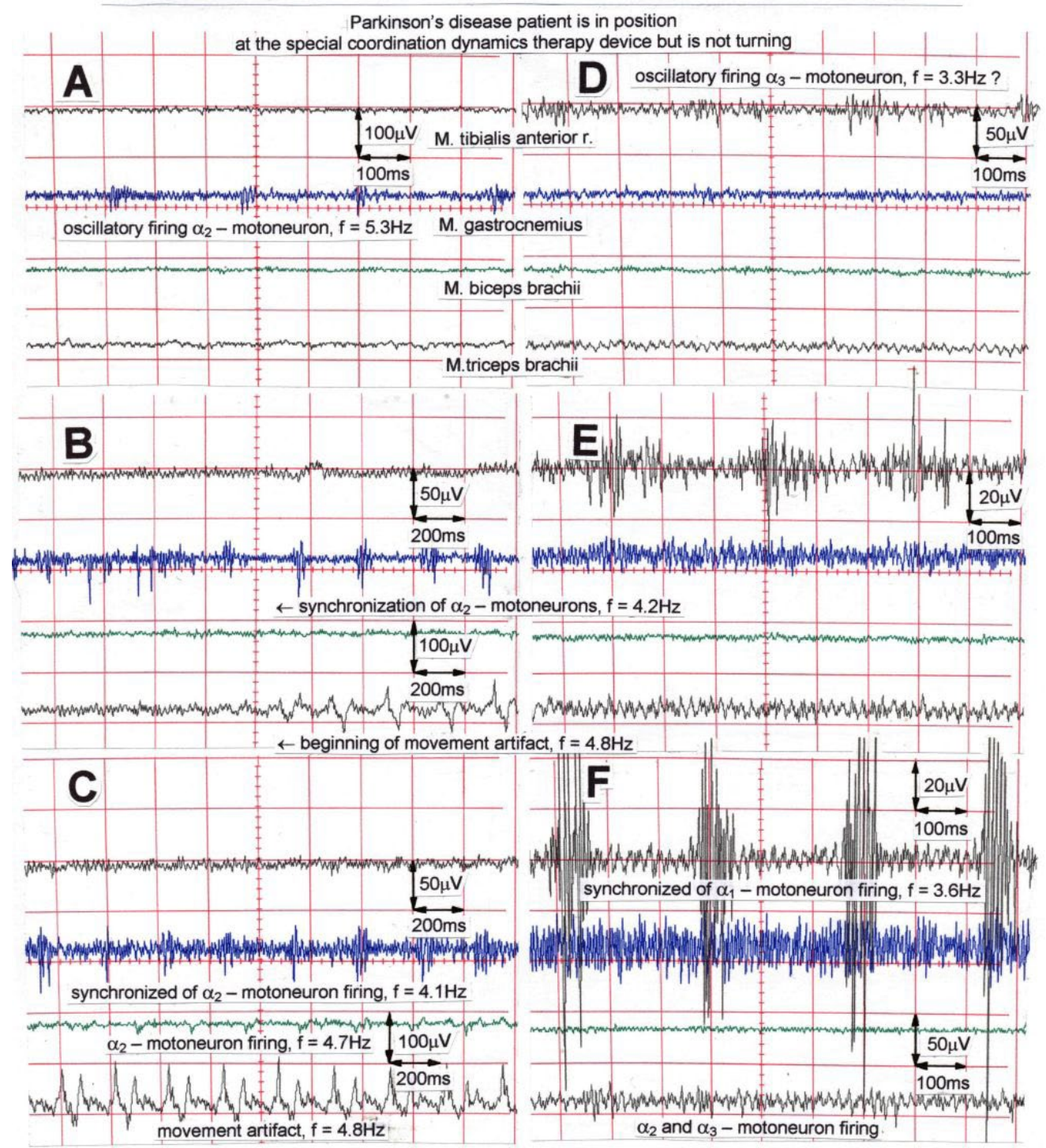

Figure 23. Oscillatory firing of $\alpha_{2}$-motoneurons and the synchronization of oscillatory firing $\alpha_{2}$ and $\alpha_{1}$-motoneurons. Recordings were performed with sEMG. A. Oscillatory firing of a single $\alpha_{2}$-motoneuron motor unit $\left(\mathrm{f}=5.3 \mathrm{~Hz}\right.$; amplitude $\approx 35 \mu \mathrm{V}$, triplet-firing) in $\mathrm{M}$. gastrocnemius. B. Synchronization of mainly oscillatory firing $\alpha_{2}$-motoneuron motor units (FR, $\mathrm{f}=4.2 \mathrm{~Hz}$ ) in gastrocnemius muscle. With the synchronization of the oscillatory firing ${ }_{2}$-motoneurons a movement artifact was induced in the electrodes on M. triceps brachii. C. Synchronized oscillatory firing of $\alpha_{2}$-motoneuron motor units, mixed with a few $\alpha_{1}$-motoneuron motor units of small amplitude in gastrocnemius muscle. In musculus biceps brachii an $\alpha_{2}$-motoneuron motor unit fires at $4.7 \mathrm{~Hz}$ not synchronized with the $\alpha_{2}$-motoneuron firings in gastrocnemius muscle. Rhythmic movement artifact in triceps brachii muscle $(\mathrm{f}=4.8 \mathrm{~Hz})$ indicating an arm tremor of $4.8 \mathrm{~Hz}$. $\mathrm{D}$. Possible oscillatory firing of an $\alpha_{3}$-motoneuron motor unit ( $\mathrm{f}=3.3 \mathrm{~Hz}$; amplitude $\approx 10 \mu \mathrm{V}$; long impulse train) in tibialis anterior muscle; a few $\alpha_{2}$-motoneuron motor unit potentials (larger amplitude) fire synchronized. E. Synchronized oscillatory firing of mainly $\alpha_{2}$ and $\alpha_{3}$-motoneurons $\left(\mathrm{f} \approx 3.3 \mathrm{~Hz}\right.$ ). F. Synchronized firing of ${ }_{1}$-motoneuron unit potentials (FF-type) of large amplitude (up to $200 \mu \mathrm{V}$ ) with the oscillatory firing $\alpha_{2}$ and $\alpha_{3}$-motoneuron motor unit potentials (FR and S-type) in gastrocnemius muscle a few minutes later (resulting frequency $=3.6 \mathrm{~Hz}$ ). Rhythmic firing of $\alpha_{2}$ and $\alpha_{3}$-motor unit potentials in the triceps brachii muscle in some coordination to the rhythmic firing in tibialis muscle giving rise to substantial rhythmic movements of tibialis anterior muscle. D, E, and F are consecutive recordings. Recordings from a 70-year-old patient with Parkinson's disease for 6 years (U.H.); tremor on both sides

Only little information was obtained concerning the contribution of S-type motor units, innervated by $a_{3}$-motoneurons, since their motor unit action potentials seem to be very small to be detected safely by sEMG so far. They even seemed to synchronize their firing with the FR-type motor units. $\alpha_{3}$-Motoneuron oscillators fire in the range of $1 \mathrm{~Hz}$ (Figure 13). Higher-quality recordings are needed to identify S-type motor unit firing safely also by sEMG.

So far, we only have been considering coordination of oscillatory firing motor units. It was observed with the single-nerve fiber action potential recording method that occasionally firing motoneurons had the tendency to coordinate their firing with oscillatory firing motoneurons (page 328 of) [3]. The problem arises how to measure coordination among occasionally firing neurons or motor units since they are difficult to identify in the natural impulse traffic of many neurons or motor units. The only feature to identify occasionally firing neurons or motor units is the waveform. The advantage of oscillatory firing neurons is that additionally they can be identified by the rhythmic firing pattern.

Lack of inhibition as one reason for tremor: It was shown using the single nerve-fiber action potential recording method that $\alpha_{1}$ and $a_{2}$-motoneurons fire oscillatory, that they can synchronize their firing following repetitive stimulation, and that these oscillatory firing 


\section{Activation of $\alpha_{1}-$ motor unit potentials (FF) and their synchronization with oscillatory firing $\alpha_{2}$ - motor unit potentials (FR) to induce tremor in Parkinson's disease patients}

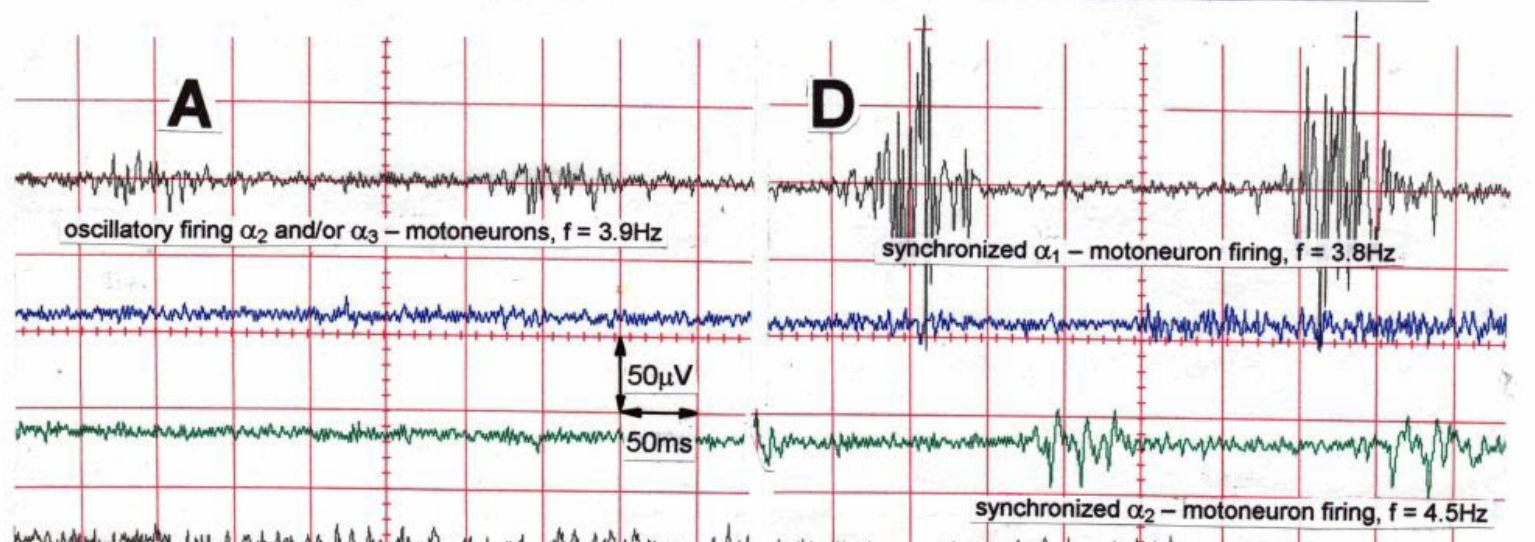

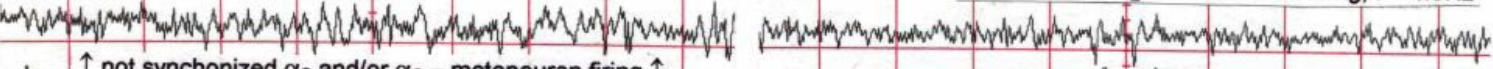

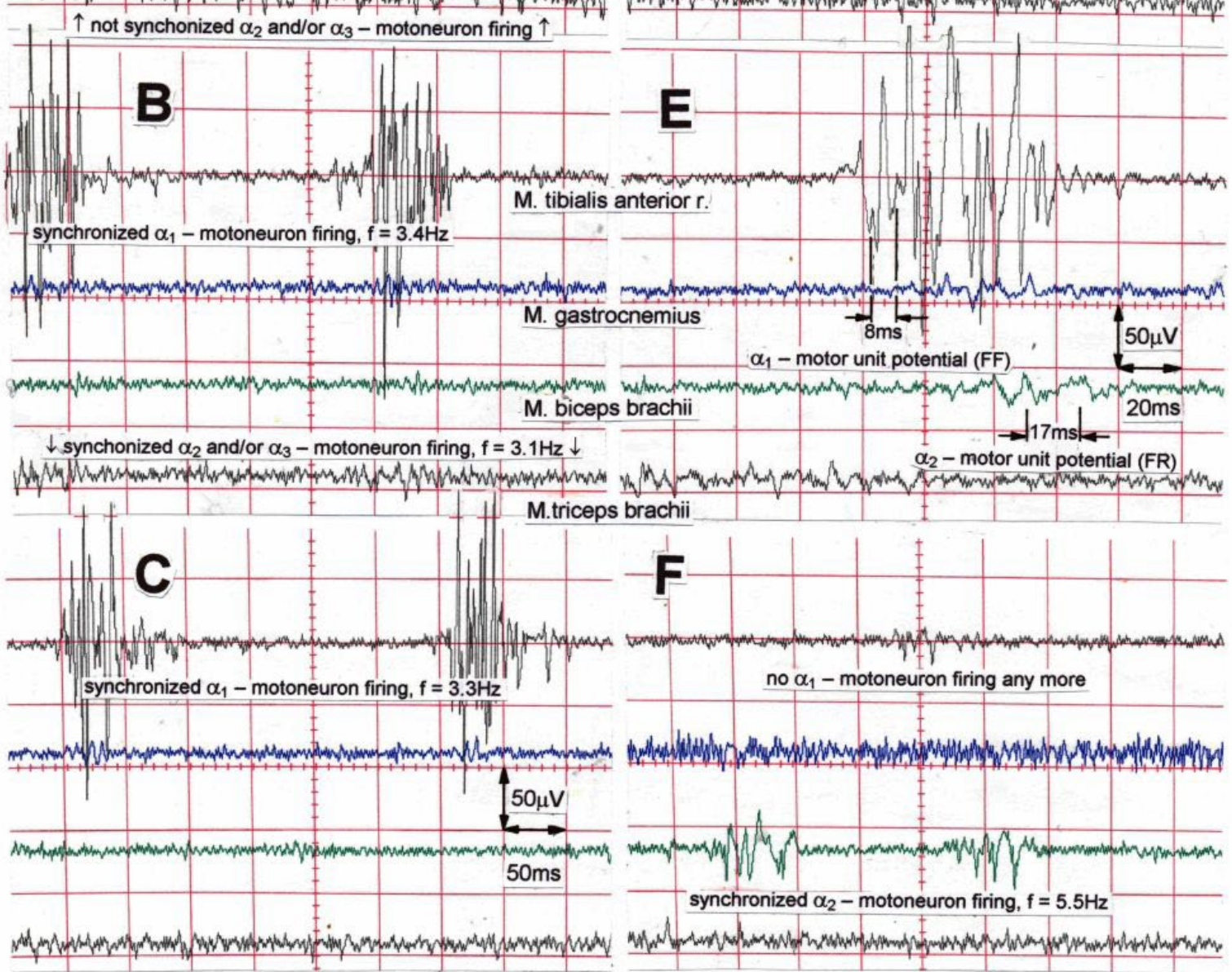

Figure 24. Time course of transient tremor induced by the activation of $\alpha_{1}$-motor unit potentials (FF-type) and their synchronization with oscillatory firing $\alpha_{2}$ and $\alpha_{3}$-motoneuron motor unit potentials (FR and S-type). A. Synchronized oscillatory firing of $\alpha_{2}$ and $\alpha_{3}$-motor unit potentials in tibialis anterior muscle ( $\mathrm{f}=3.9 \mathrm{~Hz}$ ). No synchronized firing of $\alpha_{2}$ and $\alpha_{3}$-motor unit potentials in triceps brachii muscle. No movement of tibialis anterior muscle. B. $\alpha_{1}$-motor unit potentials are activated and synchronized with the $\alpha_{2}$ and $\alpha_{3}$-motor unit potentials ( $\mathrm{f}=3.4 \mathrm{~Hz}$ ). Tibialis anterior muscle moves visibly $\alpha$ and $\alpha$-motor unit potentials in biceps brachii muscle fire partly synchronized with the motor unit potentials in tibialis anterior muscle. $\alpha$ and $\alpha_{3}$-motor unit potentials can be seen before and after the $\alpha_{1}$-motor unit potentials in the activity burst. C. Similar firing as in 'B', but nearly no motor unit firing in triceps brachii muscle. $\mathrm{D}$. Rhythmic firing of motor units giving rise to up and down movement ( $\mathrm{f}=3.8 \mathrm{~Hz}$ ) of tibialis anterior muscle. Synchronized $\alpha_{2}$ and $\alpha_{3}$-motor unit firing in biceps brachii muscle ( $\mathrm{f}=4.5 \mathrm{~Hz}$ ), not coordinated with the rhythmic firing in tibialis anterior muscle. and $\alpha_{3}$-motor unit potentials are activated in gastrocnemius muscle. E. Time-stretched motor unit action potentials: duration of $\alpha_{1}$-motor unit potential $=8 \mathrm{~ms}$ (amplitude $150 \mu \mathrm{V}$ ), $\alpha_{2}$-motor unit potential $=17 \mathrm{~ms}$ (amplitude $\approx 15 \mu \mathrm{V}$ ). F. Cessation of rhythmic firing of $\alpha_{1}$-motor unit potentials in tibialis anterior muscle; no visible contraction of tibialis anterior muscle anymore; some ${ }_{2}$-motor unit firing still occurring. Synchronized $\alpha_{2}$-oscillatory firing still in biceps brachii muscle. Recordings from a 70-year-old patient with Parkinson's disease for 6 years (U.H.); tremor on both sides; measurements were performed on the more affected right side. During the measurements the hands and feet of the patient were in the position for exercising on the special coordination dynamics therapy device 
motoneurons can build up an external loop to the periphery in the way that $\gamma$-motoneurons and muscle spindle afferents get included in the rhythmic coordinated firing (Figure 40B) [3]. But the synchronization of oscillatory firing is only transient, and in non-Parkinson patients the building up of an external loop to the periphery could only be observed upon strong repetitive reflex stimulation. It is therefore concluded that patients with Parkinson's disease lack inhibition, so that motoneurons can start to fire in an oscillatory manner upon virtually no stimulation (spontaneously); and, secondly, they lack mutual inhibition between oscillatory firing motoneurons, so that oscillatory firing motoneurons can synchronize their firing to give rise to rhythmic muscle contractions and movements resulting in tremor.

It is likely that the rhythmic firing during tremor starts with $a_{2}$ motoneurons because their firing is more stable, and their firing frequency range is similar to the Eigen-frequencies of the muscle-limb mechanics. By building up an external loop to the periphery $[3,4]$ by the synchronously firing $\alpha_{2}$-motoneurons, primary muscle spindles are probably also activated, which in turn stimulate $\alpha_{1}$-motoneurons to fire. Inhibiting input to the premotor network will probably first inhibit less stable oscillatory firing $\alpha_{1}$-motoneurons followed by the more stable oscillatory firing $\alpha_{2}$-motoneurons.

There is indication that in patients with Parkinson's disease and in patients who suffered a spinal cord injury, the inhibition necessary to prevent spontaneous oscillatory firing of motoneurons is missing or impaired. A second type of inhibition is missing in Parkinson's disease patients to avoid synchronization among motoneuron firing.

Patients with Parkinson's disease have mainly two functional deficits, namely movement impairment and tremor. The impaired phase and frequency coordination impair their movements like crawling, walking and running. The improvement of movements can be achieved quite quickly through CDT. Often it needs only 1 hour exercising on the special CDT device and the patient can walk better in the short-term memory. But the reduction of tremor is difficult to achieve but possible.

In the next section we are coming to the repair physiology and how to improve the impaired phase and frequency coordination giving rise to tremor and motor program deterioration. For a better understanding of the repair physiology it will be started with System Theory of Pattern Formation. Actually, the combination of the human neurophysiology and the integrative physiology led to the success in brain, brain stem and spinal cord repair.

\section{Human repair physiology}

It is started with the integrative physiology of patterns formation, followed by the understanding and improvement of tremor and motor programs. At the end of the section repair possibilities are summarized.

System Theory of Pattern Formation for understanding neuronal network organization and learning:

To understand the on-going changes of movement and other patterns in healthy humans and in patients with CNS injury, malformation and degeneration (aging), the System Theory of Pattern Formation is used. In a complex system like the human CNS, patterns are generated by a nervous system which seeks cooperative stability. Stability is what defines collective states. The system has the tendency to slip into the collective states to which it is attracted. When an infant crawl (Figure 25), its arms and legs are strongly attracted to the 'pace' and 'trot' gait coordination patterns. The attraction is so strong that intermediate crawling patterns seemingly do not exist, as if the patterns are hard-wired. But with the help of the special CDT device, the CNS can generate intermediate coordination patterns. A patient with a CNS injury often crawls with intermediate arm and leg coordination patterns and has to re-learn the pace and trot gait coordination's for CNS repair and shifts in this way the attractors for crawling to the pace and trot gait coordination's. Attractive states and attractors of CNS organization can be pictured as a ball in a potential well or more generally in an attractor layout (Figure 26). Changes in CNS functioning are characterized as continuous stabilization and destabilization, over time, of preferred attractor states.

To reduce for understanding the complexity of human neural networks of the many billions of neurons, order parameters or collective variables are introduced for the generation of certain movements. An equation of motion describes the coordination patterns dynamics. However, coordination patterns are not only determined by the task or biological function. Patterns adjust continuously to requirements from the environment (transmitted by impulse patterns from stimulated receptors in the periphery), memory, intention, and support given by a therapist. The specific requirements are captured by the concept of behavioral information and are made part of a vector field that attracts toward the required patterns. The coordination pattern dynamics, characterized by equations of motion of collective variables (the vector $\mathbf{X}$ ), takes the general following form [27].

$$
\mathrm{d} \mathbf{X} / \mathrm{dt}=\mathbf{F}_{\text {intr }}(\mathbf{X})+\sum \mathrm{c}_{\text {inf }} \mathbf{F}_{\text {inf }}(\mathbf{X}, \mathrm{t})
$$

where $\mathbf{F}_{\text {intr }}$ designates the intrinsic dynamics of the nervous system. These intrinsic dynamics capture the anatomical (neuronal network structure), physiological and pathological states of the CNS and its muscular-skeletal elements. $\sum \mathrm{c}_{\text {inf }} \mathbf{F}_{\text {inf }}(\mathbf{X}, \mathrm{t})$ represents the sum of external influences $\left(\mathbf{F}_{\text {inf }}(\mathbf{X}, \mathrm{t})\right)$ with their relative strength $\left(\mathrm{c}_{\text {inf }}\right)$ pertaining to each influence. The so-called behavioral information $\mathbf{F}_{\text {inf }}(\mathbf{X}, t)$ includes cognitive states, emotional states, intentions, motivations, instructions, inter-personal coordination, movement support etc. During motor learning or while applying therapy to a patient these extrinsic influences become extremely important, because the intrinsic (pattern) dynamics can be changed with these extrinsic influences by altering the equation of motion. By modulating the behavioral information, the intrinsic dynamics of the neuronal networks can be influenced further, that is if CDT is no longer efficient in repairing the injured CNS, requiring

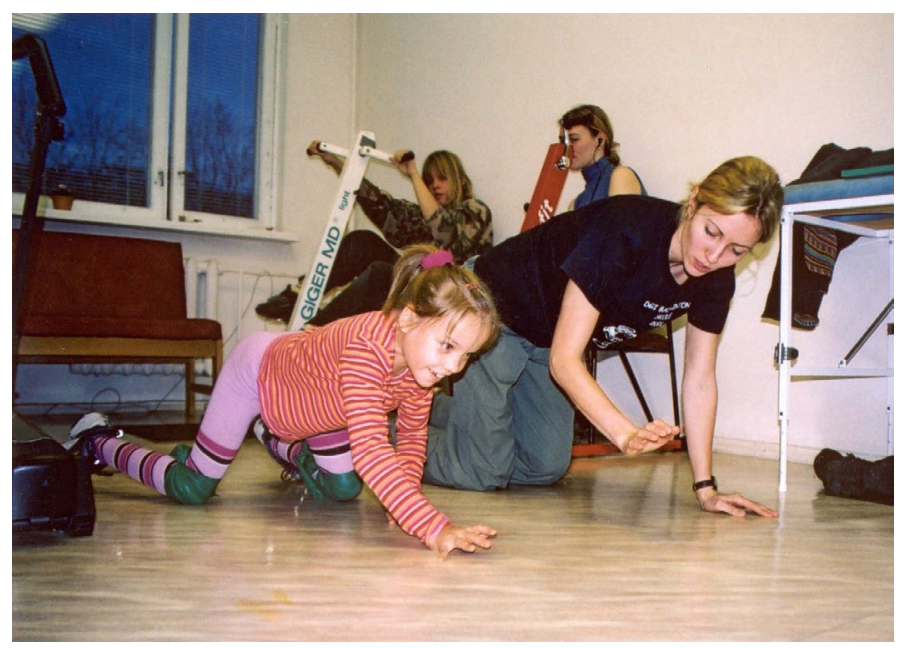

Figure 25. Trot gate crawling of a cerebral palsy girl in interpersonal coordination with the therapist. The crawling performance of the therapist is not optimal. The right arm is leading with respect to the left knee. The crawling performance of the patient is also not optimal; the knees are too much apart 


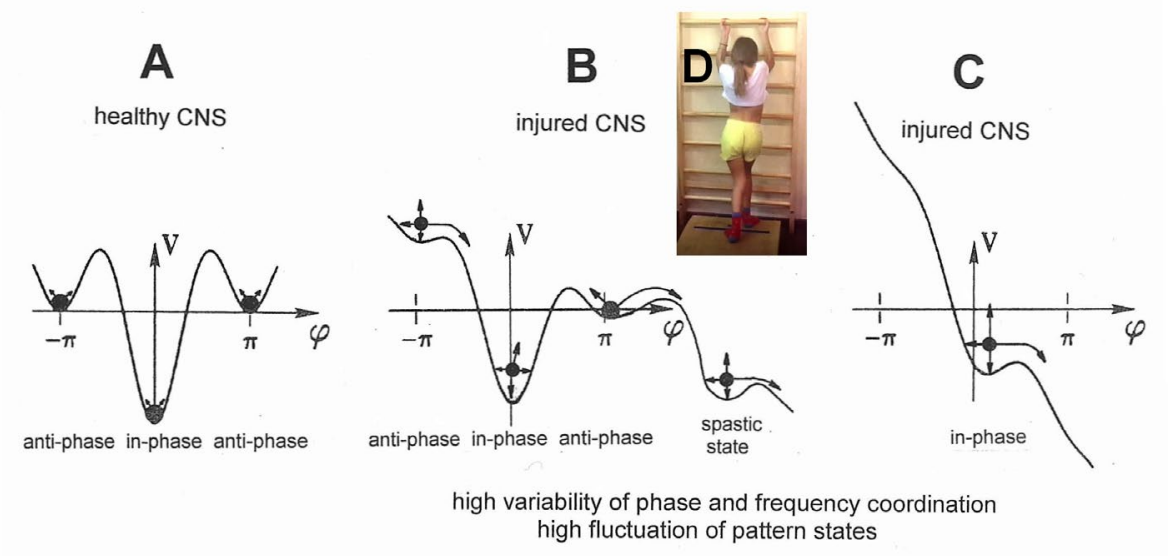

Figure 26. The potential, V( $\varphi$ ), of the coordination dynamics for jumping on springboard (D) of a healthy (A) and injured CNS (B,C). The region around each local minimum act like a well that weakly traps the system into a coordinated state. Behavioral changes are represented by the over-damped movement of a rolling ball in the potential "landscape". High fluctuations (indicated by long arrows attached to the ball (network state)) in the stable state, due to high variability of phase and frequency coordination (in the injured case), will have a greater probability of "kicking" the system out of the basins of attraction (B,C) than for low fluctuations (short arrows) (A), due to small variability of phase and frequency coordination (in A). In $\mathrm{B}$, only the in-phase jumping is stable, even though the fluctuation is high. In $\mathrm{C}$ there is only an attractor basin for the in-phase jumping, but the fluctuation is so high that there is a high probability that the system is kicked out of the basin of attraction. The patient can no longer jump in anti-phase and has difficulty with jumping in-phase. The stability of jumping depends on the motor program (deepness of basin of attraction) and the fluctuation of the pattern state (moving of the ball) caused by the increased variability of phase and frequency coordination due to the injury

the therapy to be updated. With respect to a healthy athlete, the movement performance can be improved by modulating the behavioral information by for example including in the training program the exercising on a special CDT device to improve CNS functioning.

If the behavioral information includes the exercising of extremely coordinated, integrative movements, like exercising on the special CDT device, then the quality of CNS self-organization can be enhanced by improving the exactness of self-organization, namely the precision of phase and frequency coordination between neuron and neural assembly firings. By improving the precision of organization of the intrinsic dynamics, that is the specific variability of the injured networks, certain patterns do eventually re-appear in the case of repairing the injured CNS by movement-based learning.

\section{Learning implications for treatment derived from the equations of} motion of the collective variables

From the repair by learning in the severely injured CNS we learn about learning in the healthy CNS, because the impact on the learning mechanisms is higher than in animal experimentation.

1. Behavioral requirements $\mathbf{F}_{\text {inf }}$ (like intention, support, and instruction) affect the whole coordination dynamics, including stability (see below), rather than only certain coordination patterns. The change of the whole coordination pattern dynamics of the CNS by the behavioral information is one scientific basis for learning transfer [28] between different patterns and stability changes of patterns (as for example the reduction of spasticity). The other scientific basis for learning transfer is followed from human neurophysiology, namely that nerve cells or neural sub-networks are involved in different neural network organizations [4].

2. The intrinsic dynamics $\mathbf{F}_{\text {intr }}$ include vegetative and higher mental functions (these are also patterning of the coordination dynamics), which indicate that via exercising coordinated movements with support and/or instructions $\left(\mathbf{F}_{\text {inf }}\right)$, urinary bladder function, intelligence and speech may be partly repaired or improved following CNS injury or malformation.

3. When in an injured CNS with a certain set of behavioral information $\left(\sum c_{\text {inf }} \mathbf{F}_{\text {inf }}\right)$ the intrinsic coordination dynamics $\left(\mathbf{F}_{\text {intr }}\right)$ can no longer be influenced during coordination dynamics therapy, then this set of behavioral information has to be changed (using different $\mathbf{F}_{\text {inf }}$ ), or balanced differently (using different $\mathbf{c}_{\text {inf }}$ ), to further improve CNS organization dynamics.

4. However, the equations of motion of the coordination pattern dynamics (formula 2) provide no information about the specific behavioral information $\left(\mathbf{F}_{\text {inf }}\right)$ and training intensity $\left(\mathbf{c}_{\text {inf }}\right)$ with which the CNS can be efficiently repaired by learning in a patient. We need to have detailed knowledge of the human CNS at the single neuron and neural assembly level, as well as knowledge at the integrative level, to find the specific behavioral information for the repair by learning of the human CNS.

A first novel step in coordination dynamics therapy is the inference derived from the equation of motion. It suggests that the movement learning not only improves the performance of that particular movement, but also improves the other non-trainable functions by transfer of learning [28,29]. These functions include vegetative functions like bladder control, speech and higher mental functions.

Furthermore, we have means by which the stability of physiological network states can be increased (e.g. movements, continence, continuous concentration in performing certain tasks, speech etc.) and simultaneously the stability of pathological network states, like spasticity, decreased. The coordination (pattern) dynamics therapy partly based on the System Theory of Pattern Formation in combination with human neurophysiology thus offers us an important theoretical basis and a practical tool to diagnose, quantify and repair/improve the functioning human nervous system at the macroscopic level.

Geographical landscape of attractors: The drawback of the equation of motion of the order parameters (formula 2) is that it is normally not possible to find a mathematical solution to it. But by defining a potential function and by picturing the attractive states and attractors by a ball in a potential well or rather by a ball moving in a geographical landscape of attractors (Figure 26), we form a theoretical basis to understand and measure stability of certain coordinated movement patterns (i.e. the deepness of the potential well of an attractor) in patients with CNS injury who receive on-going therapy. 
To make the strategy of pattern formation, pattern stability, pattern assessment, and pattern picturing understandable, the procedure is demonstrated for the simple movement 'jumping on springboard' (Figure 34), which is used during CDT, especially for the repair of the urinary bladder and training in the up-right weight-bearing posture (very important in patients with SCI).

For the special movement 'jumping on springboard' with no behavioral information $\left(\sum \mathrm{c}_{\text {inf }} \mathbf{F}_{\text {inf }}(\mathbf{X}, \mathrm{t})=0\right)$ the equations of motion (formula 2) take the form $\mathrm{d} \varphi / \mathrm{dt}=\mathrm{f}_{\text {intr }}(\varphi)$, where $\varphi$ is the relative phase between the two moving legs and is the only collective variable of this special movement.

The mathematical solution of $\mathrm{d} \varphi / \mathrm{dt}=\mathrm{f}_{\text {intr }}(\varphi)$ in the Haken-KelsoBunz model [30,31] gives the equation of motion for jumping on a springboard for the symmetric case. The obtained potential function $\mathrm{V}(\varphi, \mathrm{t})=-\mathrm{a}(\mathrm{t}) \cos \varphi-\mathrm{b}(\mathrm{t}) \cos 2 \varphi$ can be plotted for different $\varphi$ and certain ratios of the parameters $a$ and $b$ and is shown in Figure 26.

The potential function shows two attractor states, namely the jumping in in-phase $(\varphi=0)$ and the jumping in anti-phase $(\varphi= \pm \pi)$. Especially for higher frequencies (smaller b/a) the jumping in-phase has a higher stability (the potential well is deeper) than the jumping in anti-phase. Asymmetry (not tackled mathematically here) strongly changes the stabilities of the attractor states (depths of potential wells) (Figure 26).

The human CNS, seeking for cooperative stability, slips into the collective states to which it is attracted. For jumping on springboard these attractive states are the jumping in in-phase and in anti-phase. For crawling (not creeping) the attractive states are the pace (inphase) and in trot gait coordination's (anti-phase). When introducing the variability of phase and frequency coordination of strength $Q$, the potential function takes the form $\mathrm{d} \varphi / \mathrm{dt}=-\mathrm{dV}(\varphi) / \mathrm{d} \varphi+\left(\mathrm{Q} \xi_{\mathrm{t}}\right)^{1 / 2}$, where $\xi_{t}$ is Gaussian white noise of unit variance.

The behavioral changes (Figure 26) when jumping on springboard (Figure 34) are represented by the over-damped movement of a rolling ball in the potential landscape for the physiologic (Figure 26A, Q small = little fluctuation of phase and frequency coordination) and the pathologic case (Figure 26B, C; Q large = large variability). The increased fluctuation in the rather stable state, due to increased variability of phase and frequency coordination, will have greater probability of "kicking" the system out of attractor the basin (Figure 26B, C), especially in the asymmetric case.

In the healthy CNS, the phase and frequency variability are small (short arrows) and the jumping in-phase and anti-phase is stable (Figure 26A). Following injury, the potential landscape is deformed and the fluctuation of the network states, generating jumping, is high (Figure 26B). The in-phase jumping is still stable in spite of the increased fluctuation, because the basin of attraction is deep. The jumping antiphase became unstable because the basin of attraction is shallow and the increased fluctuation in the state has a greater probability of "kicking" the system out of the basin. A switch into a spastic state is also possible. In severe CNS injury or malformation, the patient cannot jump any more in anti-phase because of the missing of attractors for anti-phase jumping (Figure 26C). Support is needed for anti-phase jumping. The jumping in-phase is still possible but unstable.

Upon performing very exact coordinated movements, imposed by devices (Figure 1), the nervous system of the patient learns to reduce the variability of phase and frequency coordination and achieves in this way a small fluctuation of the network states again as shown in Figure

26A. The progress in treatment (learning) is that the in-phase jumping in Figure 26C and the anti-phase jumping in Figure 26B become stable again (Figure 26A). Also, the potential landscape will change due to the reduction of the phase and frequency variability. The important consequence for treatment is that when exercising on special CDT devices and reducing in this way the variability of phase and frequency coordination, the patient can induce the formation of patterns again, without having trained them (learning transfer). Upon improving the coordinated firing of neurons, a cerebral palsy child my become able to speak or may develop social behaviors.

In conclusion, the impairment of phase and frequency coordination, measured at the neuron level in human, can be included in the coordination dynamics at the collective variable level. The decrease of the variability of phase and frequency coordination (one kind of coordination repair) is an essential part of CNS development and repair by movement-based learning.

Reduction of spasticity: When performing movements like crawling, walking, running, or exercising on a special CDT device which imposes highly coordinated movements on the patient, the coordination dynamics can be changed in the way that the stability of spastic states decreases, and the stability of the movement states increases. Such changes of coordination pattern dynamics can be pictured again by means of an attractor layout. An attractor is pictured as a potential well (attractor valley) into which a rolling ball is attracted. The position of the ball represents the momentary state of the system. Figure 27 shows schematically such an attractor layout with the two attractor's spasticity and coordinated movement (Figure 27). When exercise is commenced (A), the spastic state is very stable (the attractor valley is deep) and the state of the system is attracted towards the attractor state spasticity. With exercise, the attractor layout is changing in the short-term memory in the way that the attractor spasticity is getting shallower and the attractor physiologic movement is getting deeper (B). Because of fluctuation due to variability of phase and frequency coordination, the position of the ball, which represents the momentary state of the system, is switching between the attractor states spasticity and movement. Spasticity and movements are present simultaneously in the patient. With further exercise, the attractor movement becomes deeper (more stable) than the attractor spasticity. The patient can now perform the movements

Improvement of the coordination dynamics in the short-term memory

A

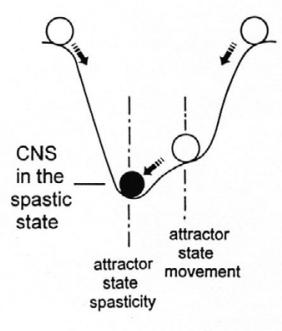

B

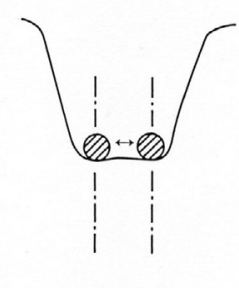

C

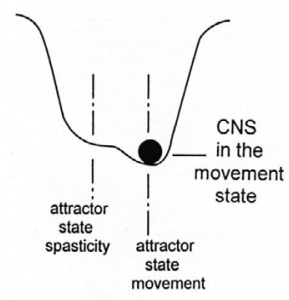

Figure 27. Therapy-induced spasticity reduction in the short-term memory. The position of the ball represents the state of the system and the potential well, the attractor. The ball is attracted to the stable position in the deepness of the hole, called attractor state. The attractor layout, consisting of two attractive states of different stability, is changing upon exercising very coordinated rhythmic movements. Black ball $=$ stable state, open ball $=$ very unstable state, hatched ball = spasticity and movement co-exist. Variability of phase and frequency coordination is not indicated 
with little or no spasticity. The transient reduction of spasticity in the short-term memory, achieved by many hundreds of coordinated movements can last up to several hours; this is indicated in Figure 27 by the two long arrows. The shorter backward arrow (from right to left) indicates that spasticity has slightly reduced in the long-term memory. The coordination dynamics have changed. Repeated exercising will further reduce the stability of spasticity and increase the stability of the coordinated movement and will further change intrinsic coordination tendencies in the long-term memory.

Repair of the stability of the pattern 'running on treadmill': It is not sufficient to re-learn a pattern following CNS injury. It is important that the patient can maintain a given pattern as long as desired. Therefore, pattern stability also has to be repaired. When a patient with a severe brain injury re-learned to run on treadmill, the stability of the pattern for running was repaired and measured. The measure used for stability increase was how long the patient can run without losing the pattern. The longest running period per day was used to quantify repair progress. He could manage his balance problems by briefly touching the rail of the treadmill. However, when he lost the pattern, the treadmill had to be stopped and he had to concentrate for some time before starting again.

Figure 28 shows the improvement of running at $8.5 \mathrm{~km} / \mathrm{h}$ on treadmill. At the beginning he could run only $2 \mathrm{~min}$ before losing the pattern. Eight months later, he could run $30 \mathrm{~min}$. The stability of the running pattern had increased by a factor of 15 (Figure 28).

The increase of running pattern stability of the patient is pictured in Figure 28 by a ball moving in a potential well. For increased running times the potential well is drawn deeper and the arrows, characterizing variation of phase and frequency coordination, are made smaller. The question is now, what contributed more to the increase of running pattern stability, the deepening of the potential well (according to the system theory of pattern formation) or the reduction of phase and frequency variation? The increase of the running stability coincided with a decrease (improvement) of the high-load CD values (therapy year 2012, Figure 30). The improvement of phase and frequency coordination will have therefore contributed to the improvement of the running pattern stability, but the main contribution for stability increase will probably have come from the deepening of the potential well, that is to say from a stronger establishment in the networks of the attractor running on treadmill. The increase of the stability of the pattern running was therefore achieved by both a deepening of the potential well for running and the reduction of the variability of phase and frequency coordination as shown in Figure 26 for jumping on springboard.

Healthy humans may tire during running but they do not lose the running pattern, because the stability of the running pattern is very high. It is as if the running pattern is hard-wired, similar to crawling. This patient also occasionally lost the crawling pattern, even though crawling improved strongly during his therapy. When a tennis player is serving one can see that the pattern stability of serving with good performance can vary significantly.

Quantifying the improvement of CNS functioning by measuring pattern stability upon pattern change when exercising on the special CDT device: Experimentally, the underlying dynamics of coordinated movements can be found in the temporal stability of coordination patterns and can be assessed through pattern change (second novel step). A change of the coordinated movement patterns is generated, when a subject is exercising on the special CDT and recording device (Figure 1), where the coordination between arms and legs, imposed by the device, changes continuously between pace $(\mathrm{P})$ and trot gait $(\mathrm{K})$ and backwards. The stability of the intrinsic coordination pattern tendencies is measured by the deviations and differential stability during the performance of these rhythmic movements. When the differential stability of the movement pattern is high, the arrhythmicity of exercising is small and when the stability is low the arrhythmicity of exercising in that pattern is high. From a standard coordination dynamic trace, it can be seen that in the healthy case the arrhythmicity

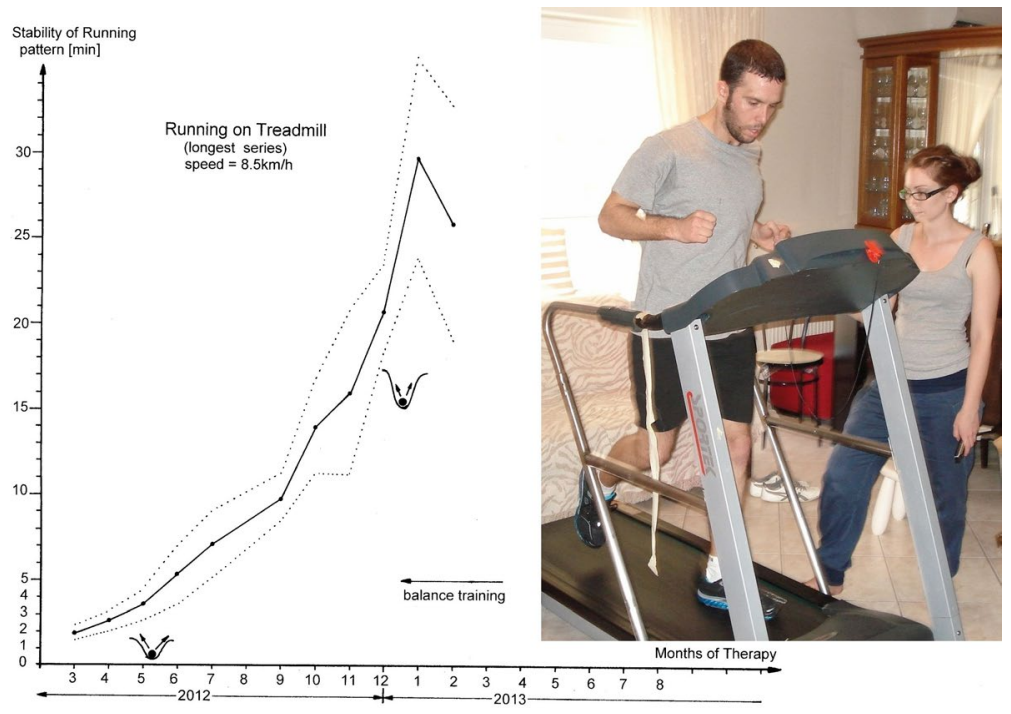

Figure 28. Stability of the movement pattern 'running on treadmill in the forward direction' in dependence on therapy time in a patient with severe brain injury. The stability of the movement pattern 'running' is quantified by the longest time in minutes the patient could stay in the running pattern with several trials and following a warming-up running. Note that the stability improved strongly. Two insets characterize the increase of the 'pattern stability' with a ball (state of the system) in a potential well. This picturing of stability has its scientific basis in the system theory of pattern formation and human neurophysiology. Within the system theory of pattern formation, the stability of a pattern is pictured by a ball (the state of the system) in an attractor layout (here the potential well). The variability of the pattern running (the 'jumping of the ball' in the potential well) is characterized by the lengths of arrows and has its scientific basis in the variability of the phase and frequency coordination of neuron firing (human neurophysiology). Note, with on-going treatment the potential well (the attractor) is getting deeper and the arrows are getting shorter (improvement of phase and frequency coordination) 
is low for the pace and trot gait coordination's and is high for the intermediate coordination's between pace and trot gate in similarity to the recording from a patient with Parkinson's disease (Figure 29B). The pace and trot gait coordination's between arm and leg movements have a high stability (low amplitude arrhythmia) and the intermediate coordination patterns have a low stability (high arrhythmia).

The mean stability per minute can be measured by the arrhythmia of exercising ( $\mathrm{df} / \mathrm{dt}: \mathrm{f}, \mathrm{f}=$ frequency; or $\mathrm{d} v / \mathrm{dt}, v=$ angular velocity). Such differential stability value per minute, the so-called coordination dynamics value, quantifies CNS functioning objectively, integrative and non-invasively. The practical assessment of quality of CNS organization by pattern change is a third novel step in CDT.

Figure 29 shows a high-load coordination dynamics measurement of a treated patient with Parkinson's disease (Figure 29A, B, C) and the trained and fit Author (Figure 29D, E, F) (Figure 29). The Author had very little arrhythmia for low load (D) and also for approximately $150 \mathrm{~N}$ (E, lower trace). The patient had normal arrhythmia for low load (A), but high arrhythmia for $150 \mathrm{~N}$ (high-load). With the shown full highload test, the fit patient and the fit Author showed both load escape. With increasing load, the frequency of turning reduced to escape the load. Such high-load test for increasing and decreasing load is a good measure to follow up the brain repair over years.

Figure 30 shows the brain repair measurements in a patient with a very severe brain injury following a car accident. The patient needed 4 years to reach comparable good high-load coordination dynamics values (Figure 30). For comparing the rate of repair (the improvement curves of the high-load coordination dynamics values) of an athlete (C) and a healthy pupil (B) are inserted. Note that the brain-injured patient needed much more time to achieve similar good $C D$ values. The athlete and the pupil needed for the substantial improvement of brain functioning approximately 1 month. For roughly the same nervous system improvement the patient with the very severe brain injury needed 4 years, that means the efficiency of movement-based learning was lower by approximately a factor of 50 .

Now we are coming back to quantify the brain repair improvement by human electrophysiology.

Reduction of tremor and improvement of the motor program in the short-term memory after exercising on the special CDT device in a patient with Parkinson's disease: Here it is shown that when a patient with Parkinson's disease exercised on the special CDT device, the tremor muscle activity coordinated its firing with the movementinduced motor program and reduced in general during exercise [32]. The tremor activity was therefore brought under the control of the movement activity and was influenced by it to reduce. When increasing the load, also the motor program improved.

When the patient was positioned on the special CDT device, but not exercising on it, rhythmic muscle activity was recorded from the right tibialis anterior muscle. The rhythmic activity occurred at a frequency of $3.4 \mathrm{~Hz}$ and the amplitude was $\approx 200 \mu \mathrm{V}$ (Figure $31 \mathrm{~A}$ ). No activity was recorded from the gastrocnemius, biceps brachii and triceps brachii muscles. When the patient exercised on the device, muscle motor programs were recorded from all of the four above mentioned muscles (Figure 31B). Motor program activity and tremor muscle activity was recorded from the tibialis anterior muscle (Figure 31B). The frequency of the motor program activity during exercise was $\mathrm{f}=0.9 \mathrm{~Hz}$ and the frequency of rhythmic tremor activity was now $1.7 \mathrm{~Hz}$. The tremor activity reduced from $3.4 \mathrm{~Hz}$ (Figure $31 \mathrm{~A}$ ) to $1.7 \mathrm{~Hz}$ (Figure $31 \mathrm{~B})$ and coordinated its firing with those of the motor program. The tremor amplitude reduced from $200 \mu \mathrm{V}$ to $100 \mu \mathrm{V}$ and the duration of the tremor activity bursts became shorter.

In another recording set from the same patient (Figure 31D) it seemed as if the tremor reduction was more pronounced for higher $(20 \mathrm{~N})$ than for lower load exercise $(10 \mathrm{~N})$; this is to be expected since exercising at higher load will activate the CNS more integrative, so that the influence of the physiologic CNS organization upon the pathologic organization will be larger (Figure 31 ). With ongoing exercising on the device tremor reduction could nicely be seen from the reduction of tremor arm movements. At the beginning of exercising, the arm tremor amplitude was large and afterwards reduced. The patient had tremor in arms, legs and face on both sides of the body. Visible tremor reduction need not necessarily mean however that the rhythmic tremor muscle activity had reduced, since the tremor amplitude could have been influenced by movement kinetics. It was therefore important to show through sEMG that the muscle activity leading to tremor really had reduced.

The reduction of tremor muscle activity means that less upstream motoneurons synchronized their firing in the short-term memory. The coordinated firings of neurons had improved in the short-term memory. With repeated training on the special CDT device, the improved phase and frequency coordination will be established in the long-term memory.

In another measurement, the tremor muscle activity reduced in the recorded sweep piece; tremor reduced from $5 \mathrm{~Hz}$ to $1.8 \mathrm{~Hz}$.

In two patients with Parkinson's disease the motor program improved after a few minutes of exercising on the device (Figure 31). Figure $31 \mathrm{C}$ shows that the motor program improved in the tibialis anterior muscle during that sweep piece when exercising at a load of 10 N. From Figure 31E, F it becomes evident that the motor program improved substantially in the tibialis anterior muscle when increasing the load from $50 \mathrm{~N}$ to $150 \mathrm{~N}$. Therefore, the motor program improved with ongoing exercise and with the increasing load provided that the patients could manage the load.

Treatment of locomotion judgment according to surface electromyography (sEMG) for improving repair:

To optimally entrain severely damaged neuronal networks, integrative movements which can be performed by a patient most physiologically, can be quantified by surface electromyography (sEMG) and used then, if wanted, most frequently. Such surface EMG diagnostic is shown in (Figure 32).

Figure 32 shows the motor programs of the left brachioradialis, extensor carpi radialis, triceps surae and tibialis anterior muscles during walking (A), running on treadmill (B), jumping on springboard (C), and exercising on a special coordination dynamics therapy device (D). It can be seen from Figure 32 that the motor programs of the four muscles for running and jumping on the springboard $(B, C)$ were better than those for walking (A) and exercising on the special device (D). In this patient, running and jumping on the springboard thus seemed to be more efficient in respect of CNS repair than walking and exercising on the special CDT device. Improvements of CNS functioning were quantified clinically, largely by improvement in the performance of movements. This patient Benjamin could walk and run fast after one year of treatment. Only the performance of the left arm and left leg were poor in nearly all movements. The writing performance was poor, and his intellectual functions had not fully recovered. 


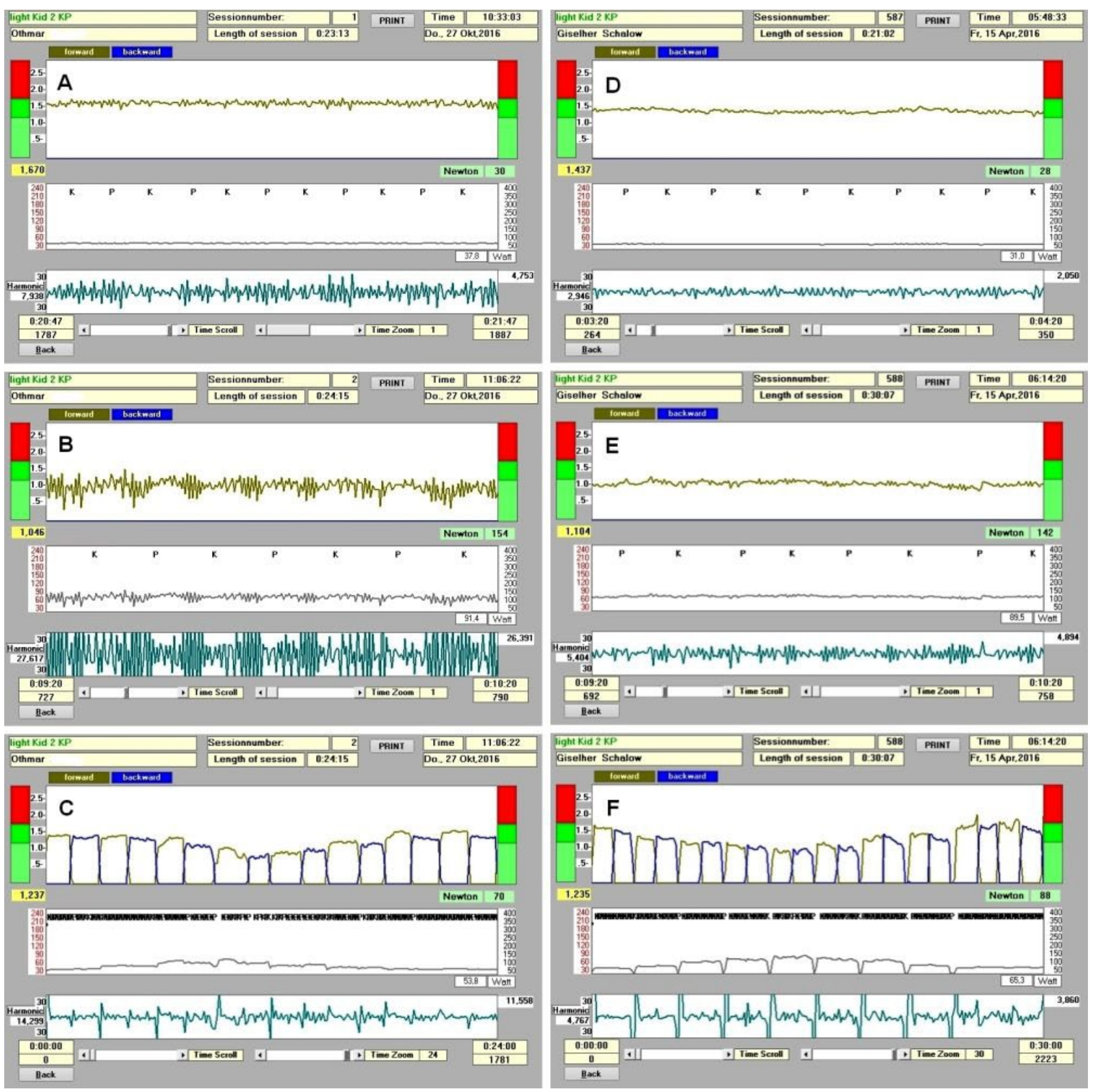

Figure 29. Coordination dynamics (CD) measurements of a 70-year-old patient with Parkinson's disease (A,B,C) in comparison to ones of the Author with a very good functioning CNS $(\mathrm{D}, \mathrm{E}, \mathrm{F}$; similar age). Upper trace $=$ frequency, lowest trace $=\mathrm{CD}$. Note that the arrhythmia of exercising $(\mathrm{CD})$ in the patient is much larger $($ worse) for $30 \mathrm{~N}(\mathrm{~A})$ and $150 \mathrm{~N}(\mathrm{~B})$ than for the Author (D,E); for $30 \mathrm{~N}$ by $\approx 250 \%$ (4.8 against 2 ) and for $150 \mathrm{~N}$ by $400 \%$ (26 against 4.9$)$. For the high-load test (C,F) a nice stepwise increase and decrease of the work (Watt) with increasing and deceasing Newton can be seen for the Author but not for the patient. The patient could only reach $150 \mathrm{~N}$, the Author $200 \mathrm{~N}$. The patient was performing coordination dynamics therapy for 3 years, the Author for 9 years. Note in B that the patient had problems with the intermediate coordination's between pace (P) and trot gait (K) for 150N (higher arrhythmicity); the Author not 

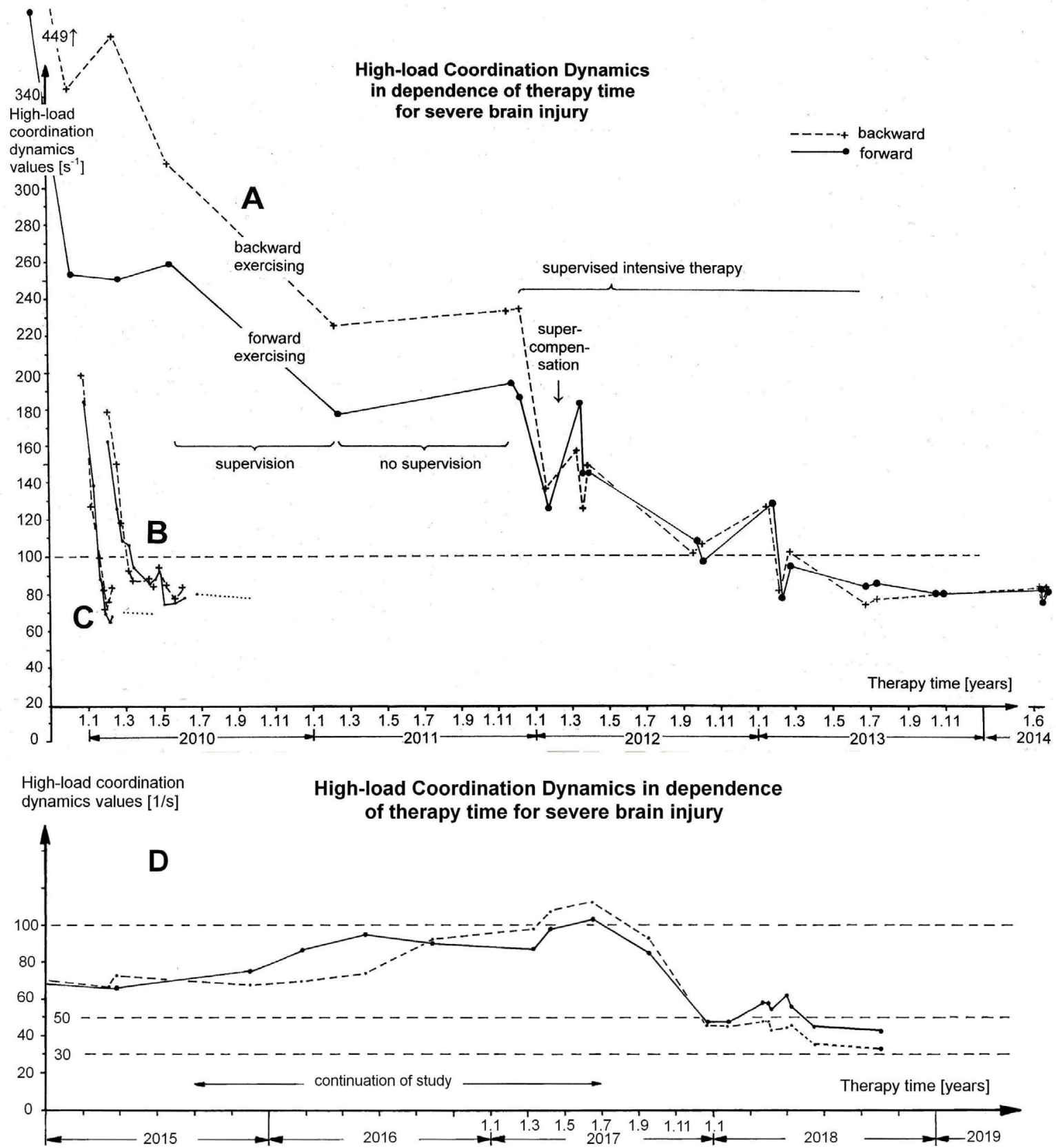

Figure 30. A. Improvement of high-load coordination dynamics (CD) values in a patient with severe brain injury (same patient as in Figure 28) upon coordination dynamics therapy for several years. The high-load CD values were obtained by summing up the single CD values for forward and backward exercising, $\Delta$ (high-load $\mathrm{CD}$ value $)=\Delta 20 \mathrm{~N}+\Delta 50 \mathrm{~N}+100 \mathrm{~N}+\Delta 150 \mathrm{~N}+$ $200 \mathrm{~N}+\Delta 150 \mathrm{~N}+\Delta 100 \mathrm{~N}+\Delta 100 \mathrm{~N}+\Delta 50 \mathrm{~N}+\Delta 20 \mathrm{~N}+\Delta 20 \mathrm{~N})$. B, C. For comparing the rate of repair, the improvement curves of the high-load coordination dynamics values of an athlete (C) and a healthy pupil (B) are inserted. Note that the brain-injured patient needed much more time to achieve similar good CD values. D. Continuation of therapy. The substantial improvement of the high-load CD values (lower values), in the middle of 2018, motivated Sotiris to continue his therapy at the limit

Repair of urinary bladder functions - continents: The repair of urinary bladder functions, and especially the continence, is of high importance for patients with spinal cord injury, multiple sclerosis or cerebral palsy and for women and in ageing in general.

Clinical urinary bladder function test (Urodynamics): To understand learning transfer, defined within the framework of System Theory of Pattern Formation for Repair, from movements to urinary bladder functions with human electrophysiology at the single-neuron level, it is started with the clinical diagnostic of the urinary bladder; urodynamics. The functioning of the urinary bladder can be evaluated by measuring the pressure in the bladder and in the abdomen (colon) and the electromyographic (EMG) activity of the external sphincters and/or functionally associated pelvic floor muscles [45]. Such bladder diagnosis is called urodynamics (Figure 33). Especially the simultaneous activation of the detrusor (detrusor pressure in Figure 33B) and the sphincteric and/or pelvic floor muscles (pelvic floor EMG in Figure 33B), the so-called detrusor-sphincter-dyssynergia, can be measured, which may destroy the kidneys on the long term. 


\section{Improvement of motor program and reduction of tremor in Parkinson's disease patients}

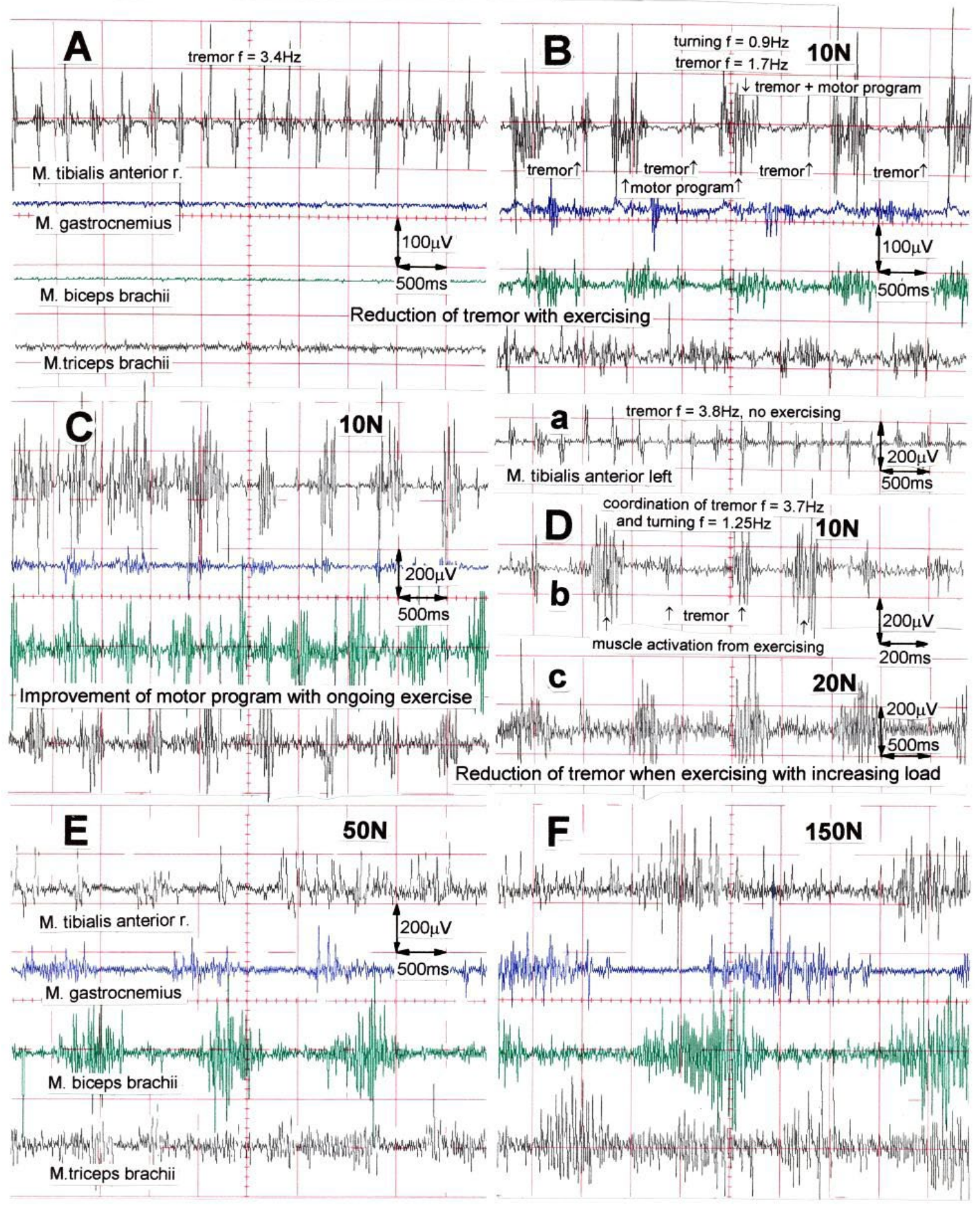

Figure 31. Improvement of the motor program and reduction of tremor in 3 Parkinson's disease patients upon exercising on the special coordination dynamics therapy device. A. Patient is in position at the special coordination dynamics therapy device, but not exercising. Non-voluntary rhythmic muscle activation with $\mathrm{f}=3.4 \mathrm{~Hz}$ is recorded from the tibialis anterior muscle of the right side. B. When exercising on the device at $10 \mathrm{~N}$, a motor program appears in all muscles recorded from. The tremor activation in the tibialis anterior muscle reduced in amount and frequency (to $1.7 \mathrm{~Hz}$ ), and the tremor muscle activation synchronized with the motor program. C. Improvement of motor program in the tibialis anterior muscle with ongoing exercising. D. Reduction of tremor in the tibialis anterior muscle with the increasing load when exercising on the special coordination dynamics recording and therapy device: a. no exercising, tremor muscle activation full present. b. Tremor reduced in amount and frequency (from 3.8 to $1.25 \mathrm{~Hz}$ ) when exercising at $10 \mathrm{~N}$. c. No strong tremor muscle activation visible at $20 \mathrm{~N}$. E,F. Improvement of the motor program in the tibialis anterior and triceps brachii muscles upon exercising on the special coordination dynamics therapy device at increasing load from 50 to 150 N. A,B,D, a 70-year-old female Parkinson's disease patient (U.H.), C, a 76-year-old female Parkinson's disease patient (I.K.), and E,F, from a 70-year-old male Parkinson's disease patient (V.H.) 


\section{EMG Motor programs}
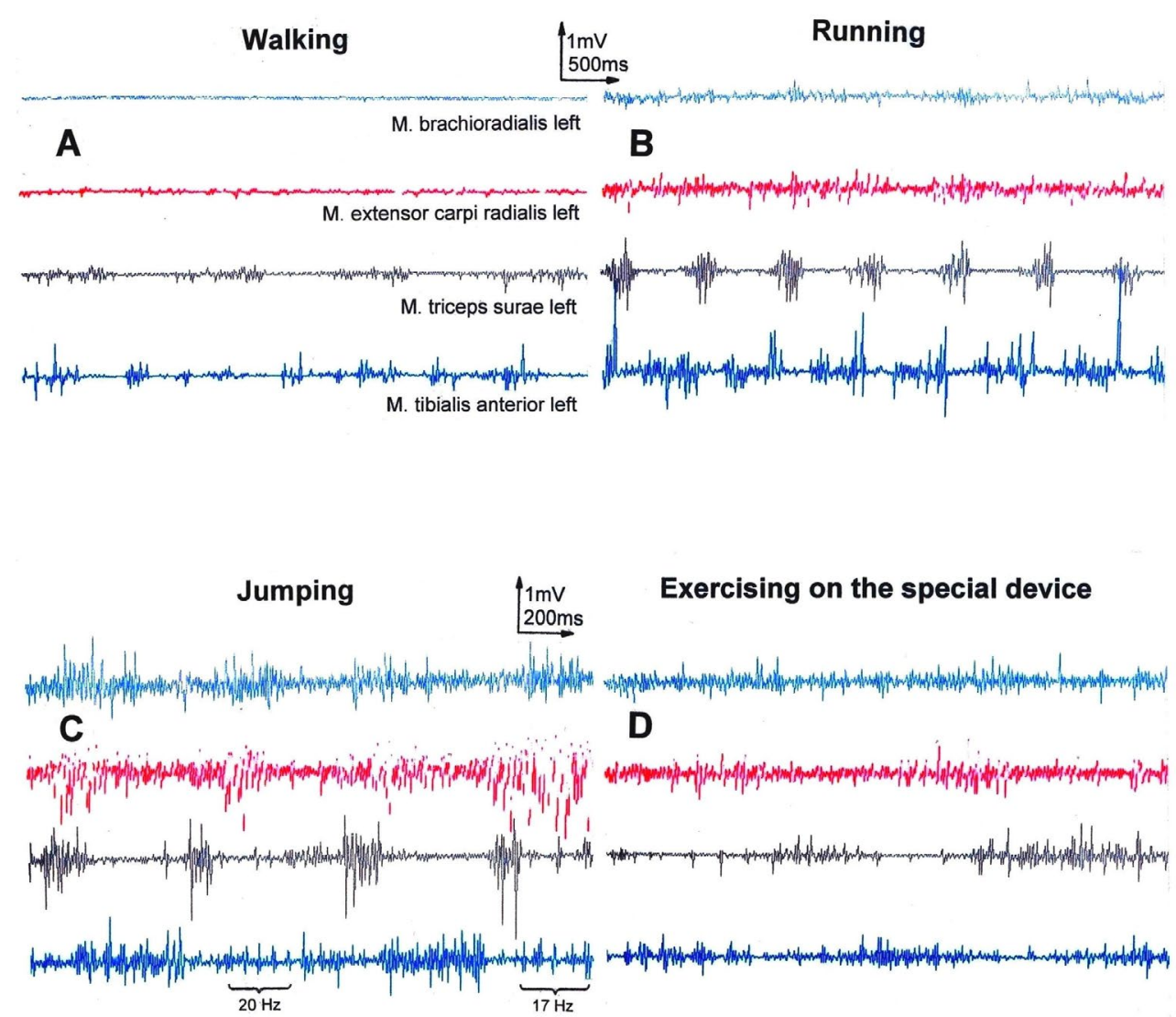

Figure 32. Motor programs recorded by surface electromyography (sEMG) during walking (A), running (B), jumping (C), and exercising on the special coordination dynamics therapy and recording device at the load of $100 \mathrm{~N}$ (D); a patient with formally very severe brain injury. Note that the critical muscles brachioradialis left and extensor carpi radialis left are not activated during walking (A). On the extensor carpi radialis muscle trace, there may be some artifacts on top of the motor program. On the tibialis anterior trace, rhythmic firing of 20 and $17 \mathrm{~Hz}$ can be seen, which most likely stems from FF-type motor units innervated by $\alpha_{1}$-motoneurons

Upon retrograde filling of the bladder in a patient with a spinal cord injury, the pressure in the bladder and colon is measured and the electromyographic activity (EMG) of the pelvic floor is recorded with surface electrodes (Figure 33). The detrusor pressure is obtained by subtracting the abdominal pressure from the bladder pressure. The continence status of the patient is diagnosed by the reports of the first feeling of bladder fullness, the desire to void, and the leaving of fluid out of the bladder. The EMG activity of the pelvic floor informs when the external bladder and anal sphincters are activated. Upon knocking, pressing, coughing, and stimulating bladder reflexes, the bladder status is obtained. This patient of Figure 33 with a spinal cord injury sub Th12 had a detrusor-sphincteric dyssynergia of the bladder, because the EMG activity of the external sphincters increased strongly (the sphincters became activated) with the strong increase of the detrusor pressure (activation of the detrusor for emptying the bladder). Improvement of urinary bladder function mainly due to therapy could be quantified by repeated urodynamics measurements (Figure 33A, B).

Limitation of urodynamics and need for human electrophysiology for causal repair: The evaluation of bladder functioning by means of urodynamics gave the information that the patient had a dyssynergia of the bladder. Repeated testing informed about changes in bladder functioning. But such bladder function tests are giving no information on the pathology of the CNS organization and how to repair urinary bladder functioning causally.

By performing similar bladder tests under operational conditions and recording single-nerve fibre action potentials from sacral nerve roots (Figure 6), the pathology of bladder functioning can be explored $[14,33,34]$ and ideas found how to repair the bladder causally. By exercising on the special CDT device (Figure 1) and jumping (Figure 34 ), the somatic and the autonomic nervous system divisions can be re-organized, and the bladder repaired by learning transfer.

Bladder functioning at the neuron level: By recording from sacral nerve roots (Figure 6) one records also from the urinary bladder (Figure 35).

With the single-nerve fiber action potential recording method it has so far been possible to record natural impulse patterns from parasympathetic efferents (par), urinary bladder stretch and tension receptor afferents $(\mathrm{S} 1, \mathrm{ST})$, mucosa afferents from mechanoreceptors of the bladder, the urethra and the anal canal (M), from afferents responding to fluid movement (S2), and from $\alpha \alpha_{2}, \alpha \alpha_{3}$ and $\gamma \gamma$ motoneurons and muscle spindle afferents innervating the external striated urinary bladder and anal sphincters (or functionally associated pelvic floor muscles), and to analyze regulatory and organizational 

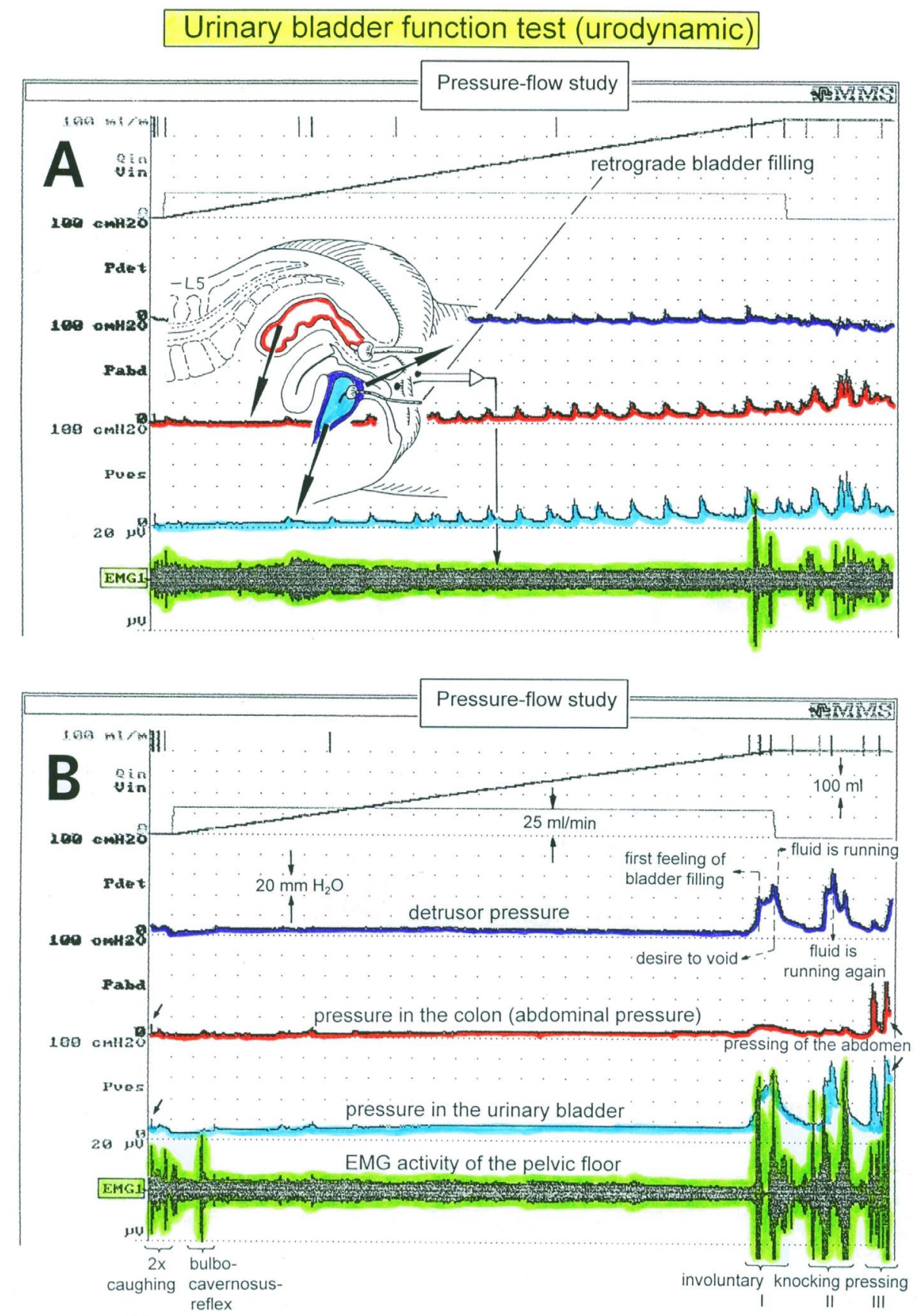

Figure 33. Clinical urinary bladder function test (Urodynamics). Improvement of the urinary bladder functions, quantified by urodynamics in a 30 -year-old female patient. A. 3 months after the accident resulting paraplegia sub Th12 following spinal cord lesion. B. 12 months after the accident (lesion level lowered to sub L3). In A, the detrusor pressure ( $\mathrm{P}$ ) is generated by the contracture of the bladder wall, as the pressure difference between abdominal pressure $\left(\mathrm{P}_{\text {abd }}\right.$, measured in the rectum) and the bladder pressure $\left(\mathrm{P}_{\mathrm{ves}}\right.$, measured in the bladder). Electromyographic recording obtained with surface electrodes from the sphincters and the pelvic floor (EMG) is shown; the external sphincters and the functionally correlated pelvic floor muscles show similar sEMG activity (the rhythmic pressure peaks in A do not originate in the bladder). In A, the detrusor shows nearly no activity with retrograde bladder filling at $25 \mathrm{ml}$ min; in B, the detrusor shows first activity at $360 \mathrm{ml}$ bladder filling. A detrusor-sphincteric-dyssynergia occurs, because the detrusor pressure peaks occur at the same time as the sphincter EMG activity peaks (B) (bladder and sphincter contract at the same time, so that fluid can only emerge from the bladder at high bladder pressure; there is a danger of reflux through the ureter into the kidneys). The EMG peaks are a bit irregular, probably because the fluid, leaving the bladder, shunts transiently the EMG electrodes. Exact functional description of B: $2 \mathrm{x}$ coughing (B below) increases the EMG activity and passively the pressure in the abdomen and in the bladder (marked by the small arrows, physiologic). The bulbocavernosus reflex (induced by pressure applied to the clitoris) increased the EMG activity of the sphincters (physiologic). Conclusion: The reflex arch is in order; sacral nerve roots and nerves have not been damaged in the accident. I (bottom right): The patient feels an increase of unvolitional detrusor pressure (first feeling of bladder pressure at $360 \mathrm{ml}$ ). She tries to contract the sphincters to stop the bladder emptying. Shortly after the desire to empty the bladder, as the detrusor pressure decreases, fluid is leaving the bladder. II: Due to tapping onto the bladder, the bladder reflex is activated (detrusor activated, nearly no abdominal pressure); fluid is leaving the bladder. III: Due to the abdominal muscular pressure the pressure in the abdomen increases as does passively the pressure in the bladder (the detrusor is not activated); fluid left the bladder. With a delay, the detrusor was activated by the bladder reflex. - The urinary bladder of the patient is partly functioning. It has to be further improved by therapy induced reorganization of the CNS: (1) An earlier feeling of bladder filling, (2) an increase of the time difference between the feeling of the first bladder filling and the unvolitionally emptying of the bladder (for the time being, approx. 10 min, in dependence on whether the patient is physically active (such as walking) or not, (3) further learning how to activate the detrusor on volition, and (4) the physiologic coordination between the bladder and the external sphincter functioning (to stop the detrusor-sphincter-dyssynergia). 
mechanisms of parasympathetic neurons and motoneurons in the human CNS.

Upon retrograde bladder filling (as in the clinical diagnostic, Figure 33) and the identification of the neuron type, with the use of the classification scheme for human peripheral nerve fibers (Figure 8), the natural impulse patterns of identified afferent and efferent fibers were obtained and analyzed (see above). The obtained natural impulse patterns answered an old question: Is it the firing rate of a neuron that codes the information transmitted and processed or does the precise timing of cell discharge codes information? Figure 12B,D shows that the information is coded by specific impulse patterns, including the precise timing and the firing rate.

Important for the application of human neurophysiology to neurotherapy of the bladder is the duality of the functions of the sphincteric motoneurons and secondary muscle spindle afferents, subserving somatosensory and autonomic (parasympathetic) functions. Motoneurons innervating the external sphincters of the bladder and the anal canal sub-serve somatic functions (contraction of the sphincters on volition or for protection reaction) and parasympathetic functions for the coordination of the detrusor function (parasympathetic) and the external sphincter function (somatic). The repair of the urinary bladder functioning by reorganization of networks seems to be most difficult because there is false neuronal network organization in the parasympathetic nervous system (overactive (spastic) detrusor) and the somatic division (spastic external (striated) bladder sphincter), and there is false interaction of the interlacing somatic and parasympathetic networks (detrusor-sphincteric dyssynergia: when the detrusor contracts, the external sphincter is also contracting instead of opening (relaxing)) [14,33,34].

Time course comparison between the proposed parasympathetically induced muscle spindle afferent activity and the detrusor pressure: In detrusor-sphincter dyssynergia of the urinary bladder, the somatic external bladder sphincter is activated at the same time as the detrusor (smooth muscle) with the consequence that the bladder cannot be emptied. This dys-coordination between the somatic and parasympathetic nervous system divisions in the human sacral micturition center is reflected urodynamically (clinically) in the simultaneous increase of the detrusor pressure and the electromyographic activity of the pelvic floor. Before analyzing the detrusor-sphincter dyssynergia at the neuron level an important correlation between muscle spindle activity and detrusor pressure,

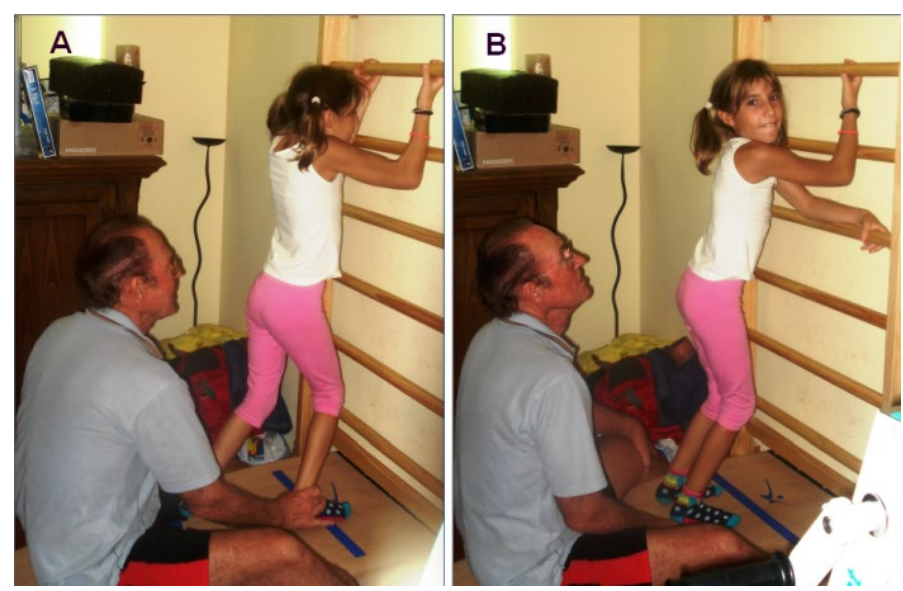

Figure 34. Ten-year-old patient Nefeli with an incomplete spinal cord injury during jumping on springboard, supported by the Author [4] measured urodynamically, has to be done, namely that muscle spindles, also driven by the autonomic nervous system, show very similar activity changes than the detrusor pressure.

It was shown that the activity in parasympathetic efferents can be measured, identified and distinguished from the activity of $\gamma$-motoneurons in conduction velocity distribution histograms (Figure 10). Since the action potential amplitudes of parasympathetic efferents is small, it would still be very difficult to analyze the organization of the parasympathetic nervous system and it's coordinated functioning with the somatic nervous system as in the control of the urinary bladder. But some secondary muscle spindles in the parasympathetic range are also innervated by parasympathetic efferents besides somatic efferents ( $\gamma$-motoneurons). In animals it was found that also sympathetic fibres innervate muscle spindles [35]. The activation of the parasympathetic nervous system can therefore also be measured by the activity of secondary muscle spindle afferents. Since the action potentials of secondary spindle afferents are comparably large (thick fibers), the activation of the parasympathetic nervous system can be easily indirectly assessed. It will be shown now that at least some secondary muscle spindles in the parasympathetic range are innervated by parasympathetic efferents (parasympathetic fusimotors, with the above reassignment sympathetic fusimotors). The evidence is obtained by measuring the parasympathetic activation of the detrusor by detrusor pressure changes and by measuring activity changes of secondary muscle spindle afferent fibers and compare the form of the changes of detrusor pressure with the activity changes of a secondary muscle spindle afferent fiber.

In Figure 36, the increase in detrusor pressure upon retrograde bladder filling before surgery is compared with the activity increase of the secondary muscle spindle afferent fiber SP2(1) during the operation following 4 times bladder catheter pulling. Figure 36A shows the undulating activity increase of a SP2(1) fiber. In Figure 36C the cystogram is shown. Upon bladder filling spontaneous micturition occurred several times (Figure 36). If parasympathetic fibers really activated muscle spindles, then the activity increase of the secondary muscle spindle afferent fibers following bladder catheter pulling may have a similar time course as does the bladder pressure increase due to detrusor activation following retrograde bladder filling. To check this similarity of time course, one undulating increase of detrusor pressure (Figure 36C) has been brought to the same time scale as the measured changes in muscle spindle afferent activity (Figure 36A) and transferred into Figure 36B for a direct comparison with Figure 36A. By comparing Figure 36A with Figure 36B, it can be seen that the occurrence of activity peaks of secondary spindle afferents is very similar in its time course to that of the peaks of the detrusor pressure. From the similarity of changes of spindle afferent activity and detrusor pressure (4 peaks) it can be concluded that some muscle spindles in the domain of the sacral parasympathetic nucleus are partly controlled by the parasympathetic division and that the muscle spindle and the detrusor activation have similar time courses.

Thus, there is indication that some muscle spindles are partly driven by the parasympathetic division. The drive can be by parasympathetic fusimotor activity or more indirectly by somatic fusimotors. Since in HT6, the somatic fusimotors did not change their activity levels strongly with the activation of preganglionic parasympathetic fibres and the activity increase of the secondary muscle spindle afferent fibre SP2(2) followed the transient increase of parasympathetic activity, it is likely that some muscle spindles are directly controlled by parasympathetic fusimotors. For further details see Chapter V of [3]. 


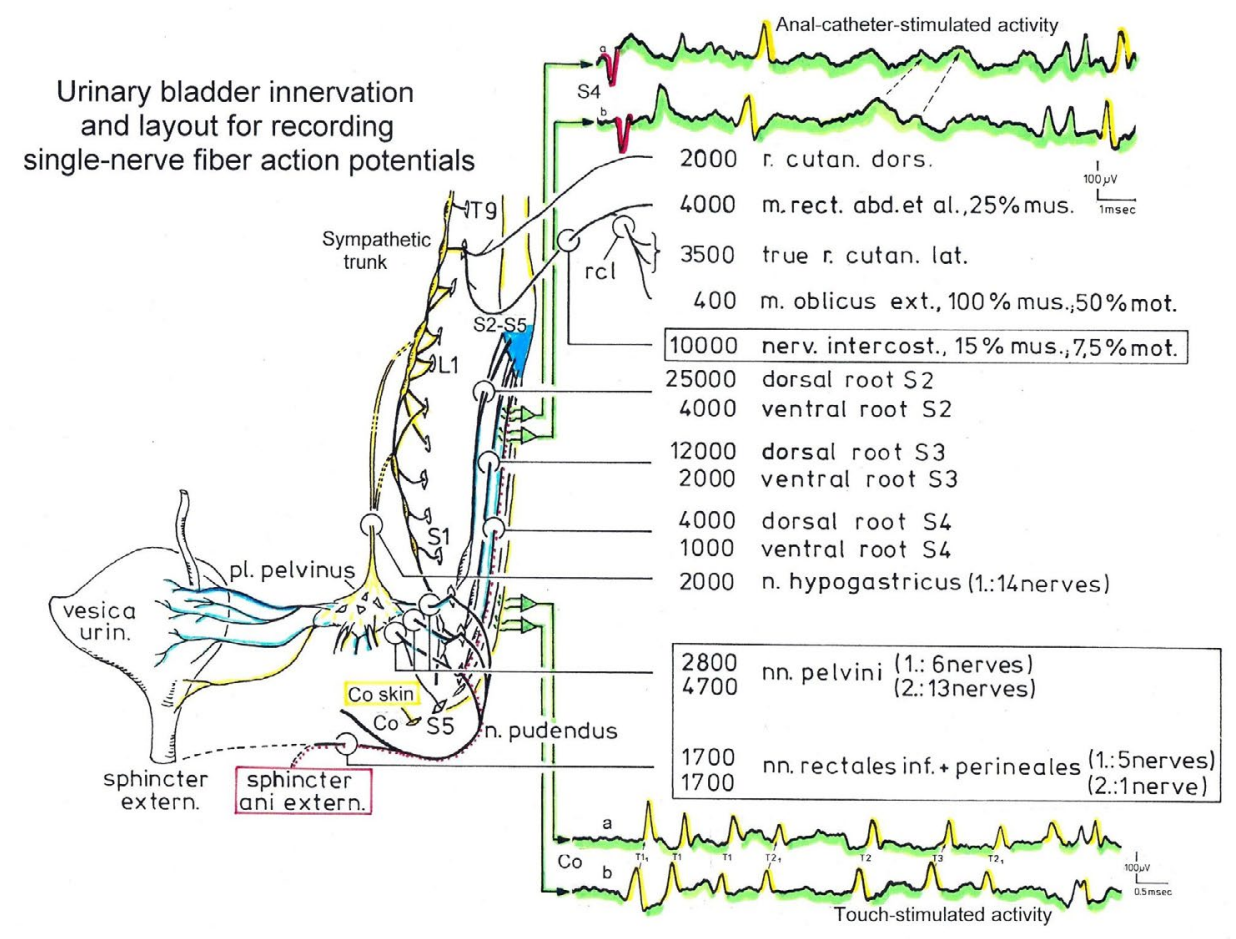

Figure 35. Urinary bladder innervation (anatomy; structure) and recordings of single-nerve fiber action potentials from a S4 and a coccygeal root. Numbers of myelinated nerve fibers of the nervi rectales inferiores and perinales, the nervi pelvini and the nervus hypogastricus are given. $\mathrm{T}=$ thoracal; $\mathrm{L}=$ lumbal; $\mathrm{S}=\mathrm{Sacral} ; \mathrm{rcl}=\mathrm{ramus}$ cutaneus lateralis, no pure skin nerve; \%mus $=\%$ of nerve fibers leading to muscles; $\%$ mot $=\%$ of nerve fibers which are motoric; 1 and $2=$ Nerve fiber counts from cadavers 1 and 2. Numbers of nerves in bracket give the number sub-nerves of which the nerve consisted. The innervation pathway of the external bladder sphincter is unclear. The S4 root recording informs about single-nerve fiber activity running into the spinal cord (from the bladder receptors (Figure 17)) and running out of the cord to the urinary bladder (bladder efferents) upon natural stimulation (touch, pin-prick, bladder and anal catheter pulling (Figures 7,9). Functions of the urinary bladder and the sacral micturition center in the spinal cord, as a part of the human CNS, can therefore be analyzed. The Co root (no efferents; something like a skin nerve) recording informs about skin receptor activity in the coccygeal dermatome

\section{Detrusor-sphincter synergy of the bladder in the brain-dead human HT6, and dys-synergy in paraplegic 9:}

The measurement of parasympathetic activation of the detrusor by activity changes of secondary muscle spindle afferent fibers (the spindle is innervated by autonomic fusimotors) allows an analysis of detrusorsphincter dys-synergy using the natural simultaneous impulse patterns of secondary muscle spindle afferents and sphincter $\alpha_{2}$-motoneurons (and $\gamma$-motoneurons).

Figure 37A shows that in the brain-dead human HT6, whose parasympathetic preganglionic neurons increased activity (Figure $37 \mathrm{Ab}$ ) upon bladder catheter pulling, the SP2(2) fiber activity increased strongly (Figure 37Aa), whereas the $\alpha_{2}$-motoneuron innervating the external anal sphincter discontinued its oscillatory firing (Figure 37Ac), which is a measure for a strong activity decrease. An $\alpha_{2}$-motoneuron, innervating the external (striated) bladder sphincter, was not activated. This means that with the activation of the detrusor the sphincter motoneurons were relaxed by inhibition. Thus, the brain-dead human HT6 had a detrusor-sphincter synergy of the bladder.

In paraplegic 9 who showed a strong activity increase of the SP2(1) fiber, there was no sphincter relaxation following bladder catheter pulling (Figure 37B). The secondary muscle spindle afferent fiber SP2(1) increased its activity in an undulating manner (Figure 37Ba). The parasympathetic fusimotors, driving the muscle spindle, innervated by the SP2(1) fiber, probably were not continuously active as suggested by Figure 37Bb, in contrast to the parasympathetic activity observed in HT6 (Figure 37Ab). The other secondary muscle spindle afferent fiber in paraplegic 9 SP2(2), (Figure 37Ba) slowly reduced its activity upon bladder catheter pulling. This spindle afferent fiber was not connected to the continence of the bladder. It is likely that its spindle was not parasympathetically innervated and was sited in leg muscles or parts of the pelvic floor muscles not contributing to continence. The $\alpha_{2}$ and $\alpha_{3}$-motoneurons (Figure $37 \mathrm{Bc}$ ) showed a high activation, which is expressed in their oscillatory firing, and probably contributed to the continence of the bladder and the rectum. These likely sphincter motoneurons did not reduce their activity following parasympathetic activation, as can be seen from the SP2(1) fiber activity (monitoring parasympathetic activity). These motoneurons were not inhibited and the external sphincters were probably not relaxed. The somatic fusimotor $\gamma_{1}$ (Figure $37 \mathrm{Bc}$ ) increased its activity transiently and slightly upon painful bladder catheter pulling, in similarity to a fiber in HT6 [23]. The measurements in paraplegic 9 indicate a loss of the inhibitory action of the detrusor onto the sphincter motoneurons (Figure 37).

The time constant for the activity decrease of a spindle afferent fiber following parasympathetic activation was $31 \mathrm{~s}$ in a paraplegic and approx. 40s in a brain-dead human (Chapter V) [3]. It is concluded that the muscle spindles are unchanged following spinal cord injury. The pathologic firing patterns of the SP2 fibers are thus probably a result of neuronal network changes in the parasympathetic and somatic nervous system divisions of the sacral micturition center.

In conclusion, in a brain-dead human (HT6) the sphincter motoneurons sub-serving continence were inhibited at a time, when preganglionic parasympathetic efferents increased their activity (physiologic) for 10s and an SP2 fiber increased its activity for several 


\section{Parasympathetic muscle spindle activation in comparison to urinary bladder pressure}

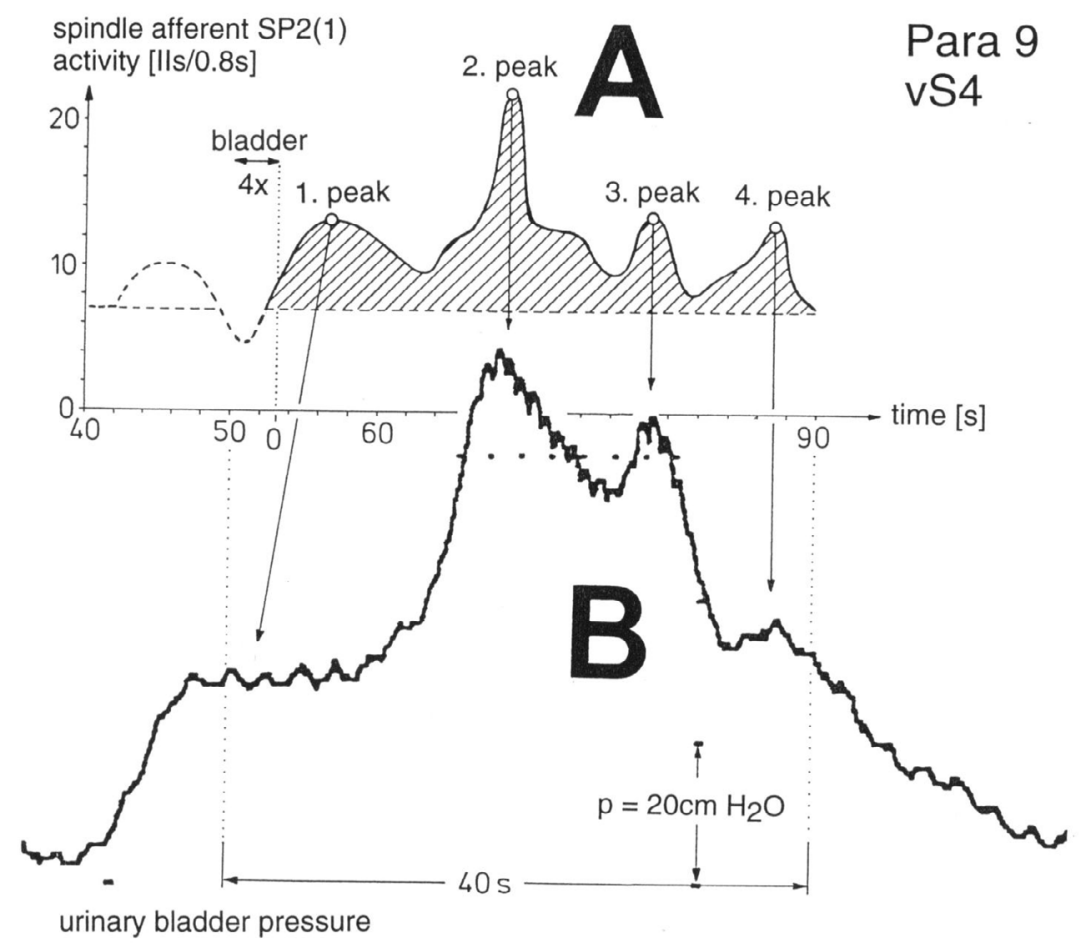

$90 \mathrm{ml}$ filling volume, filling speed $13 \mathrm{ml} / \mathrm{min}$

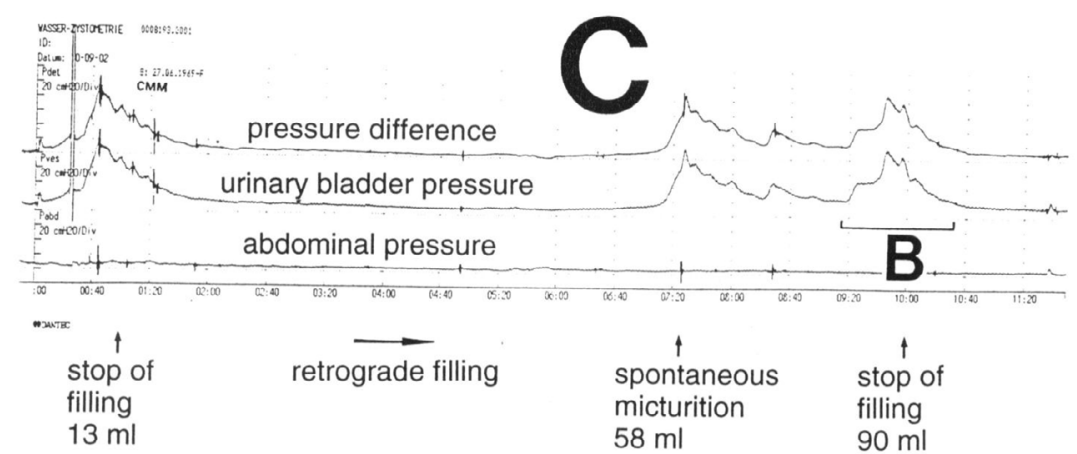

Figure 36. Comparison between changes in secondary muscle spindle afferent activity and detrusor pressure (measured before the surgery; for the implantation of a sacral anterior root stimulator). Paraplegic 9

A. Activity changes of the afferent fiber SP2(1) following bladder catheter pulling (Figure 37Ba). Approx. mean activity level is represented by a dashed line at $7.5 \mathrm{IIs} / 0.8 \mathrm{~s}((\mathrm{APs}-1) / 0.8 \mathrm{~s})$. The activity above the mean is cross-hatched and is proposed to be due to parasympathetic activation. Root vS4.

B. Detrusor pressure (pressure difference) changes taken from ' $\mathrm{C}$ '. Note that changes in the detrusor pressure show almost exactly the same time course as do the activated changes of the secondary muscle spindle afferent fiber SP2(1) of 'A'. Corresponding peaks are correlated by arrows.

C. Abdominal pressure (measured as rectal pressure), intravesical pressure (urinary bladder pressure) and detrusor pressure (pressure difference) during retrograde filling before the surgery. One transient detrusor pressure increase, marked ' $\mathrm{B}$ ', is used, after enlargement in ' $\mathrm{B}$ ', to compare with the spindle afferent activity

minutes. In a paraplegic with a strong bladder dysfunction, the SP2 fiber activity increased, due to parasympathetic activation, lasted for approx. one minute, showed undulations, and its amplitude was smaller than that measured in a brain-dead human. The sphincter motoneurons were not inhibited (pathologic).

In animals the nervous control of the urinary bladder has been analyzed by de Groat [36], but not at the single-neuron level.

Human repair brain research: A step into high-level human brain research: If one really wants to understand the functioning of the human brain and copy it electronically, one needs to understand the functioning also at the single-neuron level. Figure 15 showed the phase and frequency coordination of neuron firings schematically. By plotting interspike intervals and phase relations of identified neuron firings, one obtains distributions in which changing phase relations can be seen for different natural stimulations (Figure 38). It will be shown that neural subnetworks are organized by phase relations and neural organization changes. The nervous system supplies phase and frequency coordination dynamics rather than particular patterns of 
phase and frequency coordination. Repairs by learning are alterations in the layout of underlying phase and frequency coordination dynamics.

Impaired phase and frequency coordination among $\alpha$ and $\gamma$-motoneurons and secondary muscle spindle afferents, measured with the single-nerve fiber action potential recording method

It was shown that natural impulse pattern can explain pattern change [4] that is to say that the generation of integrative patterns can partly be understood at the single neuron level. Natural impulse patterns were related to integrative CNS organizations. How can this aid in understanding why jumping on springboard contributes substantially to the repair of bladder function? A better understanding can be achieved when going deeper into the complexity of the cooperative and competitive interplay among neurons that means going deeper into the complexity of phase and frequency coordination of CNS selforganization. The phase and frequency coordination of coordinated firing will now be analyzed in more detail.

Time correlations of afferent and efferent impulse patterns are easy to detect in the brain-dead individual as the oscillatory firing of an $\alpha_{2}$-motoneuron $(\mathrm{O} 2)$ was regularly like an inner clock (Figure 12). The phases of fusimotors and spindle afferent action potentials (APs) could be defined with respect to the impulses of that inner clock. In the paraplegic, the rhythmic firing was rather irregular. The motoneuron firing therefore cannot be used any more as a time reference basis. More phases between the extracellular action potentials of the different fibers are necessary to fully describe the correlation between the simultaneous impulse patterns. In Figure 163Cg of [4], the mutual phases between the APs of the different fibers are defined. Since only few phases occurred in a sweep piece of $0.8 \mathrm{~s}$ duration, phases and interspike intervals occurring in certain time intervals were pooled and plotted in A and B of the Figures 38 and 39.

In Figure 38, the interspike intervals (IIs) and the phases are shown for similar time intervals given in Figure 163 of [4]. Before stimulation, within the time interval $1-6 \mathrm{~s}$, the $\alpha_{3}$-motoneuron fired every $100 \mathrm{~ms}$, the $\gamma_{1}$-motoneuron every 100 to $130 \mathrm{~ms}$, and the SP2(1) fiber every 80 to $150 \mathrm{~ms}$ (Figure $38 \mathrm{Aa}$ ). The $\alpha_{2}$-motoneuron mostly fired every $300 \mathrm{~ms}$ and the SP2(2) fiber every $250 \mathrm{~ms}$. At that particular time interval, similar phases (phase relation of broad peak type) occurred twice per $\alpha_{3}$-oscillation period between the APs of the $\alpha_{3}$ and $\gamma_{1}$ axons, between the $\gamma_{1}$ and the SP2(1) fibers, and between the $\alpha_{3}$ and the SP2(1) fibers (Figure $38 \mathrm{Ba}$ ). One phase relation occurred between the impulses of the $\alpha_{3}$ and $\alpha_{2}$-motoneurons, and two between the $\alpha_{3}$ and the SP2(2) fibers. The broad phase relations between discharge patterns are interpreted as interactions between populations of neurons. Following different stimulations interspike interval (II) distributions and phase relations changed with time.

Upon touching sites 1-5 (Figure 163B) [4], the IIs of the almost oscillatory firing $\gamma_{1}$-motoneuron reduced in size to be more similar to those of the oscillatory firing $\alpha_{3}$-motoneuron (Figure $38 \mathrm{Ab}$ ). The changing of the different phase relations indicates changes in the interactions between neuronal sub-networks (Figure $38 \mathrm{Bb}$ ). Upon touching sites 6 to 10 (especially sites 6 , and 7 (inside anal reflex area)), the IIs of the almost oscillatory firing $\gamma_{1}$-motoneuron increased again (Figure $38 \mathrm{Ac}$ ). A transient partial synchronization occurred between the different nerve fibers (Figures $38 \mathrm{Bc} \& 40 \mathrm{Ac}$ ). Upon pin-pricking sites $1-5$, the IIs of the almost oscillatory firing $\gamma_{1}$-motoneuron reduced again to have a similar II distribution as the $\alpha_{3}$-motoneuron. The $\alpha_{3}$ motoneuron even slightly increased its IIs (decrease of activity), so that the II distribution of the oscillatory firing $\alpha_{3}$-motoneuron and the now oscillatory firing $\gamma_{1}$-motoneuron became very similar (Figure $38 \mathrm{Ad}$ ).
Upon pin-pricking sites 6 and 7 (inside the anal reflex area), $a$ and $\gamma$-motoneurons and secondary muscle spindle afferents showed similar II distributions (Figure $38 \mathrm{Ae}$ ). Only one phase relation was organized per oscillation cycle between the different nerve fibers (Figure $38 \mathrm{Be}$, Figure $40 \mathrm{~A}$ e). A synchronization between the APs of the different nerve fibers occurred as can be seen from the direct impulse patterns (Figure163B of [4]). The occurrence of similar II distributions of, and transient constant phases between, the APs of the $a_{3}, \gamma_{1}$ and SP2(1) fibers is interpreted in the way that, in its oscillatory firing the oscillatory firing $\alpha_{3}$-motoneuron built up an external loop to the muscle spindle innervated by the $\gamma_{1}$ and SP2(1) fibers. The $\gamma$-loop became integrated into the oscillatory firing of the $\alpha_{3}$-motoneuronal network. Before pin-pricking, the $\gamma$-loop, consisting partly of the $\gamma_{1}$ and SP2(1) fibers, also contributed to the oscillatory firing, since on the average there existed phase relations. With the pin-pricking, however, the II distributions also assimilated, so that this $\gamma$-loop was directly included into the oscillatory firing of the $\alpha_{3}$-network rather than only contributing to the drive of it. The building up of an external loop to the periphery by spinal oscillators is substantially used when a patient with a spinal cord injury is jumping on springboard (Figure $40 \mathrm{Bg}$ ). Upon pin-pricking sites 8, 9 and 10 (outside of the anal reflex area) and following pin-pricking of site 10, the II distribution of the SP2(1) fiber shifted away from those of the $\alpha_{3}$ and $\gamma_{1}$ axon. The oscillatory firing $\alpha_{3}$-motoneuronal network had abolished its external loop, even though still getting drive from it. Upon anal reflex stimulation and catheter pulling, the external loop was not built up again (Figure 39).

Following touch, pin-prick and anal reflex stimulation, but not painful catheter pulling, mostly two-phase relations existed in paraplegic 9 between the activity of the $\alpha_{3}, \gamma_{1}$ and SP2(1) fibers per oscillation period (100-140ms) of the $\alpha_{3}$-motoneuron (Figures 38,39 ), but the phase relations changed with ongoing time. With the activation of the parasympathetic division, upon bladder catheter pulling, three phase relations occurred per $\alpha_{3}$-motoneuron oscillation period (Figure $39 \mathrm{Bc}, \mathrm{e})$. At the peaks of parasympathetic activation, three phase relations occurred (Figure $39 \mathrm{Bc}, \mathrm{e}$ ), and only two phase relations were present with little parasympathetic activation (times between the peaks 1 and 2) (Figure 39Bd). Even though the functional units, consisting of fusimotor ( $\gamma$-motoneuron) and $\alpha$-motoneuron neuronal networks and spindle afferents fibers, were rather unstable in paraplegic 9 , in comparison to the brain-dead human, an important difference between skin (somatic) and bladder (parasympathetic) stimulation occurred. Another (third) phase relation per $\alpha_{3}$-motoneuron oscillation period occurred with the activation of the parasympathetic division. The activated parasympathetic neuronal network of the sacral micturition and defecation center had channeled input to the oscillatory firing somatic neuronal network.

Phase relation changes between the action potentials of the $\alpha$ and $\gamma$-motoneurons and secondary muscle spindle afferents in paraplegic 9 upon somatic and parasympathetic activation of the sacral micturition center: As shown in Figures 38 and 39 the number and the values of phase relations changed between the firings of the different nerve fibres upon different stimulations. In the brain-dead human HT6, two phase relations were found between the $\alpha_{2}$-motoneuron and the secondary muscle spindle afferent fibre SP2(2) and the $\alpha_{2}$ and the $\gamma_{1}$-motoneuron [73]. Also, in the paraplegic two phase relations often existed between the firings of the different nerve fibres. It may be that a third phase relation occurs when the activated parasympathetic division channelled an additional input to the oscillatory firing somatic neuronal network. It is therefore worthwhile to further analyse the 


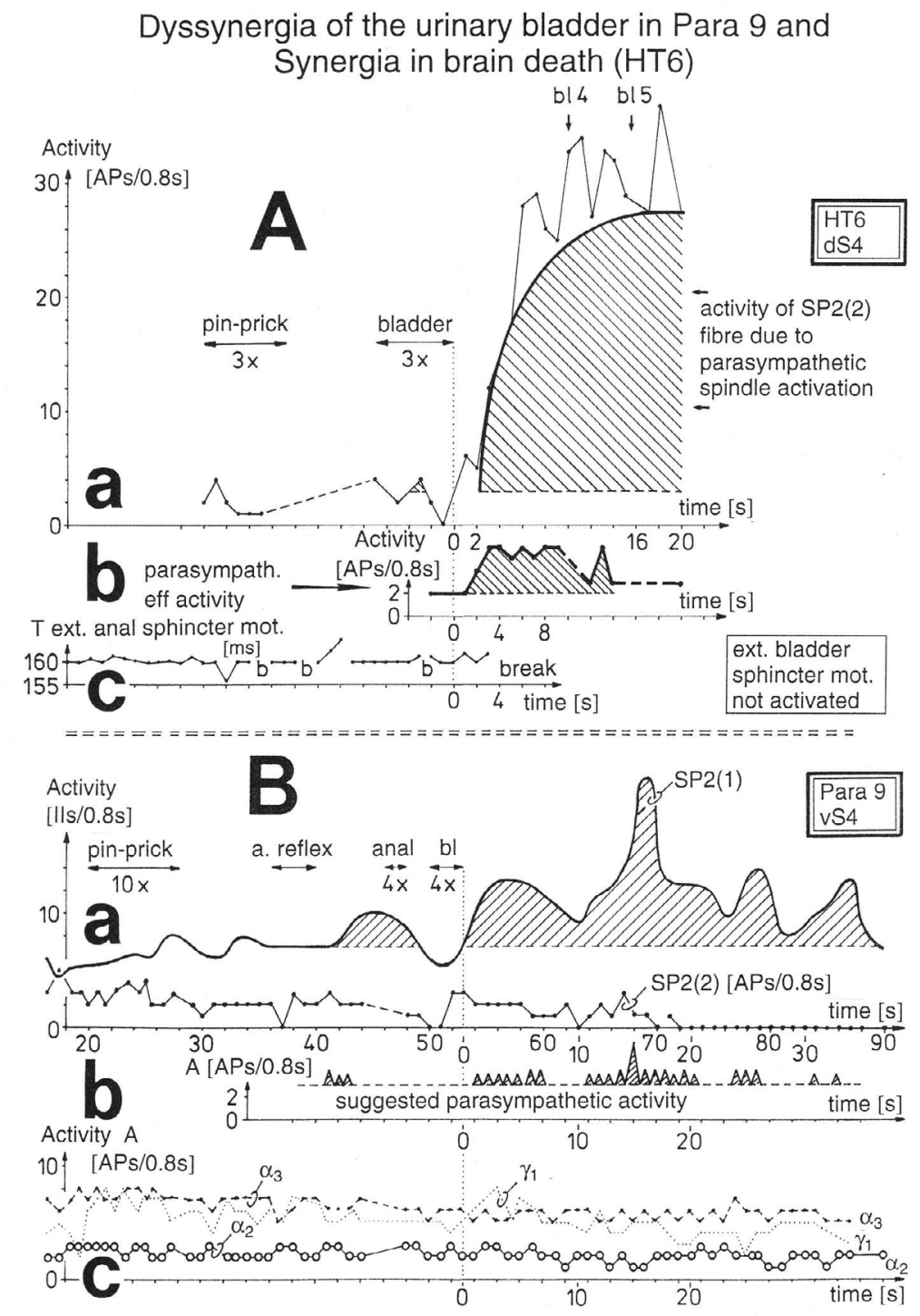

Figure 37. Direct comparison of secondary muscle spindle afferent activity and motoneuron activity between the brain-dead human HT6 with a synergy of the bladder (A) and the paraplegic 9 with a dyssynergia of the bladder (B).

A. Simultaneous measurements of activities of secondary muscle spindle afferents (a), parasympathetic preganglionic motoneurons (b) and oscillatory firing (high activity mode) of a sphincter motoneuron innervating the striated anal sphincter (c). Note that with the transient activity increase of the parasympathetic fibers (b) the secondary muscle spindle afferent fiber increased strongly its activity (a) for minutes, and the oscillatory firing sphincter motoneuron discontinued its oscillation (c) to reduce strongly its activity. bladder $3 \mathrm{x}=3$ times bladder catheter pulling. T ext. anal sphincter mot. $=$ oscillation period of the sphincter $\alpha_{2}$-motoneuron innervating the anal sphincter. For further details, see Chapter $\mathrm{V}$ of [1].

B. Simultaneous measurements of activity of secondary muscle spindle afferent fibers SP2(1) and SP2(2) following anal (anal 4x) and bladder catheter pulling (bl 4x) (a), and the activity changes of an $\alpha_{2}$-motoneuron (FR) and $\alpha_{3}$-motoneuron (S) and a dynamic fusimotor fiber $\left({ }_{1}\right)$ (c). Note that following bladder catheter pulling (and probably parasympathetic activity increase), the spindle afferent fiber SP2(1) (most likely contributing to continence) increased its activity in an undulating manner (a), whereas the SP2(2) fiber did not (probably not connected to continence) (a), and the $\alpha$-motoneurons did not reduce their activity (c). The dynamic fusimotor $\gamma$ transiently increased its activity similarly as in HT6 measurements. In similarity to 'Ab', the suggested parasympathetic activity increase is pictured (b). a. reflex $=$ anal reflex stimulation. IIs $=$ interspike intervals; IIs $/ 0.8 \mathrm{~s}=(\mathrm{APS}-1) / 0.8 \mathrm{~s}$ (the activity measures IIs $/ 0.8 \mathrm{~s}$ and APs $/ 0.8$ s differ by ' 1 ')

number of occurring phase relations per oscillation cycle upon different somatic and parasympathetic stimulations.

Since two phase relations occurred per oscillation cycle between the $\alpha_{3}$ and $\gamma_{1}$-motoneurons and the SP2(1) fiber (Figure $38 \mathrm{Ba}$ ) in paraplegic 9 , and also their IIs were rather similar, it is concluded that the neuronal networks of the $\alpha_{3}$ and $\gamma_{1}$-motoneurons formed together with the spindle afferent fiber SP2(1) a part of a functional unit. The neural ensemble is built by efficiencies of synapses and projections between the convergence of several fusimotors on one muscle spindle and by the divergence of muscle spindle projections onto several rhythmically firing populations of neurons driving $\alpha$ and $\gamma$-motoneurons. Such a functional unit is partly pictured in Figure 40 and schematized drawn by 3 circles in Figure 40A. The $\alpha_{2}$-motoneuron and the SP2(2) fiber belonged to another functional unit (another ensemble) (longer IIs and the existence of only 1 phase relation). The two functional units (ensembles) are characterized in Figure 40A by two sets of three circles each. The two functional units interacted with each other as there existed a phase relation between the $\alpha_{2}$ and $\alpha_{3}$-motoneurons (Figure $40 \mathrm{~A})$. 


\section{Interspike intervals and phase relations of natural firing patterns}
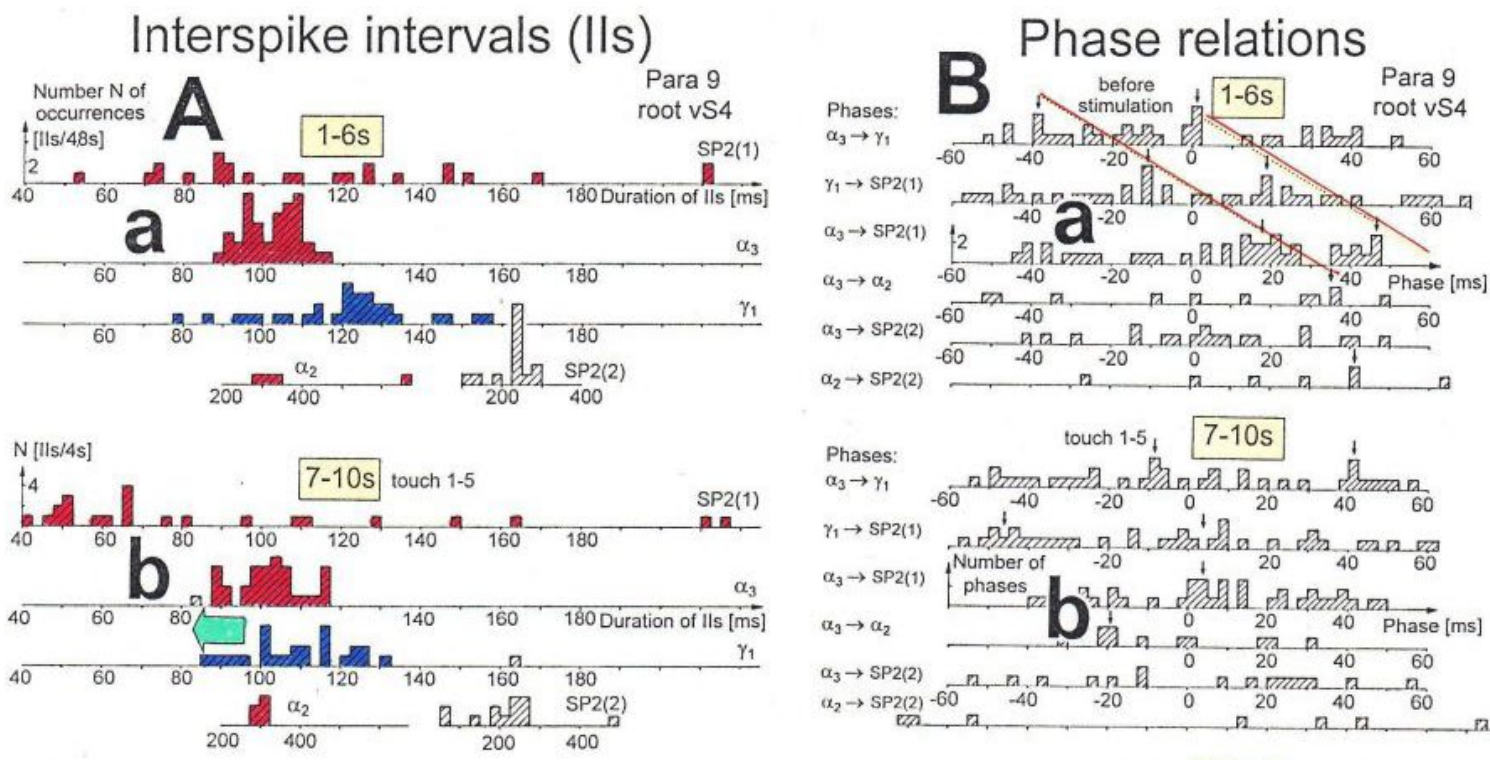

Phases:
$\alpha_{3} \rightarrow \gamma_{1}$
$\gamma_{1} \rightarrow \mathrm{SP}_{2(1)}$
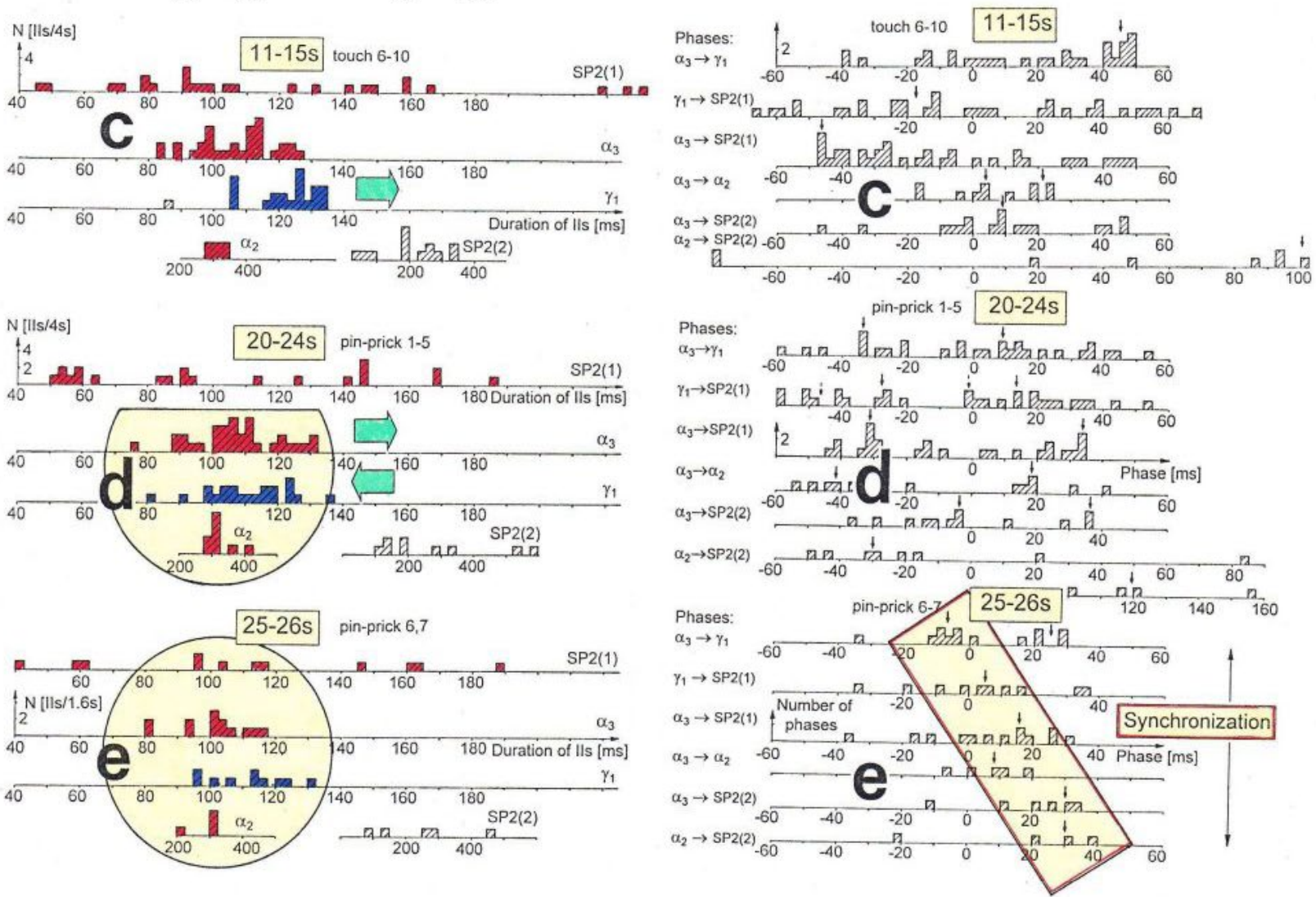

Figure 38. Relative phase and frequency coordination between $\alpha$ and $\gamma$-motoneurons and secondary muscle spindle afferents due to touching and pin-pricking sacral dermatomes as in Figure 9G. A. Interspike interval distribution of spindle afferents SP2(1) and SP2(2), $\alpha_{2}$ (FR) and $\alpha_{3}$-motoneurons (S) and the dynamic fusimotor $\gamma_{1}$ for different time intervals upon touch, pin-prick and anal catheter pulling. Interspike intervals (IIs) were collected from several sweeps of $0.8 \mathrm{~s}$ duration per second. External loop generation and frequency coordination of $\alpha$ and $\gamma$-motoneurons and secondary muscle spindle afferents are marked by the semi-circle and the full circle. The large arrows point to the increase and decrease of the mean II of the distribution. Unsafe identification of $\alpha_{2}$ and $\alpha_{3}$-motoneurons (or vice versa) because of loss of specific oscillator properties. B. Histograms of the phases between afferent and efferent fibers for the time intervals indicated, upon different stimulation. Phases were collected from several sweeps of $0.8 \mathrm{~s}$ duration per second. The small arrows indicate phase relations. Phase coordination is indicated in a,e. Para 9; vS4 

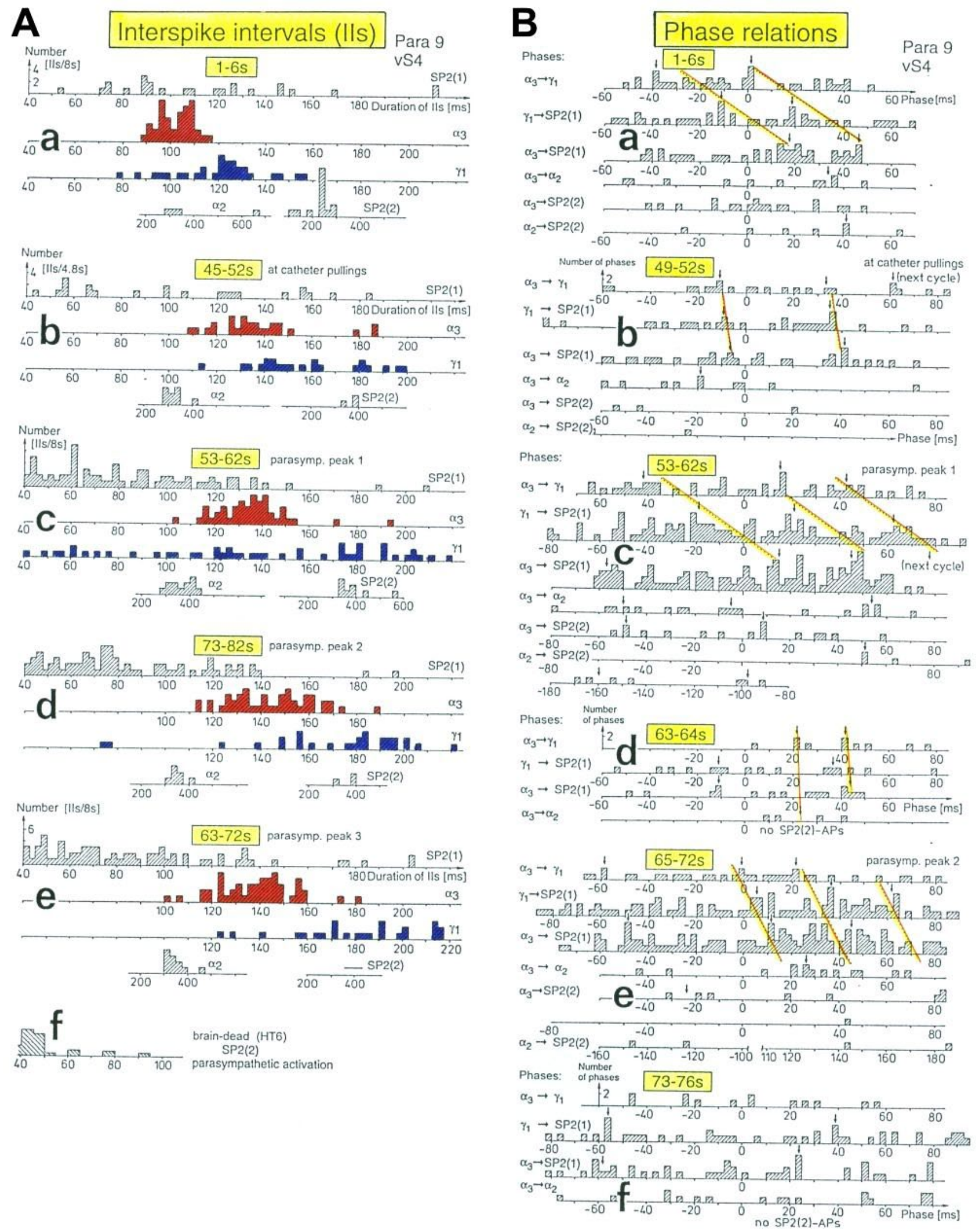

Figure 39. Interspike intervals (IIs) and phase relations for time intervals indicated in Figure 162 of [4]. For legend, see Figure 38 
Before stimulation (but with the anal and bladder catheters positioned), there were two phase relations in unit 1 When touching sites 1 to 5, only slight changes occurred in the two units with respect to the number of phase relations (b). But when touching sites 6 to 10 , a partial synchronization occurred (c) and functional unit 1 reduced the number of phase relations to one. When pin-pricking sites 1 to 5 , two phase relations occurred again in unit 1 (d). Upon pin-pricking sites 6 and 7, the number of phase relations between all the components of the two units dropped to one (e), and synchronization occurred between the firing patterns (Figure 163B of [4]). Since in the braindead human two-phase relations per oscillation cycle were observed in the functional units [73], it is possible that synchronization and the existence of only one phase relation for 2 to 3 seconds reflected a slight pathologic organization of the networks.

Even though upon touching sites 6 to 10 (Figure 40A c) or upon pin-pricking sites 6 to 7 (e) only one phase relation existed in unit 1, and synchronization occurred with both stimulations, it was shown [4] that the touch afferent input organized a different functional state of unit 1 than pin-pricking. The response time till the shortening of the oscillation period was longer than the oscillation period $(\approx 100 \mathrm{~ms})$ for pin-prick and shorter for touch. It was shown [4] that repetitive touch stimulation (most effective inside the anal reflex area) reinforced the sustained stretch reflex of the anal sphincter (continence pattern), and repetitive pin-prick stimulation replaced the continence pattern by the protection reaction of the anal sphincter. The number of phase relations alone therefore only provides limited information on the functional state of the organization of the neuronal networks of the human spinal cord. Measurements of a number of parameters are necessary to yield a rather complete description of the functional state of neuronal networks.

Following pin-prick 8 and 10 and with no stimulation two phase relations existed again in functional unit 1 (Figure $40 \mathrm{f}, \mathrm{g}$ ), in some similarity to pre-stimulation status (a). Following two anal reflex stimulations, partial synchronization occurred in the components of the two units, and mainly two-phase relations existed (h). But the organizational state was still not very similar to the pre- (a) or poststimulation state in unit $1(\mathrm{~g})$, since the parasympathetic division was slightly activated following anal reflex stimulation as was measured by the impulse pattern (increase of doublet activity) of the secondary muscle spindle afferent fiber SP2(1). Therefore, probably one phase relation was due to the somatic activation in similarity to Figure 40 Ac, e and the other phase relation was due to the activation by the parasympathetic division. During bladder catheter pulling (i) and with no stimulation $(\mathrm{k})$, the number of phase relations and possibly the functional organization, was again similar to the pre-stimulation state (a).

Following strong (painful) bladder catheter pulling with a strong activation of the parasympathetic division (time interval 53-62s (j)), measured by the increased doublet firing (for doublet firing see page 514 of [3]) of the SP2(1) fibre, the functional organization of the sacral micturition centre of the disconnected spinal cord changed completely. Functional unit 1 was now correlated by three phase relations per $\alpha_{3}$ oscillation cycle. The functional unit 2 also showed 3 phase relations per an $\alpha_{2}$-oscillation cycle and interacted with functional unit 1 by 3 phase relations as well (between the $\alpha_{3}$ and $\alpha_{2}$-motoneurons; Figure 40Aj (5362s)). For further details see the book 'human neurophysiology' [3].

Even though, due to different natural stimulations (touch, pain, catheter pulling), the phase relation changes are very complicated in neural sub-networks and the communication among them and the doublet firing of secondary muscle spindles has to be taken into consideration [3], it is really possible to analyze/understand neural network organization at the neuron level. Computer programs could help. The human neural networks organize themselves by the numbers and values of phase and frequency coordination's. Their impairment has consequences in CNS functioning and has to be repaired by movementbased learning.

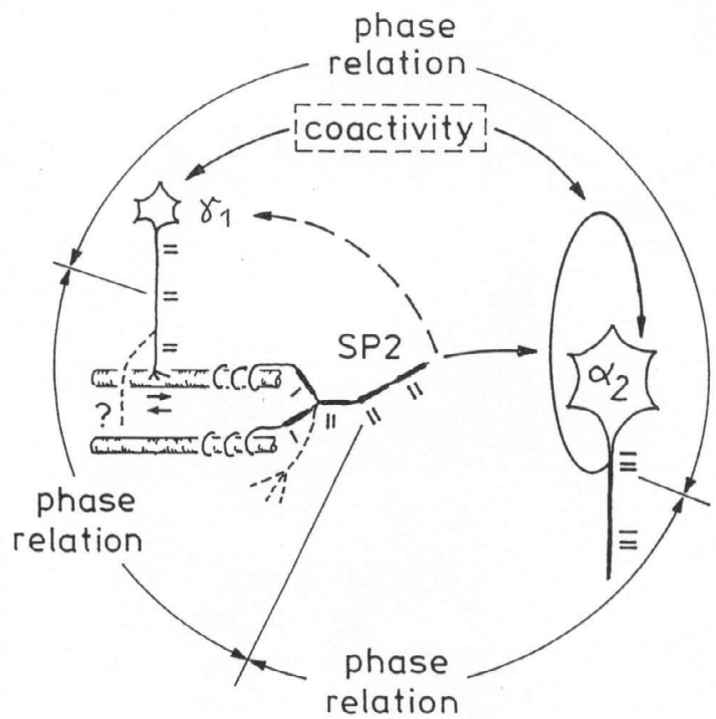

Figure 40. Schematized existing phase relation between $\alpha_{2}$ and $\gamma_{1}$-motoneurons and a secondary muscle spindle afferent fiber (SP2). Parallel existing phase relations between other parent afferents and the $\alpha_{2}$-motoneuron and between parent secondary spindle afferents are not shown. Phase relation means, the increased occurrence of phases in $\mathrm{ms}$ in a certain phase range between the action potentials (APs) of the two compared nerve fibers. The complex afferent and efferent muscle spindle innervation was not tried to show. Small arrows at intrafusal muscle fiber indicate local contraction, which is in nuclear chain fibers readily transmitted to the place of afferent innervation. A possible reason of the doublet firing of the SP2 fiber is pictured to occur from single APs (schematized by bars) of two myelinated endings, not necessarily from pacemaker switching. More endings of the parent SP2 fiber and $\gamma_{1}$-motoneurons are indicated by dashed line branches. "Coactivity" indicates a correlation between $\gamma$ and $\alpha$-motoneuron spinal cord circuitries for higher activations

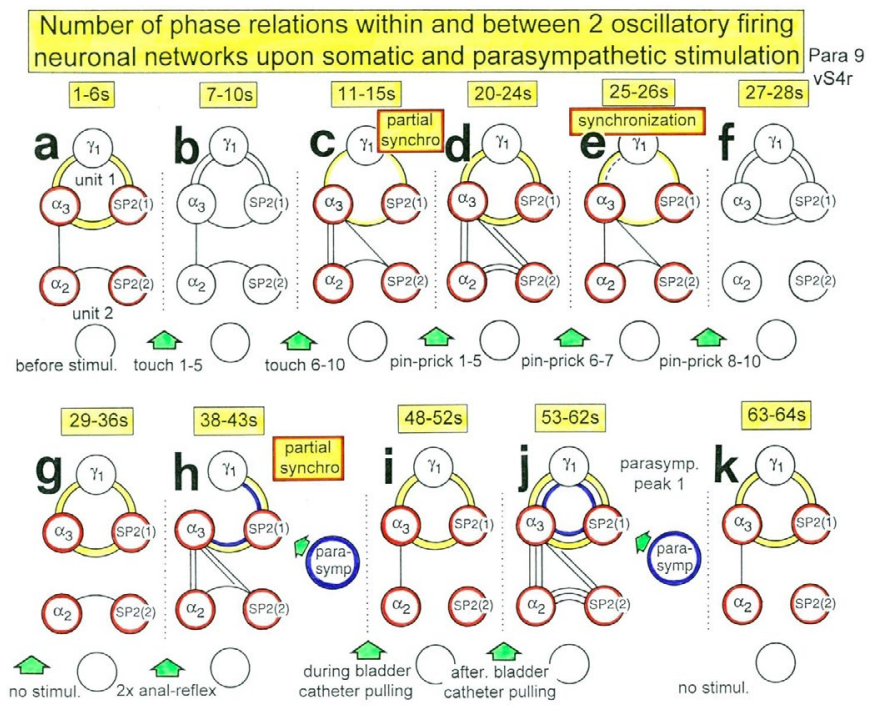

Figure 40A. Number of phase relations within and between the two functional units $\alpha_{3}$ $\gamma_{1} / \mathrm{SP} 2(1)$ and $\alpha_{2} /$-/SP2(2). Note that in 'a' the functional unit 1 is with two phase relations per oscillation period in a stage similar to those seen in the brain-dead individual; with synchronization only 1 phase relation occurred (e) and the parasympathetic division channeled an extra phase relation to interact with the somatic division (j) 
Need to improve the stability of phase and frequency coordination to allow specific pattern formation and learning transfer: A young mother, with stress incontinence after giving birth to her first child, could strongly improve her continence status by jumping on springboard in addition to other training. Her CNS was not injured. It was the periphery that required repair by means of changing the CNS.

In severe cervical spinal cord injury, however, solely jumping on springboard is not sufficient for bladder repair. First, of course, the patient has to regain movement functions back (especially the trunk stability) to be able to perform the jumping on springboard. Further, the self-organization of CNS networks by phase and frequency coordination has to be improved to make learning transfer from movements to bladder functions possible, since in every CNS injury the phase and frequency coordination is impaired. Large instabilities in phase and frequency coordination will not allow specific pattern formation as a basis for learning transfer. However, the stability of phase and frequency coordination can be improved when the patient is exercising on special coordination dynamics therapy device.

Building up of external loops to the periphery by premotor spinal oscillators: It will be shown now that with the building up of simultaneous phase relations between $\alpha, \gamma$ and SP2 fibers and the assimilation of II distributions (coordination's of rhythms), an external loop of premotor spinal oscillators is built up to the periphery, which makes it possible to directly influence the firing of spinal oscillators by a rhythm training. The somatic and parasympathetic pattern organizations in the sacral micturition center can simultaneously be entrained by jumping on springboard (Figure 40B g) (including the stimulation of movement (f) and bladder receptors) to allow movement-based learning in the continence and movement patterns and to induce learning transfer from movements to urinary bladder functions. A repair of neuronal network patterns of the functionally disconnected sacral micturition center in spinal cord injury is possible.

External loop of premotor spinal oscillators and rhythmic, dynamic stimulation of motor and bladder functions: While jumping on a springboard (Figure 40B g) (and other rhythmic movements like sky-walking or running) premotor spinal oscillators organize themselves to fire transiently in an oscillatory manner according to the motor pattern and build up an external loop to the periphery (Figure 40B). If the frequency of the rhythmic movement has an integer relationship to the Eigen-frequencies of the premotor networks and more rostral networks, these premotor networks get entrained for more specific self-organization. When jumping on a springboard (g) not only the motor networks get activated; also, the external sphincteric motoneurons, innervating the external bladder and anal sphincter, as a part of the pelvic floor, are rhythmically activated to counteract the rhythmic weight changes of the intestine. Further, the rhythmic, dynamic, stereotyped up and down movements stimulate stretch, tension, flow, and mechanical receptors of the bladder (detrusor and proximal urethra). This rhythmic movement-related sensory input with $\approx 1 \mathrm{~Hz}$ bears similarity to the sensory input stimulated by bladder and anal catheter pulling with $\approx 1 \mathrm{~Hz}$ during the measurements (Figure 39). Repetitive phase relation changes in and among neural ensembles will occur in some similarity to the changes following catheter pulling (Figure 39B). Since the neurons involved in the generation of movement and continence (and micturition) patterns (especially if the neurons serve both functions at the same time) are synchronously, rhythmically activated, the pathologic bladder patterns get entrained from the rather physiologic jumping movement, in some similarity to co-movement. The synchronized activation of the somatic and parasympathetic networks allows efficient learning transfer, since the neurons work as coincidence or more general as coordination detector (Figure 49). If there is fluid in the bladder and material in the bowel and rectum, the continence stimulus is stronger. Also walking and running will stimulate and change the intrinsic dynamics of the micturition and defecation centers, but not as strong as the jumping on springboard. The walking and running on a treadmill in severe cervical spinal cord injury is performed under weight support, whereas during jumping on springboard no weight support is needed (Figure $40 \mathrm{Bg}$ ). The sympathetic nervous system division, probably innervating the internal urinary bladder sphincter (smooth muscle), will also be activated synchronously. Since the frequency of jumping is around $1 \mathrm{~Hz}$ similar to the 'Eigenfrequency' of the $\alpha_{3}$-motoneuron oscillators, these oscillator networks should be entrained most efficiently.

Urinary bladder repair through CDT: The repair of the urinary bladder functioning by reorganization of networks seems to be most difficult because there is false neuronal network organization in the parasympathetic nervous system (overactive (spastic) detrusor) and the somatic division (spastic external (striated) bladder sphincter), and there is false interaction of the interlacing somatic and parasympathetic networks (detrusor-sphincteric dyssynergia: when the detrusor contracts, the external sphincter is also contracting instead of opening (relaxing)). But because the neuronal networks of the somatic and the parasympathetic nervous systems are interlaced and interact with each other, it should be possible to improve parasympathetic functions when improving somatic functions by CDT. Since humans have a high capability for learning, it is possible to repair the injured networks by learning. We only have to 'tell' the CNS by training what it has to repair by learning.

The movement-based learning therapy (CDT) partly recapitulates the development. But the learning process is hampered by the deficiency of the CNS injury. To teach the injured CNS to repair itself by trial and error elimination processes, the CNS has, in similarity to the development, to recognize upon CDT, which subnetworks, regulation units, subloops or tracts are not functioning properly (or are missing) and to repair them by error elimination, including the possibility that other brain parts take functions partly over and subnetworks and tract fibers built anew to a limited extent.

According to the ontogenetic landscape for locomotion (Figure 41) [37] newborn stepping (stepping automatism) and treadmill stepping lead to walking with support and finally to walking and running.

A similar attractor layout was obtained for the repair of urinary bladder functions in a 16-year-old female patient (Kadri) with a cervical spinal cord injury (injury approximately 95\%) (Figure 42) [38]. The bladder repair needed over two years of continuous intensive CDT. Changes of functions of the detrusor (bladder) and the external and internal bladder sphincters are extracted from a detailed anamnesis and are pictured by an evolution of the attractor layout with the re-learning of bladder functions.

Repair of sexual functions and bowel peristalsis: In spinal cord injury, the biggest problem is the repair of the urinary bladder, followed by the repair of the sexual function and locomotion. When exercising on the special CDT device (Figure 1) and jumping on springboard (Figure 34), urinary bladder functions are repaired. But since the sexual functions are also located in the lower spinal cord (Figure 35), they are simultaneously repaired by learning transfer from movements in accordance with the experience that fitness training improves potency in men. In similarity to the bladder function repair, where the sacral 

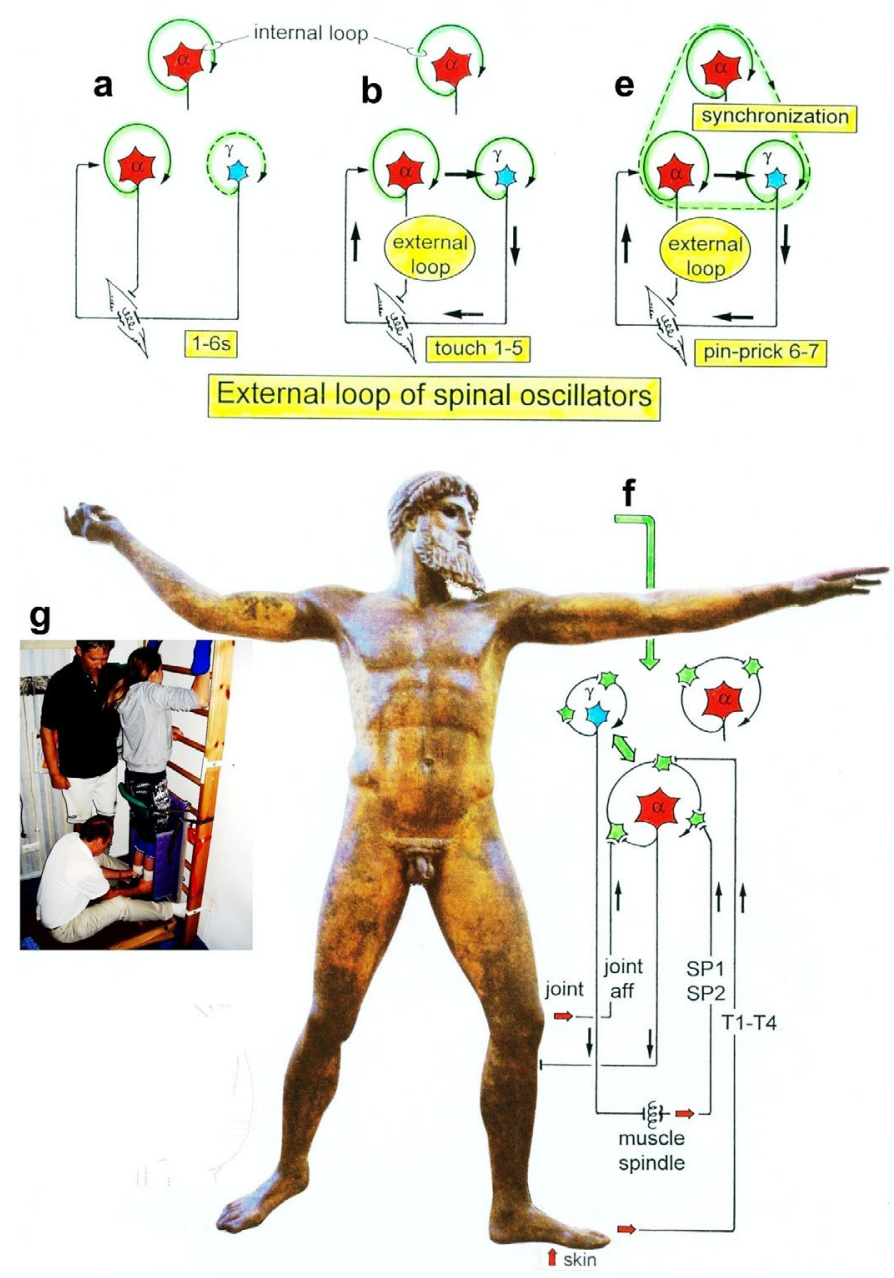

Figure 40B. Spreading of oscillatory firing from $\alpha$-motoneuron neuronal network to include muscle spindles (periphery) and synchronization of different $\alpha$ and $\gamma$-motoneuron neuronal networks caused by touch and pin-prick stimulation.

(a) $\alpha$-motoneuron neuronal networks fired oscillatory (solid line loop), $\gamma$-motoneuron neuronal network did not or did only partly (dashed line loop), upon no additional stimulation; taken from Figure 38.

(b) Oscillatory firing $\alpha$ and $\gamma$-motoneuron neuronal networks built up a phase relation with muscle spindle afferents and efferents (external loop to the periphery, indicated by thick arrows) upon touch

(e) Oscillatory firing $\alpha$ (internal circuitry loop) and $\gamma$-motoneuron neuronal networks (external loop) synchronized (broad peak phase relation) upon pin-pricks 6-7. The dashed line loop represents synchronization.

(f) Oscillatory firing $\alpha$ (internal circuitry loop) and $\gamma$-motoneuron neuronal networks (external loop) are extended by analogy from the continence muscles to muscles for locomotion. The open arrows indicate that it may be possible to synchronize spinal oscillators by rhythmic afferent input, generated by rhythmic movements (such as jumping on a springboard or running), and to re-preformat the neuronal circuitry by synapse remodeling to fire more physiologically oscillatory to reduce spasticity and improve locomotion. Extensive pathologic movement like tremor may entrain neuronal circuitry to increase tremor movement.

(g) Supported jumping on the springboard in anti-phase. The patient with the severe cervical spinal cord injury is supported by the author.

The Greek good is a bronze statue of Zeus found close to the cape of Artemision 460 BC

micturition center also has to be connected to the pontine micturition center, also the sexual functions of the lower spinal have to be connected to supraspinal centers by integrative movements.

Since there is learning transfer from somatic (movements) to vegetative functions, impaired bowel functions are repaired, when exercising on the special CDT device. The peristalsis is repaired. It needs approximately $30 \mathrm{~min}$ exercising on the special CDT device to improve the peristalsis in the short-term memory. Repeated exercising will repair the peristalsis in the long-term memory in similarity to the reduction of spasticity (Figure 27).

Lowering of increased blood pressure - improvement of cardiovascular performance: As directly measured in a patient with an extremely injured brain (the coma patient Manolis of below), the cardio-vascular performance was repaired in the short and long-term memory [39]. But the cardio-vascular performance can be improved also in rather healthy humans. Figure 43 shows the lowering of the resting blood pressure of the Author when exercising on a special CDT device (Figure 43).

As can be seen from Figure 43, a rather healthy fit person over 70 probably has a transient resting blood pressure lowering for approximately 8 hours. This healthy lowering of the blood pressure could be used for patients with hypertension. When exercising every 6 hours for $60 \mathrm{~min}$ (2000 to 3000 turns), including transiently against higher loads, he can probably lower his resting systolic blood pressure by 10 to $20 \mathrm{mmHg}$. If he has no adverse heart problems, he does not need to exercise at night, but if he has, maybe he should also train once at night. Most elderly anyway get up at night. It would be no problem for them to exercise also at night. An exercise session before going to sleep most likely increases the length and deepness of the sleep. When performing antihypertension exercise treatment regularly, it may be sufficient to exercise only for $30 \mathrm{~min}$ at a load of $30 \mathrm{~N}$, to lower the systolic resting blood pressure by 10 to $20 \mathrm{mmHg}$.

Inhibition of cancer growth: One of the most important benefits of CDT is the inhibition of cancer growths. Epidemiological data document that regular exercise protects against the development of certain cancers (especially breast cancer) and lowers the risk of disease recurrence $[40,41]$ in contradiction to the previous opinion not to exercise so that the immune system has all the power to fight against the cancer. Higher levels of physical activity have a reduced risk of developing a variety of cancers compared to those who engage in lower levels of physical activity [40].

The anti-cancerous effects of physical activity are believed to depend on the intensity, type, frequency, and duration of the activities $[42,43]$. Proposed mechanisms include DNA repair, immune function, endogenous hormones, and low-grade inflammation [44-47].

Exercise may directly suppress tumor growth, as suggested by the decreased risk of disease recurrence in physically active cancer patients. A likely mechanism behind this cancer protection across a range of cancer diagnoses seems to be the mobilization of cytotoxic immune cells during exercise.

The contribution of CDT to cancer protection by physical activity [48] is the improvement of the cardio-vascular performance, including the lymphatic vessel system, and may be the DNA repair. Exercise may work both on the mobilization of NK (natural killer) cells, and on the tumor microenvironment to generate a NK cell activating milieu [47]. The inhibition of cancer recurrence by CDT includes also the improved microcirculation of blood and lymphatic vessels, because the immune cells have a better access to the cancer cells and metastases in the lymphatic system. The main action when exercising on the special CDT device is the improvement of the peristalsis of the lymphatic vessels to improve the microcirculation in similarity to the improvement of the peristalsis in the bowel and the improvement of the function of the somatic muscles in the blood vessel walls, especially of the arterioles.

The Author himself suffered a 'squamous cell carcinoma (epithelioma)' (a malign tumor) in the maxilla (stage between 1 and 2) and became interested in cancer protection treatment. The tumor 


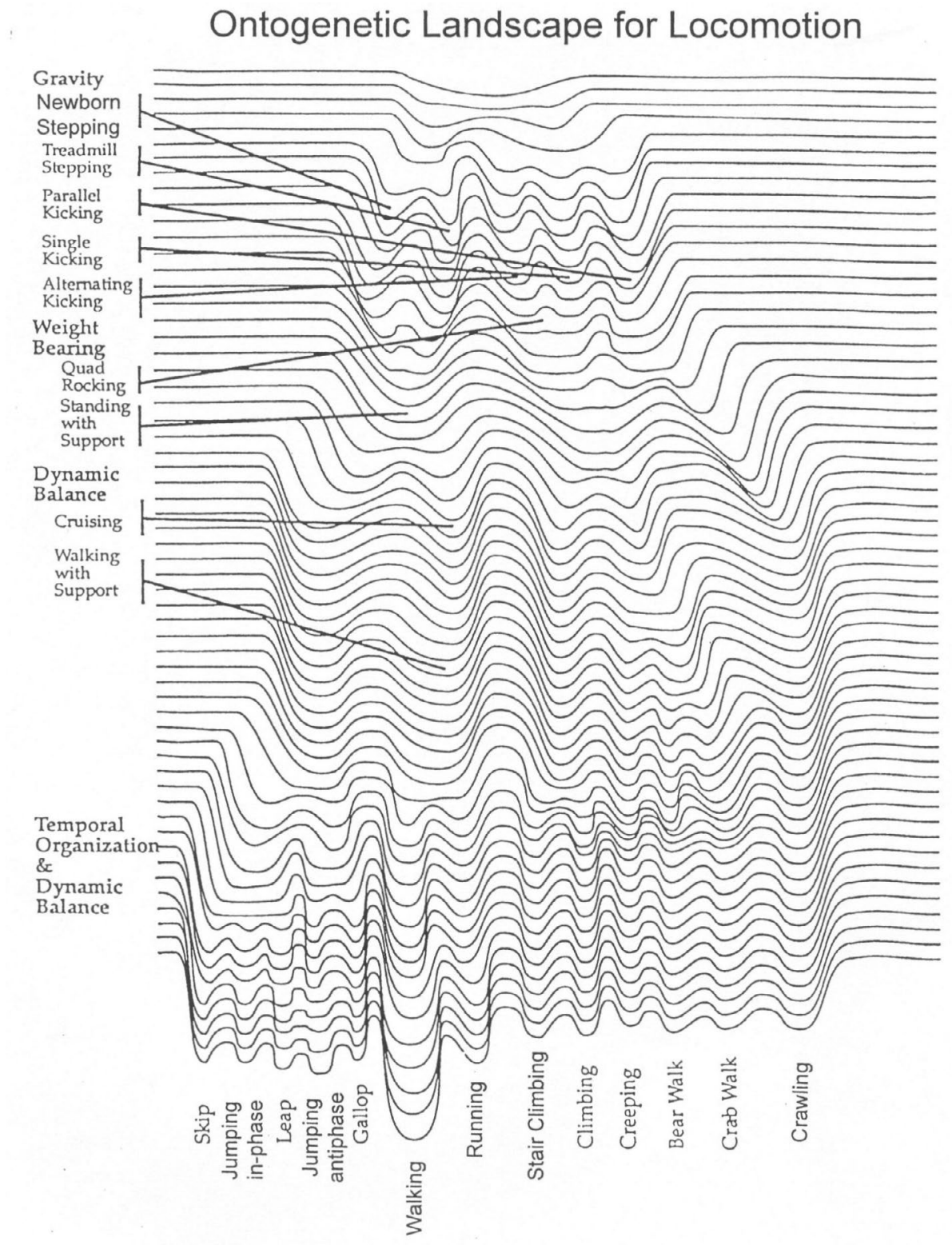

Figure 41. Ontogenetic landscape for locomotion. The evolution of the attractor layout (see System Theory of Pattern Formation) for the different movements. With permission of Esther Thelen $(\dagger)$

was removed by surgery and a neck dissection performed: Two lymph nodes with formation of metastases were removed and two further stages of lymph nodes and lymph vessels were removed for safety reasons. Radiation therapy and chemotherapy were administered to the tumor area to reduce the risk of tumor recurrence from $30 \%$ to $15 \%$. To get rid of the side effects of chemo and radiation therapy, the Author started CDT with approximately 15 hours per week. He is now a 10-year-cancer survivor, but still did not get rid of all the side effects. If the Author would have known at the time of cancer treatment that physical activity protects against cancer recurrence, he may not have accepted radiation and chemo therapy. For further see below under 'Authors own experience with the anticancer effect and body function repair through CDT'.

The exercising on the special CDT device reduces also edema in the axilla of patients after breast cancer treatment including the removal of the lymph nodes of the axilla. For further details see [48].
Different views of CNS repair: The repair physiology has several aspects of learning:

1. The repair can be seen as movement-based learning. We relearn the impaired movements and by re-learning the movements the CNS is repaired. By learning transfer we repair also those functions, which cannot be trained directly, as for example urinary bladder functions. By plasticity, other not-injured parts can take function over from severely injured or lost brain parts. By training of movement patters which animals used during phylogenesis (Figure 44), may be damaged developmental older parts can be reached for repair, which seem to be difficult to repair.

2. But we can also see the repair differently, namely by trial and error elimination processes. We train different movements or other patterns and the nervous system recognizes where there are errors in the organization and by repeated pattern performance, the errors are 
Development of attractor 'Urinary bladder function' upon Coordination dynamics therapy

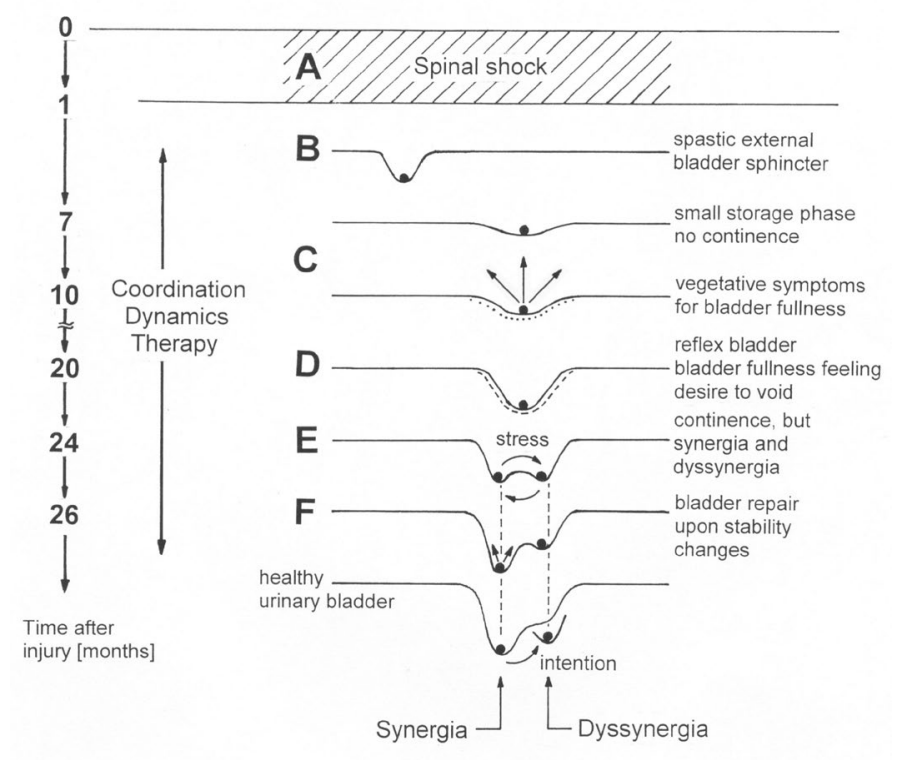

Figure 42. Evolution of the attractor layout of bladder functioning induced by learning transfer from movements to bladder functions upon CDT. The region around each local minimum of the potential landscape acts like a well that weekly traps the system into a coordinated state. Black balls correspond to stable minima of the potential. With learning, the pattern 'spasticity of the external bladder sphincter' vanishes and the patterns for bladder functioning ('synergia' and dyssynergia') appear anew and gain their physiologic stability (physiologic deepness of each basin of attraction). The corresponding attractor layout for physiologic bladder functioning is given. Fluctuation of pattern state (the black ball) (C), and their decrease (F), due to the impairment of phase and frequency coordination of neuron firing, is pictured in ' $\mathrm{C}$ ' and ' $\mathrm{F}$ ' by long and short arrows. Dotted and dashed lines indicate the re-occurrence of bladder sensation. Note that more than two years of optimal continuous CDT were needed for bladder repair

eliminated. We 'tell or teach' the nervous what is pathologic, and the CNS has to repair it by itself. But what are these errors which have been done during development or later on by damage or degeneration? Missing of neural networks, instable networks or too simply made networks with too little variability? This repair strategy is in similarity to the development. Small mistakes done by the execution of the genetics are corrected 'en route' by different movements, as walking, running, jumping and training balance. If nowadays children are only switching between fast food, sweeties and smart phones, they get overweight, move too little and the correction 'en route' during development is not sufficiently taking place and when getting older, they get diseases because of the not correction of the developmental mistakes. For clarity, a movement pattern needs variability to adapt to the surrounding, but the phase and frequency coordination should have little variability.

3a. For functional reorganization, as changing of synaptic efficiencies, and growing of axonal and dendrite trees, we need already genetics to produce proteins. But with neurogenesis more repair is possible.

In animals, adult neural stem/progenitor cells (NPCs) can intrinsically sense excitatory neural activity and thereby implement a direct coupling between excitation and neurogenesis [74-77]. This finding in animals is supported by human research, namely that to a limited extent new motoneurons could be built in the human spinal cord to activate finger functions [78]. The activation of excitationneurogenesis coupling is sensed in animals via $\mathrm{Ca}^{2+}$ channels and
NMDA receptors of NPCs [77]. The activity-dependent neurogenesis supports the re-learning of lost pattern functions and supports clearance of post-injury developed pathologic patterns. The Excitationneurogenesis coupling is influenced by local activity, access to local activity and ability of the local environment.

It is of interest to determine the factors in more detail that create the conditions for excitation-neurogenesis coupling to occur. Cells with neurogenic potential exist elsewhere in the brain, including the neocortex, but contribute to neurogenesis only in the sub-ventricular zone and the dentate gyrus sub-granular zone. However, when cells from non-neurogenic areas are removed and transplanted into neurogenic areas, neuronal progeny results [77]. There are a number of factors that may contribute to the neurogenic potential to induce competence for excitation-neurogenesis coupling in the resident population of NPCs:

a. Type of (natural) local neuronal activity.

b. Access of the NPCs to the local activity.

c. Ability of the local environment to induce activity-sensing competence in the NPCs.

d. Factors intrinsic to the resident NPCs themselves.

Through CDT, a few new nerve cells (motoneurons) could be built from endogenous stem/progenitor cells in a patient with a cervical spinal cord injury. This resulted in the recovery of some finger functions [78]. Exogenous stem cell therapy, by contrast, is unlikely to be effective. This is because the administered cells have to proliferate and have to be integrated into the injured adult neural networks. But this proliferation and integration does not seem to be possible in humans, because physiological neural activity is required to stimulate the membranes of the administered stem/progenitor cells for proliferation and integration to occur (Chapter VI of [3]). Secondly, the communication distance between nerve cells during development and repair seems to be in the range of $0.001 \mathrm{~mm}$, which is difficult to achieve during exogenous stem

\section{Time course of lowering of blood pressure}

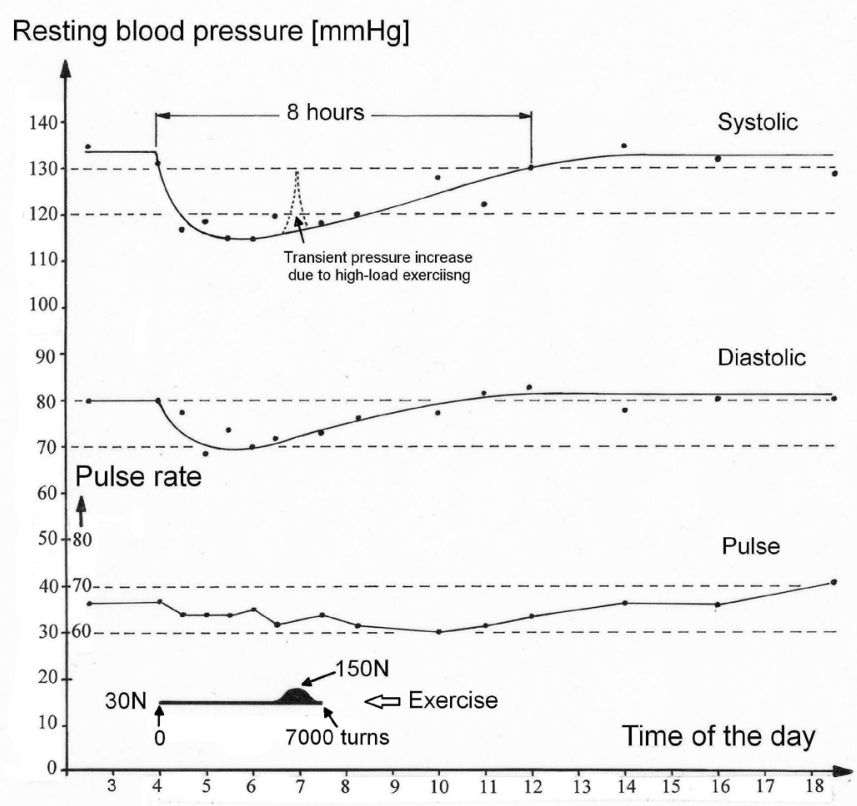

Figure 43. Time course of the lowering of the arterial resting blood pressure when exercising at $30 \mathrm{~N}$ (and transiently up to $150 \mathrm{~N}$ ). Note, measured transient pressure increase due to high-load exercising (dashed line) is indicated 


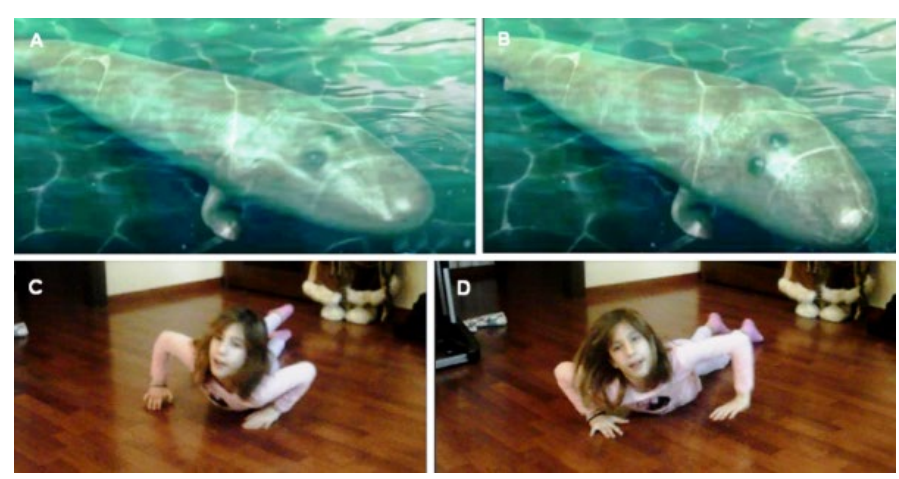

Figure 44. A, B. Possible rotational body movement of Tiktaalik, caused by alternately using one front limb for forward locomotion. C, D. This front limb movement is simulated by a patient with a spinal cord injury (Nefeli) by using alternately the right and left arm. Tiktaalik roseae is a lobe-finned fish from the late Devonian period, about 375 million years ago, having may be features akin to those of four-legged animals (tetrapods). Tiktaalik has a possibility of being a representative of the evolutionary transition from fish to amphibians

cell therapy (Chapter 1 of [3]). Administered stem/progenitor cells which are not adequately stimulated for proliferation and integration will die.

$3 b$. To generate repair in the nervous system, it is likely that permanent changes in gene expression patterns are achieved through permanent changes in chromatin remodeling without changes in DNA sequence. The concept of chromatin remodeling addresses a key challenge of how stable changes in gene expression are induced [79] in neural networks to produce long-lasting changes in repair. DNA methylation is one of the many epigenetic modifications that can alter gene expression. Dynamic and reversible DNA methylation may be essential for learning and memory formation and could transmit repair influences onto adult neurogenesis.

Understanding the complex epigenetic regulation of neural activity and adult neurogenesis is integral to designing therapeutic approaches to restore neurogenesis and cognitive functions. It will also give a tremendous insight into understanding how certain environmental or pathological influences, such as stress, physical activity, depression and epilepsy regulate adult neurogenesis [80].

Excitation-neurogenesis coupling in adult neural stem/progenitor cells [77] can be achieved through depolarization of membranes generated by physiologic movements. During physical activity therefore also, those sites have to be activated which are injured or malformed. In the case of the spinal cord injury patient Nefeli with an injury at the segments Th10/11 (Figure 46), also these spinal cord segments have to be activated for example by trunk rotation movements.

The complexity of the epigenetic regulation is tremendous. Figure 45 shows steps of epigenetic regulation induced by specific and coordinated physical activity, namely movement-based learning (Figure 45). A very important further repair possibility is to correct genetical mistakes by movement-based learning. Can the gene expression get changed in a way that gene defects are not activated? The epigenome can be changed. But is it also possible to change gene expression in a way to inhibit mistakes in the genetic code? Or is it only possible to repair afterwards the mistakes caused by the gene defects?

\section{Clinic: CNS repair achieved and consequences for general health}

General: After performing approximately 15 years of mainly human neurophysiology, the practical part of CNS repair was achieved step by step. The first patient was trained for 6 weeks. Later on, treatment up to many years was administered. No special
CDT device was used at the beginning. By learning from the patient's deficits and improvements, the treatment was improved, and the theory refined. The progress in repair depended on the severance of the injury, the efficiency of the treatment, the aggressivity of treatment and the duration of the treatment. Since CDT is a (movement-based) learning treatment, years of learning are needed as in high-school education. With the successful cancer growth inhibition treatment, the brain repair of a patient being in the permanent coma for several years and the reduction of high blood pressure, a further step of general health improvement was achieved. It seems thus possible to live longer with a better quality of life of up to 20 years.

After offering the achieved CNS repair so far for different groups of patients, the current treatment level is displayed for different diseases of single successfully treated patients. To most of these patients, CDT is still administered to help the patient and their parents and learn about long-term outcomes, which are important especially for children. Long-term outcomes of up to 20 years are not performed in animal research and are also not funded.

CNS repair and health improvement achieved so far: It has been shown that CDT can improve or repair central nervous system (CNS) functioning after stroke [49], traumatic brain injury [50,51], spinal cord injury [52,53], cerebellar injury [54], cerebral palsy [55], hypoxic brain injury [56], in Parkinson's disease [57], spina bifida (myelomeningocele) [58] and scoliosis [59]. Speech had been induced and improved in a patient with severe cerebral palsy [4] and urinary bladder functions were repaired in patients with spinal cord injury $[3,38]$. In patients with cancer, especially breast cancer, cancer growth inhibition could be achieved via CDT [48]. The cardiovascular performance was repaired in a patient with a very severe brain injury (Manolis) during the first years of CDT [39]. It seems therefore that CDT can improve CNS functioning in every case, even in the extreme case, and since the nervous system is involved in nearly all body functions, CDT can improve/repair human health in general.

Spinal cord injury repair in a child: The 5.5-year-old Nefeli suffered a spinal cord injury at the level of Th10/11 (Figure 46,47,48AB) following a cancer operation by medical malpractice. At an age of 9 years CDT was started to inhibit cancer recurrence and to repair the spinal cord injury (SCI). The motor functions improved through CDT. Urinary bladder functions could mainly be repaired, and she re-learned to walk again (Figure 48C, D) with deficits in performance and stability $[60,61]$. The peristalsis of the bowel was repaired. There was even some regeneration of the spinal cord at an age of between 9 and 10. There was no cancer recurrence (neuroblastoma) so far in the following 7 years. The cancer treatment through CDT was therefore successful, the spinal cord repair only partly.

Following approximately 2 years of CDT no real further progress was achieved any more in the repair of the spinal cord. But why did the spinal cord regeneration arrest. There are several possible reasons. Repair also includes mechanisms through long-lasting chain of events of trial and error elimination processes (1). Since Nefeli was only trained in intervals of 3 months, the repair through long-lasting chain of events did not take place. Further, it is possible that there is much more repair below the age of 10 years than later (2). Also, during puberty children with CNS injury loose regained functions (3). But treatment has to be continued to stop the patient's CNS to get worse. Apart from the limited regeneration of the spinal cord when being younger than 10, the repair was mainly due to a re-wiring of the remained tract fibers (4). Most 


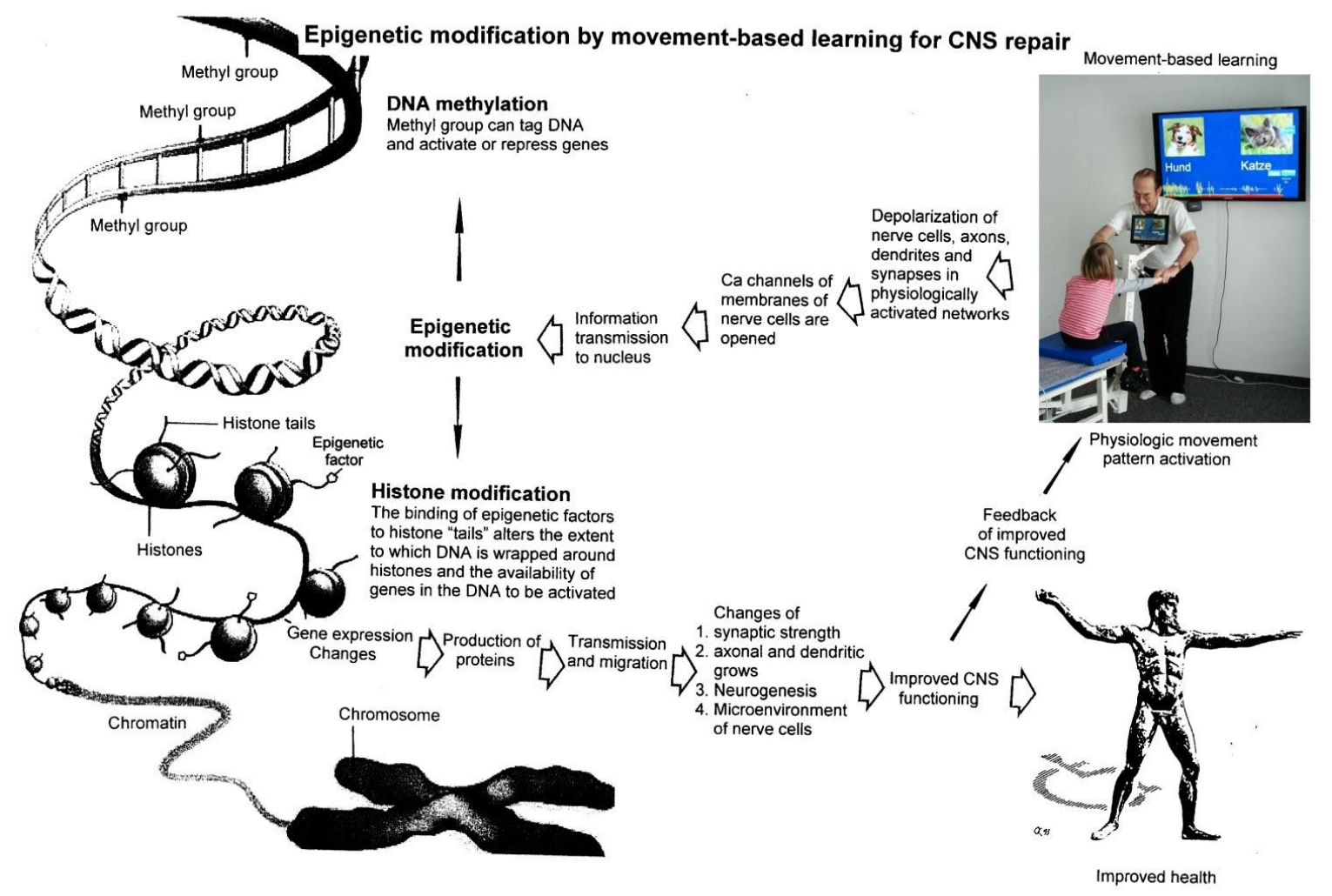

Figure 45. Epigenetic regulation for repair by movement-based learning. CDT-induced stimulation of the pathways that regulate neural network repair is a proven therapeutic and preventive tool. Epigenetic mechanisms, stimulated by physiologic network activation, are likely key players within signaling networks, as DNA methylation, chromatin remodeling and small noncoding RNAs superfamily are required for the fine-tuning and coordination of gene expression during neural network repair by learning. Since the nervous system is involved in nearly all body functions, CDT will improve health

likely the remained tract fibers were used already optimally. In Nefeli, approximately $30 \%$ of the cord remained following the injury.

In spinal cord injury generally, the coordinated arm, leg and trunk movements, performed when exercising on the special CDT device (Figure 1), induce exactly coordinated afferent input to the intumescentia cervicalis and lumbosacralis and between them. Since the neurons in the intumescentia cervicalis and lumbosacralis (Figure 46) are also coordination detectors, the thresholds for excitation are reached earlier (with less input), which means that the communication of the remained tract fibers following the spinal cord injury are used more efficiently and contributed to the repair.

Still some further progress was achieved in Nefeli. She became able to ride a normal bicycle with some problems and she liked it to. To become able to ride a normal bicycle helps SCI patients to become independent. The progress in learning to ride a normal bicycle is shown in (Figure 50).

With the improvement of the trunk stability, Nefeli (Figure 50A) became able to ride a bicycle with three wheels and fixed feet (Figure 50B). Since also other children used that three-wheel-bicycle (not shown in Figure 46) it was more a play with other healthy children than a balance training. Figure 50C shows that she really liked it to be on the three-wheel bicycle. With further improvement in the short-term memory Nefeli became able to use a four-wheel bicycle without the feet fixed (Figure 50G). Then she became able to use a normal bicycle with support of the father (Figure 50E). During playing Nefeli trained to improve movement patterns and balance (Figure 50G). Figure 50F emphasizes once more that she really enjoyed the playing/riding with the different bicycles together with healthy children. In Figure 50F the feet are not fixed as in Figure 50C.

Health improvement during healthy development by 'correction en route': Healthy children improve their CNS functioning during development by movement-based learning. Such movements include running, jumping and training balance. But because of fast food and iPhone mania, children often get overweight and move too little. Small mistakes made during development cannot be 'corrected en route' sufficiently through walking, running, jumping and other movements. Therefore, new diseases are expected to appear during childhood. To counteract the unhealthy development, children should exercise on the special CDT device to improve and support the normal development.

Figure 51 shows the human neural development, quantified by the low-load coordination dynamics values [4]. With age the CNS functioning improves; the low-load CD values get smaller (better). Exercising on the special CDT device improves the normal development, because the $\mathrm{CD}$ values get faster lower as shown in (Figure 51).

Figure 52 shows how to motivate healthy children to exercise on the special CDT device. When seeing a film together, they can exercise simultaneously on a special CDT device. In this case a friend, the sister and the brother of the patient Nefeli watch a film together on the tablet of Nefeli's device and having the sound by coupled air-phones (Figure 52). Still, the overuse of smart phones and tablets is big problem for healthy and CNS injured children and nowadays even of adults. 


\section{Spinal cord and brain stem injury repair}

Case report till CDT was started five years after the accident: When jumping on a trampoline, the eight-year-old Rafaela fell on the back and hit probably with the neck the metal support surrounding. She got up and went to toilette and vomited. When coming back she felt pain in the back and got dizzy. Her sister put her on a chair. The last what Rafaela said before losing consciousness was that she could not move the arms and hands any more. What really happened during the accident is unclear because Rafaela was alone and lost the memory around the accident (retrograde amnesia). After losing consciousness she was brought to a regional hospital. Four hours later she was sent to a university clinic. Her breathing became unregularly, and she was connected to a respirator. In the university clinic she was put to bed and the stuff waited that she recovers from the coma spontaneously! Being in coma for two days without recovery an MRI was made. As can be seen from the MRI, Rafaela had suffered in the meantime an incomplete injury of the spinal cord extending from the thoracic segments Th1/2 rostral till to the medulla oblongata (Figure 53B). All the cervical spinal cord segments were damaged probably by pressure, caused by the edema and/or lack of blood supply caused by the pressure.

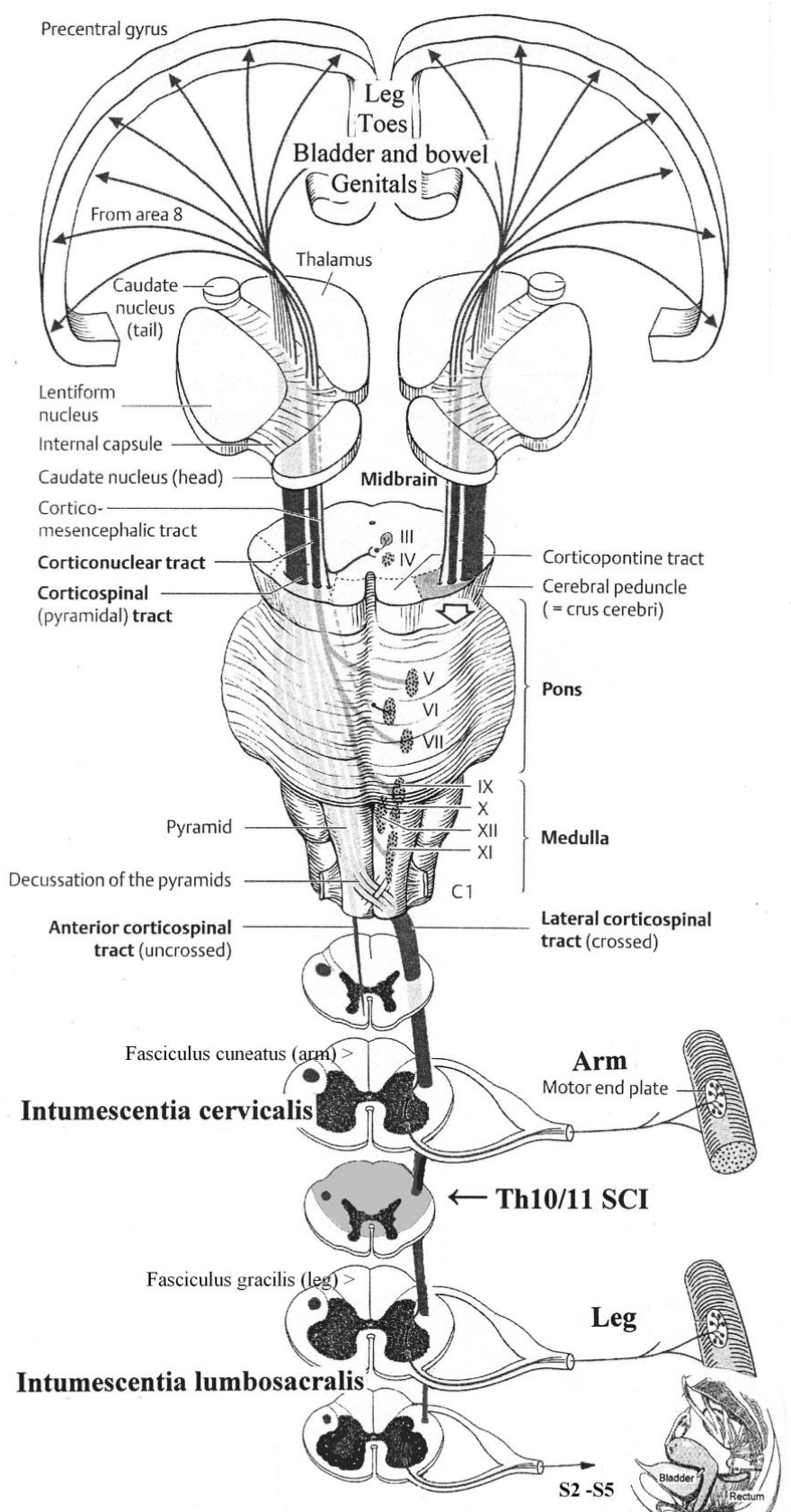

Ext. bladder and anal sphincters Genitals

Figure 46. The whole CNS is involved in the repair of the spinal cord injury Th10/11. Note, leg, toes, urinary bladder, bowel and sexual functions are closely related in the sensory-motor cortex 


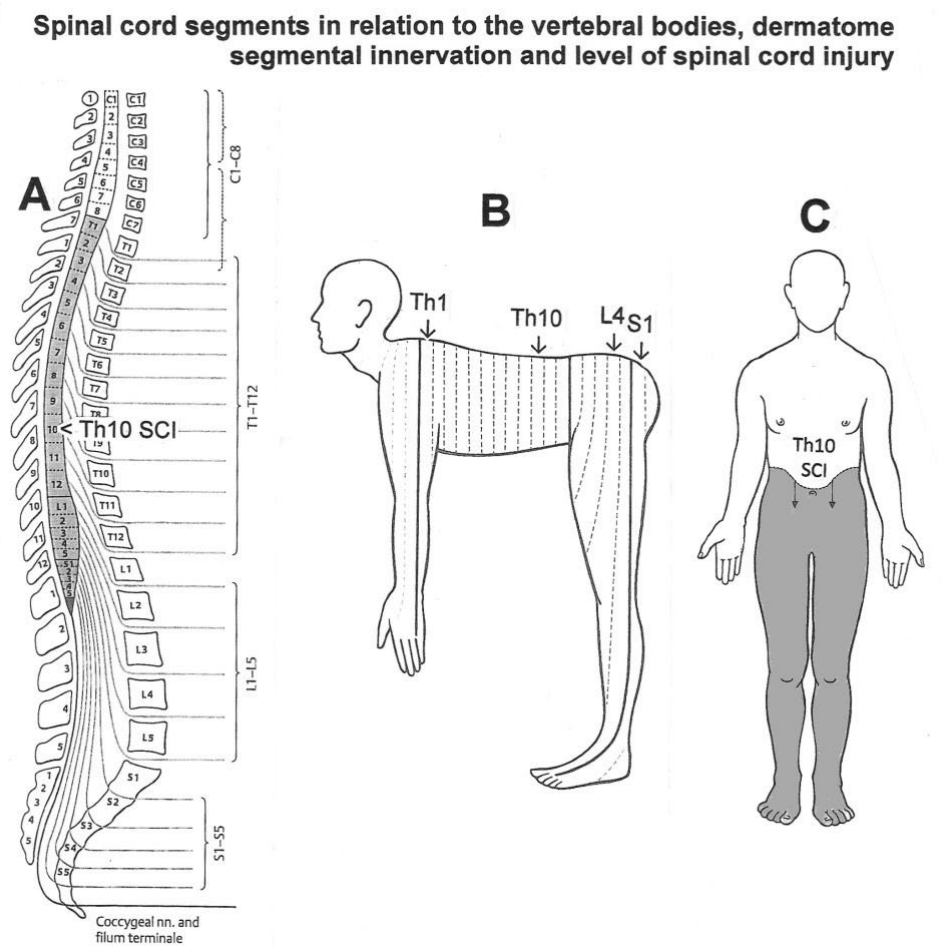

Figure 47 A. Spinal cord segments and their relation to the vertebral bodies. Note the Ascensus of the spinal cord, giving rise to the long cauda equina nerve roots. B. Segmental innervation of the skin. C. Below the spinal cord injury level there is loss of sensitivity and loss of connectivity to muscle and other functions
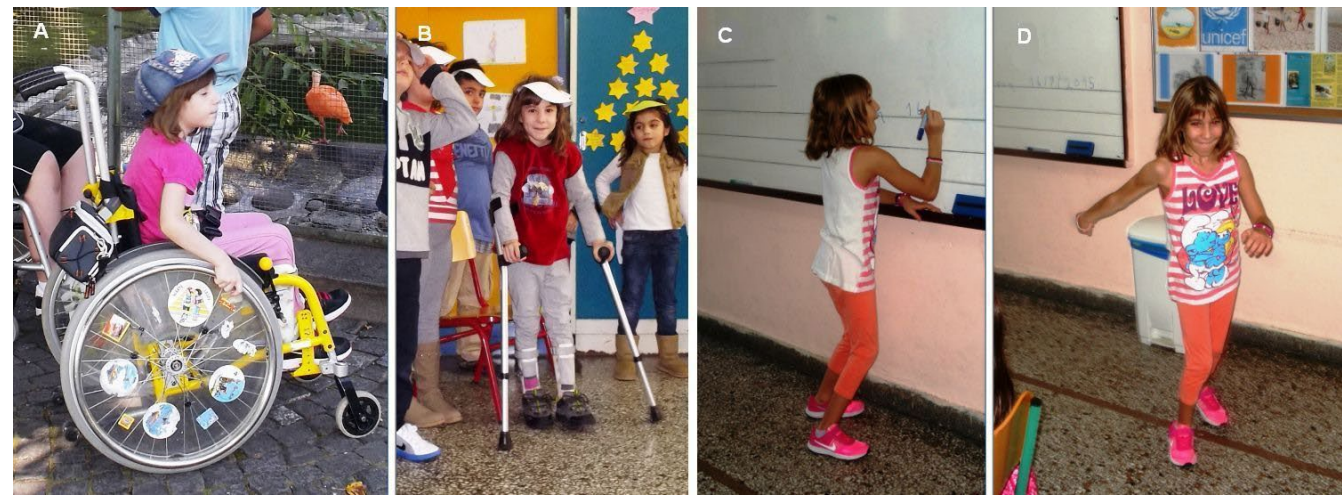

Figure 48. A. The 5.5-year-old Nefeli after suffering an incomplete spinal cord injury Th10 by medical malpractice. B. Nefeli after eight months of conventional children rehabilitation in Switzerland (Affoltern). Sticks and orthosis were needed. C,D. Ten-year-old patient Nefeli after six months of coordination dynamics therapy. At school she can walk again and can write at the white board

It seems that the injury included also a damage of the caudal part of the reticular formation and the nuclei of the vagus $(\mathrm{X})$ and accessory nerve (XI). The caudal reticular formation is the nuclear area for inspiration and expiration. This conclusion is supported by the clinics. Rafaela, being in the coma, had problems with breathing and was connected to a respirator. Five years later, Rafaela was operated because she had stones in the kidneys and a big stone in the bladder, indicating that, most likely, also the vagus nerve/nucleus was damaged, because the vagus nerve regulates the secretion of the kidneys. From Figure 54A it can be seen that the shoulders of Rafaela are too much curved forwardly. Obviously, the accessory nerve/nucleus, innervating the sternocleidomastoid and the trapezius muscles (Figure 53A), were also damaged.

When the coma regressed in Rafaela 10 days after the accident, she had no motor functions below the injury level. The physicians told the mother that Rafaela will stay like this for the rest of her life, misunderstanding that Rafaela was now in the phase of a spinal shock following the spinal cord injury. When after approximately three weeks the spinal shock regressed, some functions returned in her body spontaneously. Two months later the respirator was not needed any more. The breathing center of the reticular formation seemed to have partly repaired itself spontaneously. When in a dog the breathing center was damaged, it repaired itself within a few hours $[62,63]$.

After 3.5 months Rafaela was sent home. She could not walk, had little power in the arms (Figure 53A), was incontinent and could not speak. Her higher mental functions were not impaired, even though a small hypoxic injury cannot be excluded. One year after the accident she was treated in a rehabilitation center in Switzerland (where the Author was previously the head of the research department) with no or little progress. The time period of spontaneous recovery from an incomplete spinal cord injury is approximately one year. When Rafaela was sent to the Swiss rehabilitation center, the spontaneous recovery period 


\section{Neuron as a coincidence detector}
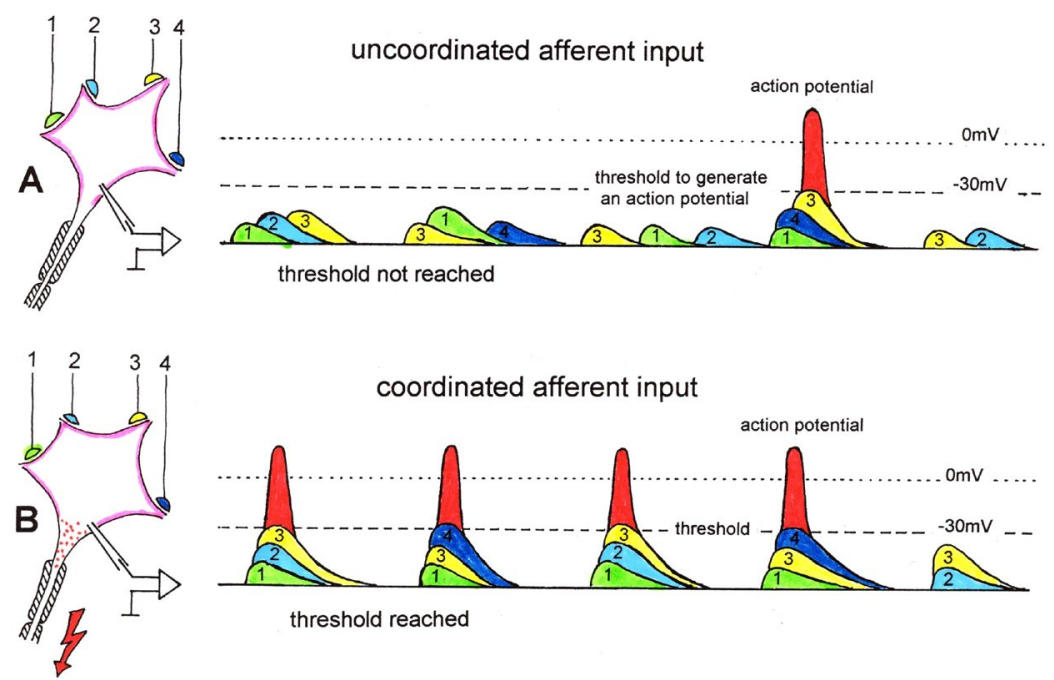

threshold reached

Figure 49. Neuron operating as a coincidence or coordination detector. A. Afferent input is reaching rather uncoordinated the cell soma. Only sometimes an action potential is generated, because the threshold of action potential generation is mostly not achieved. B. The action potentials in fibers 1 through 4 are reaching time-coordinated the dendrites or the cell soma. The postsynaptic potentials add up and the threshold is achieved at approximately $-30 \mathrm{mV}$, and action potentials are generated time-coordinated at the axon hillock. In the real CNS mostly, many smaller postsynaptic potentials will contribute to the generation of an action potential and passive conduction from the dendrites to the cell soma has to be taken into account. Coordinated afferent input may thus induce or enhance (coordinated) communication between neuronal network parts following CNS injury
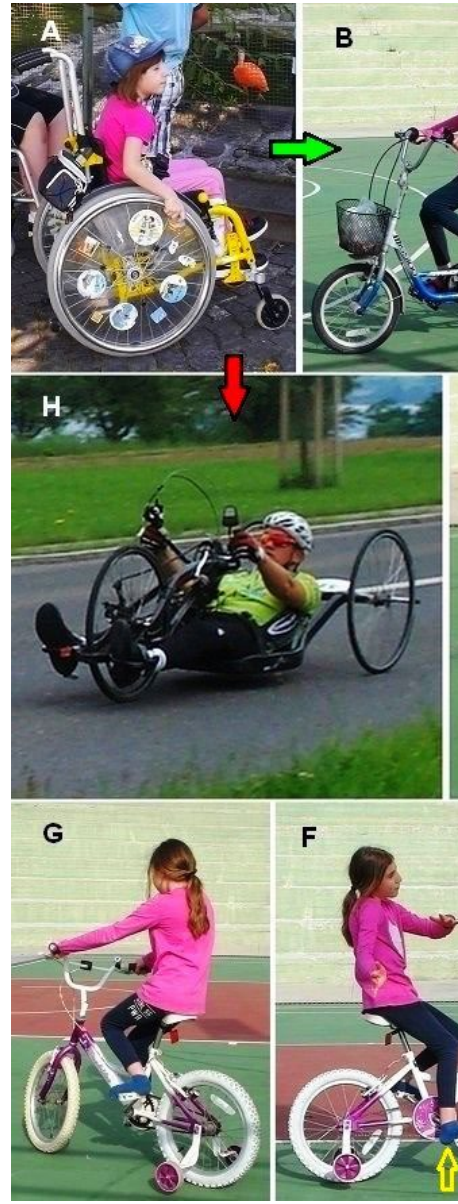
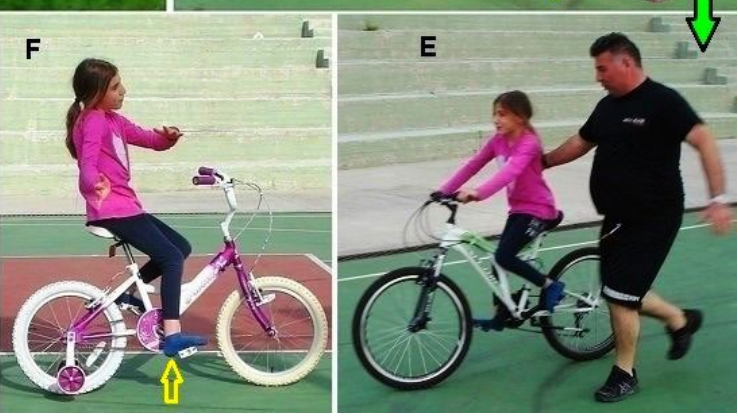

Figure 50. Relearning to ride a bicycle (A-G) instead of riding wheel instruments in the lying position (H) of patients with spinal cord injury. In B the feet are fixed. In C Nefeli is demonstrating the improvement of trunk stability. In D through G the feet are not fixed. E. With support of the father, Nefeli can manage a bit to ride a normal bicycle 
was already over, and no progress was achieved with the inefficient treatment. The mother of Rafaela was not informed about CDT, even though plenty of theoretical and practical medical research had been published in the time period, when the Author was the head of the research department in that Swiss rehabilitation center. for example, $[14,33,34]$.

Based on the thinking and knowledge that it is impossible to partly repair the human CNS, an orthopedic surgeon from USA made some muscle transpositions in Rafaela to improve the remained arm and leg functions. Such operations complicate CNS repair by movement-based learning and are in most cases contraindicated.

When Rafaela came for consultation to the Author five years after the accident (informed by the father of Nefeli about CDT), she was psychologically in a very poor condition as can be seen from the expression of her face (Figure 54A). She could not walk and was afraid of trying out the special CDT device. She could not persuade to try out the sky-walker. She could stand when leaning against the wall and she was able to write with the right hand. With the mother she managed physically at school and she learned well. But school knowledge is not helping much in later life, if she is fully dependent on the mother (Figure 54). Independence is the first big goal to be achieved with treatment. The mother tried to exercise her (passively) on the special CDT device in the sitting position which was not very successful but helped a bit. When Rafaela got her own sponsored special CDT device for training in the lying and sitting position, it turned out that in the lying position, when help muscles can be used for exercising, Rafaela could exercise by herself and her self-confidence improved strongly, and she wanted now to fight for a better future. This was the beginning of CDT.

From the point of view of injury severance, Rafaela has a good prognosis. She can become much better with respect to motor functions and she is good at school. The question remains whether optimal CDT

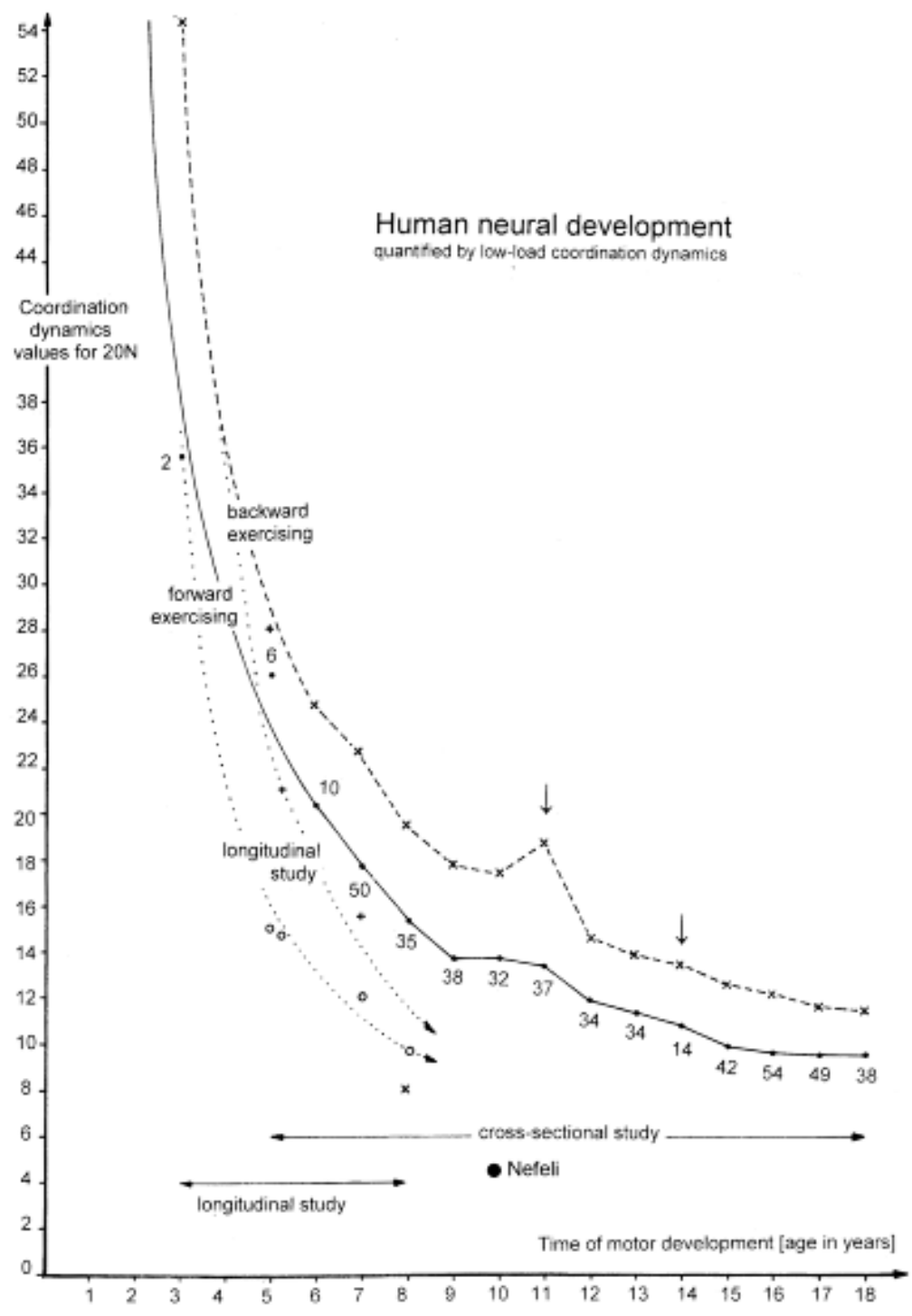

Figure 51. Low-load coordination dynamics values of boys and girls (lumped together) to quantify human neural development. Note that the coordination dynamics values transiently increase at 11 years and that the group size (the number) is small at an age of 14 (puberty, missing motivation to get measured), as is indicated by arrows. Note further that the coordination dynamics (CD) values for backward exercising during the whole developmental period are worse (higher) and that in the longitudinal study (dotted lines) CD values become smaller (better) than the average ones of the cross-sectional study due to the repeated assessment. The patient Nefeli with a SCI at the level of Th10/11 has a much better (smaller) CD value because of the performed CDT 


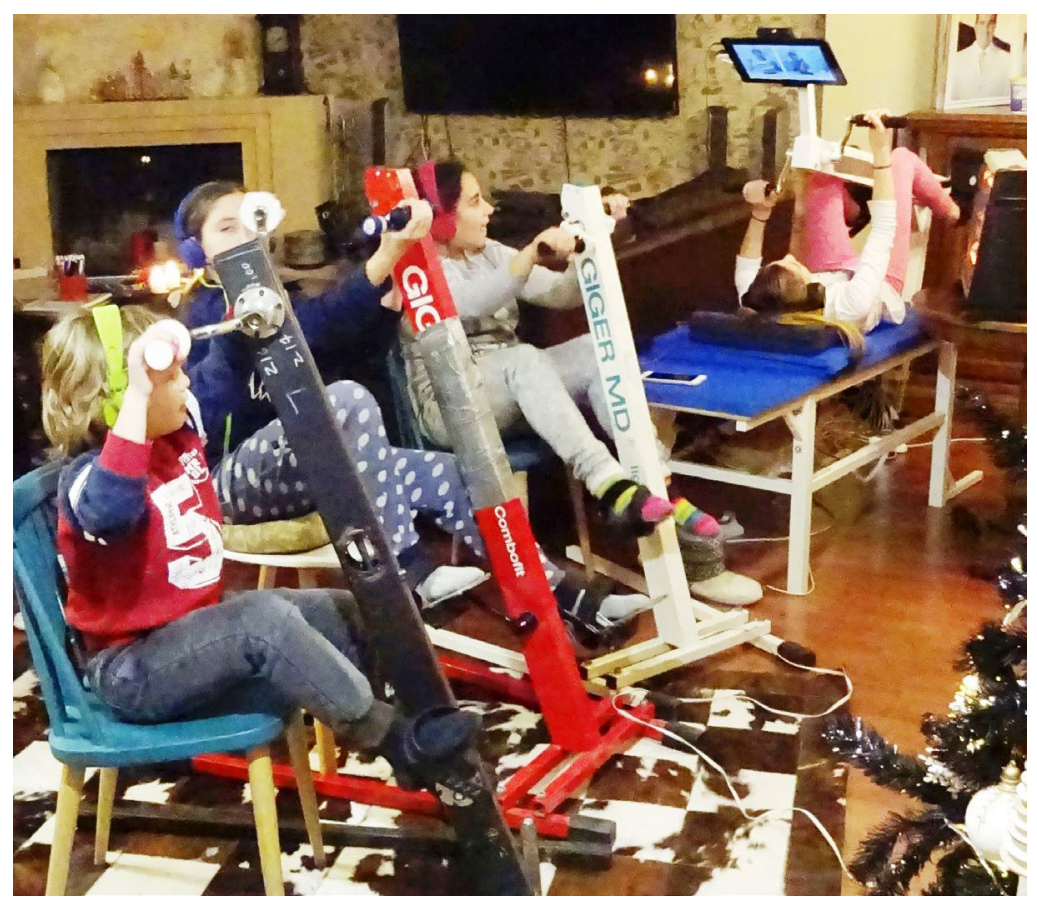

Figure 52. The patient Nefeli (SCI) training together with a friend and sister and brother on special CDT devices and watching the same film, which Nefeli is normally seeing during treatment to enhance the motivation for training
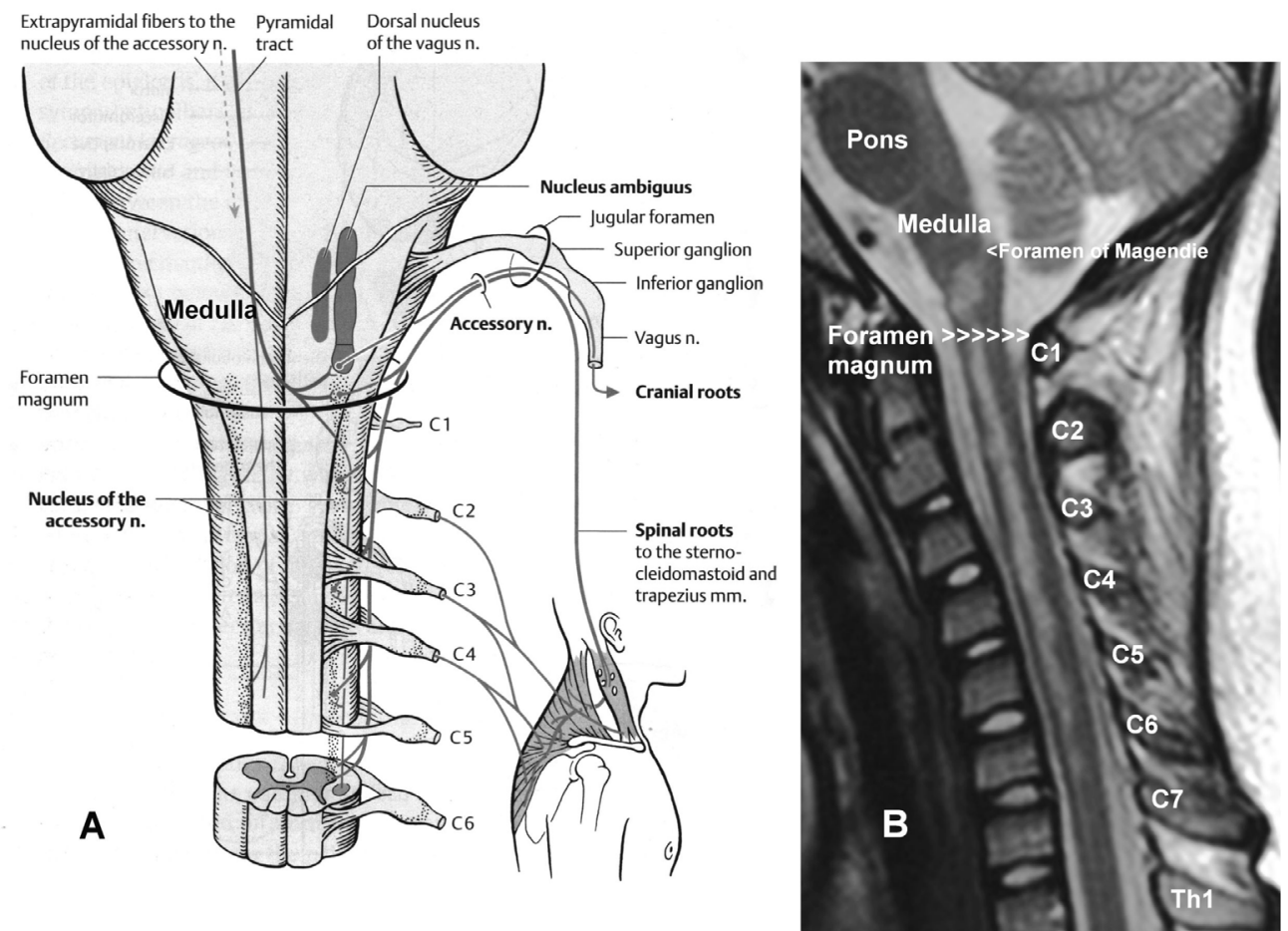

Figure 53. Comparison between a spinal cord/medulla injury, quantified by an MRI (B), and a picture of the anatomy of the spinal cord and the medulla (A). The trapezius and the sternocleidomastoid muscles are innervated by the accessory nerve (A). The somas of the motoneurons of the accessory nerve are damaged (B). Also, the nucleus ambiguous (X) was probably damaged (A). The dorsal nucleus of the vagus nerve (X) (A) is probably not damaged because it is located more dorsally in the medulla. Parts of the Figure from [64] 
can be administered to her for longer periods of time. A sponsor argued that Rafaela got worse in the last years when conventional therapy was administered to her.

Anatomy: Structural damage caused by the edema following the accident: According to the MRI of Figure 53B, Rafaela suffered an injury of the spinal cord and the caudal brain stem. The injury extended from the thoracic spinal cord segments Th1/Th2 rostral through all cervical segments to the caudal part of the medulla oblongata. A detailed analysis of Rafaela's spinal cord and medulla injury is important if one wants to understand why certain functions are lost or impaired, to adapt the therapy to the injury and to learn what structures can be repaired by movement-based learning.

The breathing of Rafaela was impaired at the breathing center (mainly inspiration), at the intercostal nerves and at the phrenic nerve levels. Looking at the MRI (Figure 53B), it can be seen that the caudal part of the breathing center was most likely damaged. When disconnected from the respirator she breathed only once and stopped then breathing. The rhythmicity of breathing was lost. But also, the motoneuron somas of the first two intercostal nerves were damaged according to the MRI. Looking at her breathing movements five years after the accident, it could be seen that the first two intercostal nerves were not fully working, because the ribs were not moving properly. Further, the phrenic nerves, activating the diaphragm, were probably also not working fully, because they arise from the damaged cervical cord segments C3 to C6, which were damaged. Looking at her breathing movements, it was not normal and also not a fully a paroxysmal breathing. Paroxysmal breathing is the breathing with only the diaphragm, typical for patients with a cervical spinal cord injury at the level C5/6. The thorax (rib cage) is getting smaller instead of increasing with inspiration and the stomach is coming up. Lucky that Rafaela could survive with this complicated injury and could breathe sufficiently by herself again after two months, when the caudal breathing center became repaired partly spontaneously. The rhythmic breathing had returned. The rhythmic firing of the network oscillators had repaired itself, probably stimulated by the rhythmic ventilation of the respirator.

After the injury, Rafaela could not speak. When CDT was started five years after the accident, she could speak but only quietly. The power for loudly speaking was absent.

Because the nucleus of the vagus nerve was damaged (Figure $53 \mathrm{~A})$, she got repeatedly stones in the kidneys. The motoneurons of the accessory nerve, arising from the $\mathrm{C} 2$ to $\mathrm{C} 4$ segments (Figure 53A), were also damaged because the trapezius muscle was activated too little (Figure 54A). The functions of the glossopharyngeal nerve seemed not to be impaired.

The main functions to be improved were therefore walking, arm and hand movements, breathing and the autonomic functions to improve for example the kidney function and re-establish full urinary bladder continence. Kidney functions and breathing are essential for life. With the shallow breathing a lung infection is at risk.

CDT repairs by coordinated arm, leg and trunk movement's not only somatic functions, but also by learning transfers the autonomic functions. If damaged CNS parts or nerves are included more directly in the movement-based learning, then most likely, the efficacy of repair is higher. In this patient the trapezius and sternocleidomastoid muscles were trained more directly when the patient performed strong rotational movements on the special CDT device.

But how can the vagus nerve trained more directly? The urinary bladder can be trained more directly when jumping on springboard. But in this way the sacral parasympathetic nervous system is mainly stimulated and not the cranial portion. We are left for the time being with the exercising on the special CDT device, which activates the whole parasympathetic division by learning transfer.

The breathing center of the brain stem can be repaired more directly when pushing the patient to her limits by increasing the load (Newton). The necessary deeper breathing will activate more strongly the breathing center in the reticular formation to improve its efficacy. The hematocrit will also increase with a deeper breathing, which is good before an operation not to need blood from other persons.

Medical malpractice in Rafaela: If following a head or neck accident the patient loses consciousness, then there are dangerous pathologic processes in progress as bleeding, edema or embolic infarction.

Standard medicine would be to make an MRI, which would reveal ischemia within a few minutes. Diffusion-weighted MRI detects ischemia highly reliable anywhere in the brain. Digital subtraction angiography (DSA) (i.e., visualization of the blood vessels) provides the best morphological view of pathologic changes in the extracranial and intracranial vessels. Even ultrasound techniques would show that brain tissue damage is in progress. But just putting the patient in the hospital into bed and waiting that she recovers from coma is not standard medicine. "Lucky that it was recognized in the
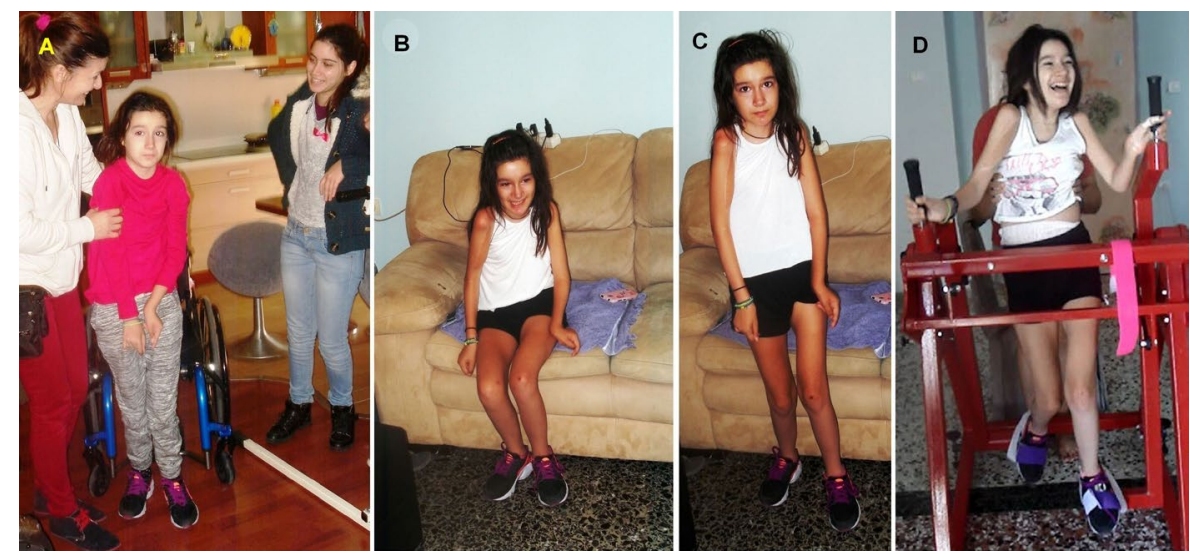

Figure 54. A. The patient Rafaela (with mother and sister) with an incomplete spinal cord injury extending from the thoracic Th1/2 segments rostrally to the medulla oblongata (including all cervical segments). Note the bending of the shoulder and the hanging of the arms, due to the injury. The expression of her face characterizes her psychological state. B,C. Through CDT, Rafaela succeeded to get up from the sofa by herself. D. She became able to perform sky-walking following three months of therapy 
university clinic that the breathing of Rafaela arrested and that she was connected to a respirator". Medical malpractice of such extent cannot be compensated for by the best brain repair treatments, if available, and is not in accordance with the opinion of Hippocrates.

Stage of repair five years after the accident when CDT was started: When coordination dynamics therapy (CDT) was started, she could just exercise by herself on the special CDT device in the recumbent position, which is the first movement such a patient can do by herself. She could perform one or two steps alone before losing balance. She could not exercise in the sitting position and the coordination dynamics, the CNS functioning, could not be measured. She was still incontinent. She could speak but quietly. But she could write and use the iPhone with the right hand.

Improvement of CNS functioning through 3 months of coordination dynamics therapy: After three months of CDT she could stand up by herself from the sofa with a few trials (Figure $50 \mathrm{~B}$, C) and stand and could exercise in the sitting position, so that CNS functioning, by means of the coordination dynamics (CD) values could be measured.

When comparing Rafaela's face expression after three months of CDT (Figure 54D) with those before the therapy (Figure 54A), it is obvious that her mental state had improved. She laughed when she managed to exercise on the sky-walker in the standing position (Figure 54D) and also when the Author exercised with her. The positioning of the shoulder (trapezius muscle) and of arms and hands improved a bit (Figure $54 \mathrm{C}$ ), in comparison to before starting CDT (Figure 54A). Urinary bladder functions improved, but the repair was not fully achieved. She still had only a few minutes from the first desire to void to spontaneous micturition. She became able to manage better at school. Rafaela has a good prognosis for further CNS repair if her therapy is continued aggressively, because her severe CNS injury was balanced, that means all functions were working a bit.

\section{Cerebellum repair}

Cerebral palsy: Sophie had from birth an atrophy of the cerebellum and ponds (cerebral palsy) [61]. At an age of 5.5 CDT was started. At the beginning Sophie could not perform any movement accurately. She was incontinent and her speech was poor. The Author could not understand her. She could crawl on the floor in trot gait coordination with poor performance. Pace gait crawling was not possible.

Sophie could not jump because she did not have that pattern learned and stored in her CNS and jumping is no automatism. She learned to jump and hated it first. At the beginning, she could jump a little by herself on a trampoline. The jumping on springboard was harder to learn. The supported jumping was a big load for the parents, because Sophie contributed only little. Slowly she contributed more and more and at the end she could jump by herself on springboard but with poor performance. When she had learned the jumping, she liked it.

Of course, Sophie could not walk. When the mother tried to walk with her, she was more hanging in her hand than walking (Figure 55A, B). There was no real walking pattern and no balance. She could not really learn it, because she had no protection automatisms when falling, which are probably normally stored in the cerebellum. If she would fall, she would injure herself and that is what the parents were very afraid of.

The first fundamental progress in repair was achieved when the protection automatisms appeared in Sophie at an age of 6.5 years. The parents were not so much afraid anymore when trying to make her walking and Sophie tried now to walk by herself. The protection automatisms became operationally at a time when she became able to exercise by herself on the special CDT device.

When the protection automatism occurred, the learning of walking became much easier and less risky. Sophie was not afraid to fall anymore and tried herself to walk without falling and she liked it (Figure 55). At an age of 8 she was able to walk without falling (Figure 55C). Sophie's trot gait crawling improved. The pace gait crawling became possible with poor performance.

At an age of 9, Sophie could creep (Figure 2), crawl (Figure 3A), walk, jump and play a bit with the ball. She was fully continent. Her speech had improved, so that the Author started to understand her, but was still far away from normal. The coordination of the speaking with coordinated movement is helpful (Figure 56), since deficient speech is mainly caused by insufficient speech muscle coordination caused by cerebellum and pons atrophy.

She had still problems with the balance, but she managed now without falling. Her writing got better. Altogether, her higher mental functions improved. But she is not able to run so far. To generate the high frequency seems to be the main problem. Probably she will learn it with ongoing therapy.

Traumatic injury of the cerebellum and pons: The 55-yearold Dr. Cwienk suffered a very severe traumatic cerebellum injury and a severe brain injury. Figure 57A shows the intelligent patient Dr. Cwienk before his severe brain injury. His face shows not only a friendly expression, but also some power of intelligence. After suffering the severe brain injury (Figure 57) and partly recovering from it during 10 years of CDT, he shows in Figure 57I a friendly face again, but the power of intelligence is missing. He himself used to say: "Before the injury I could do two things at the same time, but now only one". Therefore, the mental and emotional states of a patient can partly be seen in the patient's face expression. In coma patients (see below) we may judge a bit from the facial expression, in what state the patient's nervous system is in and get some additional information for the prognosis of the outcome. This is important, because we cannot ask the patient. The wife of Dr. Cwienk reported that her husband looked good when he was in a coma. Conventional treatment was only started three months after the accident. When the patient was out of the coma in Figure 53B-F his face did not look healthy. There are two possibilities which may have contributed to his state. Firstly, efficient movement-based learning was started too late with the consequence that the injured brain deteriorated further in the meantime. Secondly, the patient may have been in a good emotional state while he was in the coma.

The now 78 years old patient is performing CDT every day for 20 years to keep the level of health. But when he stops therapy for three days or more, he is going backwards physically and mentally as his wife said. For further see below under aging.

Comparison of therapy induced improvement of CNS functioning following cerebellum and pons atrophy and traumatic injury of the cerebellum and pons: A comparison of re-learned movements through CDT of a cerebellum and pons atrophy of the 9-year-old Sophie and after traumatic cerebellum injury of the approximately 65-year-old Dr. Cwienk shows similarities and differences $[15,65,66]$. Even though Dr. Cwienk lost approximately $70 \%$ of the cerebellum and had an additional brain injury, still the comparison is interesting. 
1. The most important difference between the now 9-year-old Sophie and the at that time 65 -year-old Dr. Cwienk was the speed of learning, when CDT was administered. What Sophie learned in 12 months, Dr. Cwienk needed a few years.

2. Sophie learned the protection automatisms when falling within 6 months and it was easy to walk with her when holding her hand. Already the walking in interpersonal coordination with Nefeli (SCI) improved her walking performance (Figure 3C). Her left arm was abducted to keep balance.

Dr. Cwienk did not re-learn the protection automatisms, when falling, within 10 years of therapy. With a lot of concentration, he could move step by step without falling (Figure 3D). But when he was really falling it was nearly impossible to support him, because his reactions were un-physiologic and unexpected for the supporter. It was therefore stressed to support him during walking.
3. The performance of the walking pattern was smoothly and physiologic in Sophie (Figure 55C) apart from the balance problem and the inability to stop the leg movement on time (Figure 2), but not in Dr. Cwienk (Figure 3D).

4. Sophie could combine the getting up movement and the walking. Dr. Cwienk could not combine the two movements. He first had to get up, concentrate and then start to walk step by step.

5. Sophie could perform sky-walking, including sky-walking with crossed arms to activate commissural fibers. Quite often she overstretched the knees, what she was not doing during normal walking. This means that sky-walking is not an inborn automatism like walking. Later on, Sophie learned the sky-walking without overstretching the knees. Sky-walking was not administered to Dr. Cwienk. But since he could walk on treadmill with both hands and one hand, he could most likely also move on the sky-walker because

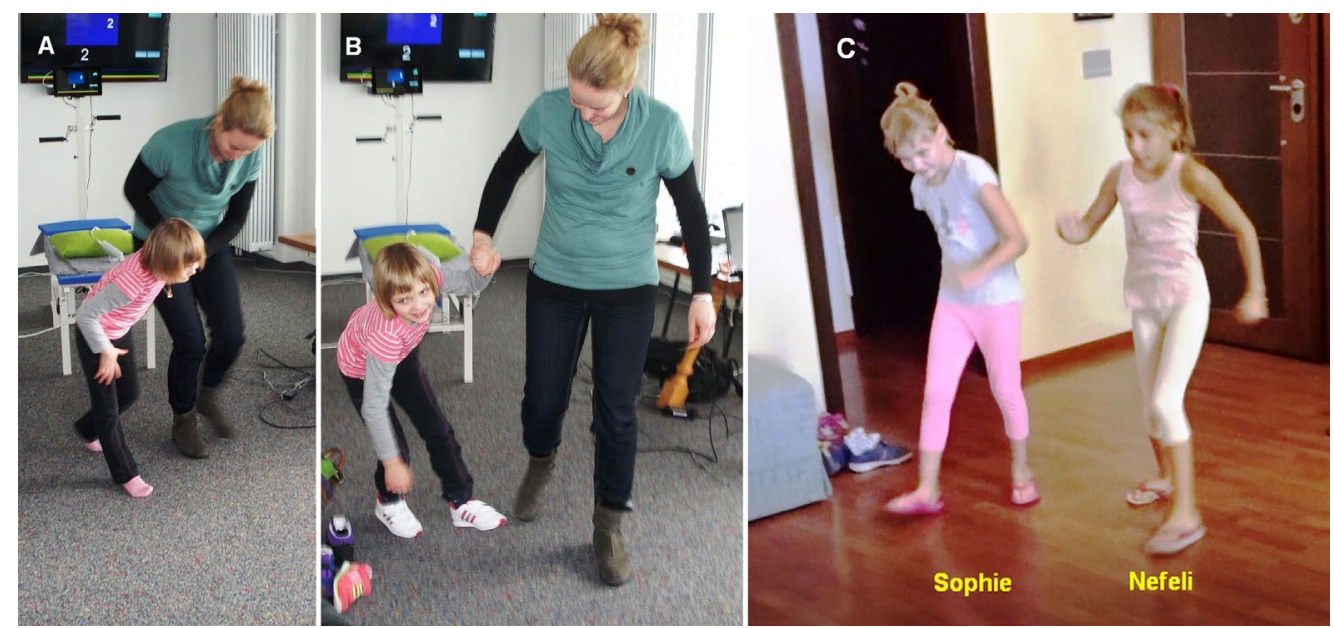

Figure 55. A, B. Sophie, the cerebral palsy girl with an atrophy of the cerebellum and pons, was unable to walk with her mother before CDT was started. She could not generate a walking pattern. Knees were overstretching (A) which blocked the walking pattern; she was immediately falling when trying to walk (B). C. Sophie (left) learned walking through CDT. When Sophie (cerebral palsy) and Nefeli (spinal cord injury) were training together (here walking), they motivated each other to fight more

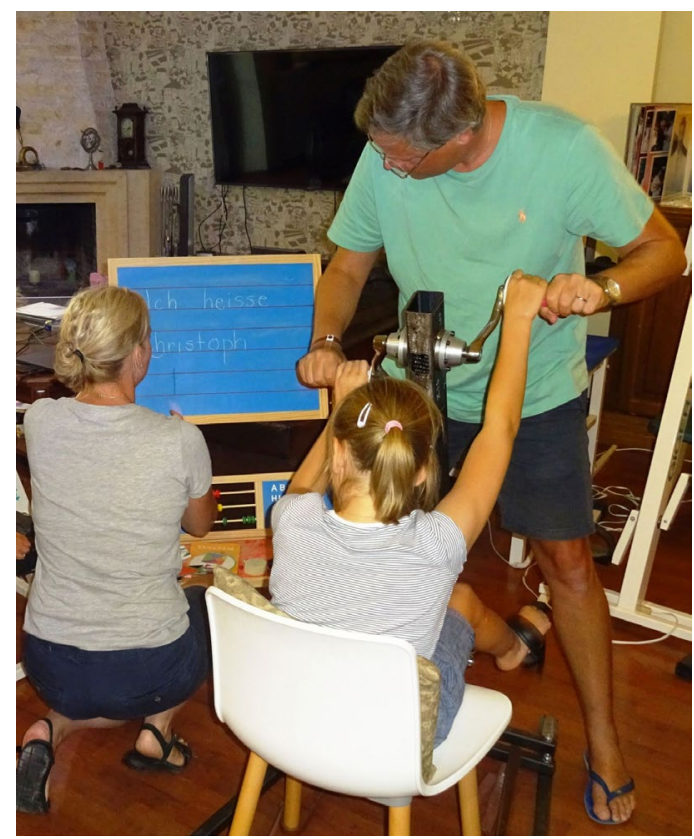

Figure 56. Sophie during speech therapy when exercising on a special CDT device with father and mother 
no balance is needed. Walking with sticks was already more difficult for him.

6. Sophie learned a bit the jumping on springboard with poor performance within 6 months. Dr. Cwienk re-learned the jumping on springboard, but the smoothness was missing. Because of extensor spasticity and missing elasticity, there was the danger of damaging the knee joints.

7. Sophie and Dr. Cwienk re-learned the trot gait crawling, even though the performance in Sophie was better. But the pace gait crawling was difficult for both of them. The problem of Dr. Cwienk with the pace gait crawling is understandable because of his one-sided cerebellum injury in some similarity to stroke. But Sophie had an atrophy of the cerebellum and not a one-sided injury. Also, the 10-year-old Nefeli (SCI) had more problems to re-learn the pace gait crawling (Figure $3 \mathrm{~A}$ ), even though during development the pace gait crawling is learned first because it is easier for the CNS to generate it.

8. When being able to exercise on the special CDT device, both, Sophie and Dr. Cwienk could exercise on it in the standing position (Figure 58 ), because only little balance is needed.

9. Sophie had to learn to become fully continent. Dr. Cwienk had no problems with urinary bladder functioning.

10. Sophie and Dr. Cwienk had scanning dysarthria and dysarthrophonia. Both patients had also a paravermian injury/ atrophy and an impaired coordination of the musculature of speech. Both patients were speaking slowly and haltingly with poor articulation. In both patients the speech improved with therapy but was still far away from physiologic after 12 months and 10 years of CDT respectively. To enhance the rate of learning/relearning of speaking, speech therapy was partly performed when the patients were simultaneously exercising on the special CDT device (Figure 59) to improve CDT functioning in the short-term memory in general and speech performance in specific.

11. The improvement of CNS functioning in the long-term memory improved the face expression strongly in Dr. Cwienk within 10 years (Figure 53). In Sophie the expression of the face became livelier.

12. Dr. Cwienk, being 75 years old, was performing approximately 10 hours CDT per week to keep the achieved level of repair. He had no recurrence of a colon carcinoma. Sophie continued intensive therapy. It seems that her prognosis to recover from the atrophy of the cerebellum and pons is good, may be in similarity to children with hydrocephalus were the prognosis of recovery is surprisingly good. Further therapy will show how much correction 'en route' of the development to repair the cerebellum atrophy is possible in Sophie.

13. In spite of the tremendous progress of the very intelligent Dr. Cwienk with CDT, he could not reach the intelligence level of
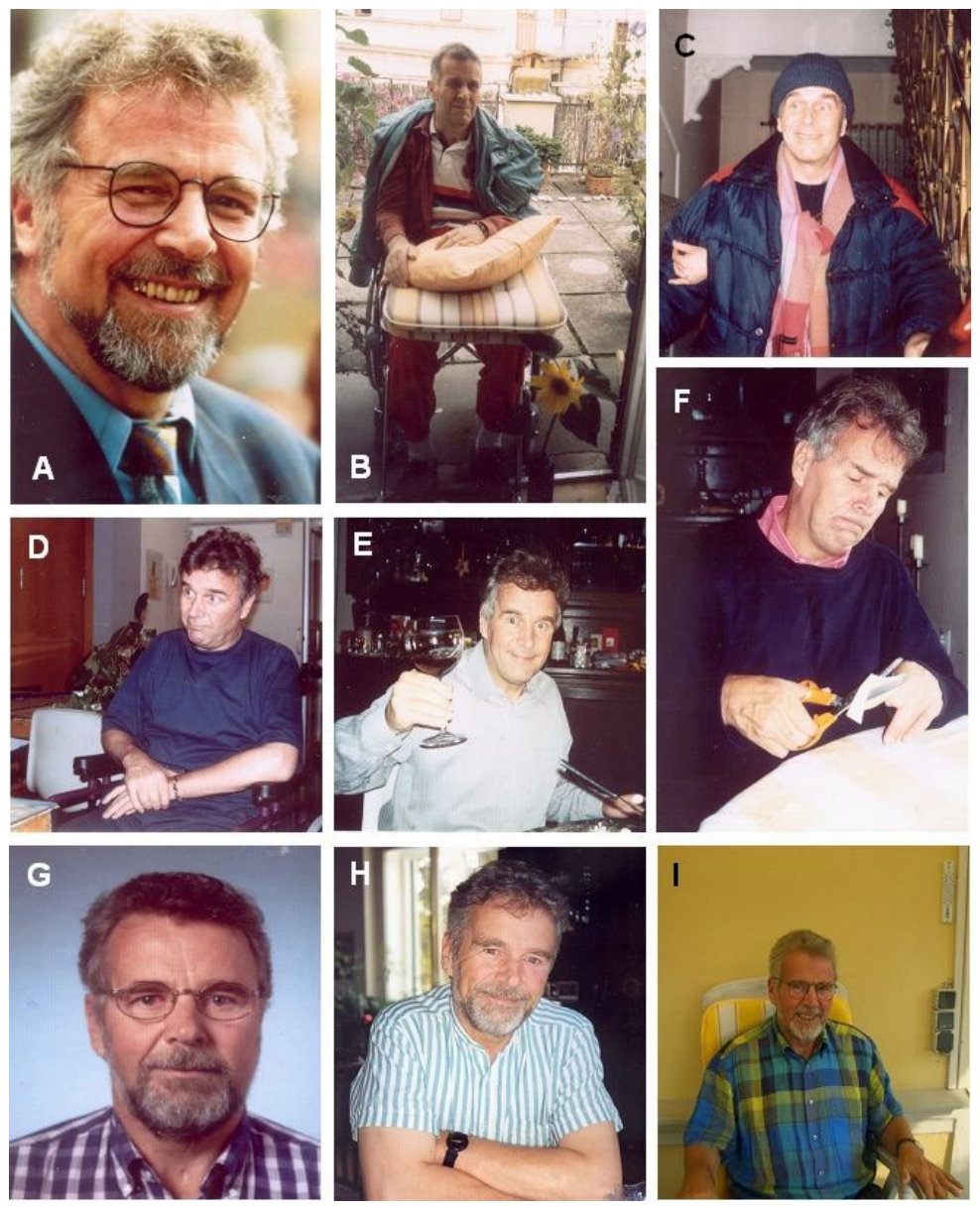

Figure 57. Therapy-related improvement of the facial impression of a patient who suffered severe cerebellar and cerebrum injuries. A. before the accident; B-I. after the accident in 1995 till 2006 
before the injury. As he said himself, before the injury he could listen to somebody and generate arguments against the opponent at the same time. Now, after the recovery from the severe brain and cerebellum injury, he can do only one thing at a time. This means, he first has to store the information and then develop a strategy against the arguments of the opponent. The memory seemed not to be impaired. The intelligence of Sophie improved with therapy.

14. Dr. Cwienk was an extremely cooperative patient, which helped him to recover partly from the severe cerebellum and frontal lobe injuries. The cooperation of Sophie was poor so far. The parents had to be very patience to administer therapy successfully. But when she learned a new movement, first time in her life, she used it immediately like crawling, walking on knees (Figure 3) or walking.

15. The writing and drawing was poor in both patients but improved. For some improvement of hand coordination Sophie needed 9 months and Dr. Cwienk a few years. After 2006 the handwriting of Dr. Cwienk got worse due to aging, reduced therapy and not writing with the hand anymore. The handwriting of Sophie improved with further therapy.

16. No changes in the MRIs with therapy could be seen in both for the atrophied cerebellum and the injured cerebellum. Repairs were mainly due to functional reorganizations.

17. At an age of 9.5 years, Sophie learned a bit to run through 4 years of CDT. Dr. Cwienk never had a chance to re-learn free running.

Brain injury repair in children: The three boys, aged nine, twelve and fourteen years old (Benjamin, Mario, and Andrej, respectively), suffered severe brain injuries almost at the same time, two in a car accident and one in a bicycle accident. In the nine- and fourteen-yearold patients, intensive coordination dynamics therapy (CDT) was started approximately five to ten weeks after the trauma in vigilant coma stage. The two patients recovered unexpectedly early from the coma and progressed quickly in their recovery of motor functions. They re-learned running after four months of therapy, even though their locomotor functions were still far from normal. The twelve-yearold patient Mario did not obtain intensive CDT, only conservative physiotherapy because of many infections and complicated bone fractures, which were not invasively reconstructed. The patient
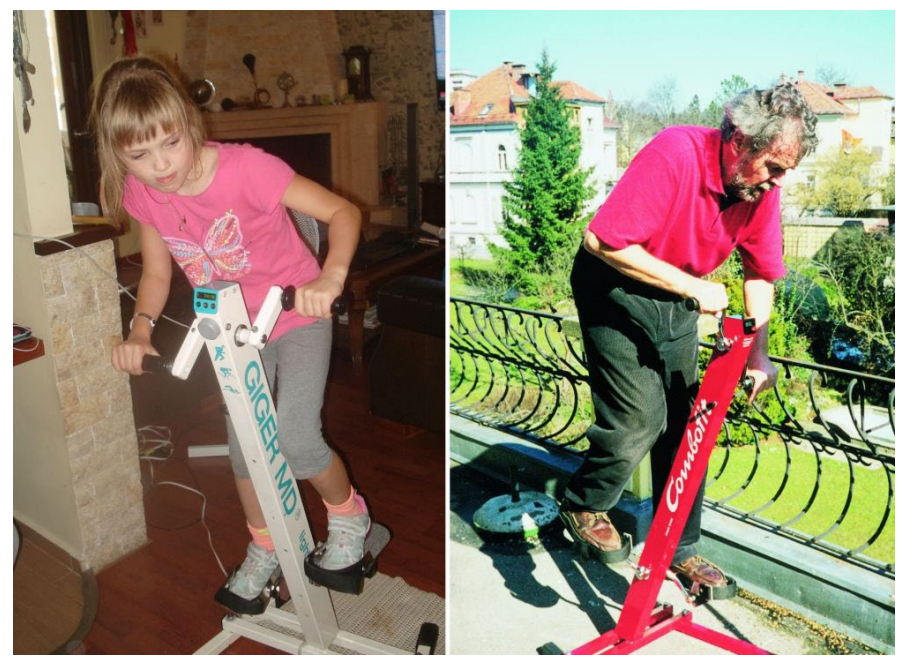

Figure 58. Exercising on the special CDT device in the standing position of the 7-yearold Sophie with cerebellum and pons atrophy and a 60-year-old patient with traumatic cerebellum and pons injury. Note that when no balance is needed both can perform this coordinated arm and leg movement, but the girl with more elegance recovered much later from the coma (six months as opposed to six weeks) and had severe extensor spasticity, shortened tendons, problems with several joints, pain, reduced mental functions, grasp reflex in the right hand (reappearance of infant automatism) and nearly no useful motor functions in his legs. Mario received intensive CDT following a delay of five months. After a few months of CDT administered to all three boys, Mario obtained treatment at home, Andrej got conventional therapy and only Benjamin was treated with CDT over 18 years. For details of the three cases see [3-5,67].

The outcome of the three patients by movement-based learning is compared in (Figure 60). Mario and Andree are living at parents' home as disabled adults because CDT was not administered to them for a few years. Only Benjamin obtained a rather optimal CDT and reached a meaningful life. He successfully finished a university study and won the bronze medal in 100m sprint in 2016 during the European Championships in Grosseto [61]. In 2019 he was even able to win a silver medal in a local competition among healthy athletes. Benjamin can really run fast (Figure 61C) and jump long distances.

These case reports demonstrate that in CNS injuries, efficient neuro-therapy by learning has to start as early as possible following the trauma to avoid pathologic reorganizations. The rate of re-learning reduces dramatically with the severance of the brain injury and the inefficiency of the treatment. Important is the long-term outcome and whether can they achieve a meaningful life? Urinary bladder problems only occur in extreme severe brain injuries.

In the section aging, the healthy ageing Author will compete with Benjamin and the following patient Sotiris, who suffered also a severe brain injury.

\section{Brain repair in adults}

A 23-year-old male engineering student suffered a severe brain injury in a car accident. He did not lose bladder continence. He needed to use a wheelchair and his higher mental functions, including speech, were impaired. The patient attended a rehabilitation center for eight months and was able to dispense with the wheelchair. Mainstream medicine had given up on him. The family found CDT on the internet and started it one year after the accident. At the beginning, he trained at least 20 hours per week and he later trained at the limit with up to 30 hours per week. When the Author's involvement was increased and the patient's sister, also an engineering student, started treating her brother, optimal CDT was started. The injury was balanced in that the brain injuries were evenly distributed. He could perform most movements to a small extent. The most significant obstacle to the resumption of a meaningful life seemed to be the impairment of the higher mental functions. The quality of the higher mental functions of this cooperative patient fluctuated. Motor functions and speech improved after three years of therapy. The ultimate goal of continuing his studies in engineering was achieved after 6 years of aggressive CDT in 2015. With the help of the sister and the university he was able to finish his study fully in 2019. The patient has still problems with the higher mental functions and the speech, but he easily drives a car. The sister said, he is driving the car in the same way as before the accident. When the Author was with him in the car, he could not complain about his driving. Car driving seems to be an old-learned pattern.

The great improvement of CNS functioning (lowering of the values) in the patient Sotiris while performing CDT [61] can nicely be seen by plotting the high-load CD values (Figure 30 ). The values decreased from 449 to approximately 60 , that means by a factor of 7 . The selforganization of CNS functioning improved substantially. It can also be 


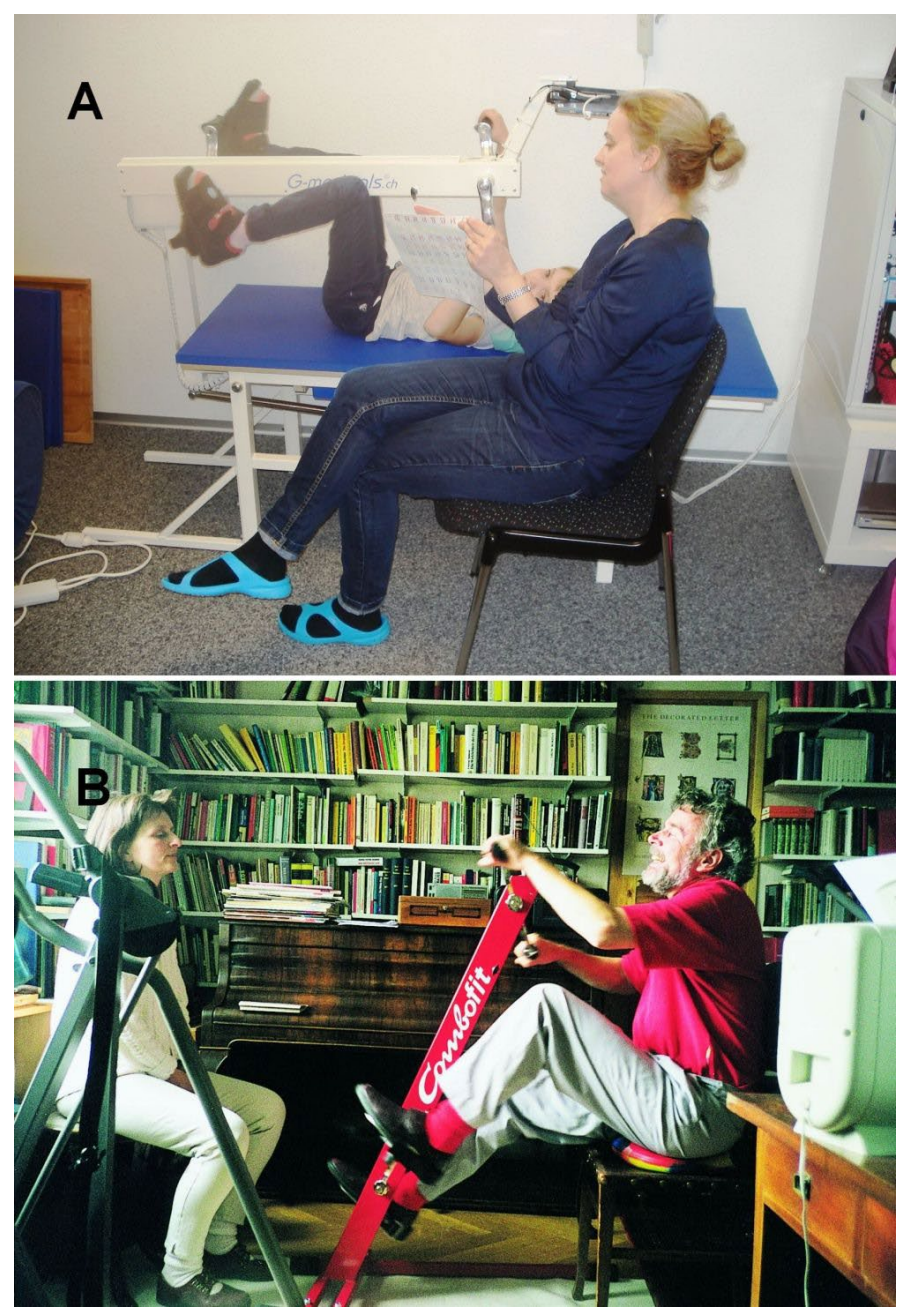

Figure 59. Speech therapy during exercising on the special CDT device in a girl with cerebellum and pons atrophy by the mother (A, teacher) and an adult patient with traumatic cerebellum and brain injury by a speech therapist (B)

seen from Figure 30 that the improvement of the forward-backward symmetry of exercising took 40 months. The healthy pupil learned this forward-backward symmetry in half a month [4]. The rate of learning for this forward-backward symmetry was reduced by a factor of 80 in this patient. In stroke patients, the repair of the right-left symmetry is an even greater problem.

From Figure 30A, it can further be seen that supervised intensive therapy was more efficient for repair than training without supervision.

Figure 30 also shows that the overall CNS repair needed longer than three years. The insets $B$ and $C$ of Figure 30 show the repair curves of a normal pupil (B) and an athlete pupil (C) adapted to the time scale. It is obvious that the healthy pupils learned faster. The $\mathrm{CD}$ value curves reduced quickly to the plateau, indicated by a dotted line. The overall performances of the movements were also better. The CD values for forward and backward exercising started from around 390 to 450 in the patient and those from the pupils from 160 to 200.

When the patient continued the study, he had to reduce the training times from 30 to 10 hours per week. His high-load coordination dynamics values got worse (higher). After finishing the study, he increased the training times up to 30 hours again and got much better again (under 50) (Figure 30D).
In this patient the improvement of pattern stability could be nicely shown in Figure 28. The improvement of the stability of the pattern running on treadmill needed approximately one year (Figure 28).

To bring a brain-injured patient fully back to life, it is important that he can work again. To work as an engineer, he has to be able to perform necessary skills apart from computer-driven machines. A test was undertaken to proof how good he can perform skills he had learned before the accident.

As found out, Sotiris was not able to file a plane surface or saw metal properly (Figure 62A). With the turning lathe, he was able to lathe (Figure 62B) and drill holes (Figure 62C). He was able to caliper with a slide gauge, having a vernier scale for measuring the $1 / 10$ of a $\mathrm{mm}$ (Figure 62D). To measure and to lathe was stored in his memory, but not to fix a rod in the lathe chuck. Altogether, Sotiris has a good chance to work again back in his old profession (Figure 62).

Since Sotiris could perform the old-learned movement 'car driving', but could not file a plane surface, it seems that Sotiris never really learned the proper filing. This means that really learned patterns during development or later is a special quality of brain functioning, which can be used later on for brain repair if necessary. The learning of movements and skills is important for the development of a good

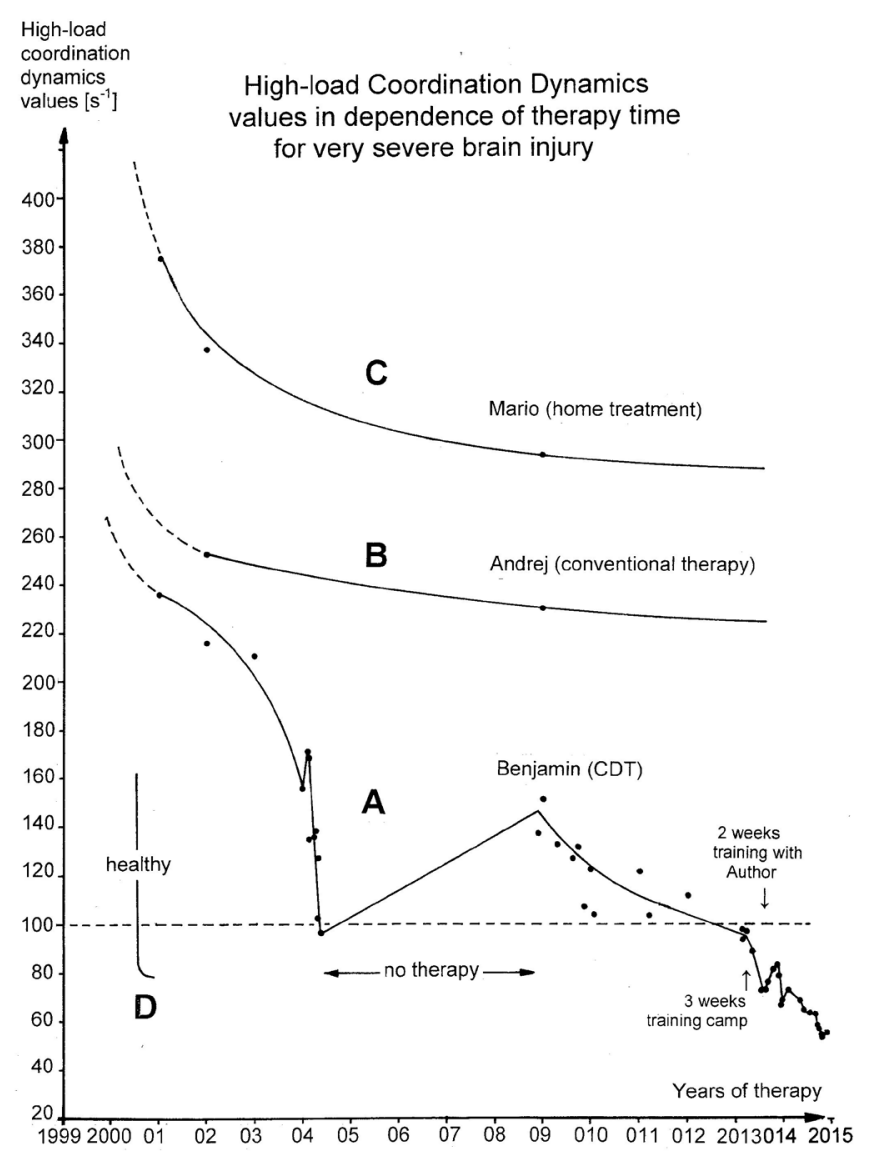

Figure 60. High-load coordination dynamics $(C D)$ values in dependence of therapy in the three patients with severe brain injury Benjamin (A) (CDT), Mario (B) (home training) and Andrej (D) (conventional therapy). The improvement of high-load CD values of healthy pupils upon repeated exercising on the special CDT device are inserted according to scale (D). Note that the high-load CD values of Mario and Andrej are far away from the healthy case with respect to $C D$ values and time for improvement. The high-load CD values of Benjamin reach the values of healthy pupils but with much more time of exercising 


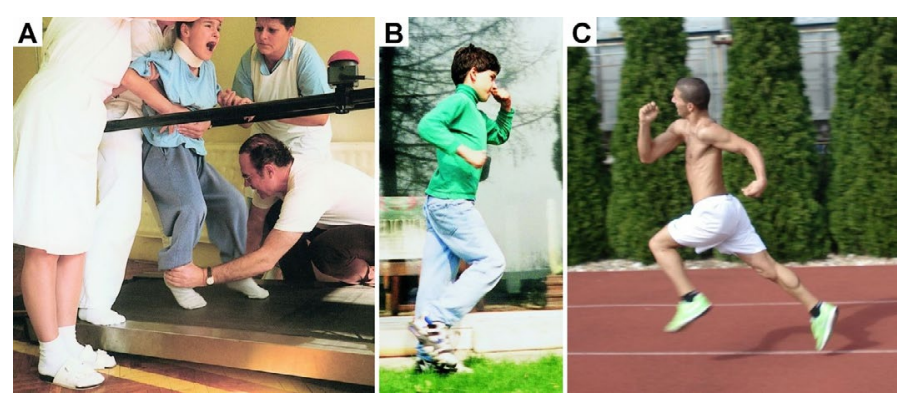

Figure 61. Improvement of locomotion over 17 years of the patient Benjamin who suffered a severe brain injury in a car accident. In the final stage of vigilant coma treadmill walking was started. The Author supported the walking pattern (A). Rather quickly he re-learned running (B) and after 17 years of CDT and sports training he became able to run $100 \mathrm{~m}$ in $14 \mathrm{~s}(\mathrm{C})$

functioning brain. Pushing buttons and working with an iPhone are no substitutes for old-learned movement patterns. The brain development of such persons is poorer and has less possibilities for repair. The same holds for continuous writing and digital writing at school.

\section{Recovery from extreme severe brain injury through CDT - Coma recovery}

Probably the most scientifically interesting success in brain repair through CDT is the achievement to bring a permanent coma patient back to life (Manolis, Figure 63) [39,60,61,68]. As will be shown, the rehabilitation centers are theoretically and practically that much out-ofdate that the relations have to take over the treatment, after the intensive care period. Further, an MRI is only of limited help. One has to treat the patient with an efficient therapy and see then what the possibilities are of the injured CNS of that patient (Figure 63).

Following an extreme severe brain injury in a car accident, a 22 -year-old male patient lost approximate $50 \%$ of the brain (Figure $64 \mathrm{~A}$ ) and several brain parts became damaged, when a metal shard went into his brain, and fall into the permanent coma state with inefficient physiotherapy. One year after the accident and conventional rehabilitation, coordination dynamics therapy (CDT) was started with 20 hours per week. Through 3 years of CDT his cardio-vascular performance improved, but he was still in the coma. After 4 years of treatment he reached the minimally consciousness state [74] and following 5 years of CDT, he had recovered fully from coma. Some movement functions re-appeared, and he became able to communicate with the surrounding. Following 5.5 years of CDT he could move better and say the word 'ma' instead of mama. It was hoped that he could relearn a few words. After 5.7 years of intensive CDT, he rather suddenly became able to speak again. Probably the neural networks for speech were operational, but they had to be connected to mind. He could precisely repeat every word in English or Greek (mother tongue), but he was not able to have a conversation. The cognitive functions were still missing. Following 6 years of CDT the patient Manolis became able to exercise a bit on a special CDT device by himself. His higher mental functions, including memory, improved and he became able to communicate now via speech. Because of the speeding up of the repair, when the patient became conscious, more brain repair seems possible through further intensive CDT. Following 6.1 years of CDT, the scratching automatism returned, and he could when asked take numbers from a tablet but could not re-place them (Figure 63C). He got nervous because of the missing fine control. The kissing was elegant, as if kissing is an automatism. It seems that the automatisms returned well, probably stored in the cerebellum which may not had been damaged, but for the execution of movement patterns, the sensory-motor cortex is needed, which was mainly lost in the right hemisphere and may be a bit damaged also in the left hemisphere.

Another 25-year-old man fall into coma following a car accident. His MRI looked nearly normal (Figure 64B). The physicians thought at the beginning that the patient would soon recover from coma and walk out of the hospital (Figure 64). For consultation, the Author saw the patient after being 8 months in the coma. The Author tried to communicate with the patient via eye blinking and hand grip power. The patient did not communicate by eye blinking and also the Author could not feel any activation of hand and finger muscles when giving the command to chase the hand. The patient was then transferred to the wheelchair and the legs were mounted to a special coordination dynamics therapy device. The Author took the hands of the patient and turned together with him the handles. During the passive exercising the patients opened the eyes strongly and the facial expression changed. He looked more alert. Suddenly after $20 \mathrm{~min}$ of exercising arm muscle contractions occurred in the flaccid arms. It seemed as if the patient blocked the movement exerted by the Author.

When patients with severe brain injury try to help during exercising, their CNS has stress and reacts with spasticity. Even though the patients want to help during exercising they block the movement unwantedly.

After exercising 40 minutes excluding a few breaks of $5 \mathrm{~min}$ the Author was checking ones more the consciousness. He took ones more the two hands of the patient and asked to chase the hand. Now the Author could feel finger muscle contractions and could see also the small fingers moving. Georgios had become able to contract hand muscles on command first time after 8 months. Then the Author asked the mother to do the same procedure. The patient Georgios contracted hand and finger muscles on command as with the Author but did not want to let the mother's hand free as if he would need her hand for safety/anxiousness reasons. Obviously, Georgios had reached the minimally conscious state at least in the short-term memory.

Next day the Author proved again the consciousness state of the patient. The important question was whether the patient had reached the minimally consciousness state permanently or only transiently. The patient did not communicate. He had lost the minimal consciousness state. But when exercising again on the special CDT device for 40 minutes, the patient could communicate again with hands and fingers as the day before.

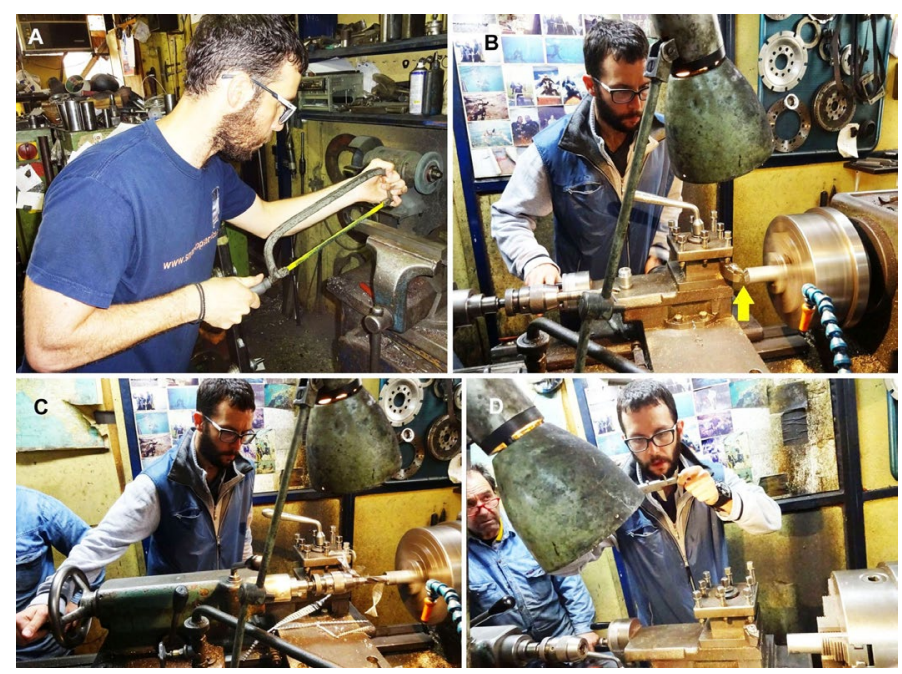

Figure 62. The patient Sotiris, 10 years after a severe brain injury, during sawing (A) lathe (B), drilling (C) and caliper (D) 

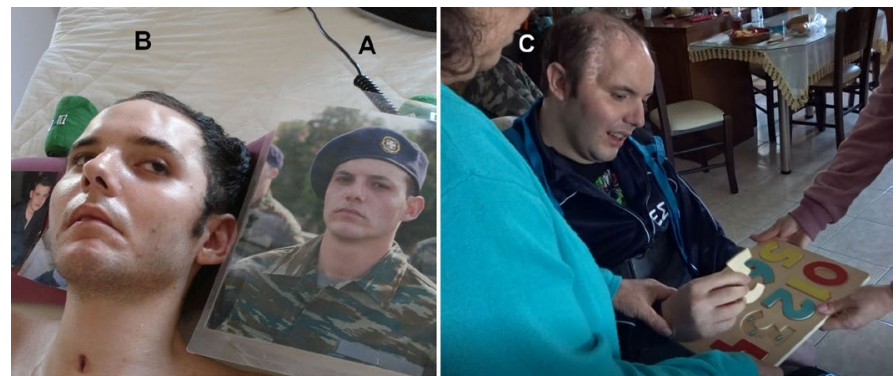

Figure 63. A,B. Picture of the 23-year-old patient Manolis being 1.5 years in the vigilan coma following a car accident (B). The impression in the face is unhealthy. The photo shows the patient 2 years before when he was in the army (A). C. Manolis following 6.1 years of CDT during taking and replacing numbers. Note the rather healthy facial expression

This training and communication with the coma patient Georgios are for several reasons important. Firstly, the coma patient Georgios could be transiently brought out of coma repeatedly, which was not possible in the 8 months of conventional therapy. With conventional physiotherapy his face expression became also livelier as the mother said, but he did not come out of coma. Efficient integrative (coordinated arms and leg movements) neural therapy seems to be needed to reach consciousness because it was at this stage of neural network organization only possible to reach consciousness when exercising on the special CDT device. Secondly, consciousness seems to be an integrative neural network state of the CNS. Thirdly, it may be that in this patient Georgios the injured 'Ascending reticular activating system' (ARAS) was activated more efficient when exercising with him on the special CDT device than by conventional physiotherapy. The coordinated movement induced afferent input made the network/pathway ARAS better working in the way that the network/pathway nerve cells reached the threshold earlier because of the more coordinated endplate potential input (Figure 49). Fourthly, with repeated exercising the minimally conscious state will be first reached only transiently in some severe brain injuries and only later the consciousness state will become permanently. Most patients with severe brain injuries stay continuously conscious once they left the coma state. But in the case of Georgios the minimally consciousness state was reached first in the short-term memory. Also, the mother of the patient Manolis reported that she could communicate with her son best after exercising on the special CDT device. Further, the mother's voice seems to be most efficient to reach the patients consciousness. Fifthly, the patient Georgios has a good prognosis to reach full consciousness and a meaningful life if CDT is administered continuously, because his lost amount of nervous tissue is very small in comparison to that of Manolis (Figure 64). Sixthly, depressing for the Author, the parents still went on with the conventional physiotherapy in the rehabilitation center and the patient is now nearly two years in the coma.

An 18-year-old woman suffered an extreme severe brain injury in a motor cycle accident. When the Author saw her, she was in coma and had no spasticity. She had a flaccid paralysis, which has a very poor prognosis, as in SCI. Even the Author thought that nothing can be done any more for her. As one brother of the mother said that two weeks earlier, she contracted the hand on command. Within two weeks the pathologic processes had progressed that much that she was fully in the coma then. The strategy of the Author is that one has to try and not to believe. With the help of the mother and the two brothers of the mother (the father died in the motor cycle accident) the young lady Georgia was transferred to the wheelchair and the legs were mounted to a special CDT device. The Author took the hands of the patient and turned the handles. The mother and her two brothers were supporting the posture. After $30 \mathrm{~min}$ the training was terminated with no success.
Next day it was tried again. This time some muscle activation could be felt. The physiotherapy of the rehabilitation center did not want to include the exercising on the special CDT device in their program. They went on with their inefficient out-of-date physiotherapy. The Author advised the relations of Georgia to perform the treatment at home. In the rehabilitation center Georgia has no prognosis.

In conclusion, the possibility that a patient with a severe brain injury may reach the consciousness state only transiently and only if efficient neuro-therapy is administered makes a prognosis of coma patients very difficult. But the issue is very important with respect to end-of-life decisions and removal of organs from brain-dead humans for transplantation.

The importance of this brain repair is not only that long-lasting coma patients can possibly be brought out-of-coma, but that this brain repair is based on theory, supported by human neurophysiology and the system theory of pattern formation.

\section{Facial expression}

Facial expressions are vital to social communication between humans (Figure 65). They are caused by the activation and movement of muscles that connect to the skin and fascia in the face. These muscles move the skin, creating lines and folds and causing the movement of facial features, such as the mouth and eyebrows. These muscles develop from the second pharyngeal arch in the embryo. Facial expressions are easily recognized by another person and is also used when performing CDT to judge repair-improvement (Figure 57). The improvement of brain repair can also be seen in the facial expression of the patient Manolis (Figure 63B, C). Especially in children one can improve their motivation to train at limits if the therapist is suggestive (facial expression and sound). In the upper right of Figure 45 the Author was very suggestive to stimulate the cerebral palsy girl Sophie to train more to see an improvement of brain functioning in the short-term memory.

Neural mechanisms in face perception: The amygdala plays an important role in facial recognition. Functional imaging studies have found that when shown pictures of faces, there is a large increase in the activity of the amygdala. The amygdala receives visual information from the thalamus via the subcortical pathways. The amygdala may also have a significant role in the recognition of fear and negative emotions. It is believed that the emotion disgust is recognized through activation of the insula and basal ganglia. The recognition of emotion may also
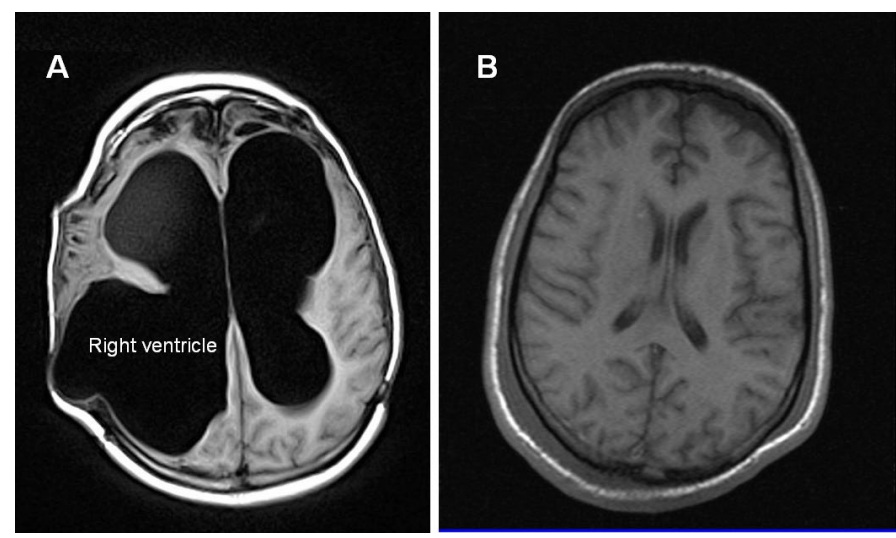

Figure 64. Lateral ventricles of the patient Manolis with a very severe brain injury and loss of brain parenchyma (A) and for comparison the ventricles of rather physiologic size (B). It is obvious that the patient Manolis has strongly enlarged ventricles 
utilize the occipitotemporal neocortex, orbitofrontal cortex and right frontoparietal cortices [69].

\section{Stroke}

The problem which the former coma patient Manolis had with the left hand and arm, after losing big parts of the right hemisphere, is similar to that of stroke patients. The famous violin player Jan Berky-Mrenica suffered a stroke and could after the stroke first only play with the left hand. His son was moving the violin bow during a concert after the stroke (Figure 66B). Through CDT Jan Berky slowly re-learned to use the right hand and arm again and could play a little bit the violin again by himself (Figure 66D) [49]. In comparison to this stroke patient, Manolis was, when playing violin (Figure 29) [61] in the stage of recovery as Jan Berky was in Figure 66C. Whether Manolis can reach the repair stage of Jan Berky is unclear but unlikely, because the injury in Manolis was more severe and was not only sited to the right hemisphere.

Jan Berky succeeded, by using CDT, to play violin again in a concert. He was nearly cured by CDT. The beginning of the therapy of Jan Berky can be seen on a video film, including his own music (see videos in the internet page www.coordination-dynamics-therapy.com). Figure 68 shows another stroke patient, who improved through CDT. Musicians have a good prognosis because they are used to play their instrument 4 to 5 hours per day and seem to have the mental discipline to perform CDT 4 hours every day. Further, the old-learned pattern, playing an instrument, is integrative stored and will help in brain repair. Athletes, on the other hand, have mostly no good prognosis, because they often seem not to be interested to train at limits for longer times if they cannot win a medal.

\section{Parkinson-blood pressure reduction}

Patients with Parkinson's disease benefit very much from CDT. After exercising the first time for one hour on the special CDT device, one can see then already substantial improvement in walking. It was shown that patients got already better with a low intensive level of therapy [57]. But is it possible to cure the disease or to keep the level, that means to not get worse with ageing? The only problem for this group of patients is the mental discipline to train close to limits. The Author asked 10 patients, who were using the special CDT device unregularly, whether they would like to increase the intensity of treatment and to be supervised by the Author. Three of the 10 patients with Parkinson's disease agreed to start the study of intensive therapy. After two months also these three patients stopped the more intensive therapy.

Luckily, one patient with Parkinson's disease contacted the Author for supervision of an intensive therapy. He exercised at least one hour on the special CDT device, including high-load exercising. Additional he was training crawling, walking, jogging, cycling and swimming. Altogether, he trained between 15 and 20 hours per week. For two years he could keep the tremor level (or reduced it a bit) and his movement performance got better. Most obviously, he learned to walk better. He had also increased blood pressure and had medication for that. CDT could still reduce his blood pressure further.

This patient with Parkinson's disease obtained angiotensin receptor blocker, thiazide-diuretic and $\beta$-Blocker for antihypertension therapy and drugs to dilute the blood and improve prostate function. If coordination dynamics therapy (CDT) could reduce the arterial blood pressure, then the medication could be reduced. CDT was started at the time when the Parkinson disease was diagnosed, four

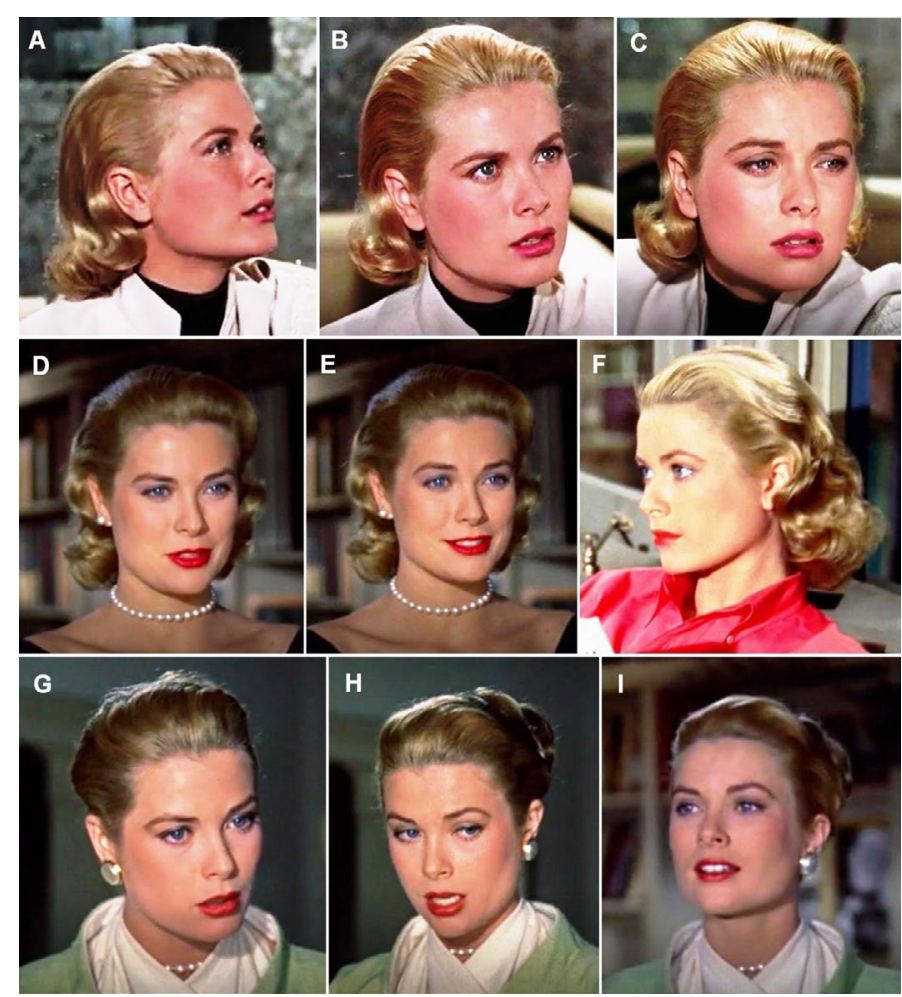

Figure 65. Facial expressions of the actress Grace Kelly from the Alfred Hitchcock's films 'To catch a thief' (first row) and 'Rear window' (second and third row). Grace Kelly was able to express different emotions in her beautiful face. Maybe the poets Goethe or Schiller would have been able to describe with words such emotions expressed by Grace Kelly, the Author for sure not. It is unbelievable how the nervous system can activate and fine control skeletal and smooth muscles to move the skin, creating lines and folds and causing the movement of facial features, such as the mouth and eyebrows. What a pity that Grace Kelly did not continue to make films with Alfred Hitchcock. With little changes in her face from D to E, Grace Kelly was able to show a different emotion. In C she was able to show sadness without tears. And in I, the charisma is great. From D to I the lips are too red, impairing the beauty of her face. But for speech therapy such strongly colored lips are helpful

years ago. The antihypertensive drug therapy was started already six years ago, that means before Parkinson's disease was diagnosed and treated.

Figure 67 shows that during exercising on the special CDT device the blood pressure lowered in the patient with Parkinson's disease, and also in the healthy case, when turning 1000 to 2000 times (Figure 67). During the 30 to $60 \mathrm{~min}$ the lowering was most pronounced, at least in the healthy case. But in this patient with Parkinson's disease and antihypertensive drug therapy the arterial blood pressure lowered less strong than in the normal case (Author). Interesting is that the pulse rate in the patient was lower than that in the normal case, which is probably due to the administered $\beta$-Blockers. Anyhow the blood pressures lowered also in the patient in spite of the plenty of drugs being administered. It seems that CDT improves the general health, including the improvement of the blood pressure, through movementbased learning.

This healthy treatment may be an alternative to antihypertension drug therapy, especially of the beta blocker administration. It seems likely that this device-guided aerobic exercise for 30 to $60 \mathrm{~min}$ can be used in Angina pectoris.

\section{Ageing}

Coordination Dynamics Therapy (CDT) cannot only improve the health of patients with CNS injury but can also improve the health in 

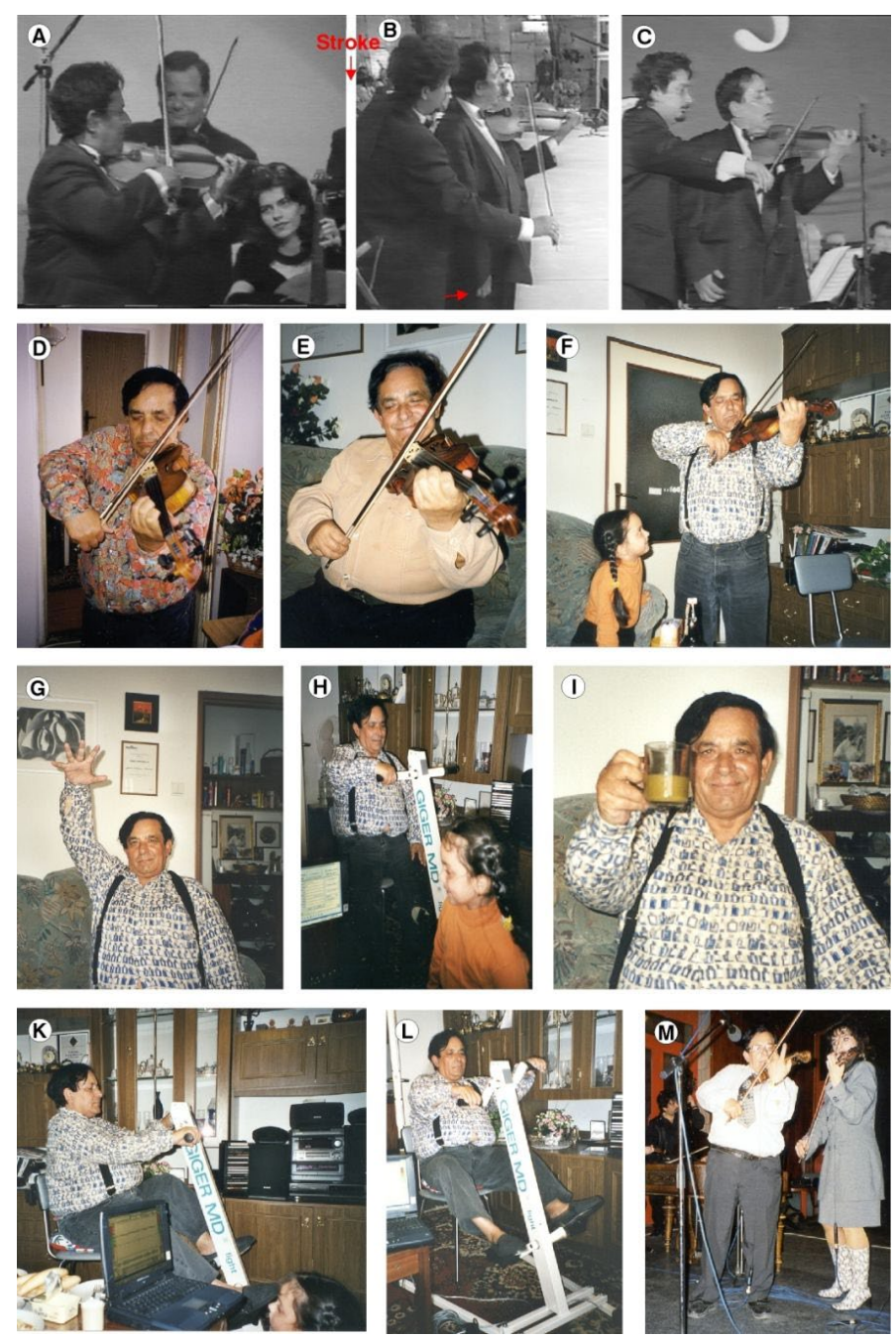

Figure 66. Coordination dynamic therapy-induced stroke recovery of a now 62-year-old musician (Jan Berky-Mrenica) made visible by the improvements of the movements. A. Before stroke; the musician is performing with his orchestra. B, C. After the stroke the patient is performing in concerts with his son; he is holding the violin with the left hand and his son is moving in coordination the bow. In B the plaggic arm is hanging down and the hand is spastic. In C (later) the now paretic arm is coming a bit upwards (is already a bit flexed), as if the paretic side wants to contribute to the coordinated movements of the old-learned CNS activation 'performing violin'. D. The patient can just hold the bow by himself 3.5 years after the stroke; the positioning of the fingers is very poor. At this stage coordination dynamic therapy was started, supervised by the Author. E. With ongoing therapy the finger positioning for holding the bow was already better. The shoulder is more downwards because the patient has already a bigger amplitude for arm movement when moving the bow. F. Five years after the stroke the movements of the left arm, hand and fingers are not far from normal again. G, H, I. The patient can fully lift the formally plaggic arm and can move the fingers $(\mathrm{G})$, can turn the device (hand and arm power came back) $(\mathrm{H})$ and can hold a cup for drinking (I). K, L. Patient exercising on the special coordination dynamic therapy device. In $\mathrm{L}$ he is training the finger positioning for holding the bow when playing violin. M. Patient playing violin again in a concert with his daughter. He is playing the first, she the second violin. The son is on the other side in the background. Note that the positioning of the right hand and fingers is poor because of the stress to organize the concert and performing for the first time after the stroke again. What cannot be seen in the picture is that the music he played was not ruined by the stress. Two days later, when the stress had subsided, the positioning of hand and fingers was physiologic again $(\mathrm{E}, \mathrm{F})$

aging to live longer with a better quality of life, may be by 20 years. The improvement of health in aging is shown for a patient with severe cerebrum and cerebellum injury, and the Author following cancer treatment. Both were fighting for a fountain of youth.

The dream of the fountain of youth: It is a many generation dream of a "Jungbrunnen" (fountain of youth) to become young and healthy again. The famous painter Lucas Cranach had pictured such a "Jungbrunnen". In his painting the old people enter the "Jungbrunnen" one side and leave it young and healthy on the other side. Such a dream cannot become reality by using CDT. However, it is possible to reduce the biological age through repair. It seems possible to live longer with a better quality of life via CDT which also holds for patients with nervous system injuries.

Since exercising on the special CDT device is not only improving the fitness, but also the functioning of the nervous system, and the nervous system is involved in nearly all body functions, including the cardiovascular performance and microcirculation, it seems possible to live longer with a higher quality of life upon performing CDT every day for one to two hours. Continence functions will also improve. Healthy nutrition, training on the CDT device, good sleep, and little stress are beneficial for healthy living. Especially eating garlic and ginger and exercising on the special CDT device seems to be beneficial to improve the blood supply of the body, since the exercising on the special CDT device is activating the arterioles, via the autonomic nervous system, which contribute substantially to the microcirculation. Also, the microcirculation of the lymph vessel system is improved. Exercising on the special CDT device is beneficial for sleeping well. Especially when waking up at night and not being able to sleep again, a 30min training will help to start to sleep again. How the mechanism of coordinated arm and leg movements can influence the sleeping pattern is unclear. Maybe the stability of the sleeping pattern is improved in the short-term memory. An old saying in medicine is "you are as old as your blood vessels are". If the functioning of the blood vessels can be improved and new nerve cells can be built upon CDT, then also eye functions should become better. Operations may be needed only later. Movements can anyway be improved in elderly. By sitting on a chair or lying during exercising, the elderly can even exercise on special CDT devices if they have prostate problems. By improving the cardio-vascular and lymph vessel performance, the risk of stroke, heart attack and cancer can be reduced. After stroke CDT can improve the health as shown in (Figure 68).

Aging in cerebellum and cerebrum injury: CDT of changing intensity was mainly administered at home for 19 years to a 58-year-old patient who had suffered a severe cerebellum injury and an injury of the frontal and parietal lobe. Motor, vegetative and higher mental functions could be improved substantially. At an age of 77 the health of the patient was still slightly improving with 10 hours CDT per week. Especially the clearness of the speech became better.

At an age of 55, the Hofrat Dr. Dieter Cwienk suffered a severe cerebellum injury and an injury of frontal and parietal lobe. The wife was advised to give him to a residual care home for elderly. Even though the patient could respond only with one finger to commands of his wife, she started to fight for her husband. The first 3 years, conventional therapy was administered to him with little progress. At an age of 58 he started CDT for 19 years now. During the first 10 years of therapy substantial progress of the patient was achieved $[3,54]$ as can also be seen from the expression changes of his face (Figure 57).

With respect to aging it is of interest to find out what is the outcome of the such patients on the long term. For patients with mild poliomyelitis it is known that with aging they cannot walk any more (post-polio syndrome) but need a wheelchair if no CDT is administered to them. In the case of Dr. Cwienk it was shown that the therapy under professional conditions was much more efficient, namely by a factor of 150 as quantified by low-load coordination dynamic values [3]. In the 2.5 years before 77 , the patient was training by himself 10 hours per 


\section{Lowering of blood pressure via Exercise Resting blood pressure [mmHg]}

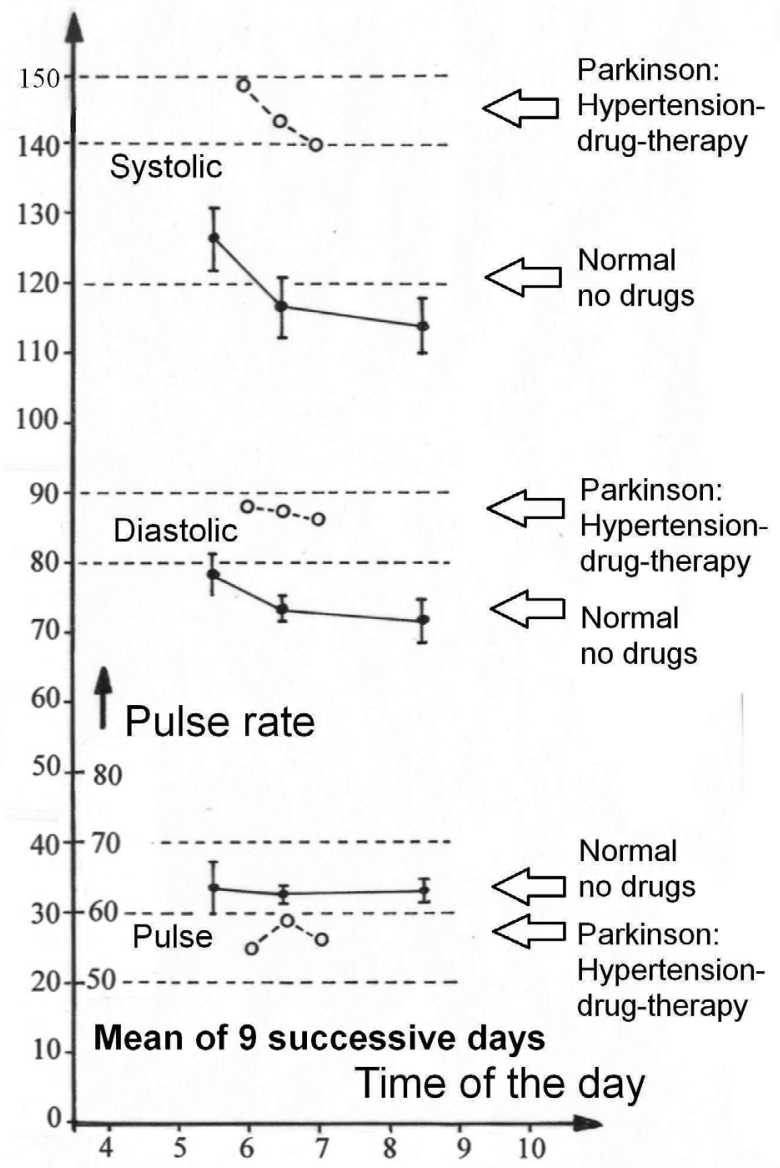

Figure 67. Lowering of the blood pressure of a patient with Parkinson's disease and hypertension. The blood pressure (open circles) was measured before exercising and after 1000 and 2000 turns. For comparison normal blood pressure lowering (solid line) of the Author is shown, who takes no drugs. Note that the lowering in the normal case is stronger. The values are means of 9 or 10 successive days. For the normal case (Author) standard deviations are indicated

week on the special CDT device and the treadmill. With this amount of exercising he could keep the level and could even get still a tiny bit better.

Figure 69 shows the patient during measuring his coordination dynamics in his office and therapy place (Figure 69).

As the low-load and high-load coordination dynamics values showed, the organization of his nervous system from the aspect of the coordination dynamics values did not get worse with aging.

Even hand and finger functions improved a bit in the patient Dr. Cwienk, the handwriting did not. The patient has to train more the fine control of fingers, hands and arms in everyday life to improve it. Because of some similarity between repair and development, this finding indicates that during development the cursive hand writing should not be given up in schools.

Even with 78 (2018) the patient with the severe cerebellum and cerebrum injury improved his health when performing sufficiently CDT. When he stopped transiently CDT or reduced its intensity, his health got worse.
Competition between a good functioning nervous system and muscle power: High-load coordination dynamics comparison between the severely brain-injured patients Benjamin and Sotiris and the fit ageing Author: The health in ageing through CDT is demonstrated by the comparison of the high-load CD values of the Author with the ones from the brain injury recovered patients Benjamin (29 years old in 2018, Figure 61) and Sotiris (32 years old in 2018, Figures 28, 62).

The patient Benjamin could reach after 17 years of CDT in 2017 a value of 50 when exercising 30 hours per week (Figure 70). But when he had a lot of stress and may be went over the limit of exercising, his physical performance got worse. His high-load value was over 60 in 2018 (Figure 70). His coach, training him to run faster (faster than $14 \mathrm{~s}$ per $100 \mathrm{~m}$ ) and jump longer distances, did not understand the functioning of the special CDT device and put some pressure on him not to use the special CDT device. Probably, when Benjamin could reduce the stress and would not overload himself any more, his highload CD values would go under 50 again, which is indicated in Figure 70 by a dashed line.

The patient Sotiris could improve his CD values over the years with 30 hours CDT per week (Figure 30). But when he studied and could train only 7 to 10 hours per week, he got worse again and had a high-load CD value of over 100 (Figure 70). When he finished successfully his university study, got rid of the study stress and could exercise again 30 hours per week, he got much better. His CD values went continuously under 50 with some disturbance when upgrading the therapy. He even became able to beat the Author at the end of 2018 (Figure 70 ). When Sotiris reached the value of 40 , he became able to speak more clearly. Therefore, not only his CD values got better with the intensive therapy but also the clinical performance improved (the speech).

The Author reached in 2016 with approximately 15 hours CDT per week the dream value of 30 (Figures 70). After that, the CD value went into the direction of 50 in 2018, which means, he could by far not hold the dream value. An explanation would be that he got older (depressive for the Author) and lost power which is also needed for a good high-load CD value besides a very good coordination between arm and leg movements. Other reasons like illnesses (infections) may also have ruined the dream high-load CD value of 30 . With intensified CDT and including exercising at $200 \mathrm{~N}$, the Author could reach a value of under 40 again. But this good health value is still far away from the dream value of 30 . But the benefit from this hard work was that a small wound in the face, which did not heal for two years, healed. It seemed
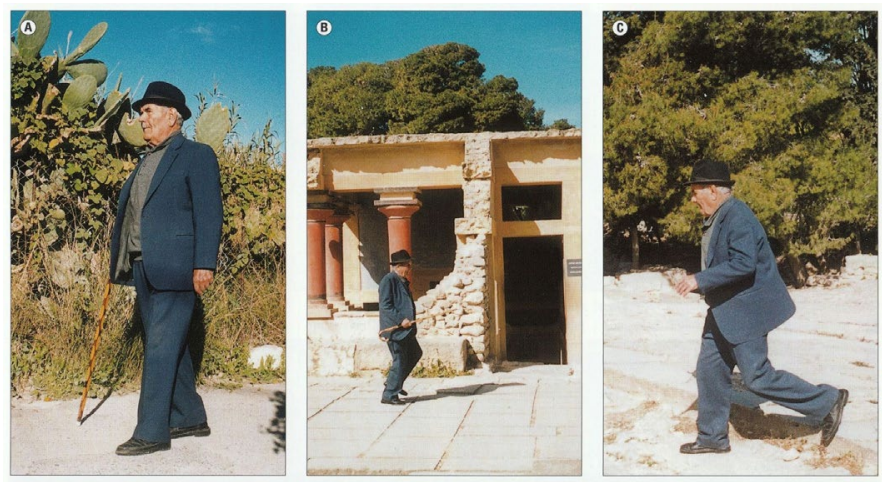

Figure 68. 82-year old patient after severe stroke. A. Patient is using the stick during walking. B,C. Via CDT the patient can run again over Minoan culture (Knossos, Greece) 


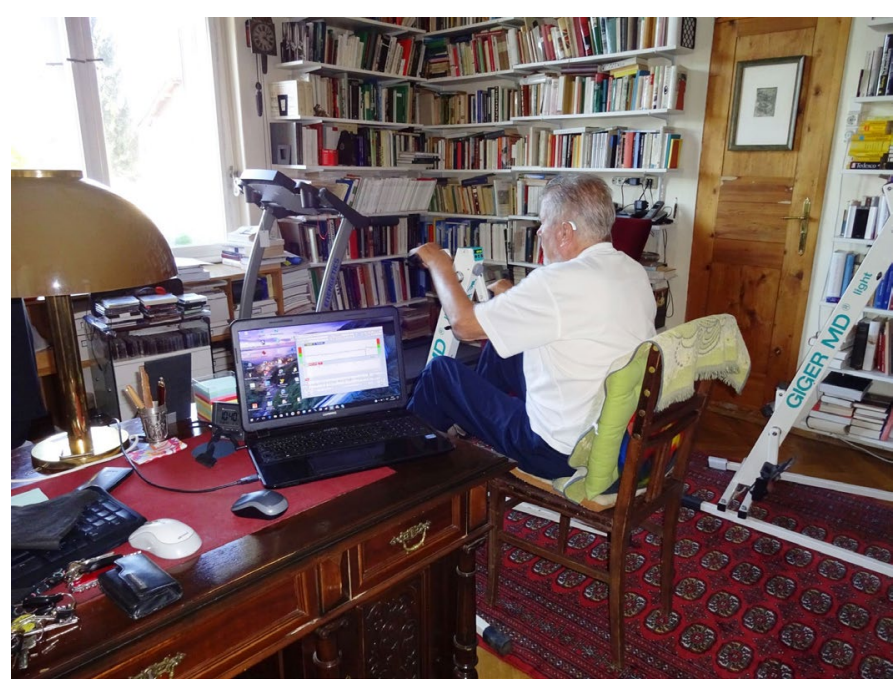

Figure 69. 77-year-old patient, 22 years after a severe brain injury (cerebellum loss approximately $60 \%$, injury of the frontal lobe and the parietal lobe) during exercising on a special CDT device for measuring the low-load and high-load coordination dynamics in his office, which is also his therapy place. His own larger training device for exercising in the sitting position is on the right side. His treadmill is in front of him

that also the eye function improved a bit, which was impaired following chemo and radiation therapy during cancer treatment 10 years ago. Even though it was very hard to exercise at $200 \mathrm{~N}$, the Author got the benefit of health improvement. With respect to health improvement it is interesting and very important that during exercising on the special CDT device, especially at higher loads, the repair system finds the places in the body where something has to be repaired. Often these places are itching.

This high-load CD values show only a part of the quality of CNS functioning, but objectively. Benjamin, for example, runs very much faster (Figure 61C) than the Author. The competition, pictured in Figure 70, helps Benjamin, Sotiris and the Author to fight for a better health. Further, when the Author trains similar movements as the patients, he can feel what he expects from his patients.

\section{Cancer growth inhibition}

Authors own experience with the anticancer effect and general health improvement through CDT: The Author suffered a 'squamous cell carcinoma (epithelioma)' (a malign tumor) in the maxilla (stage between 1 and 2). The tumor was removed by surgery and a neck dissection performed: Two lymph nodes with formation of metastases were removed and two stages of further lymph nodes and lymph vessels were removed for safety reasons. Radiation therapy and chemotherapy were administered to the tumor area to reduce the risk of tumor recurrence from $30 \%$ to $15 \%$. To mitigate the side effects of the anti-cancer treatment, the Author performed CDT for fifteen hours per week. Three biopsies of strange growing in the mouth in the following five years and other diagnostics, including two PETS, showed no sign of malign growth. The exercising on the special CDT device involved coordinated arm, leg and trunk movements and additionally simultaneously coordinated neck, tongue and lip movements. The training improved body health in general and improved impaired brain health because of radiation and chemo therapy and reduced chronic stress because of anxiety of a recurrence of the cancer. As the Author found out later, the exercise also inhibited cancer growth (exercise oncology).
One year after the tumor extirpation and neck dissection, a reconstruction of the maxilla was performed. The Author was fit by the time he went for the fibula-transplantation operation and the hematocrit was high (no blood needed). He even exercised one hour before the operation on the special CDT device to counteract the down-regulation of BDNF and other factors due to the coming stress event (operation). During the nine-hour operation, pieces of the fibula and the skin with its supplying artery and vein were removed from the right leg. Bone and skin were placed to the former site of the tumor and artery and vein were connected with the temporalis blood vessels. The transplant had to receive blood supply within seven hours in order to avoid rejection. The Author was informed that during the long-lasting operation he will get edema and breathing difficulties because of the removed lymph nodes and vessels following the previous neck dissection previously. However, only minor neck edema occurred during the operation because the CDT had partly repaired the lymph system through the performance of CDT. One day after the reconstructive operation, parts of the transplant were blue, indicating poor blood supply, even though the transplanted artery was open, and blood was supplied. The surgeon informed the Author that he would lose some tissue because of poor blood supply.

The Author, having brought a special CDT device into the hospital, exercised on the special device, in spite of the many tubes he was connected to, to improve the blood supply to the head including the transplant site. As the result of the enhancement of blood circulation, the transplant area received more blood and healed fully. No transplanted tissue was lost. The surgeon was amazed that his 9-hour lasting complicated work was fully successful. He himself said that the reconstruction was $100 \%$ successful. Therefore, CDT had helped to reduce the risk of severe neck edema occurring during the operation and a tracheotomy could be avoided and CDT had helped to improve

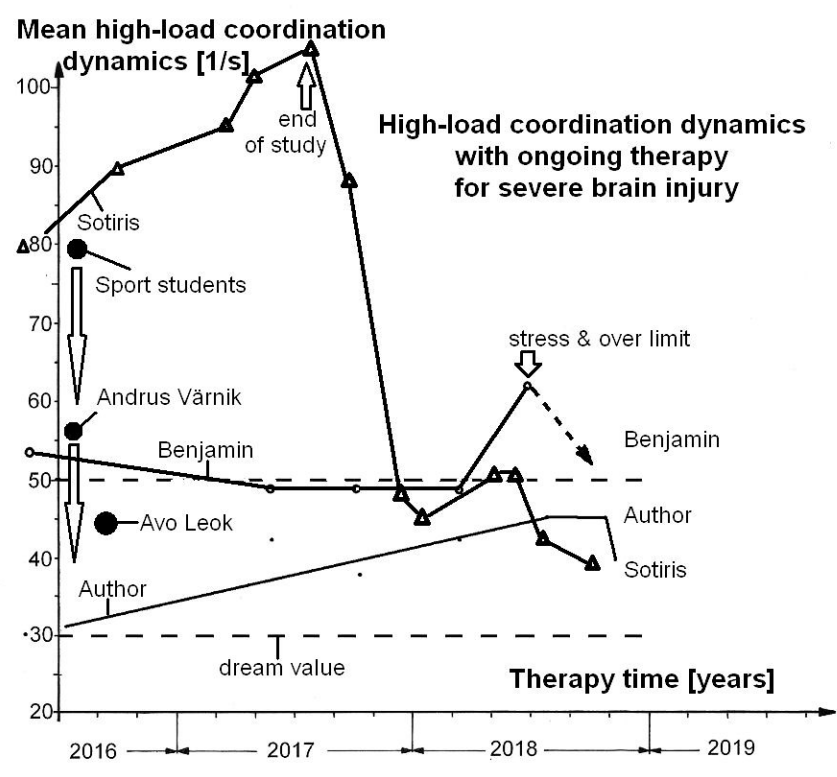

Figure 70. Mean high-load CD value comparison with ongoing CDT of the brain-injured patients Benjamin and Sotiris and the healthy aging Author. The mean of 2 to 6 values were averaged for a single value and the mean of forward and backward exercising was taken. The high-load values of Andrus Värnik and Avo Leok are included, measured in 2002 and 2007 respectively; the arrow indicates Värnik's probable improvement with further CDT. The values of sport students and the value they can reach (arrow) with a few times exercising. The approximate values of physiotherapist (130), gymnasts (150) and musicians (150) for exercising the first time are not fitting into the figure. Note that the values of Benjamin, Sotiris and the Author are in the range what top athletes may reach when they exercise at least a few times on the special CDT device 
the blood supply also in the head so that the transplant was fully accepted. The Author's hour training before the operation may have helped in this long-lasting and difficult operation and may have helped to improve the lymph circulation in the short-term memory to reduce edema during the operation.

In conclusion, all surgery went well, aided by the continuously performed CDT. CDT had helped to improve the general health after cancer treatment and may have helped to avoid cancer recurrence. CDT included not only the exercising on the special device but also fast walking and jogging. Since the Author had a hip necrosis on the right side and could only walk with pain, he decided to get an artificial hip to be able to walk well again and to be able to jog. The hip operation was successful, and a special CDT device was used post-operatively, so that the Author could again perform the whole movement program of CDT.

Important to note is that following the neck dissection (and tumor resection, radiation and chemo therapy) the lost or impaired automatisms such as taste, swallowing, coughing and others, partly reappearing spontaneously, recovered better due to CDT. The Author believes that the exercising of the complicated coordination's between pace and trot gait, when turning on the special CDT device, which stimulate the autonomic nervous system, helped to achieve better repair. Even though the eyes are a part of the nervous system and should be reached by CDT, the improvement of the eye functions (wetting of the cornea, opacity of the vitreous body) seems to be most difficult to repair by CDT. Ten years after the cancer treatment the wetting of the cornea and the opacity of the vitreous body had improved but were still not normal. Based upon CDT most likely new cells of the eyes were built to improve eye functions. The further repair of the mouth, throat and neck functions and automatisms improved quality of life dramatically. Even though, with 10 years of CDT not all side effects of chemo and radiation therapy had disappeared. Sleep is still more disturbed than before the occurrence of the cancer. The chemotherapy probably impaired nervous system functioning and in consequence many body regulations. It is known that the drug Vinblastine, which was administered to the Author, is nervous system toxic. The patients may get polyneuropathy.

An important question remains, can CDT as an efficient exercise partly replace chemo and radiation therapy or reduce its doses, because their side effects are immense? Nefeli, of the above case report, did not obtain chemo and radiation therapy because of the spinal cord injury. Only CDT was administered to her and so far, there is no cancer recurrence (7-year survivor). But her cancer (neuroblastoma) was not that malignant than the one of the Author (epithelioma). Exercise stimulates tumor suppressor genes, the immune system and cardiovascular performance. Prolonged fasting improves the immune system by a recycling of old or damaged immune cells and building of new healthy immune cells. A combination of exercise and prolonged fasting may be more efficient to inhibit tumor growth than exercise alone.

All the cancer patients who performed CDT after the cancer operations, including breast cancer and colon cancer, had no cancer recurrence so far [48].

In conclusion, $\mathrm{CDT}$ cannot only improve brain health in patients with CNS injury and Parkinson's disease, but it can fight against several diseases at the same time. CDT improves health in general.

CDT probably inhibits prostate cancer: Four friends of the Author at an age of between 60 and 80 got prostate hyperplasia or cancer. One friend with a diagnosed hyperplasia exercised 1000 to 2000 times per day on the special CDT device and the prostate did not get further worse during the five years. Another friend got a hyperplasia (before the development of CDT) and died after 5 years, when the hyperplasia turned into cancer. The hormone therapy was inefficient to stop cancer growth. To another friend, a permanent brachytherapy was administered, but he refused so far to exercise additionally on the special CDT device, which would be beneficial. Nothing can be done if the patient does not want to exercise and to reduce body weight. A fours friend got prostate cancer at an early stage. When the Author pushed him to exercise on the special CDT device, he argued that he was working already manually very hard (fisher man). It is inefficient to just do physical exercise; cancer growth inhibition depends on what exercise you do perform regularly.

Brachytherapy is a form of radiotherapy where a sealed radiation source is placed inside or next to the area requiring treatment. Brachytherapy is commonly used as an effective treatment for cervical, prostate, breast, and skin cancer and can also be used to treat tumors in many other body sites [70]. The treatment results of prostate brachytherapy can most likely be improved if CDT is used simultaneously.

Teaching electrophysiology: Since electrophysiology is a lost discipline, but is needed for understanding the human nervous system, it has to be taught at universities or medical schools again.

In a physiology practical during the first two years of a medical study, one could demonstrate the recording of single-nerve fiber action potentials in the frog. Since the nerves of frogs have no epineurium, extracellular single-nerve fiber action potentials can be easily recorded. Additional oxygen supply is not needed, the oxygen diffusion from the air is sufficient to keep the nervous system alive.

Figure 71 (upper part) shows the recording from a frog, which is similar to that of a human (Figure 71 lower part). Action potential amplitude (A) and duration (T) can be measured with the conduction time (ct) and the electrode pair distance (may be $10 \mathrm{~mm}$ ) the conduction velocity $(\mathrm{cv})$ calculated $(\mathrm{cv}=\mathrm{ct} / 10 \mathrm{~mm})$ (Figure 71$)$. One could then plot conduction velocity distribution histograms (Figure 71C) and look for nerve fiber groups in similarity to Figure 10 or plot the action potential amplitude in relation to the conduction velocity (Figure 71D). If one uses a skin nerve, one may get skin afferent input patterns (Figure 71B) similar to those ones in Figure 9. Such measurements would give the students a feeling and understanding of nerve fiber conduction. One could even try to build up a classification scheme for frog nerve fibers in similarity to Figure 7 and beat with quality the conventional classification schemes [71]. If everything is so easy in the frog, why then all this effort to measure in human? First, in medicine we need exact values, because the health of patients is behind, and last not least the frog is phylogenetically far away from human. The continence/ defecation is for example completely different in the frog. Frogs have an urachus and not a urinary bladder and a colon/rectum.

Even though the single-nerve fiber action potentials of the frog (Figure 71, upper part) and human (Figure 71, lower part) look similar and even the conduction velocities are similar, the membrane properties are different and also the measuring temperatures are different. The time calibration in the frog is $2 \mathrm{~ms}$ and in human $1 \mathrm{~ms}$; they are also not very different. But the measuring temperature in the frog was around $18^{\circ} \mathrm{C}$ and in human around $35^{\circ} \mathrm{C}$. To bring up the temperature of the frog to $37^{\circ} \mathrm{C}$ is impossible, because the frog dies at around $30^{\circ} \mathrm{C}$, even if they are brought from Brasilia. Probably they cool their body temperature down by evaporation. The conduction velocity depends strongly on the temperature. Therefore, a calibration relation of conduction velocities was introduced in Figure 10. 
Surface electromyography is also instructive for students, when they can see their own muscle activation in similarity to Figure 16. But single-motor unit action potentials can only made visible (Figures 17, 18) when recording from suitable patients. The action potentials from motor units are much longer and have a much higher amplitude than those of nerve fibers. A thick single-nerve fiber action potential has an amplitude of about $50 \mu \mathrm{V}$ and a duration of $2 \mathrm{~ms}$ and a single-motor unit has an amplitude of around $100 \mu \mathrm{V}$ (up to $1 \mathrm{mV}$ ), depending on the recording condition) and a duration of $20 \mathrm{~ms}$ (Figure 17).

\section{Conclusion}

Summary: Based on human anatomy, human neurophysiology, the system theory of pattern formation and genetics a new movement-based learning therapy was developed, called Coordination Dynamics Therapy (CDT), with which it is possible so far to improve CNS functioning after stroke, traumatic brain injury, spinal cord injury, cerebellar injury, cerebral palsy, hypoxic brain injury, in Parkinson's disease, spina bifida (myelomeningocele) and scoliosis. Cardiovascular performance (heart attack) could be improved, cancer growth inhibited, urinary bladder functions (continence) repaired and the immune system improved. A permanent coma patient could be brought back to life. Since CDT works to a certain extent simultaneously on all the diseases and can be applied to all ages from the baby (Figure 1C) to ageing humans, it improves health in general. To live longer with a better quality of life is possible already now through CDT.

An important aspect of CDT is the learning transfer from movements to other body functions which cannot be trained or are difficult to train. By performing movements, urinary bladder functions, speech and higher mental functions can be improved. The drawing back of CDT is that a lot of mental discipline is needed for patients, therapists and parents.

When CDT was started from the scratch with the microanatomy of nerve roots, the goal was to repair spinal cord injuries. The goal was
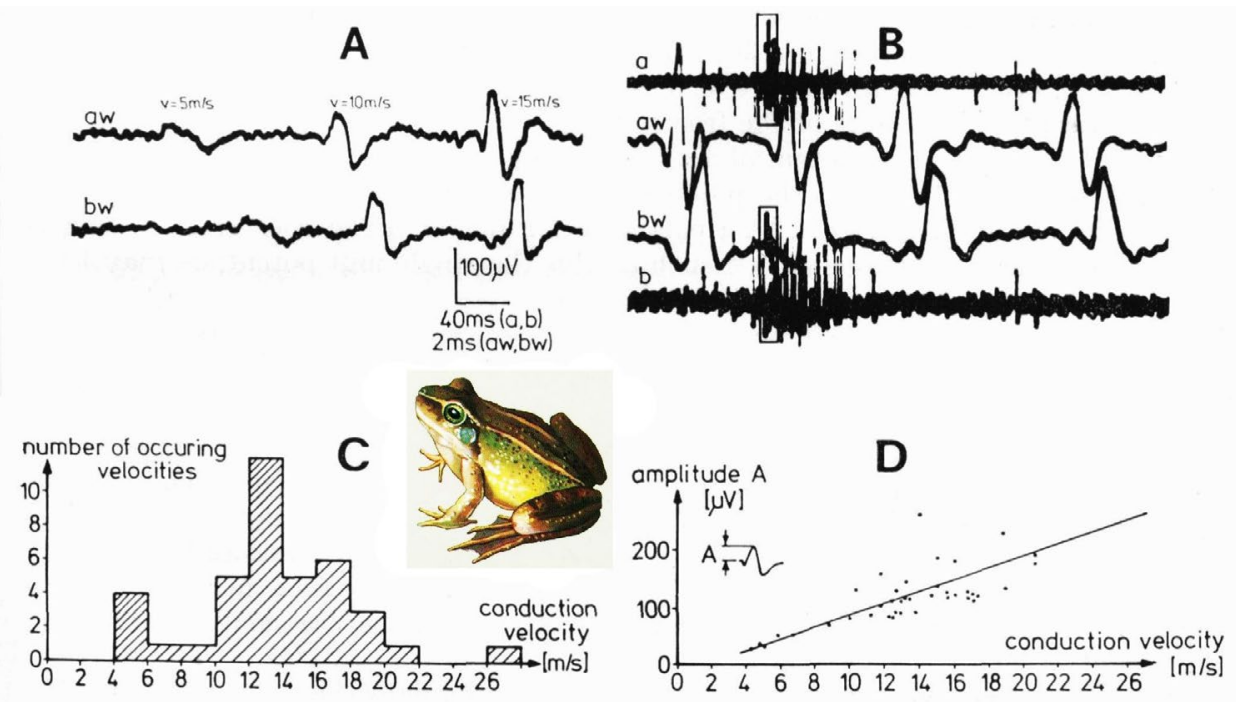

\section{Amplitude, duration, conduction time and conduction velocity of afferent single-nerve fibre action potentials}

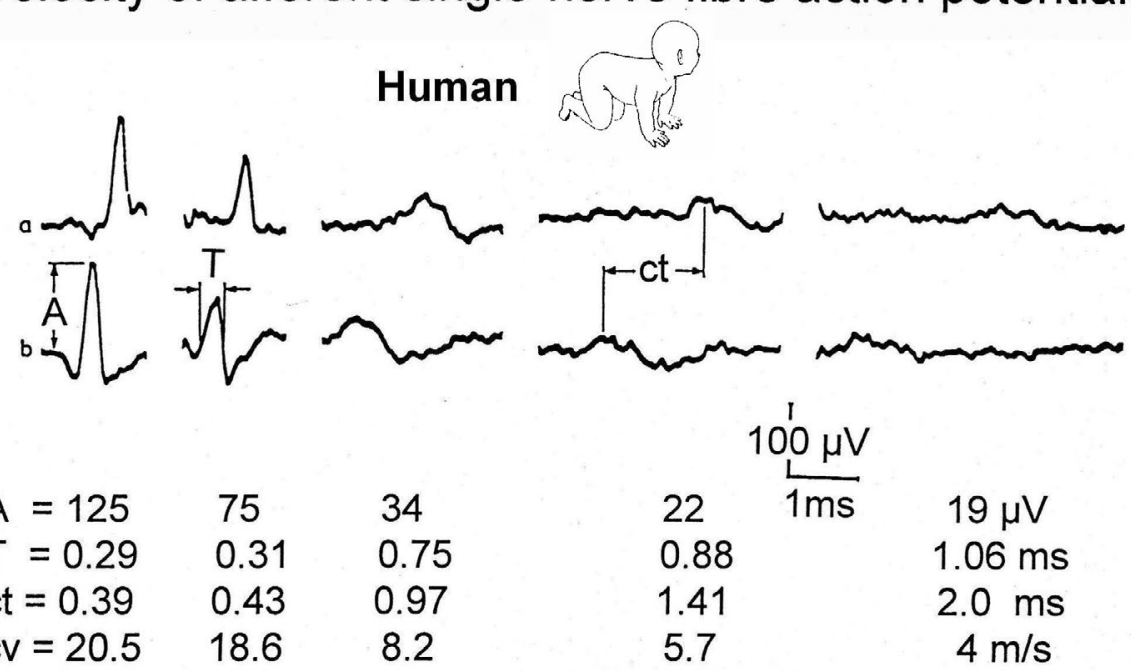

Figure 71. Extracellular single-nerve fiber action potentials in frog (Upper part) and human (lower part). The polarizations of the electrode pairs are different in the frog and human recordings 
then extended to all CNS injuries, malformations and degenerations. Now even the goal of general health improvement is achieved. CDT is the only therapy which has only positive side effects. The essential step forward in health improvement was achieved through the application of coordinated movements, which rest on the organization principle "phase and frequency coordination" of human neural network organization. These phase and frequency coordination dynamics cannot only be used for repair and health improvement but can also be used to analyze brain functioning. To copy the human brain by artificial neural networks is only possible if one understands human neural network organization also at the neuron level.

Consequences for the future: Since learning is slowly, especially with increasing age, the efficiency of movement-based learning has to be further increased. May be a combination of CDT and stem cell therapy could repair of the CNS more efficiently. Stem cell therapy administered alone was not successful, most likely because the proliferating stem/progenitor cells were not integrated into the existing neural networks. But may be CDT can improve/lead the migration, proliferation and integration of stem and progenitor cells by activating the microenvironment at the injury site to generate a repair milieu. Excitation-neurogenesis coupling is influenced by local activity, access to local activity and ability of the local environment [77]. Anyhow, CDT can improve CNS functioning and general health. Its efficiency has to be further enhanced.

With the success to repair the human CNS and the improvement of health in general, there are important consequences for the society to be done. First, human neurophysiology has also to be red at medical schools or universities, including practical's, to give physiotherapist and medical students the possibility to learn how to repair the human brain, because CNS repair is different in animals and humans. In animals mainly, the nerve fiber growing strategy is used, whereas in human's the learning is used and only $0.3 \%$ of the animal data have consequences for human patients. Second, at least in some cases it is possible to get permanent coma patients out of coma via CDT, which would otherwise stay permanently in coma or die. Rehabilitation has even problems to diagnose the minimally consciousness state [74]. Not to offer such coma patients efficient neuro-therapy means contributing to modern euthanasia.

History of the research project and funding problem: The Author is a private researcher in human neurophysiology and clinical research to repair the human CNS and is living and performing research on 1200 Euro. For the upper research project, it was impossible for him to get funding (including Christopher Reeve Foundation, Swiss National Fond and Deutsche Forschungsgemeinschaft) in the last 30 years, even though the Author studied electronics, theoretical physics $(\mathrm{PhD})$ and medicine (MD) and was post doc with Sir Bernard Katz, Huxley and Ricardo Miledi, Department of Biophysics, University College London. One was supposed to be a respected researcher, if one was able to publish at that institution and the Author was able $[75,76]$. Also, Sakmann and Neher obtained post doc education there, but they did not switch to human neurophysiology and basic medical research. Obviously, to save life of patients or to improve their health is so far of no interest for the world society if a new qualified treatment has to be used. With respect to human neurophysiology (especially electrophysiology) and clinical (human) research the universities are world-wide 30 years out-ofdate and there seems to be no interest to catch up. At the international conference IPBIS2018 in Belfast 2018 "Pediatric acquired brain injury" there was no interest for repairing the brain of children. A physician, who treated Nefeli and Sophie (Figures 1-3,15,48,55) in a rehabilitation center in Switzerland, even did not want to see the progress of his former patients through CDT!

The Author, formally from West-Berlin and not involved in politics, was performing research in East-Germany (GDR) at the Ernst-MoritzArndt-University in Greifswald. For the development of the singlenerve fiber action potential recording method (Figure 6) he was supposed to get a professorship in Dresden and for the human research to repair the human brain a special laboratory should be built [81] With the fall of the Berlin wall, this research project was destroyed, and the Author became mainly a private researcher, apart from being for 6 years the head of a research department of a well-known rehabilitation center in Switzerland.

\section{References}

1. Hodgkin AL, Huxley AF (1952) A quantitative description of membrane curren and its application to conduction and excitation in nerve. $J$ Physiol 117: 500-544. [Crossref]

2. Sakmann B, Neher E (1983) Single-channel recording. Plenum Press.

3. Schalow G (2013) Human neurophysiology: development and repair of the human central nervous system. nova science publishers, inc, hauppauge ny, usa.

4. Schalow G (2015) Repair of the human brain and spinal cord. nova science publishers, inc, hauppauge ny, usa.

5. Schalow G (2015) Neural network learning in humans. Nova Science Publishers, Inc, Hauppauge NY, USA.

6. Schalow G (1985) The problem of cauda equina nerve root identification. Zentralbl Neurochir 46: 322-330. [Crossref]

7. Schalow G, Aho A, Lang G (1987) Nerve fibre counts for an intercostal nerve to cauda equina nerve root anastomosis. Zentralbl Chir 112: 457-461. [Crossref]

8. Schalow G, Lang G (1987) Recording of single unit potentials in human spinal nerve roots: a new diagnostic tool. Acta Neurochir (Wien) 86: 25-29. [Crossref]

9. Schalow G (1987) Single unit potential amplitude in relation to the conduction velocity in frog and human. Zentralbl Neurochir 48: 109-113. [Crossref]

10. Lang G, Schalow G (1988) Experiences with the intraoperative single unit potential recording of spinal cord and cauda equina disorders. Advances in Neurosurgery 16 98-99.

11. Schalow G Barth H (1992) Group conduction velocities and nerve fibre diameters of? and ?-motoneurons from lower sacral nerve roots of the dog and humans. Gen. Physiol. Biophys 11: 85-99.

12. Schalow, G (1992) Recruitment within the groups of ?1, ?2 and ?3-motoneurons in dogs and humans following bladder and anal catheter pulling. Gen. Physiol. Biophys 11: 101-121.

13. Schalow G (1992) Ventral root afferent and dorsal root efferent fibres in dog and human lower sacral nerve roots. Gen Physiol Biophys 11: 123-131. [Crossref]

14. Schalow G, Zäch GA, Warzock R (1995) Classification of human peripheral nerve fibre groups by conduction velocity and nerve fibre diameter is preserved following spinal cord lesion. J. Auton. Nerv. Syst 52: 125-150.

15. Schalow G (2005) Tapering of human nerve fibres. Gen Physiol Biophys 24: 427-448. [Crossref]

16. Schalow G (1991) Conduction velocities and nerve fibre diameters of touch, pain, urinary bladder and anal canal afferents and ? and ?-motoneurons in human dorsal sacral roots. Electromyogr. Clin. Neurophysiol 31: 265-296.

17. Schalow G (1992) Impulse pattern, innervation densities and two-point discrimination of skin and mucosal afferents in humans. Consideration for a sensory reinnervation of urinary bladder and anal canal in spinal cord lesions. IV (IV). Electromyogr. Clin. Neurophysiol 32: 259-285.

18. Schalow, G. (1991) Oscillatory firing of single human sphincteric ?2 and ?3-motoneurons reflexly activated for the continence of urinary bladder and rectum restoration of urinary bladder function in paraplegia. Electromyogr. Clin. Neurophysiol 31: 323-355. 
19. Schalow G (1993) Spinal oscillators in man under normal and pathologic conditions. Electromyogr. Clin. Neurophysiol 33: 409-426.

20. Espinosa-Medina I, Saha O, Boismoreau F, Chettouh Z, Rossi F, et al. (2016) The sacral autonomic outflow is sympathetic. Science 354: 893-897. [Crossref]

21. Schalow G (2005) Phase and frequency coordination between neuron firing as an integrative mechanism of human CNS self-organization. Electromyogr Clin Neurophysiol 45: 369-383.

22. Schalow G (1993) Phase correlated adequate afferent action potentials as a drive of human spinal oscillators. Electromyogr. Clin. Neurophysiol 33: 465-476.

23. Schalow G (1993) Action potential patterns of intrafusal ? and parasympathetic motoneurons, secondary muscle spindle afferents and an oscillatory firing ?2-motoneuron, and the phase relations among them in humans. Electromyogr. Clin. Neurophysiol 33: 477-503.

24. Schalow G (2009) Relative coordination between neuron firing and generation of a motor program in the human cns. in: berkovsky, t.c. (ed.), handbook of spinal cord injuries, Chapter 5. 265-295, Nova Science Publishers.

25. Schalow G (2009) Impaired coordination between oscillatory firing FF and FR-type motor units in Parkinson's disease and patients with spinal cord injury. In: Berkovsky, T.C. (Ed.), Handbook of Spinal Cord Injuries, Chapter 15. 501-517, Nova Science Publishers.

26. Schalow G (2005) Tremor in Parkinson's disease patients can be induced by uncontrolled activation and uninhibited synchronization of a2-motoneuron firing to which a1-motoneuron firing synchronizes. Electromyogr. Clin. Neurophysiol 45: $393-$ 406 .

27. Schöner G, Zanone PG, Kelso, J.A.S. (1992) Learning as change of coordination dynamics: Theory and experiment. Journal of Motor Behavior 24: 29-48.

28. Zanone PG, Kelso JAS (1997) Coordination dynamics of learning and transfer: Collective and component levels. Journal of Experimental Psychology: Human Perception and Performance 23: 1454-1480.

29. Schalow G (2010) Scientific basis for learning transfer from movements to urinary bladder functions for bladder repair in patients with spinal cord injury. Electromyogr Clin Neurophysiol 50: 339-395.

30. Kelso JAS (1995) Dynamic Patterns. The self-organization of brain and behavior. MIT Press, Cambridge.

31. Haken H, Kelso JA, Bunz H (1985) A theoretical model of phase transitions in human hand movements. Biological Cybernetics 39: 139-156.

32. Schalow G, Pääsuke M, Jaigma P (2005) Integrative re-organization mechanism for reducing tremor in Parkinson's disease patients. Electromyogr. Clin. Neurophysiol 45: 407-415.

33. Schalow G, Bersch U, Göcking K, Zäch GA (1995) Detrusor-sphincteric dyssynergia in paraplegia compared with the synergia in a brain-dead human by using the single-fibre action potential recording method. J. Auton. Nerv. Syst 52: 151-180.

34. Schalow G, Bersch U, Michel D, Koch HG (1995) Detrusor-sphincteric dyssynergia in humans with spinal cord lesions may be caused by a loss of stable phase relations between and within oscillatory firing neuronal networks of the sacral micturition centre. J. Auton. Nerv. Syst 52: 181-202.

35. Passatore GM., Fillipi GM, Grassi G (1985) Cervical sympathetic nerve stimulation can induce an intrafusal muscle fibre contraction in the rabbit. In: The Muscle Spindle (I.A. Boyd and M.H. Gladden, Eds.) 221-226 Stockton Press.

36. De Groat WC (1975) Nervous control of the urinary bladder of the cat. Brain Res 87: 201-211. [Crossref]

37. Thelen E, Smith LB (1994) A dynamic approach to the development of cognition and action. MIT Press, Cambridge.

38. Schalow G (2010) Cure of urinary bladder functions in severe (95\%) motoric complete cervical spinal cord injury in human. Electromyogr Clin Neurophysiol 50: 155-179.

39. Schalow G (2017) Coma recovery achieved after 4 years of coordination dynamics therapy of a patient who lost $50 \%$ of his brain tissue in a car accident. In: "Horizons in neuroscience research. Volume 32". Editors: Andreas Costa and Eugenio Villalba. Nova Science Publishers.

40. Brown JC, Winters-Stone K, Lee A, Schmitz KH (2012) Cancer, physical activity, and exercise. Compr Physiol 2: 2775-2809. [Crossref]

41. Christensen JF, Jones LW, Andersen JL, Daugaard G, Rorth M, et al. (2014) Muscle dysfunction in cancer patients. Ann Oncol 25: 947-958. [Crossref]
42. Friedenreich CM, Cust AE (2008) Physical activity and breast cancer risk: impact of timing, type and dose of activity and population subgroup effects. Br J Sports Med 42 : 636-647. [Crossref]

43. Thune I, Furberg AS (2001) Physical activity and cancer risk: dose-response and cancer, all sites and site-specific. Med Sci Sports Exerc 33: 530-550. [Crossref]

44. Grivennikov SI, Greten FR, Karin M (2010) Immunity, inflammation, and cancer. Cell 140: 883-899. [Crossref]

45. McTiernan A (2008) Mechanisms linking physical activity with cancer. Nat Rev Cancer 8: 205-211. [Crossref]

46. Neilson HK, Friedenreich CM, Brockton NT, Millican RC (2009) Physical activity and postmenopausal breast cancer: proposed biologic mechanisms and areas for future research. Cancer Epidemiol. Biomarkers Prev. 18: 11-27.

47. Pedersen L (2016) Voluntary running suppresses tumor growth through epinephrineand IL-6-dependent NK cell mobilization and redistribution. Cell Metabolism 23: 554 562

48. Schalow G (2017) Breast cancer growth inhibition via Coordination Dynamics Therapy. In: "Horizons in Cancer Research. Volume 68". Editor: Hiroto S. Watanabe. Nova Science Publishers, Inc, Hauppauge NY, USA, pp. 125-151.

49. Schalow G (2002) Stroke recovery induced by coordination dynamic therapy and quantified by the coordination dynamic recording method. Electromyogr Clin Neurophysiol 42: 85-104.

50. Schalow G (2002) Improvement after traumatic brain injury achieved by coordination dynamic therapy. Electromyogr Clin Neurophysiol 42: 195-203.

51. Schalow G, Jaigma P (2006) Improvement in severe traumatic brain injury induced by coordination dynamics therapy in comparison to physiologic CNS development. Electromyogr Clin Neurophysiol 46: 195-209.

52. Schalow G, Jaigma P, Belle VK (2009) Near-total functional recovery achieved in partial spinal cord injury ( $50 \%$ injury) after 3 years of coordination dynamics therapy. Electromyogr Clin Neurophysiol 49: 67-91.

53. Schalow G (2003) Partial cure of spinal cord injury achieved by 6 to 13 months of coordination dynamic therapy. Electromyogr Clin Neurophysiol 43: 281-292. [Crossref]

54. Schalow G (2006) Cerebellar injury improvement achieved by coordination dynamics therapy. Electromyogr Clin Neurophysiol 46: 433-439.

55. Schalow G, Jaigma P (2005) Cerebral palsy improvement achieved by coordination dynamics therapy. Electromyogr Clin Neurophysiol 45: 433-445.

56. Schalow G (2006) Hypoxic brain injury improvement induced by coordination dynamics therapy in comparison to CNS development. Electromyogr Clin Neurophysiol 46: 171-183.

57. Schalow G, Pääsuke M, Ereline J, Gapeyeva H (2004) Improvement in Parkinson's disease patients achieved by coordination dynamics therapy. Electromyogr Clin Neurophysiol 44: 67-73.

58. Schalow G, Nyffeler T (2001) Koordinationsdynamik-Therapie: Myelomeningozele (Spina bifida). Physiotherapie.

59. Schalow G, Nyffeler T (2000) Koordinationsdynamik-Therapie: Skoliose Physiotherapie.

60. Schalow G (2018) Coma and spinal cord injury recovery achieved via coordination dynamics therapy. Clin. Case Rep. Rev. 4: 1-7.

61. Schalow G (2018) Paediatric acquired brain injury repair in 5 patients through Coordination Dynamics Therapy. Clin. Med. Rep 1: 1-19.

62. Koepchen HP (1990) Physiology of rhythms and control systems: An integrative approach. In: Haken H, Koepchen HP editors. Rhythms in Physiological Systems 3-20.

63. Lambertz M, Langhorst P (1998) Simultaneous changes of rhythmic organization in brainstem neurons, respiration, cardiovascular system and EEG between $0.05 \mathrm{~Hz}$ and 0.5 Hz. J. Auton Nerv Syst 68: 58-77.

64. Baehr M, Frotscher M (2005) Duus` Topical Diagnosis in Neurology. Thieme Verlag, Stuttgart. (a very good book for medical neuroscience because it correlates structure and function, only human neurophysiology is missing; old versions of Peter Duus are also good).

65. Schalow G (2006) Surface EMG- and coordination dynamics measurementsassisted cerebellar diagnosis in a patient with cerebellar injury. Electromyogr. Clin. Neurophysiol 46: 371-384.

66. Schalow G (2006). Symmetry diagnosis and treatment in coordination dynamics therapy. Electromyogr. Clin. Neurophysiol 46: 421-431. 
67. Schalow G, Zäch GA (2000) Reorganization of the Human CNS, Neurophysiologic measurements on the coordination dynamics of the lesioned human brain and spinal cord. Theory for modern neurorehabilitation (31 case reports). Gen. Physiol. Biophys 1: $1-244$.

68. Schalow G (2019) Permanent coma patient re-learned to speak via coordination dynamics therapy. Archives of Clinical and Medical Case Reports 3: 33-50.

69. Adolphs R (2002) Neural systems for recognizing emotion. Curr Opin Neurobiol 12: 169-177. [Crossref]

70. Alain, Pötter, Richard, Mazeron, Jean-Jacques et al. (2002) The GEC ESTRO handbook of brachytherapy. Leuven, Belgium: European Society for Therapeutic Radiology and Oncology.

71. Gasser HS, Grundfest H (1939) Axon diameter in relation to the spike dimension and the conduction velocity in mammalian A fibres. Amer. J. Physiol 127: 393-414.

72. Hursh JB (1939) Conduction velocity and diameter of nerve fibres. Amer. J. Physiol 127: $131-139$

73. Schalow G, Zäch GA (1994) Nerve compound action potentials analysed with the simultaneously measured single fibre action potentials. Electromyogr. Clin. Neurophysiol 34: 451-465.

74. Schalow G (2010) Phase relation changes between the firings of ? and ?-motoneurons and muscle spindle afferents in the sacral micturition centre during continence functions in brain-dead human and patients with spinal cord injury. Electromyogr. Clin. Neurophysiol 50: 3-27.
75. Schnakers C (2009) Diagnostic accuracy of the vegetative and minimally conscious state: clinical consensus versus standardized neurobehavioral assessment. BMC neurology 9: 35 .

76. Miledi R, Parker I, Schalow G (1977) Calcium entry across the postjunctional membrane during transmitter action. J. Physiol. 268: 32-33.

77. Miledi R., Parker I, Schalow G (1977) Calcium transients in frog slow muscle fibres, in frog skeletal muscle fibres using arsenazo III. Nature 5622: 750-752.

78. Deisseroth K, Singla S, Toda H (2004). Excitation-neurogenesis coupling in adult neural stem/progenitor cells. Neuron 42: 535-552.

79. Schalow G (2009) Building of new motoneurons in the human spinal cord upon coordination dynamics therapy to improve finger functions in motoric complete cervical spinal cord injury. In: Berkovsky, T.C. (Ed.), Handbook of Spinal Cord Injuries, Chapter 4. pp. 231-264, Nova Science Publishers.

80. Colvis CM, Pollock JD, Goodman RH, Impey S, Dunn J, et al. (2005) Epigenetic mechanisms and gene networks in the nervous system. $J$ Neurosci 25: 10379-10389. [Crossref]

81. Covic M, Karaca E, Lie DC (2010) Epigenetic regulation of neurogenesis in the adult hippocampus. Heredity (Edinb) 105: 122-134. [Crossref]

Copyright: (2019 Schalow G. This is an open-access article distributed under the terms of the Creative Commons Attribution License, which permits unrestricted use, distribution, and reproduction in any medium, provided the original author and source are credited. 